THE ROLE OF REX IN REGULATION

OF SULFATE REDUCTION IN

DESULFOVIBRIO VULGARIS HILDENBOROUGH

A Dissertation
presented to
the Faculty of the Graduate School
at the University of Missouri-Columbia
In Partial Fulfillment
of the Requirements for the Degree
Doctor of Philosophy
Dr. Judy D. Wall, Dissertation Supervisor
DECEMBER, 2014
GEOFFEY ALEX CHRISTENSEN


(C) Copyright by Geoffrey Alex Christensen 2014

All Rights Reserved 
The undersigned, appointed by the dean of the Graduate School, have examined the dissertation entitled

\title{
THE ROLE OF REX IN REGULATION OF SULFATE REDUCTION IN DESULFOVIBRIO VULGARIS HILDENBOROUGH
}

\author{
presented by Geoffrey Alex Christensen, \\ a candidate for the degree of Doctor of Philosophy, \\ and hereby certify that, in their opinion, it is worthy of acceptance.
}

Professor Judy D. Wall

Associate Professor Michael Calcutt

Professor Mark McIntosh

Associate Professor Brenda A. Peculis

Professor Frank J. Schmidt 
To my wife,

\section{Christina Christensen}

"May we grow old and grey together... just not too soon." 


\section{ACKNOWLEDGEMENTS}

In my youth I participated in many physically demanding activities including Hurdles in Track and Field, Cross Country and Epic Mud runs. The goal was always simple, make it to the end, improve every time, do not get hurt, and have fun. I approached earning my Doctorate in Biochemistry in a similar manner. That is to say, I knew that the next several years would be physically, as well as mentally, challenging for me and that I would need support along the way.

Therefore, I would like to first and foremost thank my supervisor, my coach, Dr. Judy D. Wall. Her continued support and guidance has developed me into the scientist I am today. With her testing of my limits I was able overcome substantial obstacles and achieve more than I could have ever imagined alone. Additionally, I wish to thank Judy's second-incommand, my assistant coach, Dr. Barbara Giles for making sure that I had the necessary skills and training to be successful in a laboratory environment.

I would also like to thank my committee members, my trainers and agents: Dr. Michael Calcutt, Dr. Mark McIntosh, Dr. Brenda Peculis and Dr. Frank Schmidt for their stimulating conversations during each of our meetings, making sure that I was not limiting myself to just one method or thought-process.

I am particularly grateful for the assistance given by Grant Zane, my personal trainer, for helping me develop the skills necessary to design and execute experiments, as well as to effectively communicate my work with others. Of course, no laboratory is a single person, and to that end I would like to thank my team, all those laboratory members past and 
present who directly or indirectly influenced the work that I am presenting in my thesis. Additionally, I would like to thank my collaborators, Dr. Alexey Kazakov, Dr. Dmitry Rodionov, Dr. Pavel Novichkov and Dr. Christopher Petzold for assisting in conducting experiments that were critical for my work.

My special thanks are extended to my sponsors for financially supporting me; Department of Biochemistry at University of Missouri, Ecosystems and Networks Integrated with Genes and Molecular Assemblies (ENIGMA), National Institute of General Medical Sciences (NIGMS) Training Grant, Dr. Charles W. Gehrke Jr. Memorial Scholarship Fund, American Society for Microbiology, and Federation of European Microbiological Societies (FEMS) Young Scientist Grant.

I would also like to express my very great appreciation to those who supported me on a more personal level, my family, my fans, especially my mother Lynn Guasta and Father Richard Christensen with his wife Karin. I also want to thank my grandparents for encouraging me, even though they may not have known exactly what I was doing, but that I enjoyed it. I wish to acknowledge the support of my in-laws, Mark and Cheryl Pope, for their support of both Christina and myself over the years.

And of course, I would like to thank my understanding wife, Christina Christensen, for her continued support over the past decade, especially prior to enrolling in graduate school. Having her by my side along this part of the race has made victory only that much more gratifying. I now stand at the end of this race victorious and capable of conquering the next big challenge, all thanks to my many supporters. 


\section{TABLE OF CONTENTS}

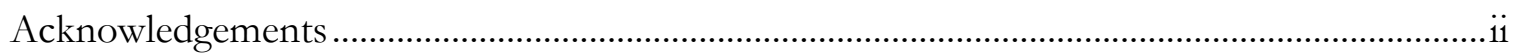

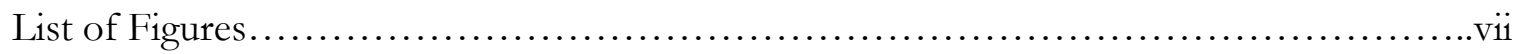

List of Tables.............................................................iii

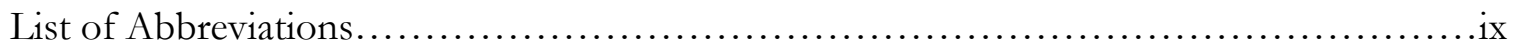

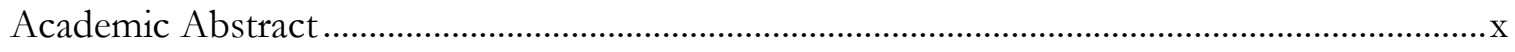

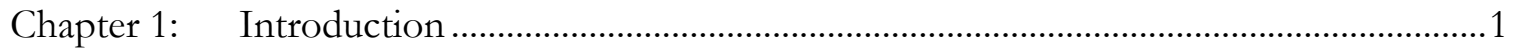

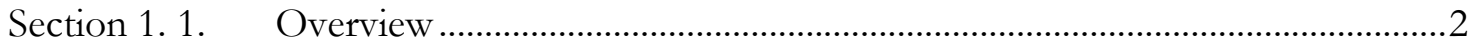

Section 1. 2. Microorganisms capable of reducing sulfate ..................................................2

Section 1. 3. Dissimilatory sulfate reduction ................................................................

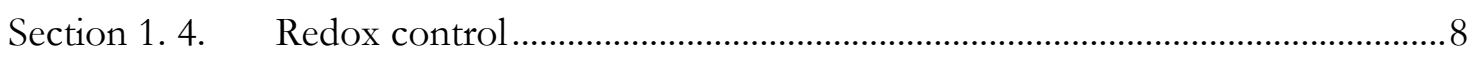

Section 1. 5. Regulation of sulfate reduction in SRM.....................................................

Section 1. 6. Gene transcription regulation in bacteria ........................................................

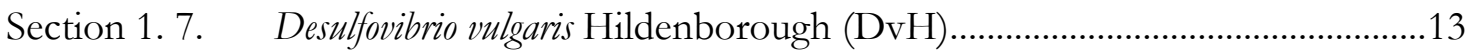

Section 1. 8. The transcriptional redox regulator Rex.........................................................14

Section 1. 9. Regulation of sulfate reduction genes by $\operatorname{Rex}_{\mathrm{DvH}}$........................................19

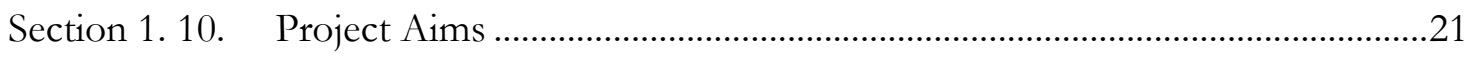

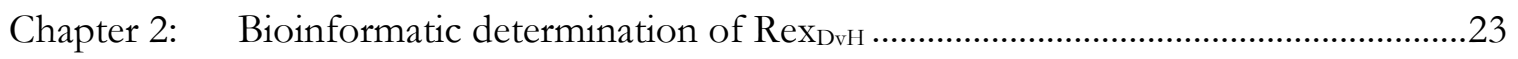

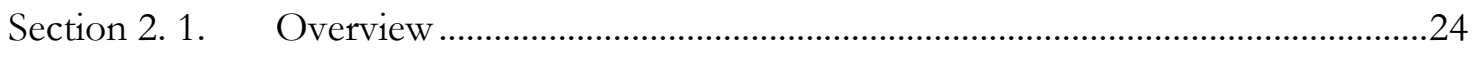

Section 2. 2. Sequence comparison..................................................................................24

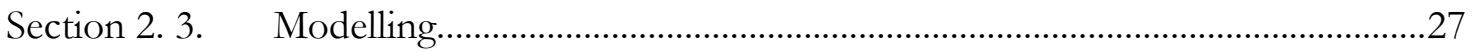

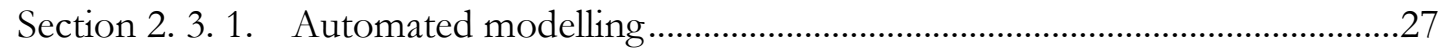

Section 2. 3. 2. Aligned modelling ....................................................................................... 30

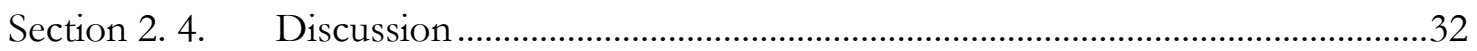

Chapter 3: $\quad \operatorname{Rex}_{\mathrm{DvH}}$ is a repressor of sat and its activity is modulated by $\mathrm{NADH}$..................34

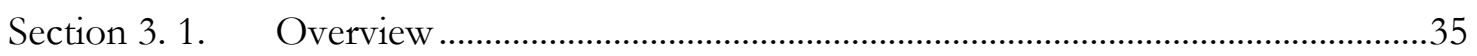

Section 3. 2. rex appears to be monocistronic ..................................................................... 35

Section 3. 3. Deletion of rex increased sat expression .........................................................37

Section 3. 4. Rex $\mathrm{x}_{\mathrm{DvH}}$ binds to the Rex $\mathrm{x}_{\mathrm{DvH}}$-binding site upstream of sat ............................39

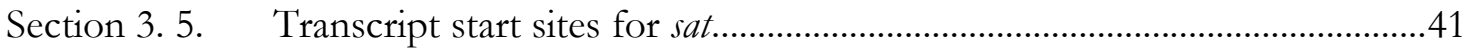

Section 3. 6. Effect of NADH concentrations on $\operatorname{ex}_{\text {DvH }}$ function ..................................43

Section 3. 7. Essential nucleotides of the Rex-binding motif ..........................................45 


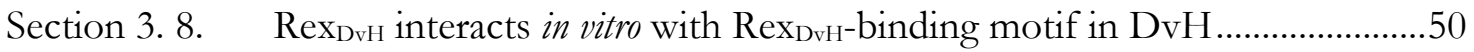

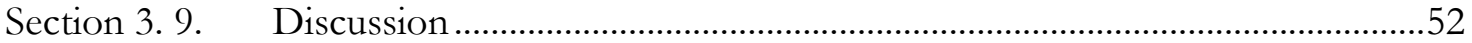

Chapter 4: Rex $\operatorname{DvH}_{\mathrm{Dv}}$ alters the electron flow between internal and external reduction

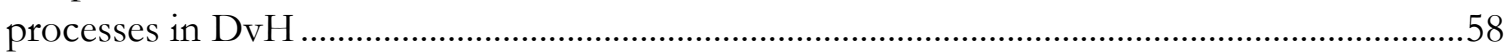

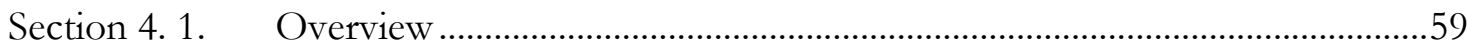

Section 4. 2. Whole-genome gene fitness of a Rex $x_{\mathrm{DvH}}$ mutant grown by lactate with sulfate respiration

Section 4. 3. The Rex mutant is impaired for growth with thiosulfate as the sole

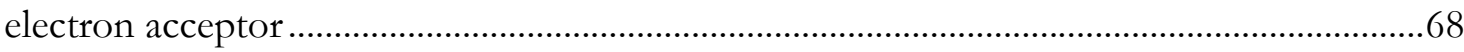

Section 4. 4. PhsA is not the only thiosulfate reductase in $\mathrm{DvH}$...................................73

Section 4. 5. Rex controls, directly or indirectly, the expression of $s a t$, aps $A$ and $d s r C .75$

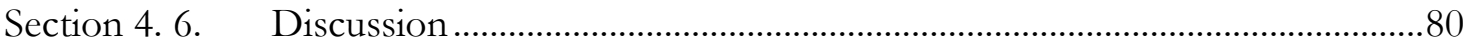

Chapter 5: Supplementary projects and discussion ................................................................98

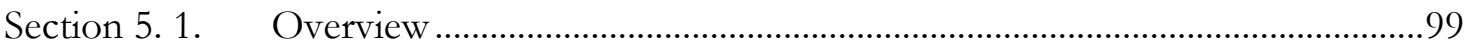

Section 5. 2. Yeast extract required for $\mathrm{DvH}$ to ferment pyruvate efficiently................99

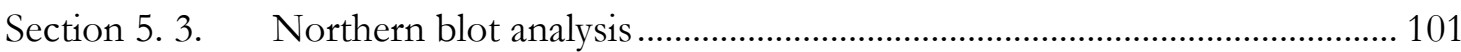

Section 5. 4. Rex mutant is sensitive to molybdate......................................................... 102

Section 5. 5. Other regulators predicted to control sulfate reduction ............................ 103

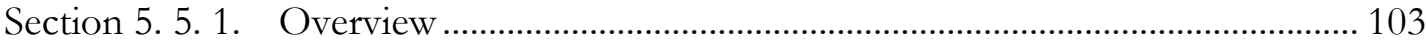

Section 5. 5. 2. Transcript expression results for potential regulators ........................... 104

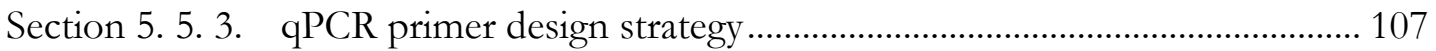

Section 5. 6. Additional RACE protocol and discussion.............................................. 109

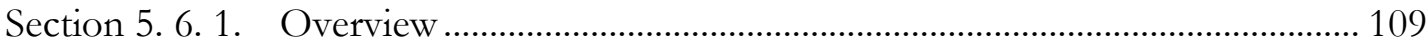

Section 5. 6. 2. Modifications to the TSS protocol ................................................................ 109

Section 5. 7. NADH quantification discussion ................................................................ 112

Chapter 6: General discussion and concluding remarks ...................................................... 115

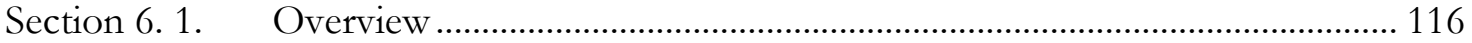

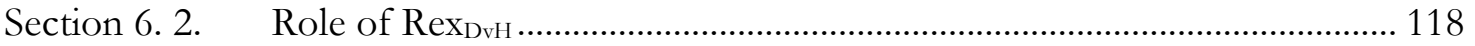

Section 6. 3. Future plans... where to next? .................................................................... 122

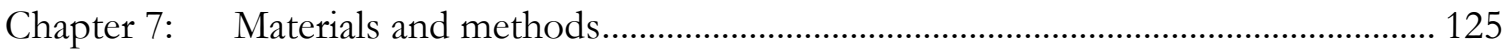

Section 7.1. Protein sequence modelling...................................................................... 126

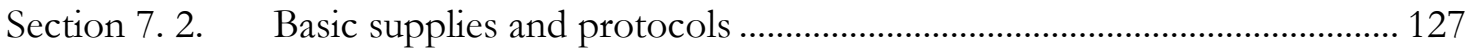

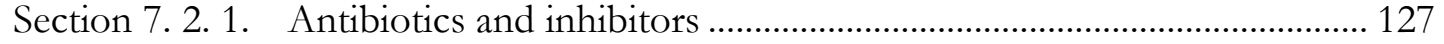




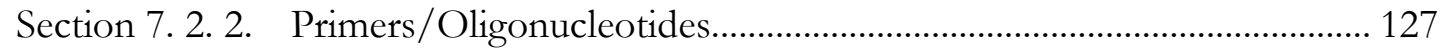

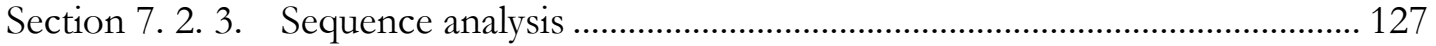

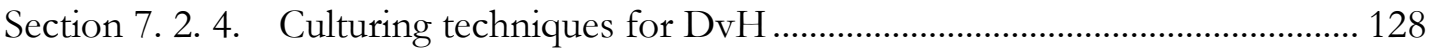

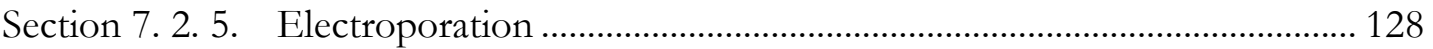

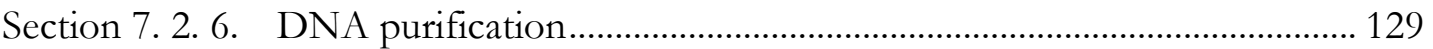

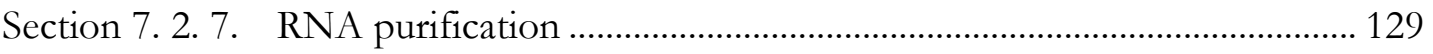

Section 7. 2. 8. Polymerase Chain Reaction (PCR) ........................................................ 130

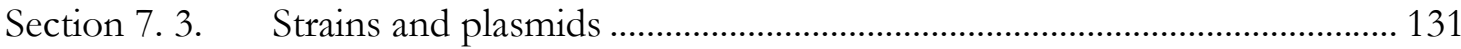

Section 7. 3. 1. Marker-exchange deletion of rex................................................................ 134

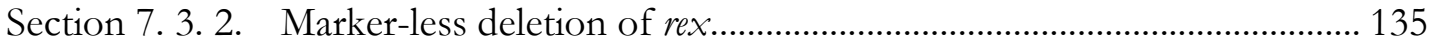

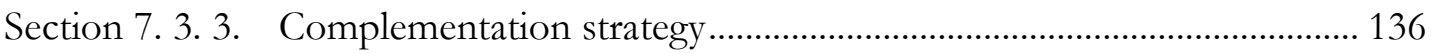

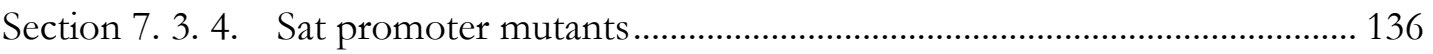

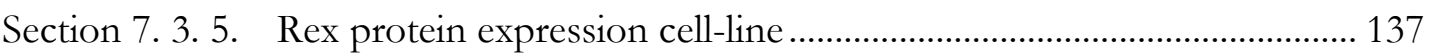

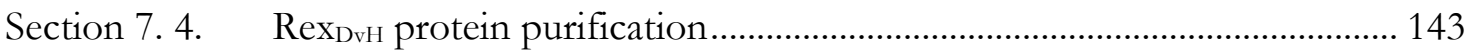

Section 7. 5. Rex operon determination by RT-PCR .................................................... 146

Section 7. 6. mRNA expression quantification by qRT-PCR …..................................... 147

Section 7. 6. 1. Primer design, protocol and analysis........................................................ 147

Section 7. 6. 2. Reference gene determination................................................................. 149

Section 7. 6. 3. Sample preparation for transcript analysis ............................................ 149

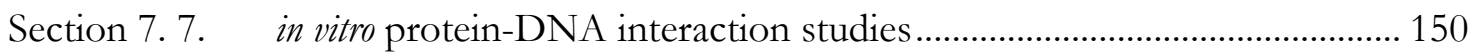

Section 7. 7. 1. Electrophoretic Mobility Shift Assay (EMSA) ..................................... 150

Section 7. 7. 2. Fluorescent Polarization Assay (FPA) ………....................................... 152

Section 7. 8. Select protein abundance determination .................................................... 155

Section 7. 9. Transcription start site (TSS) of sat determined by 5'-RACE................... 156

Section 7. 10. Whole-genome fitness study by TnLE-seq ................................................... 159

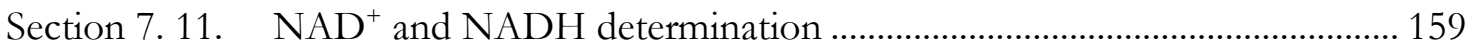

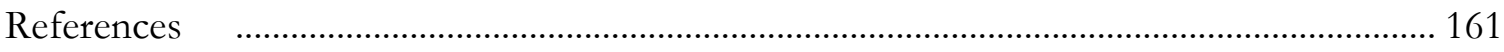

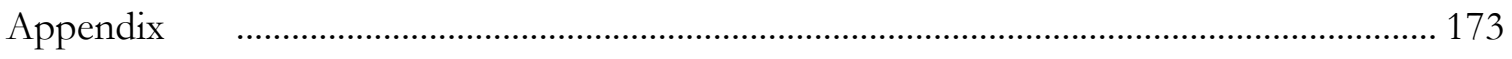

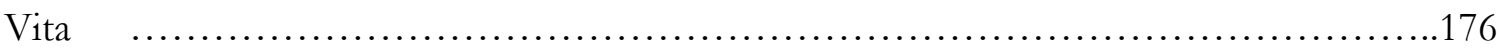




\section{LIST OF FIGURES}

Figure 1-1: Simplified schematic of sulfate reduction.................................

Figure 1-2: Main energy metabolism proteins in SRM.................................5

Figure 1-3: RNA polymerase holoenzyme $\left(\beta \beta^{\prime} \alpha_{2} \omega \sigma\right)$ positioning at a promoter................10

Figure 1-4: Scanning Electron Microscopy (SEM) of a single DvH cell......................13

Figure 1-5: Crystal structure of Rex bound to DNA from T. thermophiles HB27 .................16

Figure 1-6: Sequence logo of the Rex DNA-binding site in Desulfovibrionales...................16

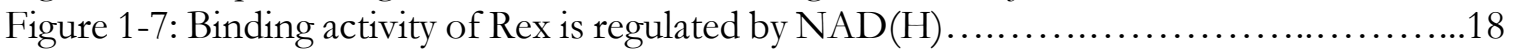

Figure 1-8: Simplified schematic of Rex conformational switch..........................18

Figure 2-1: Protein sequence alignment of Rex proteins.............................. 25

Figure 2-2: Sequence alignment of Rex homologs throughout Desulfovibrionales................26

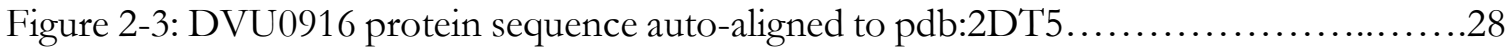

Figure 2-4: DVU0916 protein sequence aligned to Rex from $T$. aquaticus.................... 30

Figure 3-1: RT-PCR confirms rex to be monocistronic...................................

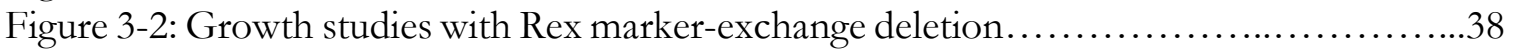

Figure 3-3: $\operatorname{Re}_{\mathrm{DvH}}$ interacts in vitro with sat promoter, shown by EMSA ...................40

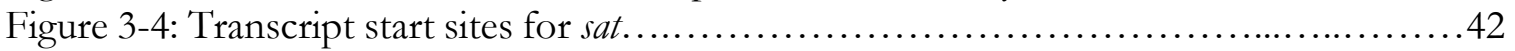

Figure 3-5: NADH disrupts interaction between $\operatorname{Rex}_{\mathrm{DvH}}$ and the $\operatorname{Rex}_{\mathrm{DvH}}-$ binding site.........44

Figure 3-6: Growth kinetics of parental and sat promoter mutants......................47

Figure 3-7: Electrophoretic assay demonstrating Rex $\mathrm{D}_{\mathrm{DvH}}$ binding to consensus site...........49

Figure 4-1: Minimal difference in gene fitness between the Rex mutant and parental strain....61

Figure 4-2: Minimal difference in gene fitness....................................62

Figure 4-3: Deletion of rex, and to an extent $p h s A$, impair growth in thiosulfate..............69

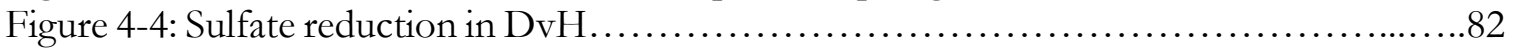

Figure 5-1: Pyruvate fermentation control experiment..............................100

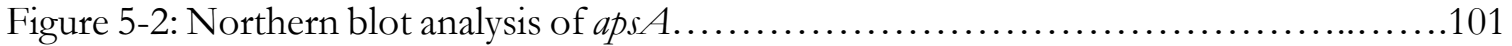

Figure 5-3: Rex mutant is more sensitive to molybdate than the parental strain..............102

Figure 5-4: Primer optimization protocol of a "good" and "bad" primer set..................108

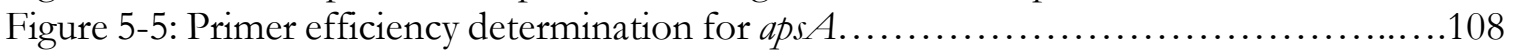

Figure 5-6: Pyridine nucleotide determination...................................... 113

Figure 5-7: Media components disrupt pyridine nucleotide assay..................... 114

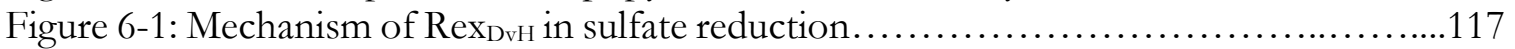

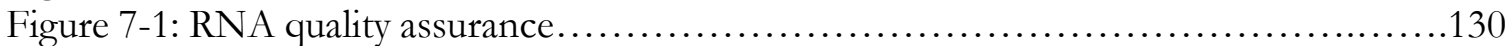

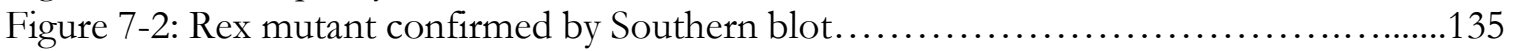

Figure 7-3: Construction of pMO3312 (Rex expression plasmid) ........................138

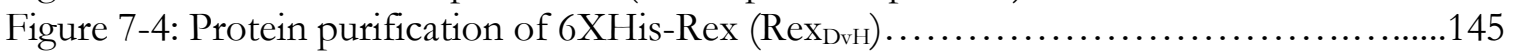

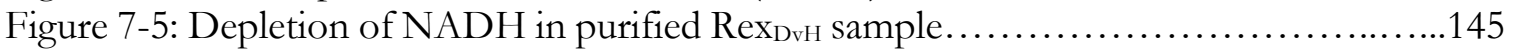

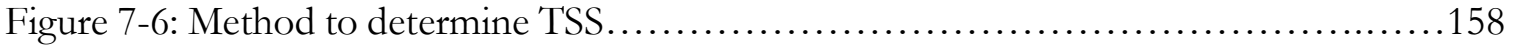




\section{LIST OF TABLES}

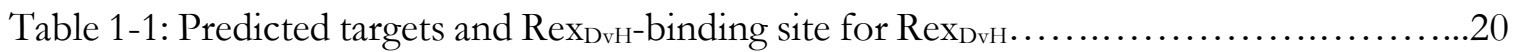

Table 2-1: Protein sequence identity among Rex homologs............................. 24

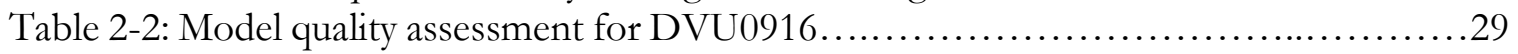

Table 3-1: Growth and transcript analysis of Rex marker-exchange deletion...................39

Table 3-2: Alteration to the Rex $\mathrm{x}_{\mathrm{DvH}}$-binding site within the sat promoter...................46

Table 3-3: Transcript analysis of parental and $\operatorname{Rex}_{\mathrm{DvH}^{-}}$-binding site alteration strains..........48

Table 3-4: Rex $x_{\text {DvH }}$ interaction with predicted $\operatorname{Rex}_{\mathrm{DvH}}$-binding sites......................51

Table 4-1: Gene fitness for parental strain and Rex mutant grown by sulfate respiration......63

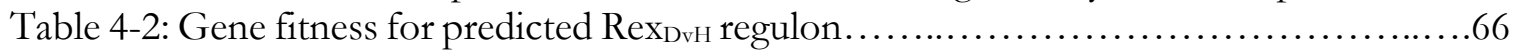

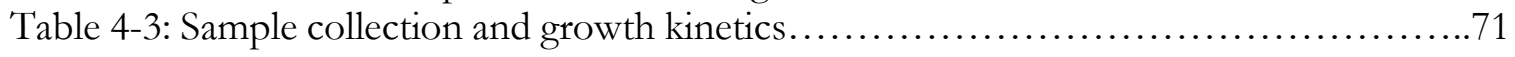

Table 4-4: Transcript analysis of Rex mutant in several different media.................. 75

Table 4-5: Transcript analysis of Rex mutant and parental strain ........................76

Table 4-6: Transcript analysis of parental strain, JW710, in several different media...........77

Table 4-7: Protein analysis of parental strain and Rex mutant in several different.............79

Table 4-8: DvH Transcript analysis as determined by Microarray........................ 85

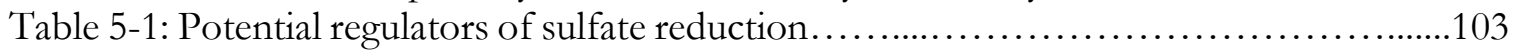

Table 5-2: Sample collection and transcript analysis of potential regulators.................105

Table 5-3: Transcript analysis of potential regulators ...................................

Table 5-4: Primer list for RACE experiments (cloning method) ........................111

Table 7-1: Strains.............................................................. 132

Table 7-2: Plasmids.......................................................... 133

Table 7-3: Primer list for strains and plasmids generation..............................139

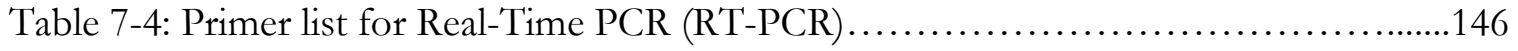

Table 7-5: Primer list for quantitative Real-Time PCR (qRT-PCR) . . . . . . . . . . . . . . . . ...148

Table 7-6: Primer list for Electrophoretic Mobility Shift Assays (EMSA) ...................151

Table 7-7: Primer list for Fluorescent Polarization Assay (FPA) ........................153

Table 7-8: Primer list for 5'-Rapid Amplification of cDNA Ends (5'-RACE) ................157

Table A-1: Additional transcript data for Rex MED ................................ 174

Table A-2: Sample collection and transcript analysis for sat promoter studies................175 


\section{LIST OF ABBREVIATIONS}

\begin{tabular}{|c|c|}
\hline $5-\mathrm{FU}$ & 5-fluorouracil \\
\hline $\mathrm{Aa}$ & Amino acid \\
\hline Ap & Ampicillin \\
\hline bp & Base pairs \\
\hline cDNA & copy DNA \\
\hline $\mathrm{Cq}($ or $\mathrm{Ct})$ & Critical Threshold (qPCR) \\
\hline DMSO & Dimethyl sulfoxide \\
\hline DNA & Deoxyribonucleic acid \\
\hline $\mathrm{DvH}$ & Desulfovibrio vulgaris Hildenborough \\
\hline EMSA & Electrophoretic mobility shift assay \\
\hline FPA & Fluorescent polarization assay \\
\hline G418 & Geneticin $\mathbb{R}$ \\
\hline gDNA & Genomic DNA \\
\hline $\mathrm{Km}$ & Kanamycin \\
\hline MED & Marker-exchange deletion \\
\hline MLD & Marker-less deletion \\
\hline $\mathrm{NAD}^{+}$ & Nicotinamide adenine dinucleotide (oxidized form) \\
\hline $\mathrm{NADH}$ & Nicotinamide adenine dinucleotide (reduced form) \\
\hline NTC & No Template Control \\
\hline $\mathrm{pdb}$ & Protein data bank \\
\hline $\operatorname{Rex}_{\mathrm{DvH}}$ & Rex protein homolog in $\mathrm{DvH}$ \\
\hline RFU & Relative fluorescence units \\
\hline RNA & Ribonucleic acid \\
\hline RNAP & RNA polymerase \\
\hline Sp & Spectinomycin \\
\hline SRB & Sulfate-reducing bacteria \\
\hline SRM & Sulfate-reducing microbe \\
\hline $\mathrm{T}_{\mathrm{m}}$ & Melting Temperature \\
\hline TSS & Transcript start site \\
\hline \multicolumn{2}{|c|}{ Media components: } \\
\hline \multicolumn{2}{|c|}{ MO: $\quad$ basal salt (standard medium) } \\
\hline \multicolumn{2}{|c|}{ L: $\quad$ lactate $(60 \mathrm{mM})$} \\
\hline \multicolumn{2}{|c|}{ P: $\quad$ pyruvate $(60 \mathrm{mM})$} \\
\hline \multicolumn{2}{|c|}{ S4: $\quad$ sulfate, $\mathrm{SO}_{4}^{2-}(30 \mathrm{mM})$} \\
\hline \multicolumn{2}{|c|}{ S3: $\quad$ sulfite, $\mathrm{SO}_{3}^{2-}(40 \mathrm{mM})$} \\
\hline \multicolumn{2}{|c|}{ T3: thiosulfate $\mathrm{S}_{2} \mathrm{O}_{3}{ }^{2-}(30 \mathrm{mM})$} \\
\hline \multicolumn{2}{|r|}{ pyruvate fermentation mediun } \\
\hline Y: & yeast extract \\
\hline
\end{tabular}




\title{
THE ROLE OF REX IN REGULATION OF SULFATE REDUCTION IN DESULFOVIBRIO VULGARIS HILDENBOROUGH
}

\author{
Geoffrey Alex Christensen
}

\author{
Dr. Judy Wall, Dissertation Supervisor
}

\begin{abstract}
Although the enzymes for dissimilatory sulfate reduction by microbes have been studied, the mechanism for transcriptional regulation of the encoding genes remain unknown. In the work presented here, the model sulfate-reducing microbe Desulfovibrio vulgaris Hildenborough $(\mathrm{DvH})$ was used to determine the role of Rex in sulfate reduction regulation. A deletion of the putative rex gene was made in DvH. the Rex mutant was assayed for growth with different combinations of electron donors and acceptors. Growth of the Rex mutant was less efficient on thiosulfate-containing medium. Here we propose a new model for thiosulfate reduction in SRB and examine a putative thiosulfate reductase. Additionally, transcript expression studies focused on sat, encoding sulfate adenylyl transferase, showed increased levels in the Rex mutant relative to the parental strain confirming Rex to be a repressor of sat. The putative Rex-binding site upstream of sat was also confirmed. We established in vitro that the presence of elevated NADH disrupted the interaction between Rex and DNA. these data support the role of Rex as a transcription repressor for sat that senses the redox status of the cell through $\mathrm{NADH} / \mathrm{NAD}^{+}$.
\end{abstract}


Chapter 1: $\quad$ Introduction 


\section{Section 1. 1. Overview}

Sulfate-reducing microbes (SRM) are challenged with a range of environmental stresses, such as nutrient availability or $\mathrm{O}_{2}$ levels, and therefore need to be able to respond to these changes. One potential mechanism would be through a sensory regulatory pathway that can switch between growth modes (e.g. respiration and fermentation). Such a change in growth mode may be detected by a sensory protein that transduces the signal to alter gene expression and, subsequently, alter the functional activity of the cell. Many levels of regulation are involved in the complex process of energy conversion. This study focused on characterizing the recently annotated redox regulator, Rex, in the model anaerobic SRM Desulfovibrio vulgaris Hildenborough (DvH). The protein Rex senses changes in the $\mathrm{NADH} / \mathrm{NAD}^{+}$ratio in response to limitations in oxidative phosphorylation processes, and it controls the genes that code for the proteins responsible for these processes. Therefore, $\operatorname{Rex}_{\mathrm{DvH}}$ is predicted to control sulfate reduction in SRM. This chapter focuses on the role of SRM in the environment, on gene regulation and on the putative regulator Rex.

\section{Section 1.2. Microorganisms capable of reducing sulfate}

Bacteria have developed the capabilities to couple reductive processes to energy conversion through oxidative phosphorylation, with each substrate used providing a specific amount of energy. Examples of reductive processes include the following: $\mathrm{CO}_{2}$ reduction to methane, sulfate reduction to sulfide, fumarate reduction to succinate, nitrate reduction to nitrite, nitrite reduction to $\mathrm{N}_{2}$, and $\mathrm{O}_{2}$ reduction to $\mathrm{H}_{2} \mathrm{O}$ (Thauer et al. 1977). The energy gained from the anaerobic reduction of sulfate to sulfide $\left(\Delta G^{o t}=-18.8 \mathrm{~kJ} / \mathrm{mol}\right)$ is low as compared to the reduction of other substrates, for example, nitrite reduction to nitrogen $\left(\Delta \mathrm{G}^{\mathrm{ol}}=\right.$ $132.6 \mathrm{~kJ} / \mathrm{mol}$ ) (Thauer et al. 1977). SRM are anaerobic microorganisms (archaea and 
bacteria) that are nearly ubiquitous in anoxic environments and use sulfate as a terminal electron acceptor for the degradation of organic compounds and energy conversion (Muyzer et al. 2008). SRM were first reported in 1895 (Beijerinck 1895); however, it was not until much later that their importance was realized. Sulfate reducers have been separated into two different groups based on their ability to oxidize organic compounds completely to carbon dioxide or incompletely to acetate (Muyzer et al. 2008). In either case, sulfate is dissimilated, or used for energy conversion and growth. This process is separate from the reduction of sulfate for assimilation of sulfur into macromolecules such as proteins. SRM are abundant in nature and considered to be important microbes in anaerobic carbon and sulfur cycles (Pfennig et al. 1982) and account for about half of the degradation of organic material in anoxic environments (Jorgensen 1977)

Although SRM are primarily found in sulfate-rich anoxic environments (Cypionka 2000), they are also abundant in habitats depleted of sulfate because they can use electron acceptors other than sulfate (e.g. sulfite or thiosulfate) (Thauer et al. 2007; Muyzer et al. 2008). The environments are as diverse as the substrates that they can utilize, such as sugars (Sass et al. 2002), amino acids (Baena et al. 1998) and one-carbon compounds (e.g. carbon monoxide (Parshina et al. 2005)). These microbes are associated with wetlands, wastewater treatment, environmental nutrient cycles, food spoilage, geochemical transformation, fuel production, animals, bioremediation and biocorrosion (Barton 1995).

It has been estimated that the annual cost of corrosion world-wide is $\$ 2.2$ trillion and is approximately 3\% of the world's Gross Domestic Product (GDP) (Hays 2010). In 2001, corrosion was estimated to cost the U.S. more than $\$ 600$ billion, an equivalent of each citizen having to pay $\$ 2000$ each year (Bushman 2001). There have been advances made for corrosion control, including material selection, coatings, inhibitors, and cathodic protection 
(Davis 2000); however, these all have their costs as well. The anaerobic process of microbially influenced corrosion is estimated to account for $15 \%$ of the total cost of ferrous metal and concrete/stonework corrosion (Enning et al. 2013). For the U.S. energy industry, this amounts to approximately $\$ 100$ billion yearly (Enning et al. 2013). Most notably, the metabolic processes of the SRM have been implicated in this observed corrosion and are, therefore, meaningful to study (Lee et al. 1995; Hamilton 2003).

\section{Section 1.3. Dissimilatory sulfate reduction}

The process of dissimilatory sulfate reduction is carried out by a well-conserved biochemical pathway (Figure 1-1), which is part of a larger energy metabolism pathway of SRM (Error!

Reference source not found.) (Peck et al. 1994; Barton et al. 2007; Thauer et al. 2007; Dahl et al. 2008; Muyzer et al. 2008; Pereira et al. 2011).

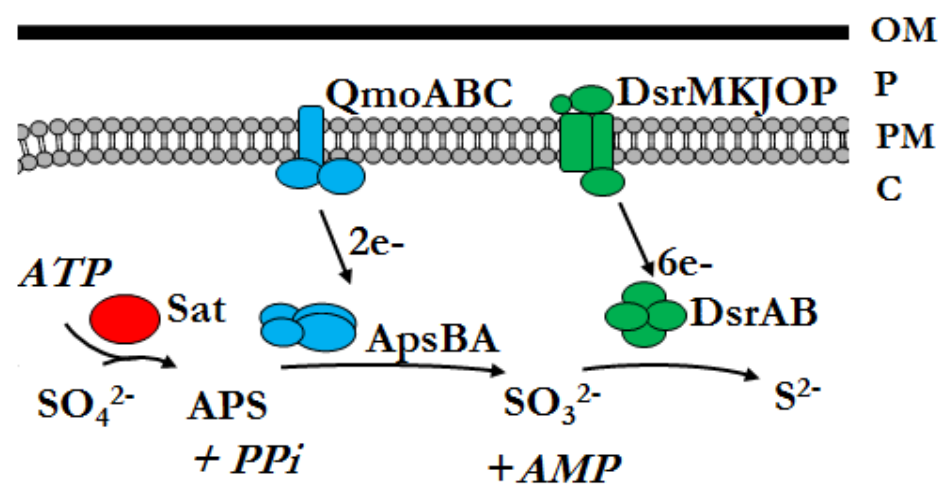

Figure 1-1: Simplified schematic of sulfate reduction. Sat, sulfate adenylyl transferase; ApsBA, APS reductase; DsrAB, dissimilatory sulfite reductase; QmoABC, quinone-interacting membrane bound oxidoreductase; DsrMKJOP, membrane portion of dissimilatory sulfite reductase; $\mathrm{SO}_{4}{ }^{2-}$, sulfate; APS, adenosine phosphosulfate; $\mathrm{SO}_{3}{ }^{2-}$, sulfite; $\mathrm{S}^{2-}$, sulfide; OM, outer membrane, P, periplasm; PM, periplasmic membrane; C, cytoplasm 


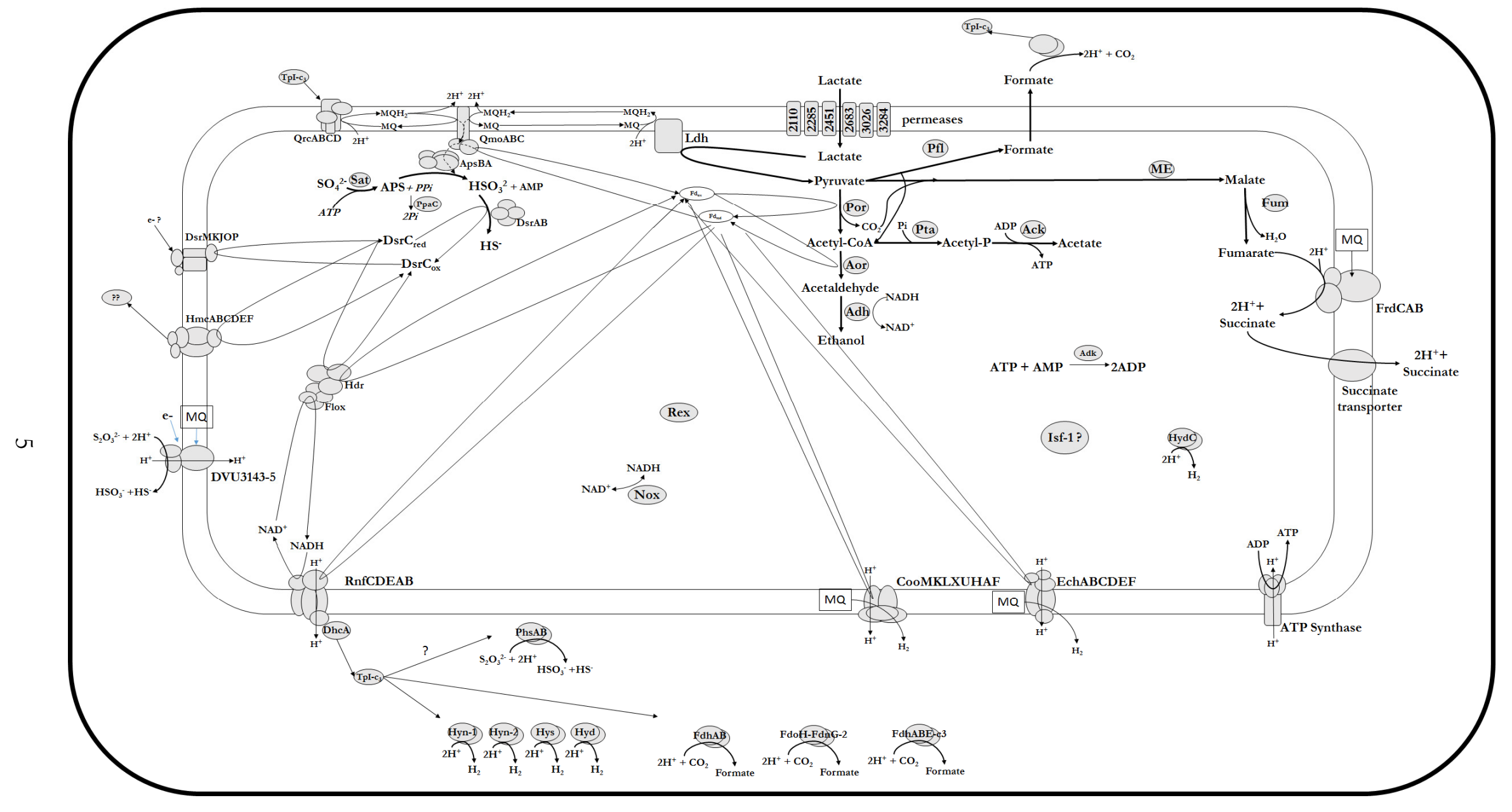




\section{Figure 1-2: Main energy metabolism proteins in SRM.}

Cytochrome c3 (TpI-c3), quinone reductase (QrcABCD), quinoneinteracting membrane-bound oxidoreductase (QmoABC), APS reductase (ApsBA), sulfate adenylyl transferase (Sat), pyrophosphatase $(\mathrm{PpaC})$, adenylate kinase (Adk), dissimilatory sulfite reductase (DsrAB, DsrMKJOP and DsrC), lactate dehydrogenase (Ldh), Lactate permeases (DVU2110, DVU2285, DVU2451, DVU2683, DVU3026, DVU3284), pyruvate oxidoreductase (Por), aldehyde:ferredoxin oxidorectase (Aor), alcohol dehydrogenase (Adh), phosphate acetyltransferase (Pta), acetate kinase (Ack), pyruvate:formate lyase (Pfl), heterodisulfide reductase (Hdr-Flox), decaheme cytochrome c (DhcA) NADH:quinone oxidoreductsae (RnfCDEAB), thiosulfate reductase (PhsAB), cytoplasmic hydrogenases (Hyn-1, Hyn-2, Hys, Hyd), periplasmic hydrogenases (CooMKLXUHAF, EchABCDEF), formate dehydrogenases (FdhAB, FdoH-FdnG-2, FdhABE-c3), high molecular weight cytochrome (HmcABCDEF), malate enzyme (ME), Fumarate reductase (FrdCAB), NADH:oxidoreductase (Nox), sulfate $\left(\mathrm{SO}_{4}{ }^{2-}\right)$, adenylyl phosphosulfate (APS), sulfite $\left(\mathrm{HSO}_{3}{ }^{-}\right)$, sulfide $\left(\mathrm{HS}^{-}\right)$. 
The enzymatic equations and the free energy $\left(\Delta \mathrm{G}^{\mathrm{ol}}\right)$ associated with these processes are shown below (Thauer 1989):

$$
\begin{array}{ll}
\mathrm{SO}_{4}{ }^{2-}+\mathrm{ATP} \rightarrow \mathrm{APS}+\mathrm{PP}_{\mathrm{i}} & \Delta \mathrm{G}^{\mathrm{ol}}=+46.0 \mathrm{~kJ} / \mathrm{mol} \\
\mathrm{APS}+2[\mathrm{H}] \rightarrow \mathrm{SO}_{3}{ }^{2-}+2 \mathrm{H}^{+}+\mathrm{AMP} & \Delta \mathrm{G}^{\mathrm{ol}}=-66.7 \mathrm{~kJ} / \mathrm{mol} \\
\mathrm{PP}_{\mathrm{i}}+\mathrm{H}_{2} \mathrm{O} \rightarrow 2 \mathrm{P}_{\mathrm{i}} & \Delta \mathrm{G}^{\mathrm{ol}}=-21.9 \mathrm{~kJ} / \mathrm{mol} \\
\mathrm{AMP}+\mathrm{ATP} \rightarrow 2 \mathrm{ADP} & \Delta \mathrm{G}^{\mathrm{ol}}=
\end{array}
$$

In brief, sulfate $\left(\mathrm{SO}_{4}{ }^{2-}\right)$ is taken up by the cell through an active transport system and is activated by sulfate adenylyl transferase (Sat, encoded by sat) to adenosine phosphosulfate (APS) and inorganic pyrophosphate $\left(\mathrm{PP}_{\mathrm{i}}\right)$. APS is then reduced to sulfite $\left(\mathrm{SO}_{3}{ }^{2-}\right)$ and AMP by APS reductase (ApsBA, encoded by apsB A) as seen in equations [1] and [2], respectively. High specific activities of inorganic pyrophosphatase ( $\mathrm{PpaC}$, encoded by $p p a \mathrm{C})$ and adenylate kinase (Adk, encoded by adk), equations [3] and [4], respectively, allow for this process to proceed in the forward direction. The reduction of sulfate requires an input of energy, as indicated by a positive free energy in equation [1]. Two molecules of ATP are required for each sulfate reduced, as seen when equations [1] to [4] are combined to yield equation [5].

$$
\begin{array}{r}
\mathrm{SO}_{4}{ }^{2-}+2 \mathrm{ATP}+\mathrm{H}_{2} \rightarrow \mathrm{SO}_{3}{ }^{2-}+2 \mathrm{H}^{+}+2 \mathrm{ADP}+2 \mathrm{P}_{\mathrm{i}} \\
\Delta \mathrm{G}^{0^{\prime}}=-42.6 \mathrm{~kJ} / \mathrm{mol}
\end{array}
$$

As shown in equation [6], sulfite is further reduced to hydrogen sulfide $\left(\mathrm{H}_{2} \mathrm{~S}\right)$ by dissimilatory sulfite reductase (DsrAB, encoded by $d s r A B)$. This is an exergonic reaction with enough free energy to drive the synthesis of two to three molecules of ATP, and thus overcome the initial energy demands.

$$
\mathrm{SO}_{3}{ }^{2-}+3 \mathrm{H}_{2}+2 \mathrm{H}^{+} \rightarrow \mathrm{H}_{2} \mathrm{~S}+3 \mathrm{H}_{2} \mathrm{O} \quad \Delta \mathrm{G}^{\mathrm{o}^{\prime}}=-179 \mathrm{~kJ} / \mathrm{mol}
$$




\section{Section 1. 4. Redox control}

Significant alterations occur within a cell during the switch between respiration (i.e. oxidative phosphorylation) and fermentation (i.e. substrate-level phosphorylation) processes. Aerobes, when grown in the presence of oxygen, utilize many global regulators for controlling growth through changes in gene expression (Bauer et al. 1999). For example, transcription factors SoxR (Greenberg et al. 1990) or OxyR (Altuvia et al. 1994) regulate oxygen defense proteins, while other proteins (e.g. ArcB (Iuchi et al. 1988) and FNR (Sawers et al. 1988)) control the switch between growth modes (i.e. aerobic/respiration versus anaerobic/fermentation). Each of these "redox regulators" have specific sensitivities to oxygen and through these sensitivities provide for a fine-tuning mechanism to sense and respond to the presence (or absence) of oxygen or cellular redox state. Different mechanisms have evolved to sense the redox state of a cell, including iron-sulfur centers (e.g. SoxR or FNR), heme (e.g. FixL), flavin (e.g. NifL), disulfide bond formation (e.g. OxyR), and many others currently uncharacterized (e.g. RegB) (Bauer et al. 1999). More recently, pyridine nucleotides (specifically the ratio $\mathrm{NADH} / \mathrm{NAD}^{+}$), sensed by the transcriptional repressor Rex, have been shown to control for redox as well (Brekasis et al. 2003). In DvH, Rex has been identified (Ravcheev et al. 2012), although other proteins have also been examined for their role in sensing the environmental redox state including two methyl-accepting chemotaxis proteins (DcrA (Fu et al. 1994); DcrH (Xiong et al. 2000)). The validation of the role of pyridine nucleotides as a redox signal for Rex will be part of the work presented in this thesis. 


\section{Section 1. 5. Regulation of sulfate reduction in SRM}

The process of dissimilatory sulfate reduction has been studied extensively. Although the enzymes for sulfate reduction have been well studied (Peck et al. 1994; Thauer et al. 2007;

Dahl et al. 2008), and their crystal structures solved (Ullrich et al. 2001; Fritz et al. 2002;

Oliveira et al. 2008; Schiffer et al. 2008), the mechanism for transcriptional regulation of the genes that code for these proteins remains unknown. In numerous SRM over 100

transcriptional regulators have been annotated within their genomes, with many regulators predicted to have the potential to regulate sulfate reduction, including: Rex (Ravcheev et al. 2012), HcpR (Rodionov et al. 2004), LysX and other DNA-binding proteins several of which are hypothetical proteins (Turkarslan et al. 2013).

Examination of mRNA transcription levels for several SRM reveal that many genes that code for proteins responsible for sulfate reduction are differentially expressed depending on the available nutrients (www.microbesonline.org) (Dehal et al. 2010). For example, sat and aps $B A$, which code for enzymes responsible for reducing sulfate to sulfite, have been shown to be altered in expression depending on the available electron acceptor (Wall et al. 2008; Zane et al. 2010). The work presented here will focus on the regulation of genes that code for proteins responsible for sulfate reduction.

\section{Section 1. 6. Gene transcription regulation in bacteria}

Transcription in bacteria is due to the RNA polymerase (RNAP) (Figure 1-3). The core enzyme, composed of $\beta \beta^{\prime} \alpha_{2} \omega$ subunits, is capable of DNA-dependent RNA synthesis. However, transcription initiation at a specific promoter requires an additional subunit, $\sigma$. Therefore, the $\beta \beta^{\prime} \alpha_{2} \omega \sigma$ holoenzyme is required for transcription initiation (Burgess et al. 1969). The principal or housekeeping $\sigma$ factor is the $\sigma^{70}$ and is responsible for most 
transcription (Paget et al. 2003). It has been reported that on average there are several thousand RNAP core molecules, and greater than 1.3-fold more $\sigma^{70}$ per cell (Grigorova et al. 2006). The number of holenzymes per cell is in excess of the number of growing chains, and thus, there is a pool of polymerases not synthesizing RNA.

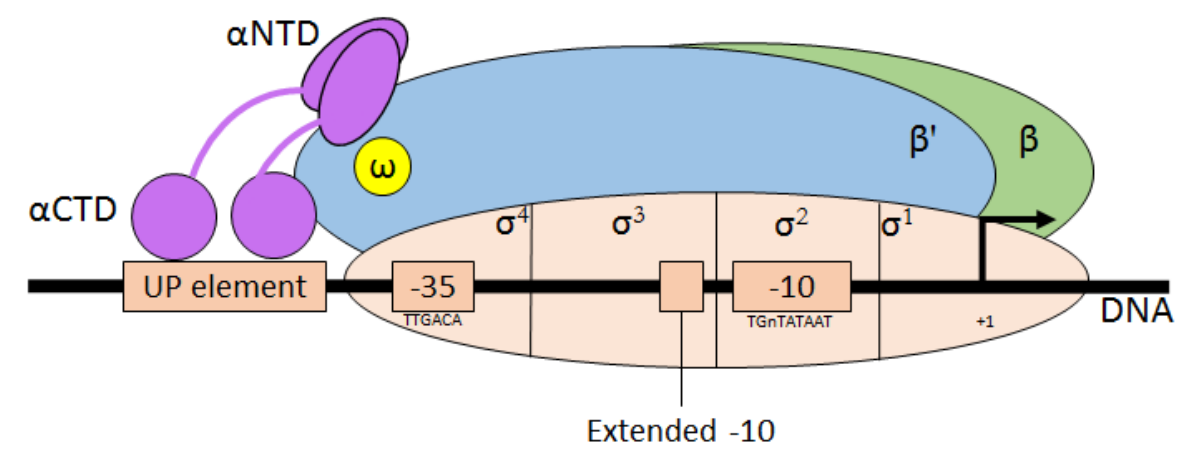

Figure 1-3: RNA polymerase holoenzyme $\left(\beta \beta^{\prime} \alpha_{2} \omega \sigma\right)$ positioning at a promoter. Transcript start site (TSS) marked by bent arrow. NTD, N-terminal domain; CTD, C-terminal domain. UP, upstream. Sigma subunit $(\sigma)$ shown as four domains $\left(\sigma^{4}, \sigma^{3}, \sigma^{2}, \sigma^{1}\right)$. Figure adapted from Lee et al. (2012).

Transcript initiation occurs in three steps. First, the $\sigma$ factor of the holoenzyme must recognize a promoter, although $\sigma$ may interact with DNA independent of the rest of the holoenzyme, and it does so through its interactions with elements located upstream of a transcript start site (TSS). Secondly, RNAP forms an open complex, which allows for the template strand to be inserted into the active site of RNAP. Thirdly, there is transcription of the complementary RNA strand (Murakami et al. 2003). The efficiency of transcription is dependent on each of these steps, with the rate-limiting step determining the overall expression level. Typically, the initial binding of the RNAP holoenzyme to the promoter or the transition to the open state is the rate-limiting step (Rojo 1999). 
The principal promoter elements recognized by RNAP include the UP (upstream) element, the -35 element, the extended -10 element, and the -10 element (Figure 1-3), although not all elements may be present for a given promoter. Each region is recognized by one of the four domains of the $\sigma$-subunit, and there is additional promoter recognition by the C-terminal domains of the two $\alpha$-subunits ( $\alpha$ CTDs) (Ebright et al. 1995; Gruber et al. 2003). An important component of the $\alpha$ subunit is the flexible unstructured linker between the $\mathrm{N}$ - and C-terminal domains that allows for regulation but has no role in the catalysis of RNA synthesis (Gourse et al. 2000). In most cases, the initial interaction of RNAP involves the UP and -35 elements with the downstream elements involved later.

Bacteria have developed numerous mechanisms to control gene expression ranging from initial promoter recognition through degradation of the protein. Genes can be turned on or off by alterations in RNAP, by DNA rearrangements, by a regulatory protein(s) or short RNA(s) affecting transcription initiation, or by modulation of the necessary components for initiation, elongation or termination (Rojo 1999). Additional regulation of the resulting transcript further controls the overall activity of a gene, including: mRNA and protein stability, translation efficiency, and protein turnover (Rojo 1999). A comprehensive list of bacterial transcriptional regulation is presented elsewhere (Lee et al. 2012).

Generally, repressors operate by binding to a specific promoter sequence in a way that would impede RNAP from binding or activating transcription. Multiple mechanisms for repressors have been observed. 1) Overlapping binding sites for the repressor and RNAP. For example, Rex binds to the $c y d_{\mathrm{p} 1}$ promoter (positions -6 to +26 ) and occludes binding of RNAP in Streptomyces coelicolor A3(2) (Brekasis et al. 2003). Another example is the wellstudied LacI repressor, which binds to operator $\mathrm{O}_{1}$ in the absence of lactose (Schlax et al. 1995). 2) Selection or availability of $\sigma$ factors to the core enzyme in the holoenzyme. An 
example of this is the anti- $\sigma$ factor, FlgM, which controls the accessibility of $\sigma^{28}$ during flagellum development in Salmonella enterica serovar typhimurium (Chadsey et al. 1998). 3) Repressor binding site is located outside of RNAP binding. For example, CytR is a repressor that prevents the activation of $\operatorname{de} C A B D$ by blocking CRP activation in Escherichia coli (Valentin-Hansen et al. 1996). 4) Mechanisms to control gene expression through other means may include: blocking the transition for the closed to open complex or inhibiting promoter clearance. An example of the former would include MerR, which locks RNAP in the closed complex at an operon that codes for proteins responsible for $\mathrm{Hg}^{2+}$ metabolism until a signal, $\mathrm{Hg}^{2+}$, is present (Summers 1992).

Alternatively, activation typically occurs either by stabilizing the RNAP-promoter complex or by accelerating the switch to the open complex (Roy et al. 1998). There are multiple ways that an additional factor could influence these parameters. An activator may alter the conformation of the DNA to improve the quality of the promoter, or interact with RNAP to compensate for the poor quality of the promoter, or do both. Examples of activators that improve the interaction between DNA and RNAP include MerR and BmrR, which twist the DNA in order to reposition the -10 site and the TSS in the proper orientation with RNAP to allow transcription to proceed (Heldwein et al. 2001; Brown et al. 2003). Examples of activators that accelerate the switch to the open complex include CRP and FNR, which interact with the UP element and subsequently with $\alpha \mathrm{CTD}$ to facilitate increased transcription (Wing et al. 1995; Busby et al. 1999). In this case, these activators act as "molecular velcro" by recruiting RNAP to the specific site within the DNA. 


\section{Section 1. 7. Desulfovibrio vulgaris Hildenborough (DvH)}

To study gene regulation, and particularly those genes that code for proteins responsible for sulfate reduction, $\mathrm{DvH}$ (a model SRM) was used (Figure 1-4). DvH is a model organism for studying sulfate reduction as it is genetically accessible (Keller et al. 2009; Keller et al. 2011; Sim et al. 2013) with a genome that has been sequenced (Heidelberg et al. 2004) and more recently reannotated (Price et al. 2011). DvH has a chromosome consisting of 3,570,858 bp with 3,243 protein-coding genes; it also contains a plasmid with 202,301 bp with 158 protein-coding genes. Both the chromosome and the plasmid are GC-rich (>63\%).

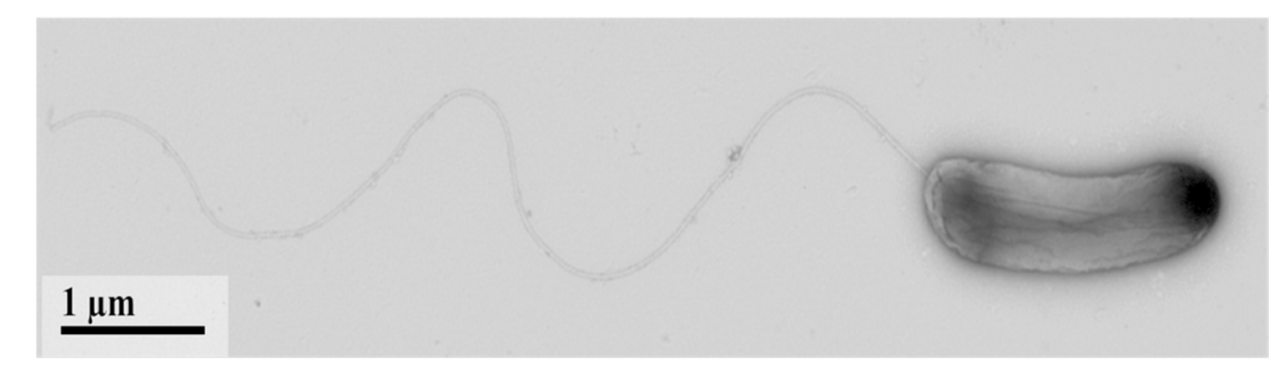

\section{Figure 1-4: Scanning Electron Microscopy (SEM) of a single DvH cell. Image provided by SR Fels with permission.}

From the genome sequence of $\mathrm{DvH}$, over 150 transcriptional regulators have been predicted (http://networks.systemsbiology.net/dvh/search/advanced) (Turkarslan et al. 2013). Interestingly, this anaerobe lacks most of the aerobic global regulators mentioned previously, although DvH does have FNR and Rex (Rex is confirmed by the work presented in this thesis). In DvH, four CRP/FNR-type global transcription regulators were annotated (Heidelberg et al. 2004) and recently characterized (Zhou et al. 2012). Each of the four homologs was determined to have distinct roles for $\mathrm{DvH}$, including responding to stress caused by $\mathrm{NaCl}$, chromate, nitrite or air. In $\mathrm{DvH}$, Rex is predicted to regulate sulfate reduction (Ravcheev et al. 2012) and is the focus of the work presented here. 
In $\mathrm{DvH}$, genes that code for proteins responsible for sulfate reduction are differentially expressed depending on the available nutrients (http://www.microbesonline.org/) (Wall et al. 2008). For instance, apsB $A$ expression was decreased in medium containing sulfite compared to expression in sulfate medium (Zane et al. 2010). Therefore, several of the predicted regulators, including Rex (Ravcheev et al. 2012), have the potential to be responsible for the observed expression changes.

$\mathrm{DvH}$ is a Gram-negative $\delta$-proteobacterium that incompletely oxidizes organic compounds including lactate, pyruvate and formate (Heidelberg et al. 2004) to acetate. Although, DvH may also oxidize $\mathrm{H}_{2}$, when an additional carbon sources is available. Additionally, DvH is capable of utilizing several electron acceptors for energy conversion, including sulfate, sulfite and thiosulfate (Barton et al. 2007). Furthermore, DvH has been associated with heavy metal reduction, including uranium reduction (Lovley 1993; Wall et al. 2006), and has been used for the purpose of bioremediation of toxic metals in the environment (Goulhen et al. 2006). Because DvH can colonize concrete and ferrous piping used in the oil industry, which leads to corrosion and "souring" of the oil, this bacterium poses a substantial problem for the industrialized world (Beech et al. 2007). Therefore, by understanding better $\mathrm{DvH}$ and its metabolism, we can begin to control some of the environmental impacts and economic costs related to sulfate reduction.

\section{Section 1. 8. The transcriptional redox regulator Rex}

Comparative genome analyses have predicted the presence of Rex in a wide range of microbes, including Gram-negative and Gram-positive, aerobes and anaerobes. To date, Rex proteins have been studied experimentally in aerobes: Thermus aquaticus (Du et al. 1999;

Sickmier et al. 2005; McLaughlin et al. 2010), Streptomyces coelicolor (Brekasis et al. 2003) Bacillus 
subtilis (Schau et al. 2004; Larsson et al. 2005; Gyan et al. 2006; Wang et al. 2008; McLaughlin et al. 2010; Wang et al. 2011), Staphylococcus aureus (Pagels et al. 2010), Streptococcus mutans (Bitoun et al. 2011; Bitoun et al. 2012) and Enterococcus faecalis (Vesic et al. 2013); and the anaerobes Thermatoga maritima (Ravcheev et al. 2012), Clostridium acetobutylicum (Wietzke et al. 2012) and Desulfovibrio alaskensis G20 (Kuehl et al. 2014). This work will add to our understanding of the role of Rex in SRM.

Functional Rex proteins contain an N-terminal DNA-binding domain (winged helix), a dimerization domain (three-dimensional domain swap), and a C-terminal Rossman fold (Sickmier et al. 2005; McLaughlin et al. 2010) (Figure 1-5). The latter apparently functions to bind pyridine nucleotides $\left(\mathrm{NADH}\right.$ and $\left.\mathrm{NAD}^{+}\right)$. The Rossman fold typically includes a GlyX-Gly-X-X-Gly sequence that is part of a "P-loop". It also contains a key acidic residue (Asp112 in T. aquaticus) that discriminates $\operatorname{NAD}(\mathrm{H})$ from their phosphorylated forms.

The consensus binding sequence for Rex, TT'TGTGAAATAT'TTCACAAA, has been compiled from comparisons of over 100 genomes (Ravcheev et al. 2012). The sequence logo of the Rex DNA-binding site, specific to Desulfovibrionales, has been determined (Figure 1-6) (Novichkov et al. 2012). The sequence contains an inverted repeat ("GTG" and "CAC", underlined), which is well-conserved as represented by bigger letters at positions 3-5 and 1416. Rex functions as a homodimer, and the inverted repeat is the minimum sequence for Rex binding, as observed in S. coelicolor (Brekasis et al. 2003). 


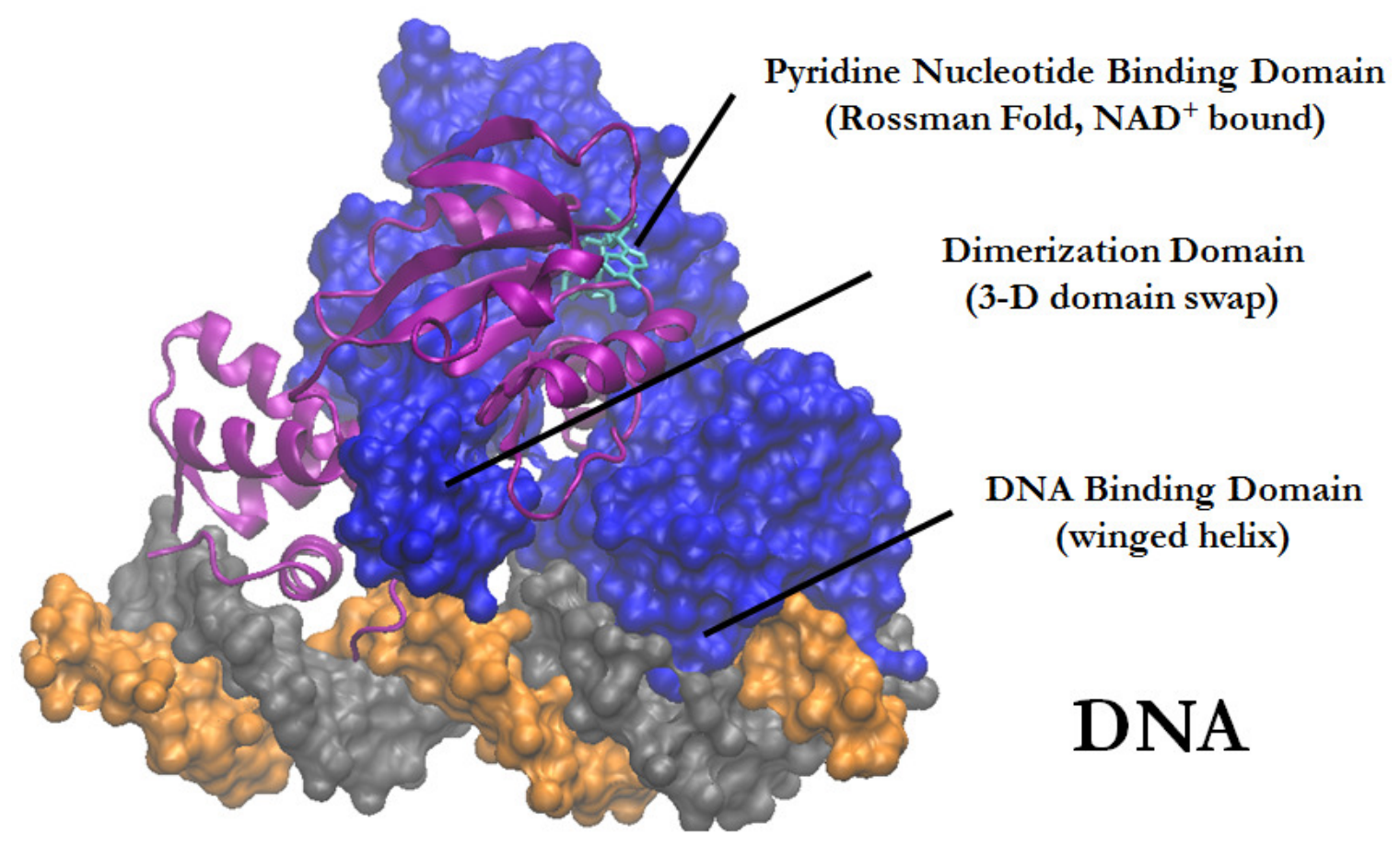

Figure 1-5: Crystal structure of Rex bound to DNA from T. thermophiles HB27. Adapted from McLaughlin et al. (2010) (pdb:3IKT). Rex monomers shown in blue (surface representation) and purple (new-cartoon representation); DNA helix in grey and orange (surface representation); and $\mathrm{NAD}^{+}$in cyan (stick representation). Modelling done by Christensen GA with Visual Molecular Dynamics (VMD) software.

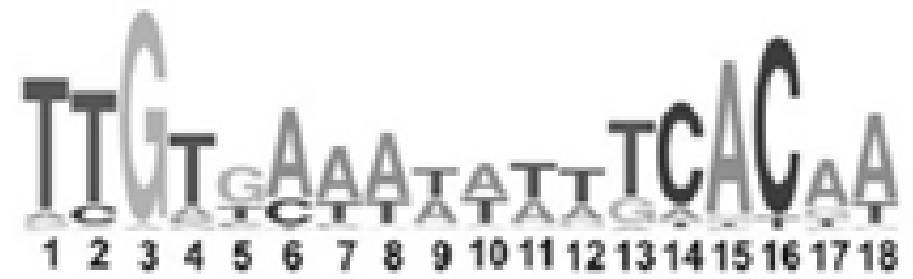

Figure 1-6: Sequence logo of the Rex DNA-binding site in Desulfovibrionales. Nine Rex regulators were analyzed from proteobacteria lineage. Figure adapted from Ravcheev et al. (2012). 
Targets for Rex (i.e. the Rex regulon) typically include genes that code for proteins involved in NADH oxidation, and Rex acts as a transcriptional repressor when the $\mathrm{NADH} / \mathrm{NAD}^{+}$ratio is low (Gyan et al. 2006; Ravcheev et al. 2012). Rex from T. aquaticus, expressed in E. coli, was crystallized in the presence of NADH and $\mathrm{NAD}^{+}$, respectively, and the structures were solved (Sickmier et al. 2005; McLaughlin et al. 2010). From these in vitro assays it was clear that Rex underwent structural changes that modulated the binding activity between Rex and a consensus DNA sequence. Specifically, when Rex bound NADH, the resulting conformation of Rex was no longer able to interact within the major groove of DNA, and therefore, would no longer repress (McLaughlin et al. 2010) (Figure 1-7). Specific amino acids (R16, Y98 and D188 in T. aquaticus) have been shown to be responsible for this switch (McLaughlin et al. 2010) (Figure 1-8). By exchanging interactions between these select amino acids, which is modulated by the bound pyridine nucleotide (NADH or $\mathrm{NAD}^{+}$), a global reorientation occurs. These results were consistent with in vitro protein-DNA interaction assays performed on an upstream DNA sequence of adhE2 in Clostridium acetobutylicum. In these assays, increased expression of adbE2 was observed in a strain deleted for rex (Wietzke et al. 2012). 

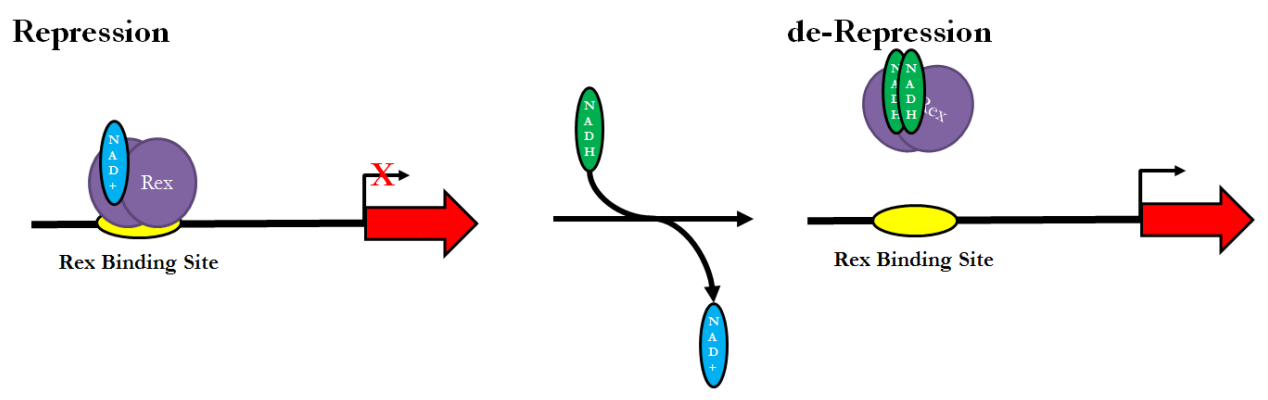

Figure 1-7: Binding activity of $\operatorname{Rex}$ is regulated by $\operatorname{NAD}(\mathbf{H})$. Rex homodimer binds to promoter sequence and represses downstream gene. Upon binding by NADH, which displaces $\mathrm{NAD}^{+}$, a conformational change occurs that prevents interaction between Rex and the DNA, allowing expression of the downstream gene.
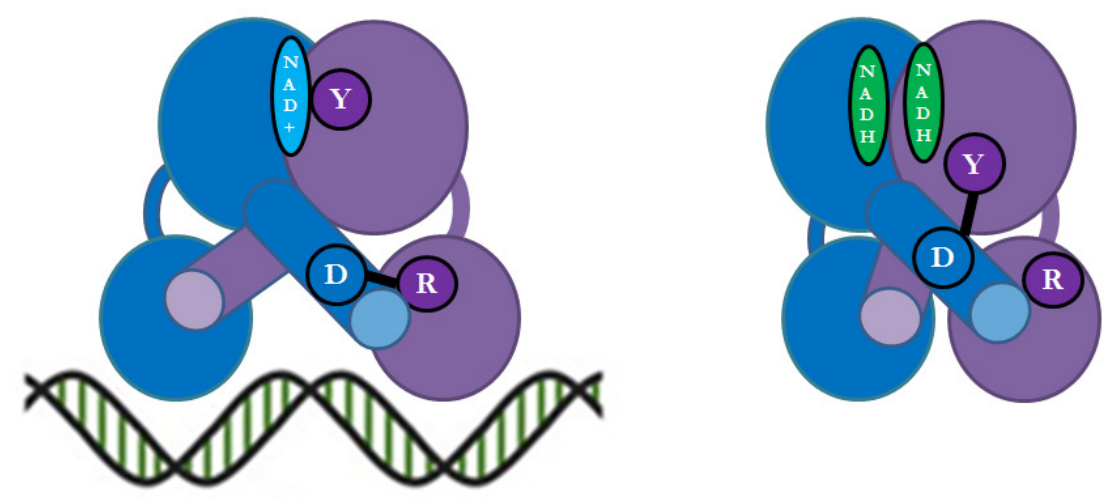

Figure 1-8: Simplified schematic of Rex conformational switch. Adapted from McLaughlin et al. (2010). Monomers of the Rex homodimer are shown in purple and blue. Amino acids (1-letter designation) thought to be responsible for the shift in conformation are shown, with the bond linking the amino acids represented by a black line. (LEFT) Rex bound in the presence of $\mathrm{NAD}^{+}$or (RIGHT) Rex unbound in the presence of NADH to DNA. 
Examination of expression data in a SRM closely related to DvH, D. alaskensis G20, revealed increased transcript expression for sat in a strain deleted for rex; however, minimal differences were observed for $a p s B A$ and $d s r A B D$ (Kuehl et al. 2014). The increase in expression for sat, which codes for the first enzyme in the process of sulfate reduction, but not aps $B A$ and $d s r A B D$, provides support for the role of Rex as a transcriptional repressor for early steps in sulfate reduction. Interestingly, $d h c A-r n f C D G E A B F$, which is predicted to be within the Rex regulon (Ravcheev et al. 2012), was reported to be decreased in the Rex mutant (Kuehl et al. 2014), although less than twofold. This was interpreted by the authors to mean that Rex may act not only as a repressor, but as an activator as well, although the authors also point out that this operon is predicted to be under complex regulation by other regulators as well. Therefore, these characteristics for Rex were considered in the examination of Rex in DvH.

\section{Section 1.9. Regulation of sulfate reduction genes by $\operatorname{Rex}_{\mathrm{DvH}}$}

In DvH, DVU0916 ( $\left.\operatorname{Rex}_{\mathrm{DvH}}\right)$ was predicted to code for a Rex protein and was hypothesized to have a role in the reduction of sulfate (Ravcheev et al. 2012). It was proposed that $\operatorname{Rex}_{\mathrm{DvH}}$ regulated over 50 genes (from 14 separate operons) that code for proteins within the energy conversion pathway (Table 1-1), and the following in particular: sat, apsBA,dsr $A B D, p p a C$ and $a d k$, all of which code for proteins that function within the cytoplasm to reduce sulfate to sulfide. Additionally, Rex $x_{\text {DvH }}$ was also predicted to regulate genes that code for many proteins involved in electron transfer within the periplasmic membrane, including the quinone reductase $(q r A B C D)$ and dissimilatory sulfite reductase (dsrMKJOP). 


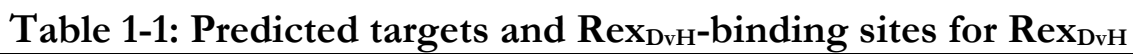

\begin{tabular}{|c|c|c|}
\hline Gene* & ID & Predicted Rex ${ }_{\mathrm{DvH}-\text { Binding Sites }}$ \\
\hline atpI & DVU0920 & CTTGTGAACGATTGCACGAA \\
\hline $\operatorname{qrc} A$ & DORF6830 & TTC $\overline{\text { TTGAAATATTTCACCTT }}$ \\
\hline $\operatorname{coo} M$ & DVU2286 & ATTEGGAATCGA $\overline{\text { TTCACAAA }}$ \\
\hline$d s r M$ & DVU1292 & 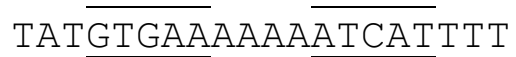 \\
\hline$d h c A$ & DVU2791 & CTTGTGAAATAATGTTCTTT \\
\hline adk & DVU1932 & 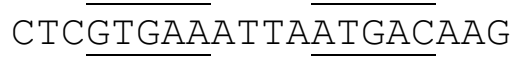 \\
\hline sat & DVU1295 & TтTGTAAATтTтTTCACAAG \\
\hline$a p s B$ & DVU0846 & АTT $\overline{\text { TTTAATTCCATCACAAG }}$ \\
\hline$p p a C$ & DVU1636 & ATTGTGCTATTTGGCACAAA \\
\hline bys $B$ & DVU1917 & CGA $\overline{\text { GCTATATATTTCACAAA }}$ \\
\hline atpF1 & DVU0780 & TTTGAGCTTTAATTCACAAC \\
\hline$d s r A$ & DVU0402 & TTTGTCCAAAAA $\overline{A T C A C G A G ~}$ \\
\hline bupB & DVU1795 & TTTGGGAAAAAA $\overline{\text { AGCGCAAG }}$ \\
\hline Null & DVU2058 & стTATGGTGTTTTACACAAA \\
\hline
\end{tabular}

* Only the first gene in the operon is displayed. Target list predicted from Ravcheev et al. (2012).

The properties described for Rex (i.e. a regulon containing genes that code for proteins responsible for energy conversion and a regulator that responds to the $\mathrm{NADH} / \mathrm{NAD}^{+}$ratio) would be those expected for a regulator of genes that code for proteins that function in sulfate reduction for SRM, as dissimilatory sulfate reduction is the major pathway for energy conversion. Therefore, a Rex homolog in $\mathrm{DvH}$ is a reasonable candidate regulator. By differentially binding $\mathrm{NAD}^{+}$and $\mathrm{NADH}$ and de-repressing genes that code for proteins that oxidize NADH when sulfate reduction is slowed (i.e. NADH levels accumulate), Rex may maintain $\mathrm{NADH} / \mathrm{NAD}^{+}$levels by altering gene expression for those proteins that can restore steady-state respiration rates (Gyan et al. 2006). Using this paradigm, we proposed that Rex might be involved in the switch between respiration and fermentation in SRM and would be necessary for adaptation to fluctuating electron acceptor concentrations within the environment. Additionally, Rex may be responsible for directing electron flow between the cytoplasm and periplasm. 


\section{Section 1. 10. Project Aims}

\section{Bioinformatically confirm Rex in DvH:}

DVU0916 was recently annotated as the redox sensor Rex based on amino acid sequence identity to known Rex proteins and consensus Rex-binding sites identified upstream of many genes that code for proteins responsible for sulfate reduction processes in $\mathrm{DvH}$ (Ravcheev et al. 2012). The amino acid sequence was therefore further analyzed through modelling techniques to justify pursuing the role of this regulator in sulfate reduction. This work is presented in Chapter 2, and the aims for this section are as follows:

- Compare protein sequence of putative $\operatorname{Rex}_{\mathrm{DvH}}$ to characterized Rex proteins.

- Model (“thread”) Rex $x_{\text {DvH }}$ to characterized Rex proteins in multiple conformations.

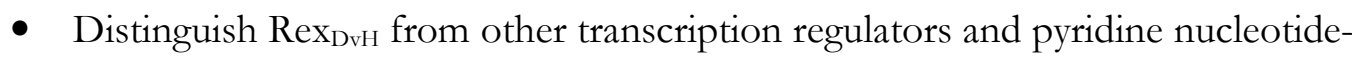
dependent enzymes.

- Assess model quality (QMEAN4 and Global Model Quality Estimation [GMQE]) based on structural descriptors.

\section{Determine the role of $\operatorname{Rex}_{\mathrm{DvH}}$ on sat expression:}

Rex is hypothesized to control, in part, the process of sulfate reduction in DvH. Sulfate adenylyl transferase (encoded by sat), which codes for the first enzyme in the sulfate reduction pathway, was analyzed for its regulation by $\operatorname{Rex}_{\mathrm{DvH}}$. This work is presented in Chapter 3, and the aims for this section are as follows:

- Construct a strain deleted for rex as well as several sat promoter mutation strains.

- Compare growth kinetics of constructed mutants to a parental strain.

- Restore phenotype of rex deletion strain by complementation. 
- Construct and purify protein from an inducible overexpression strain for Rex $\operatorname{DvvH}_{\mathrm{Dv}}$.

- Confirm interaction between Rex $x_{\mathrm{DvH}}$ and sat promoter with in vitro assays.

- Prove NADH, and no other pyridine nucleotide, modulates Rex $\mathrm{D}_{\mathrm{D} v \mathrm{H}}$ activity.

- Confirm interaction between $\operatorname{Re}_{\mathrm{DvH}}$ and its predicted targets with a more highthroughput in vitro assay.

- Determine the transcription start site(s) for sat with in vitro assays.

- Elucidate sat expression among strains and growth conditions.

- Prove Rex $x_{\text {DvH }}$ represses sat.

\section{Further characterize the function of Rex during growth:}

The role of Rex has been shown to be important for sensing changes in the rate of respiration through the ratio $\mathrm{NADH} / \mathrm{NAD}^{+}$, and controlling for gene expression to recycle the excess NADH that builds up during decreased rates of respiration (Sickmier et al. 2005). The Rex mutant was therefore examined for phenotypic differences in growth compared to a parental strain. In addition, expression differences for several genes (and proteins) responsible for sulfate reduction processes were examined. This work is presented in Chapter 4, and the aims for this section are as follows:

- Determine whole-genome gene fitness differences between the parental and Rex mutant strains for cultures respiring sulfate with lactate.

- Assess growth kinetics for the Rex mutant by pairing different electron donors/acceptors.

- Measure transcript (and protein) levels for select sulfate reduction genes (enzymes).

- Propose a model for the role of Rex in sulfate reduction, with consideration to the Rex mutant being impaired for growth when thiosulfate is the sole electron acceptor. 
Chapter 2: $\quad$ Bioinformatic determination of $\operatorname{Rex}_{\mathrm{DvH}}$ 


\section{Section 2.1. Overview}

The process of sulfate reduction is well documented, but the regulation of the genes that code for the proteins responsible for these functions is not well known. The protein encoded by DVU0916 is predicted, based on comparative genomic analyses, to be a transcriptional repressor, Rex (Ravcheev et al. 2012), which may control part of the process of sulfate reduction. Therefore, additional computational techniques were applied to the protein sequence of DVU0916 to determine if experimental characterization of this protein would be justifiable.

\section{Section 2.2. Sequence comparison}

To examine the possible role of the protein encoded by DVU0916, the protein sequence was first compared to other known Rex proteins experimentally studied (Brekasis et al. 2003; Sickmier et al. 2005; Gyan et al. 2006; Ravcheev et al. 2012). DVU0916 shares greater than $30 \%$ protein identity with the most well-studied Rex homologs to date, including T. aquaticus (TT_C1293, 42\% identity), S. coelicolor (SCO3320, 33\% identity) and B. subtillus (BSU05970, $33 \%$ identity) (Table 2-1).

Table 2-1: Protein sequence identity among Rex homologs

\begin{tabular}{|r|c|c|c|c|}
\hline & T. aquaticus & S. coelicolor & B. subtilis & DvH \\
\hline T. aquaticus & ID & $34.8 \%$ & $33.7 \%$ & $46.0 \%$ \\
\hline S. coelicolor & & ID & $30.5 \%$ & $33.3 \%$ \\
\hline B. subtilis & & & ID & $32.5 \%$ \\
\hline DvH & & & ID \\
\hline
\end{tabular}

DVU0916 protein sequence was aligned to the Rex proteins discussed above (Figure 2-1). Examination of the aligned sequence revealed that the most important features, including the DNA recognition helix ( $\alpha 3)$, the conserved aspartate that is responsible for distinguishing NADH from NADPH, and the Gly-X-Gly-X-X-Gly sequence of the P-loop 
that is common for pyridine nucleotide binding, are well conserved among the sequences, including DVU0916. Interestingly, two of three highly conserved sites within the protein thought to be responsible for the observed transition between a DNA-bound and unbound form (McLaughlin et al. 2010) were different for DvH. In the strains observed by McLaughlin et al. (2010), the amino acid (Aa) sequence is "RYD” (R16, Y98, D188, numbered for T. aquaticus); however, in DvH it is "QHD” (Q22, H104, D196, numbered for DvH). In fact, most sequenced strains of Desulfovibrio have the "QHD” triad (Figure 2-2). Therefore, DVU0916 would appear to be a Rex homolog, but the mechanism for its activity may be slightly different.

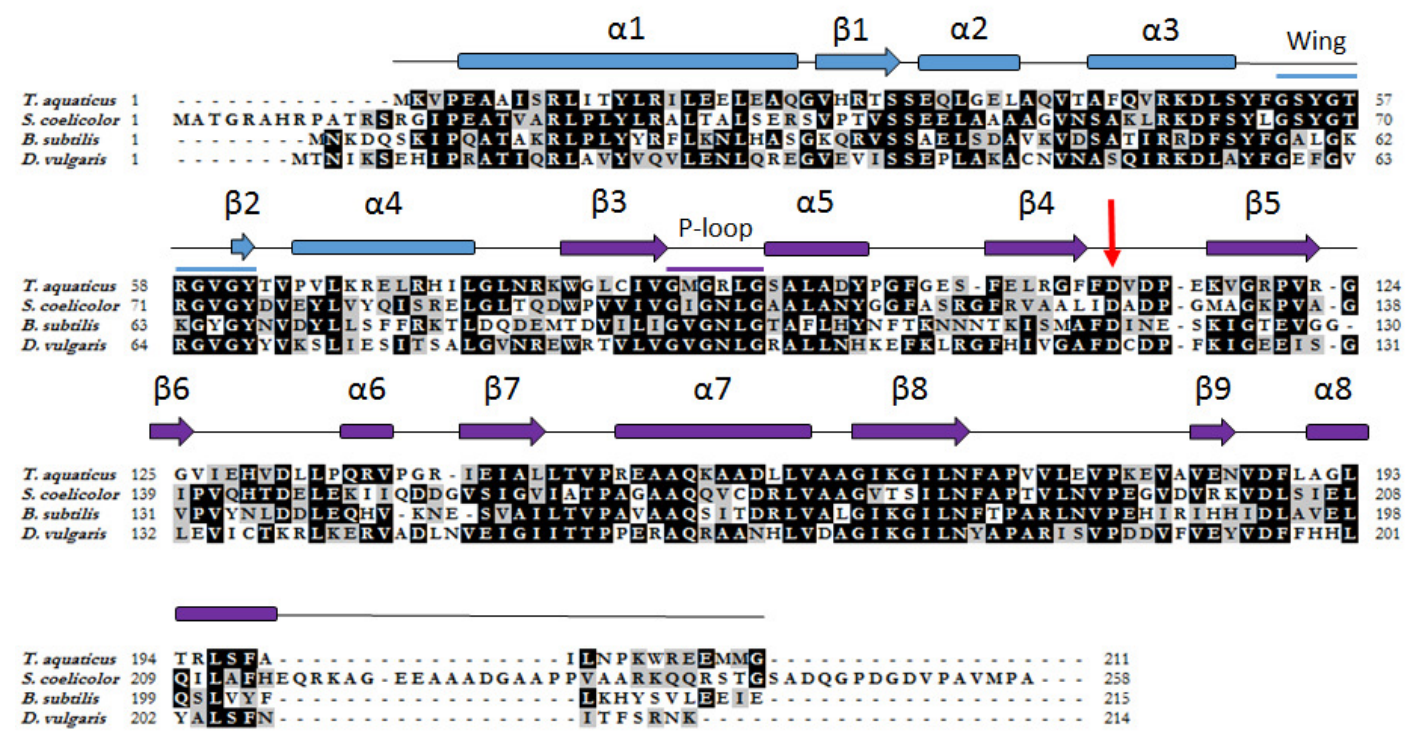

Figure 2-1: Protein sequence alignment of Rex proteins. Sequence identity (black) or similarity (grey) highlighted. Secondary structures are marked with rectangle (alpha helix) or arrow (beta sheet). The DNA-binding domain is in blue, with the ligand-binding domain (Rossmann fold) in purple. The conserved aspartate residue (D), which distinguishes $\mathrm{NAD}(\mathrm{H})$ from $\mathrm{NADP}(\mathrm{H})$, is shown with a red down-facing arrow. Figure adapted from Sickmier et al. (2005). 


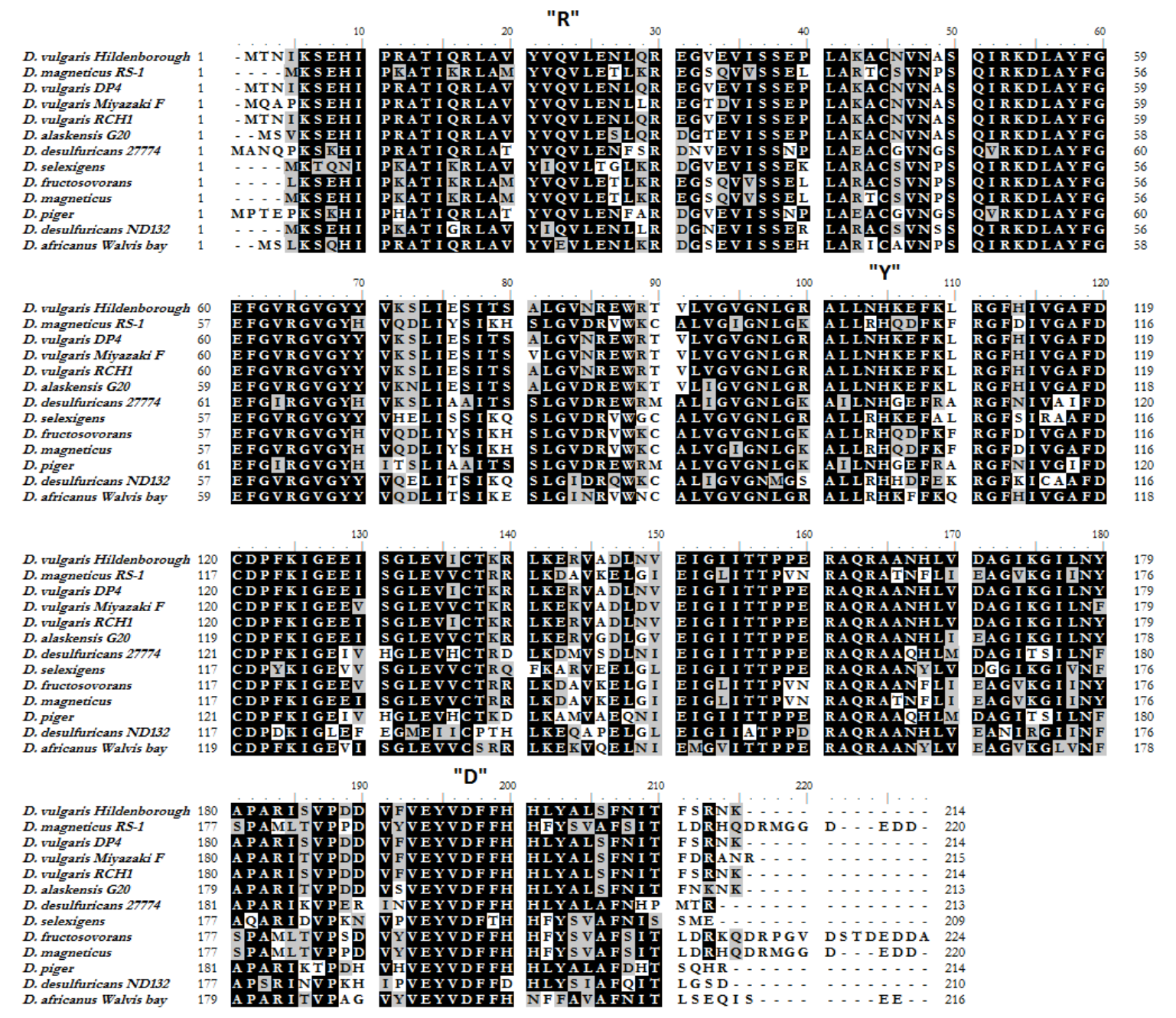

Figure 2-2: Sequence alignment of Rex homologs throughout

Desulfovibrionales. Amino acid sequence alignment of the thirteen strains,

with identity (black) or similarity (grey) highlighted. The position of the Aa's

(1-letter designation, "RYD”) that are significant for the conformational shift

is marked. 


\section{Section 2. 3. Modelling}

To further assess the likelihood of DVU0916 being a Rex protein, additional computational analyses, including protein structure homology-modelling, were performed. To that end, DVU0916 protein sequence was analyzed for its ability to fold (i.e. "thread") to previously solved structures by SWISS-MODEL (Arnold et al. 2006; Guex et al. 2009; Kiefer et al. 2009; Biasini et al. 2014) (Table 2-2). The quality of the model was assessed by two scores: QMEAN4 and the Global Model Quality Estimation (GMQE). In brief, QMEAN4 is a composite score of four structural descriptors (C $\beta$ interaction, all-atom pair-wise, solvation, and torsion angle energies) that are used to compare to scores calculated from a series of Xray structures within the database, with acceptable values greater than -3.0. The GMQE is a quality estimation that includes the target-template alignment, and is expressed as a value ranging from 0-1, with higher numbers reflecting greater reliability and acceptable values greater than 0.75 . Pairwise protein sequence alignments are performed by LALIGN server with BLOSUM50 algorithm (Goujon et al. 2010; McWilliam et al. 2013).

\section{Section 2. 3. 1. Automated modelling}

The protein sequence of DVU0916 was first automated to the SWISS-MODEL database to identify the "best-fit" template (Table 2-2). The resulting template was TTHA1657, an ATrich DNA binding protein from T. thermophilus HB8 (pdb 2DT5, (Nakamura et al. 2007)) that shared 46\% sequence identity across 201 Aa (QMEAN4=-1.84; GMQE=0.78). This protein, since its initial crystallization, has also been determined to be a Rex protein as part of a separate study (Ravcheev et al. 2012). Automated alignments are considered sufficiently reliable when the protein sequence identity between the template and target share $>50 \%$ sequence identity, and as the two being examined share $\sim 45 \%$ they should be scrutinized 
more carefully. However, the QMEAN4 and GMQE scores are within the acceptable range. The fact that the best-fit model determined via automated modelling was a DNA-binding protein is strong evidence for the role of DVU0916 as a transcription factor. Interestingly, TTHA1657 crystallized with the ligand $\mathrm{NAD}^{+}$, and the molecules $\mathrm{NAD}^{+}$and $\mathrm{NADH}$ are known to modulate the activity of Rex proteins. However, based on the model, part of the pyridine nucleotide binding-domain in DVU0916 is not conserved between the two sequences, specifically, $\beta 5$ (Figure 2-3). Therefore, DVU0916 was considered a potential transcription regulator, but may not sense pyridine nucleotides, and would need to be analyzed further.
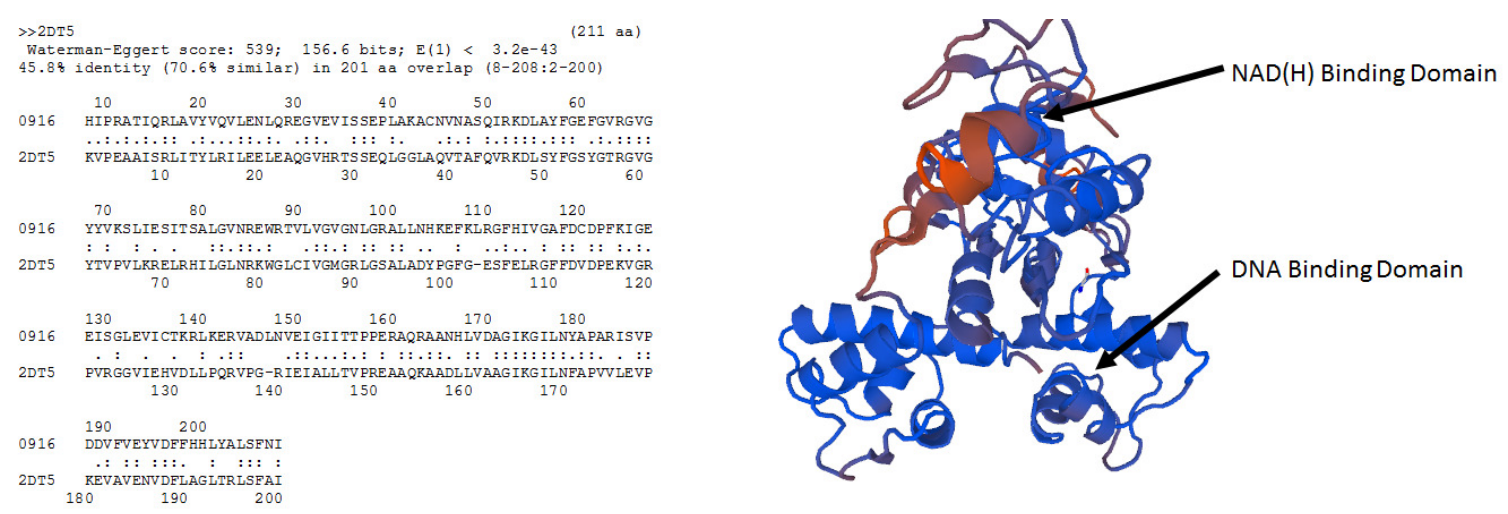

\section{Figure 2-3: DVU0916 protein sequence auto-aligned to pdb:2DT5.}

Protein sequence alignment shown on left, with the modelled structure on the right. DVU0916 is threaded to each monomer, chains A and B of pdb:2DT5. Estimated per-residue inaccuracies were visualized with a color gradient, with blue being more reliable and red being less reliable. 
Table 2-2: Model quality assessment for DVU0916

\begin{tabular}{|c|c|c|c|c|c|c|c|c|}
\hline Alignment & $\begin{array}{c}\mathrm{C} \beta \\
\text { interaction } \\
\text { energy }\end{array}$ & $\begin{array}{l}\text { All-atom } \\
\text { pairwise } \\
\text { energy }\end{array}$ & $\begin{array}{c}\text { Solvation } \\
\text { energy }\end{array}$ & $\begin{array}{l}\text { Torsion } \\
\text { angle } \\
\text { energy }\end{array}$ & QMEAN4 & GMQE & & $\begin{array}{l}\text { Protein } \\
\text { Sequence } \\
\text { Identity }\end{array}$ \\
\hline \multicolumn{9}{|l|}{ Automated } \\
\hline $\begin{array}{l}\text { DVU0916 automated to pdb: 2DT5 } \\
\text { Aligned }\end{array}$ & 0.20 & -1.22 & -2.19 & -1.13 & -1.84 & 0.78 & $\mathrm{P}$ & $46 \%$ across $201 \mathrm{Aa}$ \\
\hline DVU0916 to pdb:3IKT & 0.30 & -0.70 & -2.52 & -0.83 & -1.72 & 0.77 & $\mathrm{P}$ & $46 \%$ across $201 \mathrm{Aa}$ \\
\hline DVU0916 to pdb:3IKT, TF domain & 0.39 & -0.20 & -1.00 & 0.37 & -0.03 & 0.78 & $\mathrm{P}$ & $50 \%$ across $66 \mathrm{Aa}$ \\
\hline DVU0916 to pdb:3IKT, ligand domain & -1.41 & -2.17 & -2.14 & -1.98 & -2.87 & 0.77 & $\mathrm{P}$ & $46 \%$ across $127 \mathrm{Aa}$ \\
\hline DVU0916 to pdb:1XCB & -0.83 & -2.18 & -4.02 & -3.24 & -4.68 & 0.78 & $\mathrm{~F}$ & $46 \%$ across $201 \mathrm{Aa}$ \\
\hline DVU0916 to pdb:1XCB, TF domain & 0.14 & -0.48 & -1.43 & -1.58 & -1.79 & 0.79 & $\mathrm{P}$ & $50 \%$ across $66 \mathrm{Aa}$ \\
\hline DVU0916 to pdb:1XCB, ligand domain & -2.48 & -2.97 & -3.57 & -3.40 & -4.92 & 0.77 & $\mathrm{~F}$ & $46 \%$ across $127 \mathrm{Aa}$ \\
\hline DVU0916 to pdb:2ZCW, TF domain & -1.47 & -0.98 & -1.16 & -3.72 & -4.00 & 0.72 & $\mathrm{~F}$ & $37 \%$ across $70 \mathrm{Aa}$ \\
\hline DVU0916 to pdb:3OIO, TF domain & -1.26 & -1.62 & -3.16 & -3.94 & -4.99 & 0.60 & $\mathrm{~F}$ & $25 \%$ across $76 \mathrm{Aa}$ \\
\hline DVU0916 to pdb:WVO, TF domain & -1.69 & -1.06 & -2.42 & -1.69 & -3.10 & 0.44 & $\mathrm{~F}$ & $23 \%$ across $56 \mathrm{Aa}$ \\
\hline DVU0916 to pdb:1BIA, TF domain & -1.08 & -1.29 & -1.40 & -0.42 & -1.30 & 0.40 & $\mathrm{~F}$ & $28 \%$ across $48 \mathrm{Aa}$ \\
\hline DVU0916 to pdb:1GCU, ligand domain & -1.89 & -2.45 & -5.23 & -2.25 & -5.01 & 0.62 & $\mathrm{~F}$ & $23 \%$ across $102 \mathrm{Aa}$ \\
\hline DVU0916 to pdb:1DRW, ligand domain & -4.62 & -4.29 & -4.51 & -6.80 & -9.01 & 0.64 & $\mathrm{~F}$ & $31 \%$ across $121 \mathrm{Aa}$ \\
\hline DVU0916 to pdb:2OHX, ligand domain & -5.52 & -4.36 & -3.79 & -3.51 & -6.26 & 0.71 & $\mathrm{~F}$ & $41 \%$ across $128 \mathrm{Aa}$ \\
\hline DVU0916 to pdb:1J8F, ligand domain & -4.18 & -3.23 & -4.37 & -6.62 & -8.96 & 0.71 & $\mathrm{~F}$ & $39 \%$ across $87 \mathrm{Aa}$ \\
\hline
\end{tabular}

$\mathrm{P}$ and $\mathrm{F}$ denote Pass or Fail, respectively, for the model generated. 


\section{Section 2. 3. 2. Aligned modelling}

In order to compare how well DVU0916 protein resembled a characterized Rex protein, DVU0916 protein sequence was modelled to two unique conformations of the Rex protein from T. aquaticus. First, DVU0916 was compared to Rex co-crystallized with $\mathrm{NAD}^{+}$in a conformation that allowed for interaction with DNA (pdb:3IKT) (McLaughlin et al. 2010)), and second, to Rex co-crystallized with NADH in a form that was unable to interact with DNA (pdb:1XCB) (Sickmier et al. 2005)) (Table 2-2). DVU0916 shared 46\% protein identity across $201 \mathrm{Aa}$ (6e-52) with Rex from T. aquaticus. Additionally, the amino terminal (N-term) DNA-binding (residues 1-81) and carboxy terminal (C-term) dimerization and pyridine dinucleotide (ligand)-binding domains (residues 82-214) of DVU0916 were aligned separately, in order to better refine the modelling for each domain (Table 2-2). Interestingly, DVU0916 modelled best to the DNA-bound form (pdb:3IKT), and less well to the unbound form (pdb:1XCB) (Figure 2-4).

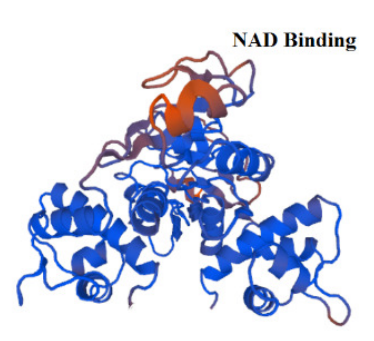

DNA Binding

pdb: 3IKT

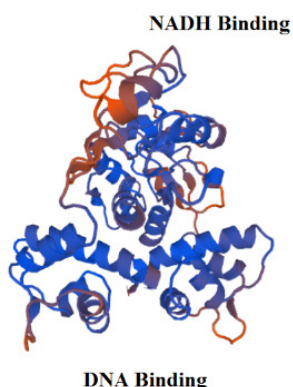

pdb: 1XCB

Figure 2-4: DVU0916 protein sequence aligned to $\operatorname{Rex}$ from $T$. aquaticus. Protein sequence of DVU0916 threaded to each monomer of (left) DNA-bound (pdb: 3IKT) (McLaughlin et al. 2010) or (right) DNA unbound (pdb: 1XCB) (Sickmier et al. 2005) conformation of Rex. Estimated perresidue inaccuracies were visualized with a color gradient, with blue being more reliable and red being less reliable. 
Examination of the quality scores for the individual domains revealed that the DNAbinding domain for each of the forms was satisfactory, but the C-terminal NAD $(H)$-binding domain for DVU0916 modelled to pdb:1XCB was the region that led to an overall poor quality score (Table 2-2). This was interpreted to mean that DVU0916 may interact with a ligand other than $\mathrm{NADH}$, or that the conformational change mechanism may be slightly different for DVU0916 than for previously characterized Rex proteins. Further examination of the individual descriptors (i.e. C $\beta$ interaction, all-atom pairwise, solvation, and torsion angle energies) used to assess quality revealed that the solvation and torsion angle energies caused the poor quality score for DVU0916 modelled to the DNA-unbound form in the presence of NADH. These results were interpreted to mean that DVU0916 may not fold in in a similar manner as known Rex proteins, but that it is likely a transcription factor nonetheless.

In order to confirm DVU0916 as a Rex protein and not another regulator, the DVU0916 protein sequence was also modelled to other known transcription factors and $\mathrm{NAD}(\mathrm{H})$-dependent enzymes. The list of proteins to examine was based on protein similarity to DVU0916, with a cut-off of $20 \%$ protein identity across 50 amino acids (Table 2-2). The shortened list included the transcription regulators: SdrP, a CRP/FNR-family regulator from T. thermophilus (pdb:2ZCW) (Agari et al. 2008); an AraC-type DNA-binding domain from Chromobacterium violaceum (pdb:3OIO) (in press), HET-S, a prion inhibitor from Podospora anserina (pdb:2WVO) (Greenwald et al. 2010), and a repressor of the biotin biosynthesis operon, BirA from E. coli (pdb:1BIA) (Wilson et al. 1992). Additionally, NAD $(\mathrm{H})$-dependent enzymes were also examined: BVR, biliverdin reductase from Rattus norvegicus (pdb:1GCU) (Kikuchi et al. 2001), dihydrodipicolinate reductase from E. coli (pdb:1DRW) (Reddy et al. 1996), LADH, liver alcohol dehydrogenase from Equus caballus 
(pdb:2OHX) (Al-Karadaghi et al. 1994), and SirT2 histone deacetylase from Homo sapiens (pdb:1J8F) (Finnin et al. 2001). Each of the additional proteins examined failed the QMEAN4 cutoff requirements. Therefore, since DVU0916 fulfilled the QMEAN4 requirements for modelling to Rex, and to no other related functional proteins, i.e. transcription regulators or $\mathrm{NAD}(\mathrm{H})$-dependent enzymes, DVU0916 was putatively considered to be Rex.

\section{Section 2.4. Discussion}

The protein sequence for DVU0916 was compared to a database of solved protein structures, including the redox sensor Rex. DVU0916 shared the greatest identity $(>30 \%)$ with previously characterized Rex proteins, and poorly $(<10 \%)$ with most others, including the transcription repressor, LacI, involved in lactose metabolism. The high sequence identity for DVU0916 compared to Rex would suggest that the two are homologous (Rost 1999). Furthermore, all domains significant for Rex functionality were observed in the DVU0916 protein sequence, including: the DNA-recognition helix ( $\alpha 3$ ), the P-loop important in the Rossman fold, and the conserved aspartate residue (Sickmier et al. 2005; Ravcheev et al. 2012; Wietzke et al. 2012) (Figure 2-1). Therefore, DVU0916 protein was predicted to interact with a similar DNA sequence as other characterized Rex proteins.

However, two differences were observed between DVU0916 protein and previously characterized Rex proteins. First, based on homology modelling, the unstructured wing domain (Gly-X-X-Gly-X-X-Gly-X-Gly) that follows the DNA-recognition helix ( $\alpha 3$ ), which is essential for the interaction between Rex and the DNA, (Sickmier et al. 2005), was flagged as being "less reliable" (Figure 2-4). Note that this is separate from the Gly-rich sequence in the Rossman fold, which is conserved. Interestingly, the winged domain in the DNAunbound form (DVU0916 modelled to pdb:1XCB) is far less reliable than in the DNA- 
bound form (DVU0916 modelled to pdb:3IKT). Further examination of DVU0916 protein sequence within this region does indicate that it is variable; however, the overall motif (i.e. glycine-rich) is maintained. Additionally, this region is an unstructured flexible region, and the variability in sequence may have no effect on the activity of the protein. Regardless, as described in the next chapter, DVU0916 protein is capable of interacting with DNA and in a similar manner as for other characterized Rex proteins. The second difference observed for DVU0916 was in the Aa sequence, within the C-terminal domain, thought to be responsible for the conformational switch that occurs (McLaughlin et al. 2010). Among the thirteen sequenced Desulfovibrio strains, none of them contained the expected "RYD" sequence, but instead the sequence was “QHD” for twelve of the thirteen examined (Figure 2-2). This series of amino acids act as a switch, that alters protein structure in response to a particular signal, NAD(H) (McLaughlin et al. 2010). Because of these differences in sequence and mediocre quality scores for DVU0916 protein modelled to the NADH domain (Figure 2-4), we initially thought that DVU0916 protein may respond to a signal different from $\mathrm{NAD}(\mathrm{H})$. However, as described in the next chapter, NADH (and no other pyridine nucleotide that was examined) prevented DVU0916 protein interaction with DNA. Therefore, it appeared that alterations in protein structure occurred in the presence of NADH in DVU0916 protein, and these differences in sequence are likely conservative substitutions that do not affect the overall function.

After examination of DVU0916 protein sequence and modelling DVU0916 to known Rex proteins, DVU0916 protein is likely to be Rex, or at least a transcription factor. Hence, this protein is worth consideration for experimental studies and will be referred to as $\operatorname{Rex}_{\mathrm{DvH}}$. In the following chapters, Rex $\mathrm{x}_{\mathrm{DvH}}$ was characterized for its role as a redox regulator of sulfate reduction in $\mathrm{DvH}$. 


\section{Chapter 3: $\quad \operatorname{Rex}_{\mathrm{DvH}}$ is a repressor of sat and its activity is modulated by NADH}

This chapter is to be published by Journal of Bacteriology, January 2015 | doi: 10.1128/JB.02083-14. It is presented here with minor revisions.

Rex (encoded by DVU0916) in Desulfovibrio vulgaris Hildenborough is a repressor of sulfate adenylyl transferase and is regulated by NADH

G.A. Christensen ${ }^{1,2}$, G.M. Zane, ${ }^{1,2}$, A.E. $\mathrm{Kazakov}^{2,3}$, X. Li ${ }^{4}$, D.A. Rodionov, ${ }^{4,5}$, P.S.

Novichkov $^{2,3}$, I. Dubchak ${ }^{2,3}$, A.P. Arkin ${ }^{2,3}$, J.D. Wall ${ }^{1,2}$

${ }^{1}$ Department of Biochemistry, University of Missouri, Columbia MO, USA

${ }^{2}$ Ecosystems and Networks Integrated with Genes and Molecular Assemblies, Berkeley CA, USA

${ }^{3}$ Physical Biosciences Division, Lawrence Berkeley National Laboratory, Berkeley, CA, USA ${ }^{4}$ Sanford-Burnham Medical Research Institute, La Jolla, CA, USA

${ }^{5}$ A.A. Kharkevich Institute for Information Transmission Problems, Russian Academy of Sciences, Moscow, 127994, RUSSIA 


\section{Section 3.1. Overview}

Rex is known to control gene expression for energy conversion processes. In DvH, DVU0916 encodes a potential Rex protein, $\operatorname{ex}_{\mathrm{DvH}}$, which is predicted to control for the process of sulfate reduction (Ravcheev et al. 2012). In particular, Rex $x_{\text {vvH }}$ may be a trigger for the switch between substrate level and oxidative phosphorylation processes. In a closely related Desulfovibrionales, D. alaskensis G20, it was observed that in a strain deleted for rex the expression of sat-encoding the enzyme that activates sulfate for the first step in its reduction - was increased (Kuehl et al. 2014). Therefore, the focus of this chapter will be on the characterization of Rex $x_{\mathrm{DvH}}$, with an emphasis on how Rex $\mathrm{x}_{\mathrm{DvH}}$ controls sat expression. To accomplish this, growth studies comparing a Rex mutant to its parental strain cultured in media that allowed for respiration or fermentation were conducted, sat expression between the strains was analyzed, the transcription start site(s) for sat were determined, and the role of $\mathrm{NAD}(\mathrm{H})$ on Rex $\mathrm{x}_{\mathrm{DvH}}$-binding to the sat promoter was examined. All results were consistent with the conclusion that $\operatorname{Rex}_{\mathrm{DvH}}$ is a redox-responsive transcriptional repressor for sat, and is regulated by NADH.

\section{Section 3. 2. rex appears to be monocistronic}

DVU0916, predicted to encode rex, is annotated to be in a six gene operon (www.microbesonline.org) and this operon is also predicted to be controlled by Rex, potentially through feedback inhibition (Figure 3-1) (Ravcheev et al. 2012). This operon is on the negative strand. A predicted Rex $\mathrm{DvH}_{\mathrm{H}}$-binding site is identified upstream of DVU0920 (Ravcheev et al. 2012). Recently, transcription start sites have been identified upstream of DVU0920, DVU0917 and rex within this operon (Price et al. 2011), which was interpreted to mean that this operon is actually three separate operons. Therefore rex is only expressed with 
the downstream gene, DVU0915, which codes for a hypothetical protein. Therefore, with rex not being co-transcribed with the upstream operon, the expression of DVU0920-18 is presumed to be controlled by Rex ${ }_{\mathrm{DvH}}$, but not DVU0917 nor rex. Interestingly, Rex from both B. subtilis and S. auereus do not appear to have a Rex-binding site upstream of rex either.

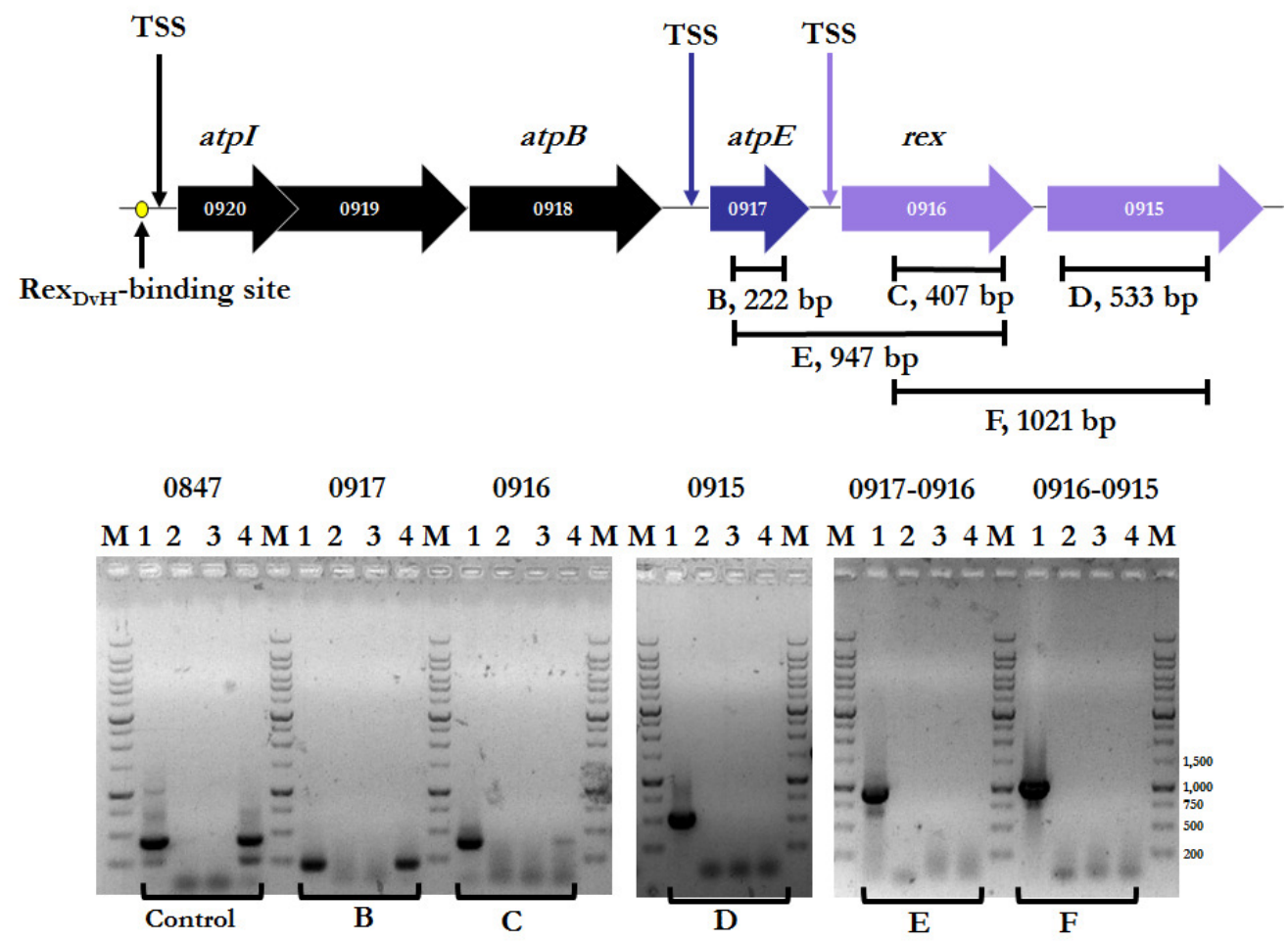

Figure 3-1: RT-PCR confirms rex to be monocistronic. TOP) operon layout for rex (DVU0916) and the surrounding genes. TSS, transcription start site. PCR fragments for RT-PCR, B-F are labelled. The expected product sizes are: DVU0917 (222 bp, upstream gene, atpE), DVU0916 (407 bp, rex), DVU0915 (533 bp, downstream gene, hypothetical), DVU0917-16 (947 bp), DVU0916-0915 (1021 bp) and DVU0917-15 (1561 bp). Color coded based on new operon layout. BOTTOM) Agarose gels of RT-PCR products. M; $1 \mathrm{~kb}$ PLUS $^{\mathrm{TM}}$ DNA ladder (Gold Biotechnology®); 1, gDNA template; 2, water; 3, no reverse transcriptase control; 4, cDNA template. 
To determine whether rex was expressed with the upstream and/or downstream genes DVU0917 and DVU0915 respectively, RT-PCR was performed on this region from a wild-type DvH culture grown by sulfate respiration with lactate (Figure 3-1). Primers were designed for each of the three genes (DVU0917, DVU0916 and DVU0915), as well as across rex and both of the two surrounding genes separately (DVU0917-DVU0916 and DVU0916DVU0915). The results of the RT-PCR (50 cycles of a $100 \mathrm{ng}$ cDNA template) revealed that rex was not transcribed with the upstream gene, as determined by bands for the single products but not the double, and no product was detected for the downstream gene DVU0915. Therefore, rex appears to be monocistronic. However, a more sensitive transcript analysis, qRT-PCR (data not shown), of DVU0915 revealed that DVU0915 is a gene, but expressed at a very low level. Additionally, DVU0915 is expressed approximately 15 -fold lower than DVU0916. This could be explained by the $33 \mathrm{bp}$ within the intergenic region between rex and DVU0915. Examination of this region did reveal potential hairpins as well as a ribosomal binding site that may influence the overall expression of DVU0915.

Therefore, additional regulatory elements, which may be influenced by growth mode (i.e. respiration versus fermentation), in addition to the previously identified transcriptional start sites determine the overall operon structure for this region.

\section{Section 3. 3. Deletion of rex increased sat expression}

To examine the role of Rex ${ }_{\mathrm{DvH}}$, a marker-exchange deletion (MED) of rex and a complemented deletion strain were constructed (Korte et al. 2014). These two strains, in addition to the parental strain, were grown by sulfate respiration in MOLS4 or pyruvate fermentation in MOYPyr. The former was expected to differ from the latter by changes in

the ratio of $\mathrm{NADH} / \mathrm{NAD}^{+}$proposed to be a signal for Rex regulation. It was assumed that lacking an inorganic terminal electron acceptor, fermenting cultures would exhibit an 
increase in NADH (Williamson et al. 1967). Examination of the growth in either medium revealed no significant differences among the three strains (Figure 3-2) (Table 3-1).
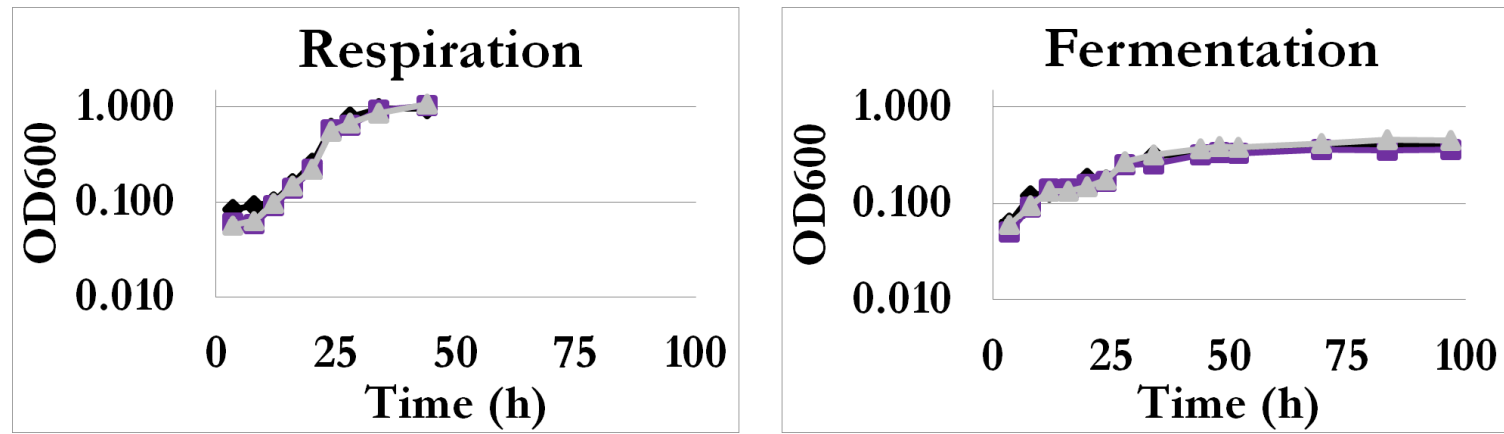

Figure 3-2: Growth studies with Rex marker-exchange deletion. Growth

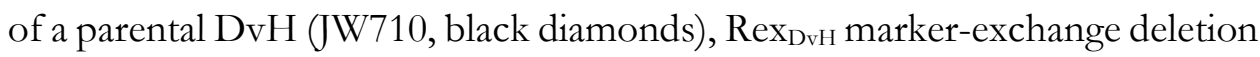
(JW3311, purple squares) and complemented Rex (JW3311(pMO3313), grey triangle) strains by sulfate respiration with $60 \mathrm{mM}$ lactate and $30 \mathrm{mM}$ sulfate or pyruvate fermentation with $60 \mathrm{mM}$ pyruvate supplemented with $0.1 \%$ (wt/vol) yeast extract and $0.5 \mathrm{mM}$ cysteine.

In parental cells, qRT-PCR of sat expression (Table 3-1) (Table A-1) revealed that sat transcription had a sevenfold increase in fermenting cultures compared with cultures respiring sulfate. In the Rex $\operatorname{Dv}_{\mathrm{Du}}$ mutant, sat expression was minimally increased when growing fermentatively but was already elevenfold increased from the parental strain while respiring sulfate, consistent with a repressor role for Rex. When this strain was complemented with a plasmid copy of rex that was transcribed from a constitutive promoter, partially restored (decreased) levels of sat transcription were observed. To eliminate the possibility that growth modes were affecting the expression of rex, transcription of that gene was examined and found to be unchanged in the parental strain and was undetected in the $\operatorname{Rex}_{\mathrm{DvH}}$ mutant as expected. Therefore, these studies provided support that $\operatorname{Rex}_{\mathrm{DvH}}$ is a transcriptional repressor for sat. 
Table 3-1: Growth and transcript analysis of Rex marker-exchange deletion

\begin{tabular}{|c|c|c|c|c|c|c|}
\hline \multirow[b]{2}{*}{ Strain } & \multirow[b]{2}{*}{ Name } & \multirow[b]{2}{*}{ Media } & \multicolumn{2}{|c|}{ Growth kinetics } & \multicolumn{2}{|c|}{ Transcript $^{a}$} \\
\hline & & & $\begin{array}{l}\text { OD600 } \\
\text { collected }\end{array}$ & $\begin{array}{l}\text { Generation } \\
\text { time }(\mathrm{h})\end{array}$ & sat & rex \\
\hline JW710 & Parental & MOLS4 & 0.25 & 6.5 & $1.0 \pm 0.2$ & $1.0 \pm 0.0$ \\
\hline JW3311 & $\Delta r e x$ & MOLS4 & 0.22 & 5.8 & $11.2 \pm 2.3$ & N.D. \\
\hline$\underset{(\mathrm{pMO} 3313)}{\text { JW3311 }}$ & $\begin{array}{c}\text { Complement } \\
\text { of rex }\end{array}$ & MOLS4 & 0.22 & 6.2 & $5.9 \pm 0.8$ & $10.9 \pm 0.0$ \\
\hline JW710 & Parental & MOYPyr & 0.18 & 52 & $7.3 \pm 1.2$ & $1.7 \pm 0.0$ \\
\hline JW3311 & $\Delta r e x$ & MOYPyr & 0.17 & 47 & $13.6 \pm 3.7$ & N.D. \\
\hline$\underset{(\mathrm{pMO} 3313)}{\mathrm{JW} 3311}$ & $\begin{array}{c}\text { Complement } \\
\text { of rex }\end{array}$ & MOYPyr & 0.18 & 38 & $6.4 \pm 0.6$ & $24.9 \pm 0.2$ \\
\hline
\end{tabular}

OD600 was monitored through growth and samples were collected for analysis by qRT-PCR at early-exponential. Approximately 100 ng of TURBO ${ }^{\mathrm{TM}}$ DNase-treated RNA was converted to cDNA and $1 \mu \mathrm{L}$ of cDNA (5 ng of RNA) was used per qRT-PCR. Each gene was assessed individually and normalized to reference genes $r p l S$ and $r p m C$. The efficiency for each gene was assessed: $r p l S=92.6 \%, r p m C=95.5 \%$, sat $=91.3 \%$ and $r e x=90.2 \%$. Error determined as standard error of the mean. ${ }^{a}$ Samples normalized to JW710 MOLS4. N.D. Not Detected.

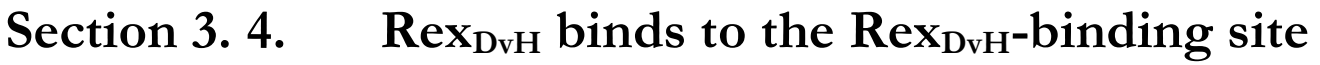

\section{upstream of sat}

As the expression levels for sat were increased by the deletion of rex, we sought to determine whether the regulation was direct or indirect. There is a putative Rex binding site located at position -150 to -131 (TTTGTAAATTT'TTTCACAAG) relative to the translational start codon for sat (Ravcheev et al. 2012). Therefore, Rex $x_{\text {DvH }}$ was purified for protein-DNA interaction studies (Figure 7-4). Four dsDNA fragments were examined for interaction with $\operatorname{Rex}_{\mathrm{DvH}}($ Figure 3-3): one upstream and one downstream of the predicted Rex binding site, and two of different sizes that contained the motif. The two fragments that contained the Rex-binding site (fragments C and D in (Figure 3-3) shifted in electrophoretic mobility when the putative Rex $x_{\text {vvH }}$ protein was present, while the other two fragments did not. These 
results confirm a direct interaction between $\operatorname{Rex}_{\mathrm{DvH}}$ protein and the putative $\operatorname{Rex}_{\mathrm{DvH}_{\mathrm{H}}-\text { binding }}$ site upstream of sat.

$\mathbf{A}$

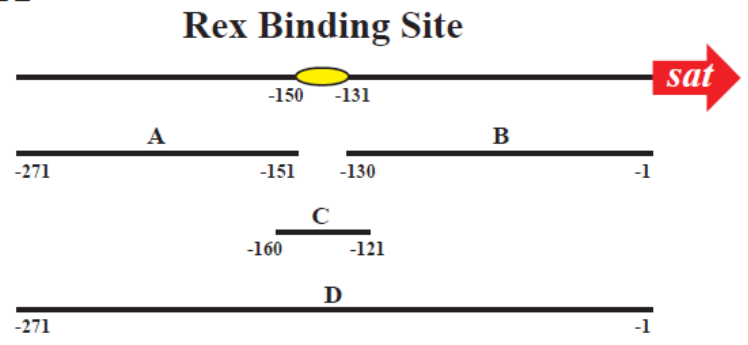

B

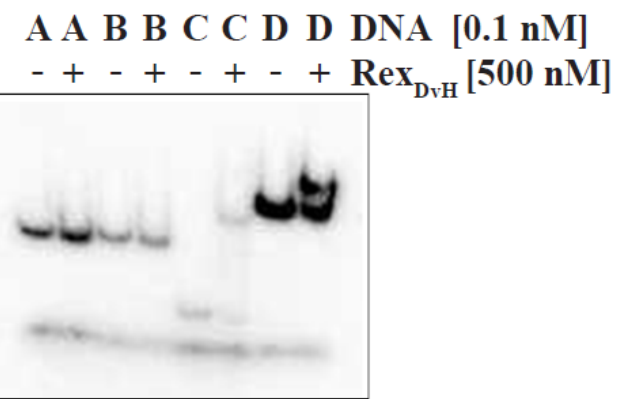

Figure 3-3: Rex $\mathrm{DvH}_{\mathrm{Dv}}$ interacts in vitro with sat promoter, shown by EMSA.

A) schematic representation of the sat promoter region approximately to scale.

The predicted Rex ${ }_{\mathrm{DvH}}-$ binding site is annotated as a yellow oval. Fragments (A, B, C, D) used in EMSA are shown with their position noted. Fragments A (121 bp), B (130 bp), and D (271 bp) were PCR amplified, while fragment C (40 bp) was generated by annealing two oligonucleotides. B) Native polyacrylamide gel of individual DNA fragments (A, B, C, D; $0.1 \mathrm{nM}, 1 \mathrm{nM}$ stock prior to column purification) without (-) or with (+) $\operatorname{Rex}_{\mathrm{DvH}}(500 \mathrm{nM})$. Equal concentration of DNA was labeled and then passed over a column to separate the fragments from the rest of the components, i.e unlabeled nucleotides. Fragment C, the smallest fragment is below the size cut-off of the column ( $\sim 100 \mathrm{bp})$ and so only a small amount of this fragment is actually recovered compared with the other three larger fragments. Each fragment was eluted in the same volume of buffer and so the concentration of this smaller fragment is considerably lower than the rest. Therefore the band intensity for fragment $\mathrm{C}$ is less than the others. The lowest band common in all lanes is the dye front. 


\section{Section 3.5. Transcript start sites for sat}

With a physical interaction observed in vitro between $\operatorname{Re}_{\mathrm{DvH}}$ and the Rex $\mathrm{x}_{\mathrm{DvH}}$-binding site upstream of sat, determination of the relative proximity of this motif to the transcript start site (TSS) for sat might provide a logical mechanism for regulation. This analysis could provide evidence that $\operatorname{Rex}_{\mathrm{DvH}}$ repressed by occluding the polymerase from interaction with the promoter region of sat. Therefore, RNA samples were isolated from parental and Rex DvH $_{\text {. }}$ strains grown by sulfate respiration or pyruvate fermentation and assessed for the TSS of sat. To determine the 5'-end of the transcript, the Rapid Amplification of cDNA Ends (RACE) technique was applied (Figure 3-4). Examination of the TSS for the parental strain revealed two unique sites, one that was identified from cells growing by sulfate respiration at $-122 \mathrm{bp}$ relative to the assumed translational start codon and one from pyruvate-fermenting cells at $64 \mathrm{bp}$. A previous study performed by 5'-RNAseq analysis also identified position -122 bp as the TSS of sat for DvH grown by sulfate respiration (Price et al. 2011). The Rex $\mathrm{Dv}_{\mathrm{Du}}$-binding site (-150 to-131 bp) is less than $10 \mathrm{bp}$ upstream of the $5^{\prime}$-end of the mRNA and therefore supports the occlusion of RNA polymerase binding for repression by $\operatorname{Re}_{\mathrm{DvH}}$ during respiration. Furthermore, a potential -35 site of a $\sigma^{70}$ promoter was identified in close proximity to this region (Figure 3-4). Interestingly, examination of the TSS for the Rex $\operatorname{DvH}_{\mathrm{D}}$ mutant growing either by respiration or by fermentation revealed the same sites as those identified for the parental strain. Because two sites were observed and deletion of rex did not reveal a change, these results suggest that factors in addition to Rex $\operatorname{DvH}_{\mathrm{v}}$ are involved in determining the TSS. 
A

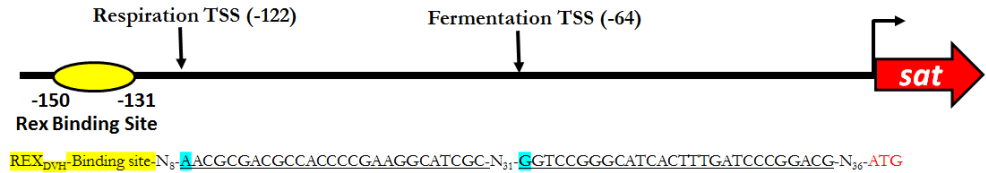

B

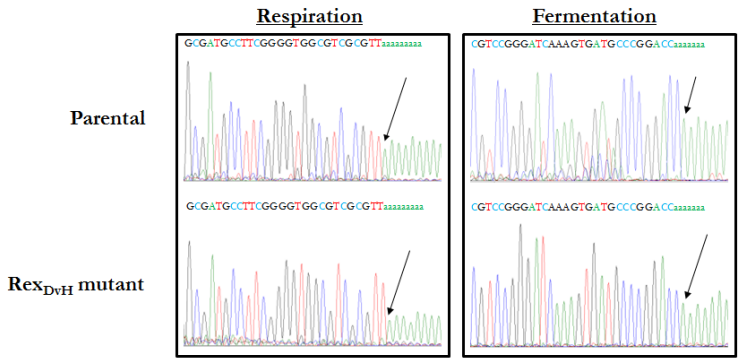

C

\begin{tabular}{|c|c|c|}
\hline & TTEACATTTTGTAAATTTTTTCACAAGACGGAATCA & \\
\hline & ATTTTGTAAATTTITTCACAAGACGGAATCA & \\
\hline & TAAAAATTTCACAA & \\
\hline iyazaki $\mathrm{F}$ & TTATGTAAAATATTTCACAAGAGGTATGCG & $G$ \\
\hline & ITC & \\
\hline & GTATTCCCT & 111 \\
\hline 38 & ITGACATITCTTTCACAAACCATTCATA & \\
\hline
\end{tabular}

Figure 3-4: Transcript start sites for sat. A) Schematic representation of the promoter region for sat with the position of the transcript start sites (TSSs) identified via 5'-RACE. Positon of the Rex $\mathrm{DvH}_{\mathrm{Du}}$-binding site is highlighted in yellow. B) Chromatogram sequence reads of PCR2 fragments of the promoter region upstream of sat from a parental and $\operatorname{Rex}_{\text {DvH }}$ mutant strain grown by respiration in MOLS4 or pyruvate fermentation in MOYPyr. Arrow denotes the position of the poly-A tail added to the end of the sequence. The 5'-end of the sequence is the first base upstream of this poly-A tail. The reads are oriented in the reverse direction based on primer design. Color code for the nucleotide bases: black, G; blue, C; red, T; green, A. C). The Rex $\operatorname{DvH}_{\text {-binding site (highlighted }}$ in yellow) and the surrounding region is shown for the promoter sequence of sat of Desulfovibrio strains, with the predicted -35 site displayed ("TTGACA", highlighted in green). TSS (respiration) for $\mathrm{DvH}$ is highlighted in blue. 


\section{Section 3. 6. Effect of NADH concentrations on $\operatorname{Rex}_{\mathrm{DvH}}$ function}

Rex proteins contain a pyridine nucleotide binding domain that has been shown in other bacteria to interact with $\mathrm{NAD}^{+}$or NADH and influence regulation (Sickmier et al. 2005;

Pagels et al. 2010). To determine the role of pyridine nucleotide interaction with Rex $\mathrm{x}_{\mathrm{DvH}}$, DNA-binding assays were performed with purified $\operatorname{Re}_{\mathrm{DvH}}$ protein in the presence of $\mathrm{NAD}^{+}$, $\mathrm{NADH}, \mathrm{NADP}^{+}$or NADPH at 0.1 or $1.0 \mathrm{mM}$ concentration (Figure 3-5) and a DNA fragment containing the Rex $\operatorname{DvH}_{-}$binding site (fragment C, Figure 3-3)). Addition of NAD ${ }^{+}$ appeared to have little effect on binding at either concentration compared with Rex $x_{D v H}$ and DNA alone; whereas, NADH disrupted the interaction at both concentrations tested. $\mathrm{NADP}^{+}$did not appear to have any effect on the binding event, and NADPH disrupted binding only at high, presumably non-physiological, concentrations. 


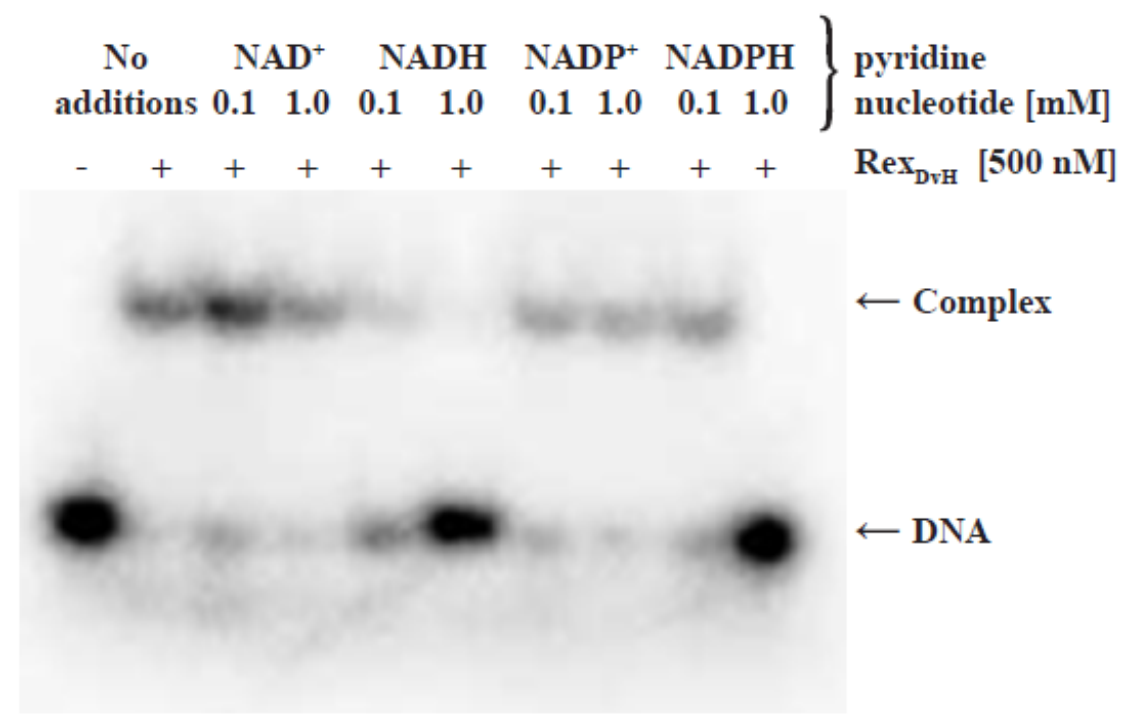

Figure 3-5: NADH disrupts interaction between $\operatorname{Rex}_{\mathrm{DvH}}$ and the $\operatorname{Rex}_{\mathrm{DvH}^{-}}$ binding site. Electrophoretic assay demonstrating the effect of pyridine nucleotide on Rex ${ }_{\mathrm{DvH}}$ binding. Fragment C (40 bp, including Rex $\operatorname{Dv}_{\mathrm{DvH}}-\mathrm{binding}$ site upstream of sat) at $0.1 \mathrm{nM}(1 \mathrm{nM}$ prior to column purification) was incubated with $\operatorname{Rex}_{\mathrm{DvH}}(500 \mathrm{nM})$ in the presence of a low or high concentration of specified pyridine nucleotide. Location of the DNA or protein-DNA complex is noted by arrows. 


\section{Section 3. 7. Essential nucleotides of the Rex-binding motif}

To determine the key bp(s) recognized by $\operatorname{Rex}_{\mathrm{DvH}}$ for binding, a number of mutations within the motif upstream of sat were altered and binding studies were performed (Table 3-2). The strategy for the base alterations was to make transitional mutations (i.e. purine to purine (A $\nleftarrow \rightarrow \mathrm{G})$ or pyrimidine to pyrimidine $(\mathrm{C} \leftarrow \rightarrow \mathrm{T})$ ) for the most conserved bases of the

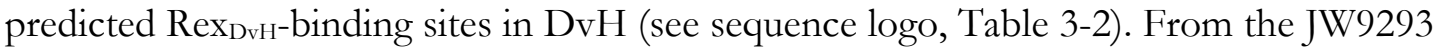
deletion strain $\left(\Delta \mathrm{P}_{s a t}\right)$ lacking the Rex motif, five additional strains were constructed: 1$)$ a restored promoter sequence with the exact promoter region upstream of sat as the parental strain (restored $\mathrm{P}_{\text {sat }}$ ), 2) a promoter with the -147 conserved " $\mathrm{G}$ " residue altered to an " $\mathrm{A}$ " ( $\mathrm{G}$ -147 A), 3) a promoter with the distal inverted repeat "GTA" altered to "ACG" (-147 to -

145) (IR1), 4) a promoter with the well-conserved proximal inverted repeat "CAC" altered to "TGT" (-135 to -133) (IR2), and 5) a promoter with mutations to both inverted repeat sequences in a single strain (IR1and2).

The mutants along with the restored promoter and parental strain were grown by sulfate respiration and pyruvate fermentation (Figure 3-6). Growth was similar for all strains with the exception of that deleted for the promoter $\left(\Delta \mathrm{P}_{\text {sat }}\right)$, which was unable to grow by sulfate respiration and consistently grew slightly more efficiently by pyruvate fermentation. 


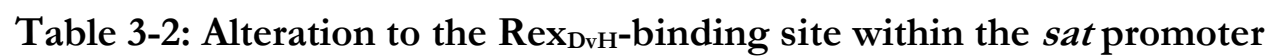

\begin{tabular}{|c|c|c|c|c|c|}
\hline Strain & Name & sat promoter sequence & & $\begin{array}{c}\text { EMSA } \\
\text { Fragment }\end{array}$ & $\begin{array}{c}\text { Estimated } \\
K_{d}(n M)\end{array}$ \\
\hline JW710 & Parental & CGCTTGACATTTTGTAAATTTTTTCACAAGACGGAATCAA... & (120 bps)...ATG & $\mathrm{C}$ & $\sim 100$ \\
\hline JW9293 & $\Delta \mathrm{P}_{\text {sat }}$ & $\operatorname{CGCTTGACAT}\left(\Delta \mathrm{P}_{\text {sat }}-150\right.$ to -1$) \ldots$ & (1573 bp)...ATG & N.A. & N.A. \\
\hline JW9312 & Restored $\mathrm{P}_{\text {sat }}$ & CGCTTGACATTTTGTAAATTTTTTCACAAGACGGAATCAA... & (120 bps)...ATG & N.A. & N.A. \\
\hline JW9314 & $G-147$ A & CGCTTGACATTTTATAAATTTTTTCACAAGACGGAATCAA... & (120 bps)...ATG & $\mathrm{C}^{\mathrm{I}}$ & $\sim 500$ \\
\hline JW9316 & IR1 & CGCTTGACATTTTACGAATTTTTTCACAAGACGGAATCAA... & (120 bps)...ATG & $\mathrm{C}^{\mathrm{II}}$ & $>2000$ \\
\hline JW9318 & IR2 & CGCTTGACATTTTGTAAATTTTTTTGTAAGACGGAATCAA... & (120 bps)...ATG & $\mathrm{C}^{\mathrm{III}}$ & $>2000$ \\
\hline JW9320 & IR1and2 & CGCTTGACATTTTACGAATTTTTTTGTAAGACGGAATCAA... & (120 bps)...ATG & $\mathrm{C}^{\mathrm{IV}}$ & $>2000$ \\
\hline
\end{tabular}

List of the strains and the alterations made to the Rex $\mathrm{DvH}_{\mathrm{D}}$-binding site upstream of sat, with a sequence logo of the predicted Rex $\mathrm{D}_{\mathrm{DvH}}{ }^{-}$ binding site shown above. The Rex ${ }_{D_{v} H}$-binding site is underlined with the alterations made shown in red. Alignment of sequences are shown relative to the assumed translational start codon for sat. Fragments used for EMSA are displayed along with the estimated $\mathrm{K}_{\mathrm{d}}$ $(\mathrm{nM})$ for each 40-bp fragment. N.A. denotes not assessed. Strains: parental (JW710), sat promoter exchange deletion $(\Delta \mathrm{P} s a t)$, restored promoter (Psat), conserved "G-147" altered to "A" (G-147 A), distal inverted repeat "GTA" altered to "ACG” (IR1), proximal inverted repeat "CAC" altered to "TGT" (IR2), and alterations to both inverted repeat sites (IR1 and2). 


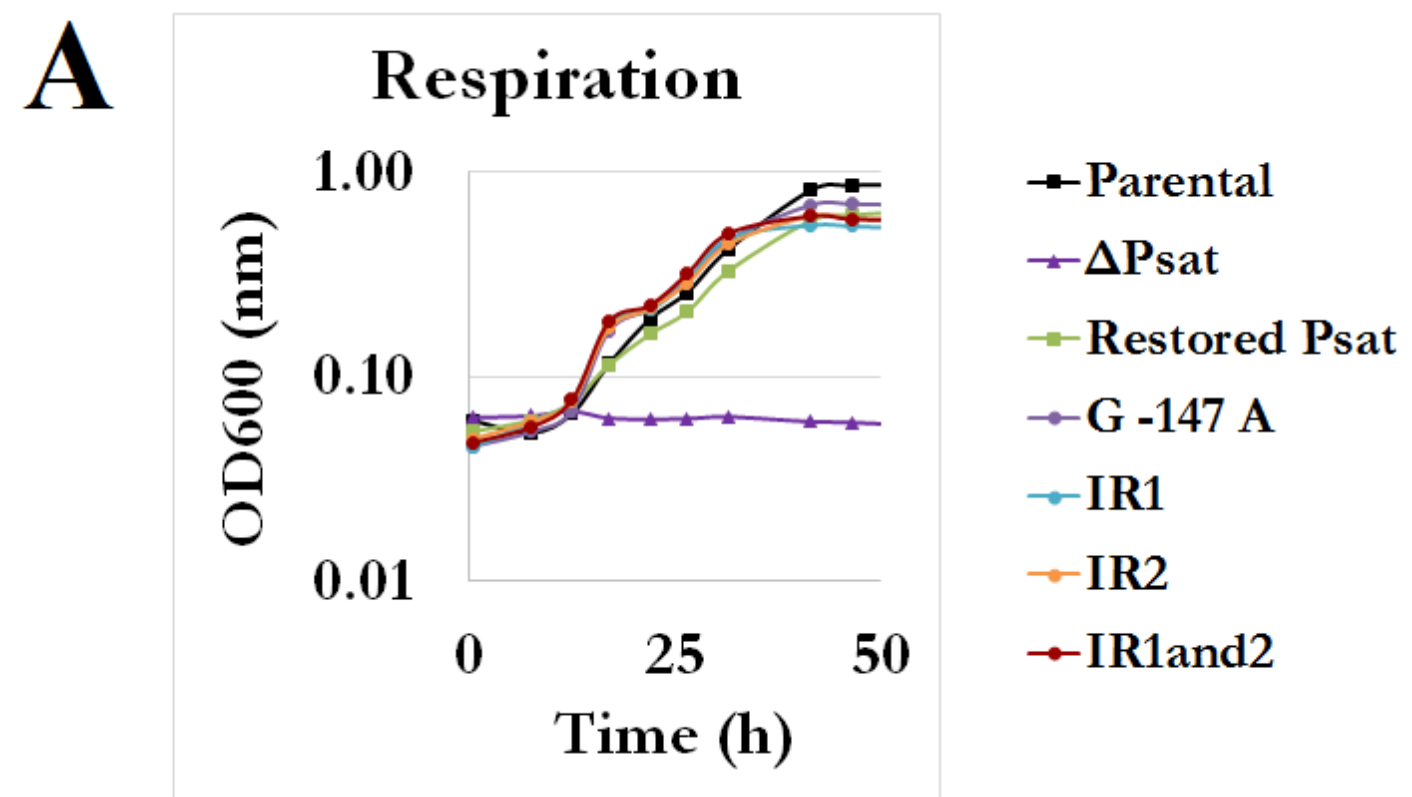

B

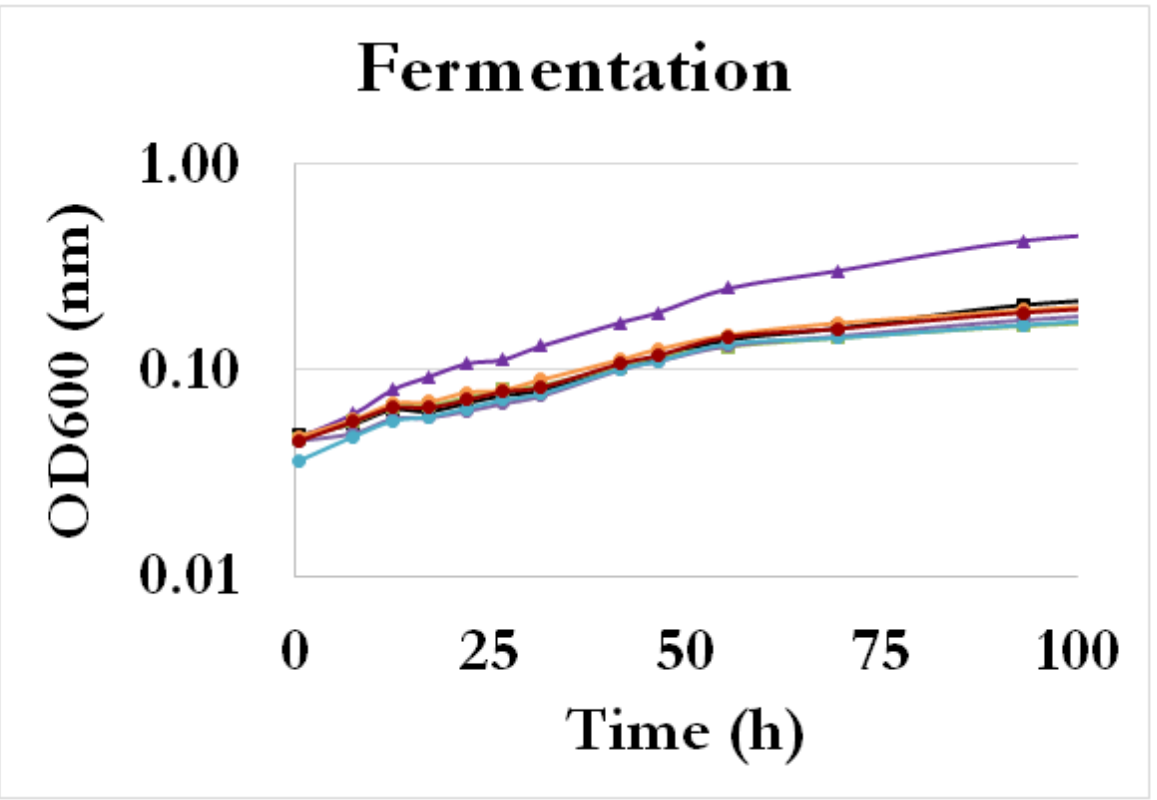

Figure 3-6: Growth kinetics of parental and sat promoter mutants.

Growth of three replicates of mutants and parental strain by A) sulfate respiration or B) pyruvate fermentation. 
It was predicted that the modifications to the $\operatorname{ex}_{\mathrm{DvH}^{-}}$-binding site might prevent

Rex $_{\mathrm{DvH}}$ repression of sat and therefore increase expression of sat. Therefore, samples early in exponential growth were analyzed for sat and rex expression (Table 3-3) (Table A-2). All

strains with sequence changes in the Rex $\operatorname{DvvH}-$ binding site had increased sat expression levels relative to the parental and restored strains.

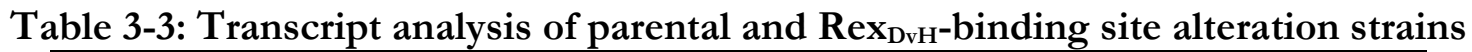

\begin{tabular}{|c|c|c|c|c|}
\hline \multirow[b]{2}{*}{ Strain } & \multirow[b]{2}{*}{ Name } & \multirow[b]{2}{*}{ Media } & \multicolumn{2}{|c|}{ Transcript $^{\mathrm{a}}$} \\
\hline & & & sat & rex \\
\hline JW710 & Parental & MOLS4 & $1.0 \pm 0.1$ & $1.0 \pm 0.1$ \\
\hline JW9293 & $\Delta \mathrm{P}_{\text {sat }}$ & MOLS4 & --- & --- \\
\hline JW9312 & Restored $\mathrm{P}_{\text {sat }}$ & MOLS4 & $0.9 \pm 0.6$ & $0.9 \pm 0.5$ \\
\hline JW9314 & G -147 A & MOLS4 & $2.7 \pm 0.2$ & $1.2 \pm 0.1$ \\
\hline JW9316 & IR1 & MOLS4 & $1.8 \pm 0.1$ & $0.9 \pm 0.1$ \\
\hline JW9318 & IR2 & MOLS4 & $4.0 \pm 0.3$ & $1.0 \pm 0.1$ \\
\hline \multirow[t]{2}{*}{ JW9320 } & IR1and2 & MOLS4 & $4.6 \pm 0.6$ & $1.4 \pm 0.3$ \\
\hline & & & \multicolumn{2}{|c|}{ Transcript $^{\mathrm{b}}$} \\
\hline Strain & Name & Media & sat & rex \\
\hline JW710 & Parental & MOYPyr & $1.0 \pm 0.1$ & $1.0 \pm 0.1$ \\
\hline JW9293 & $\Delta \mathrm{P}_{\text {sat }}$ & MOYPyr & N.D. & $0.6 \pm 0.1$ \\
\hline JW9312 & Restored $\mathrm{P}_{\text {sat }}$ & MOYPyr & $1.0 \pm 0.3$ & $1.6 \pm 0.7$ \\
\hline JW9314 & G -147 A & MOYPyr & $2.9 \pm 0.7$ & $0.9 \pm 0.2$ \\
\hline JW9316 & IR1 & MOYPyr & $3.7 \pm 0.7$ & $0.9 \pm 0.1$ \\
\hline JW9318 & IR2 & MOYPyr & $2.8 \pm 0.5$ & $1.1 \pm 0.1$ \\
\hline JW9320 & IR1and2 & MOYPyr & $2.3 \pm 0.1$ & $1.5 \pm 0.1$ \\
\hline
\end{tabular}

OD600 was monitored through growth and samples were collected for analysis by qRT-PCR at early-exponential. Approximately $100 \mathrm{ng}$ of TURBO ${ }^{\mathrm{TM}}$ DNase-treated RNA was converted to cDNA and $1 \mu \mathrm{L}$ of cDNA (5 ng of RNA) was used per qRT-PCR. Analysis of each gene was conducted separately for each medium tested. MOLS4, efficiency for genes: $r p l S=91.8 \%, r p m C=84.2 \%$, sat $=92.5 \%$, rex $=$ 83.6\%. MOYPyr, efficiency for genes: $r p l S=90.7 \%, r p m C=106.1 \%$, sat $=97.7 \%$, rex $=89.2 \%$. Each gene was assessed individually and normalized to reference genes $r p l S$ and $r p m C$. JW9293 $\left(\Delta \mathrm{P}_{\text {sat }}\right)$ was unable to be grown by sulfate respiration and therefore no data are provided. Error determined as standard error of the mean. ${ }^{a}$ denotes normalized to sample JW710 MOLS4. ${ }^{\text {b }}$ denotes normalized to sample JW710 MOYPyr. N.D. Not Detected. 
dsDNA fragments of $40 \mathrm{bp}$ containing the same alterations that were introduced into the genome were assayed for interactions with increasing Rex $\mathrm{DvH}_{\mathrm{v}}$ concentrations $(0-2000$ nM) (Figure 3-7). For three of the altered fragments tested, G -147 A, IR2 and IR1and2 (C, $\mathrm{C}^{\mathrm{III}}$ and $\mathrm{C}^{\mathrm{IV}}$ ) no shift was observed (dissociation constant, $\mathrm{K}_{\mathrm{d}}>2000 \mathrm{nM}$ ). However, the fragment that contained the three base alteration to the distal inverted repeat sequence, IR1 $\left(\mathrm{C}^{\mathrm{II}}\right)$, did shift $\left(\mathrm{K}_{\mathrm{d}} \sim 500 \mathrm{nM}\right)$ although not to the same extent as the wild-type sequence $\left(\mathrm{K}_{\mathrm{d}} \sim 100 \mathrm{nM}\right)$. This result was rather interesting because this sequence also contained the $\mathrm{G}$ 147 A alteration that appeared to completely disrupt the binding. As expected, no sat transcription was detected for the strain deleted for the sat promoter grown by pyruvate fermentation (Table 3-3). The expression of rex was also examined to verify that an unexpected decrease in $\operatorname{Rex}_{\mathrm{DvH}}$ caused by a transcriptional change was not a factor for the observed differences in sat expression. Across the $\mathrm{P}_{\text {sat }}$ strains, rex expression was not

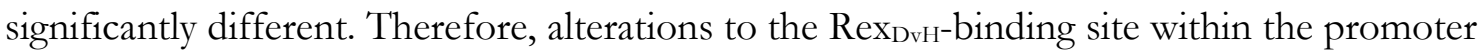
sequence of sat increased sat expression confirming the importance of the highly conserved base pairs in the motif.

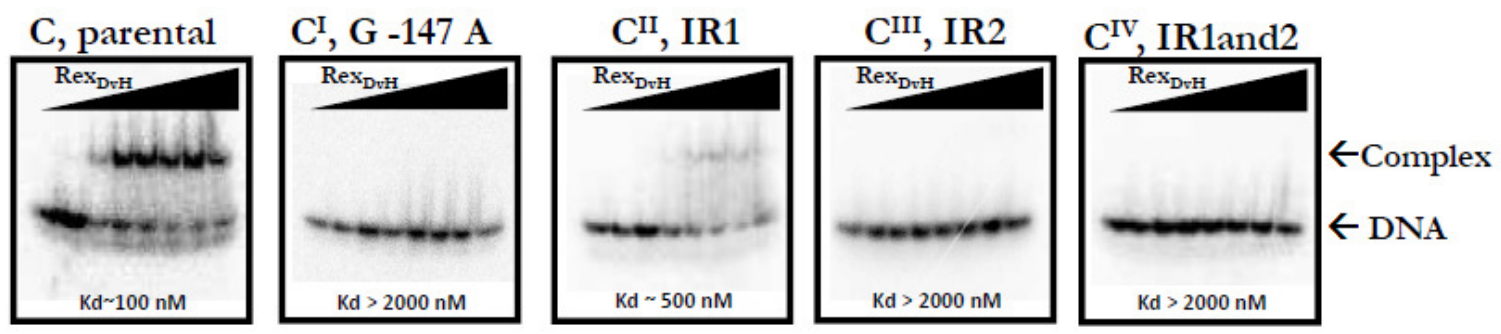

Figure 3-7: Electrophoretic assay demonstrating $\operatorname{Rex}_{\mathrm{DvH}}$ binding to consensus site. Rex $x_{\mathrm{DvH}}$ bound to native (fragment $\mathrm{C}$ ) and altered $\operatorname{Rex}_{\mathrm{DvH}^{-}}$ binding sites $\left(\mathrm{C}^{\mathrm{I}}, \mathrm{C}^{\mathrm{II}}, \mathrm{C}^{\mathrm{III}}, \mathrm{C}^{\mathrm{IV}}\right)$. Rex $\mathrm{x}_{\mathrm{DvH}}$ was added with increasing concentration $(0,10,100,250,500,750,1000$ and $2000 \mathrm{nM})$ to each DNA fragment $(0.1 \mathrm{nM}$, $1 \mathrm{nM}$ prior to column purification). The estimated $\mathrm{K}_{\mathrm{d}}$ is shown. 


\section{Section 3. 8. $\quad \operatorname{Rex}_{\mathrm{DvH}}$ interacts in vitro with $\operatorname{Rex}_{\mathrm{DvH}^{-}}$-binding motif in DvH}

To explore potential Rex $\mathrm{DvH}_{\mathrm{D} H}$ regulation of other target genes, a more high-throughput DNAbinding assay was employed, fluorescent polarization assay (FPA). Twelve operons with putative Rex motifs in their upstream regions, predicted at the time of this study (Ravcheev et al. 2012), were analyzed (Table 3-4). Exact 20-bp predicted Rex $\mathrm{DvH}_{\mathrm{H}}$-binding sites were created with five "G's" at the 5'-end to improve annealing and with four "G's" with a "T" at the 3 '-end to which the fluorophore would be attached. This approach takes advantage of the fact that the degree of polarization of a fluorophore is inversely related to its molecular rotation. Thus the change in fluorescence of a fast moving small unbound DNA fragment compared to a larger $\operatorname{Rex}_{\mathrm{DvH}}$ bound-DNA fragment is evidence of protein-DNA interaction. By increasing the protein concentration over a range of values (0-1000 nM), a dissociation constant was determined for Rex $\mathrm{x}_{\mathrm{DvH}}$ with each 6-FAM ${ }^{\mathrm{TM}}$ labeled dsDNA fragment. Dissociation constants were determined, $\mathrm{K}_{\mathrm{d}} \sim 40-105 \mathrm{nM}$, and were similar to previously published values for Rex, $K_{d} \sim 1-100 \mathrm{nM}$ (Wang et al. 2008; Ravcheev et al. 2012). The two techniques used in this study to calculate protein-DNA interaction between $\operatorname{Rex}_{\mathrm{DvH}}$ and the

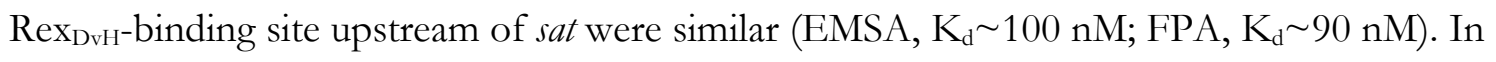
conclusion, Rex $x_{\mathrm{DvH}}$ protein was determined to interact in vitro with all predicted $\mathrm{Rex}_{\mathrm{DvH}^{-}}$ binding sites and the calculated $\mathrm{K}_{\mathrm{d}}$ values were similar. 
Table 3-4: Rex $x_{\mathrm{DvH}}$ interaction with predicted $\operatorname{Rex}_{\mathrm{DvH}}$-binding sites

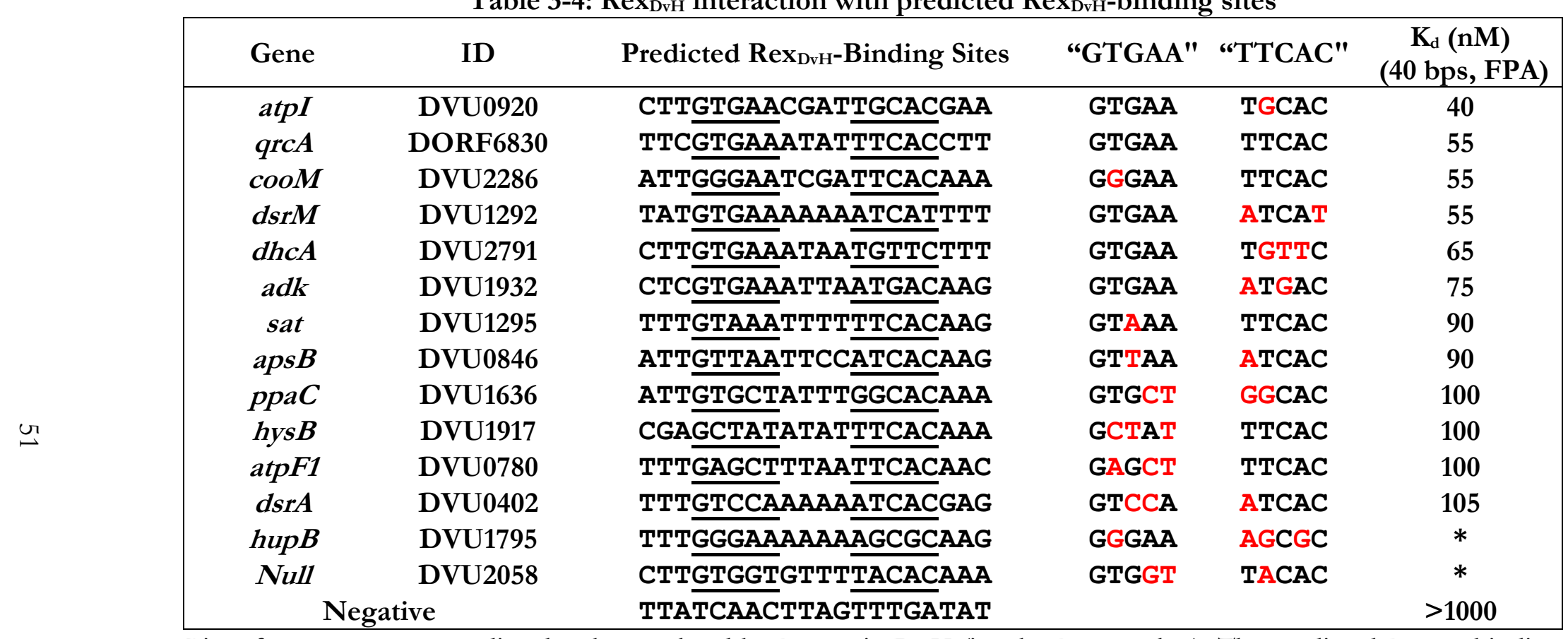

List of target operons predicted to be regulated by $\mathrm{Rex}_{\mathrm{DvH}}$ in DvH (i.e. the Rex regulon). The predicted Rex $\operatorname{DvH}_{\mathrm{Dv}}$-binding sites are aligned across all sequences, with underlined region (inverted repeat) being the minimum sequence for Rex binding. Differences of the half-site from the consensus are marked in red. The calculated dissociation constant $\left(K_{d}\right)$ for Rex $_{\mathrm{DvH}}$ and the predicted Rex $\mathrm{Dvv}_{\mathrm{Du}}$-binding site, calculated by fluorescence polarization assay (FPA), are shown. * FPA was not performed on these sequences. 


\section{Section 3. 9. Discussion}

The sulfate reduction gene sat has been shown to be altered in expression depending on the available electron acceptor (Wall et al. 2008). Bioinformatic predictions for the sulfatereduction pathway, including co-expression studies of the genes as well as looking for conserved motifs upstream of these genes in multiple Desulfovibrio species, proposed regulatory contributions from Rex (encoded by DVU0916) (Ravcheev et al. 2012), HcpR (encoded by DVU2547) (Rodionov et al. 2004), LysX (encoded by DVU2567) and other DNA-binding proteins (encoded by DVU0057, DVU0744, DVU1142, DVU2275, DVU2690, DVU2799, and DVU2802) (Turkarslan et al. 2013). Preliminary examination of several of these potential regulators is discussed in Chapter 5; however, in this chapter, Rex $_{\mathrm{DvH}}$ was characterized for its role in sat expression.

Preliminary transcript studies examining sat expression of a Rex mutant in DvH had shown that the deletion of rex increased sat expression relative to a parental strain (data not shown). These findings are consistent with the role of $\operatorname{Rex}_{\mathrm{DvH}}$ as a repressor of sat. This transcription factor has been proposed to be responsible for redox poise of the cell through the $\mathrm{NADH} / \mathrm{NAD}^{+}$ratio and to alter cellular metabolism to reestablish the pyridine nucleotide balance (Ravcheev et al. 2012). To confirm the role of $\operatorname{Rex}_{\mathrm{DvH}}$, the Rex mutant was cultured in two media proposed to alter the redox status of the cell, and sat transcription was measured (Table 3-1). Examination of the Rex mutant revealed increased sat transcripts compared to the parental strain for both growth modes. Furthermore, the differential increase in transcript levels observed in the absence of sulfate for the parental strain was not maintained in the Rex mutant. We interpreted these observations to mean that $\operatorname{Rex}_{\mathrm{DvH}}$ is a transcriptional repressor for sat responding to redox status in the cell. 
The rex gene encoded on a plasmid under a $n p t$ promoter was introduced into the Rex mutant to test restoration of sat repression. When this complementation construct was respiring sulfate, sat repression was only partially restored. However, rex was transcribed greater than tenfold higher than in the wild type (Table 3-1). Interestingly, for the fermenting culture the expression of sat was restored to wild-type levels. The increased transcription of rex, and therefore we assume the protein $\operatorname{Rex}_{\mathrm{DvH}}$, in the complemented strain might lead to higher than normal levels of repression of other targets of Rex $\mathrm{DvH}_{\mathrm{D}}$ not yet studied. This aberrant expression of rex may adversely affect the overall metabolism of the cell. However, a comparison of growth curves showed little difference in rate or extent of sulfate respiration by the parent, rex deletion and complemented strains under the conditions examined in this study.

Interestingly, there is a conserved hypothetical gene 33 bp downstream of rex, DVU0915, that was originally predicted to be in the same operon. However, the expression of DVU0915 is quite low and from a large battery of transcriptome data publically available for $\mathrm{DvH}$ (http://www.microbesonline.org/), these genes do not appear to be co-regulated. (Figure 3-1). Examination of the intergenic region revealed several strong hairpins that might function as transcriptional regulators consistent with separate operonal structure for the adjacent genes. Alternatively, the deletion of the DNA sequence of rex may have eliminated regulatory elements responsible for the proper expression of DVU0915. Preliminary studies examining DVU0915 expression in the three strains revealed that DVU0915 expression was indeed elevated in the Rex mutant and complemented strain compared with the parental strain (data not shown). Therefore, the increased abundance of DVU0915 may contribute to the inconsistencies in the expression of sat in the complemented strain, although no function is known for the protein encoded by this gene. 
To date, it has been assumed that $\operatorname{Re}_{\mathrm{DvH}}$ blocks transcription by preventing the polymerase from binding to promoter DNA, limiting the expression of the downstream gene. Interestingly, based on the identified TSS for respiring cultures the -10 position should be within the Rex $\mathrm{DvH}_{\mathrm{vH}}$-binding site, specifically overlapping with the proximal inverted repeat (IR2). However, no conventional -10 site could be identified but a site similar to a classical

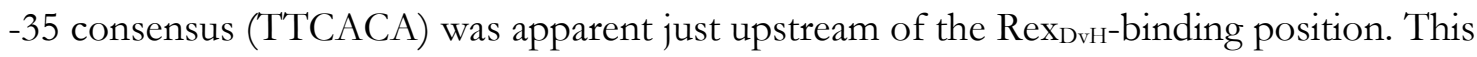
was interpreted to mean that the RNA polymerase binding site of a respiring culture is within the Rex $x_{\mathrm{DvH}}$-binding site and therefore the proposed RNA polymerase occlusion mechanism for Rex seems plausible during respiration.

However, this RNA polymerase occlusion mechanism does not explain the second site of transcript initiation apparently functioning when $\mathrm{DvH}$ is grown by fermentation and even more sat transcript is produced. When sulfate becomes limiting and NADH is plentiful, increased Sat could possibly scavenge low sulfate as a terminal electron acceptor and/or provide reduced sulfur for cell biosynthesis. Preliminary work suggests that the levels of Sat may be linked to sulfate uptake (Zane et al., in prep). Therefore, it would be reasonable, when the preferred electron acceptor is limiting (e.g. sulfate), that the cell might increase the expression of a gene that encodes for a protein that may facilitate sulfate uptake. A second possibility is that there is an alternate regulator that recognizes a specific ligand (e.g. sulfate or sulfite) and the absence of this ligand is the signal for the selection of the second start site of transcription. It would seem plausible that sulfite, which at high concentrations is toxic to $\mathrm{DvH}$, may be a ligand for controlling the overall expression of sat as Sat activates sulfate for reduction to sulfite. This latter interpretation might also explain why sat expression is relatively low when sulfite is the electron acceptor and much higher with sulfate. 
It was predicted that the deletion of rex should resemble the state at which Rex should be removed from the promoter (i.e. fermentation); therefore, when the Rex $\mathrm{x}_{\mathrm{DvH}}$ mutant had the same TSS pattern as the parental strain it was clear that additional components are most certainly involved in addition to Rex, and that the order of addition of these other factors may be the mechanism for regulation and not just the presence or absence of Rex $\operatorname{DvH}_{\text {. }}$ Therefore, the mechanism proposed above, regarding another potential regulator that responds to sulfite levels, seems reasonable. Sulfite and not the protein Rex $\mathrm{x}_{\mathrm{DvH}}$ could be controlling the selection of the transcriptional start site. Overall, sat is still subject to transcriptional control by $\operatorname{Rex}_{\mathrm{DvH}}$ in the fermentative condition since transcription is derepressed to even higher levels when $\operatorname{ex}_{\mathrm{DvH}}$ and pyruvate is being fermented.

Because sat expression was increased significantly as a result of a deletion of rex, we sought to examine the interaction of Rex $\mathrm{x}_{\mathrm{DvH}}$ with the promoter of sat (Table 3-2). To confirm the specific bases required for interaction, in vitro assays were performed on short DNA sequences of the promoter region. (Figure 3-7). All alterations constructed in the consensus binding site disrupted the interaction between $\operatorname{ex}_{\mathrm{DvH}}$ and the DNA in vitro. Interestingly, when a highly conserved base (G-147) was altered to an " $\mathrm{A}$ " there was a complete loss of detectable interaction (fragment C', G-147A, "GTA” altered to "ATA"); however, a triple mutation at IR1 (fragment CI " "GTA" altered to “ACG”), which included the G-147A mutation, caused only a slight decrease in the interaction and not the complete loss that was observed for the single base mutation. Closer examination of the sequence revealed that the modified sequence for fragment $C$ II ("ACG"), for which a " $G$ " is now in the third position, may still resemble the consensus site ("GTG”), but that the single base mutation does not. Therefore, a partially restored binding may occur, but only when the second half site, "CAC", is present as in IR1 (fragment $\mathrm{C}^{\mathrm{II}}$ ). However, additional mutation 
studies would be required to further characterize the specific contribution of each base on Rex ${ }_{\text {DvH }}$ binding.

Strains containing the altered promoter sequences discussed above were then constructed and grown by sulfate respiration or pyruvate fermentation and compared to the parental strain (Figure 3-6). Additionally, a strain that was deleted for 150 bp upstream of sat $\left(\Delta \mathrm{P}_{\text {sat }}\right)$ was also assayed. As expected, sat expression increased for any mutation that limited Rex $_{\text {DvH }}$ binding to the promoter region but was eliminated in the promoter deletion strain (Table 3-3). Interestingly, $\Delta \mathrm{P}_{\text {sat }}$ grew to a higher cell density while fermenting pyruvate. This phenomenon has been observed for strains deleted for genes in the sulfate reduction step of the respiratory pathway in $\mathrm{DvH}$ (e.g. quinone-interacting membrane-bound oxidoreductase, qmo $A B C D$ [data not shown] or a tetraheme cytochrome 'TpIc 3 , cyc $A$ (Keller et al. 2014)). The consistent increase in growth on pyvuate may result from a block in sulfate reduction that prevents flux through the activation step, functioning in the parental strain, that requires two ATP equivalents. Although $0.5 \mathrm{mM}$ cysteine is the sulfur source during our growth experiments, it should be noted that nickel $(2.3 \mu \mathrm{M})$ is added as a trace element with sulfate as a counterion in our preparation.

In a recent study (Ravcheev et al. 2012), $\operatorname{Rex}_{\mathrm{DvH}}$ was predicted to interact with the promoter region for 12 operons, including many that encode proteins functioning in other steps of sulfate reduction in DvH (Table 3-4). Interestingly, most of the targets predicted to be regulated by $\operatorname{Rex}_{\mathrm{DvH}}$ are not involved with NADH oxidation directly (except the Rnf complex) as they are for other bacteria (Ravcheev et al. 2012).

As this study examined only a subset of potential electron donors and acceptors for $\operatorname{Rex}_{\mathrm{DvH}}$ regulation of sat, the effect of other substrates (e.g. pyruvate, formate, $\mathrm{H}_{2}$ or sulfite, thiosulfate) might reveal additional features of Rex regulation. In fact, we recently reported 
that the $\operatorname{Re}_{\mathrm{DvH}}$ mutant was inhibited for growth with thiosulfate as the terminal electron (Korte et al. 2014), an observation that deserves examination. Additionally, Rex $\mathrm{x}_{\mathrm{DvH}}$ has been predicted to regulate over 10 operons containing over 50 genes (Ravcheev et al. 2012). Recently, one of those operons, $d h c A-m f C D G E A B F$, has been shown to decrease in a strain deleted for rex in D. alaskensis G20 (Kuehl et al. 2014), suggesting Rex functions as an activator as well. Finally, over 150 potential regulators have been predicted in $\mathrm{DvH}$, and should be considered either for their interaction with $\operatorname{Rex}_{\mathrm{DvH}}$ or for their role in regulating genes that encode for sulfate reduction. Interestingly, the binding site for $\operatorname{Rex}_{\mathrm{DvH}}$ is similar to several known FNR-family transcription factors that might compete with Rex $\mathrm{x}_{\mathrm{DvH}}$ for binding. Much additional work is needed to identify the transcriptional regulators that signal cellular nutrient and energy status that are integrated at the level of control of sulfate reduction. Several of these additional questions, including the examination of additional targets controlled by $\operatorname{Rex}_{\mathrm{DvH}}$, are the focus of the following chapter. 


\section{Chapter 4: $\quad \operatorname{Rex}_{\mathrm{DvH}}$ alters the electron flow between internal and external reduction processes in $\mathrm{DvH}$}

This chapter is currently in prep, for submission to Applied and Environmental Microbiology.

$\operatorname{Rex}_{\mathrm{DvH}}$ alters the electron flow between internal and external reduction processes in Desulfovibrio vulgaris Hildenborough.

G. A. Christensen ${ }^{1,2}$, S. R. Fels ${ }^{2,3}$, G.M. Zane ${ }^{1,2}$, A.D.Younkin ${ }^{1,2}$, M.M.L. Sousa ${ }^{4,5}$, C.J.

Petzold $^{2,6}$, A.P. Arkin ${ }^{2,6}$, J. D. Wall ${ }^{1,2}$

${ }^{1}$ Department of Biochemistry, University of Missouri, Columbia MO, USA

${ }^{2}$ Ecosystems and Networks Integrated with Genes and Molecular Assemblies, Berkeley CA, USA

${ }^{3}$ Department of Molecular Microbiology and Immunology, University of Missouri, Columbia MO, USA

${ }^{4}$ Department of Cancer Research and Molecular Medicine, Norwegian University of Science and Technology (NTNU), N-7489 Trondheim, Norway

${ }^{5}$ The Proteomics and Metabolomics Core Facility (PROMEC) at NTNU and the Central Norway Regional Health Authority, Trondheim, Norway

${ }^{6}$ Physical Biosciences Division, Lawrence Berkeley National Laboratory, Berkeley, CA, USA 


\section{Section 4.1. Overview}

Rex is a redox sensing protein that regulates the expression of genes that code for proteins responsible for energy conversion. Recently, a Rex regulator was confirmed in Desulfovibrio vulgaris Hildenborough $(\mathrm{DvH})$ through bioinformatic techniques and many previously reported characteristics of Rex were confirmed experimentally in DvH. The work presented here examines the growth characteristics of a Rex mutant of DvH. First, whole-genome gene fitness was determined for a Rex mutant grown on minimal lactate/sulfate medium and compared to fitness data from the parental strain. A small number of genes differed in their contribution to the fitness of the strain deleted for rex. Additionally, growth kinetics of the Rex mutant in defined medium with several substrates compared with the parental strain revealed that the Rex mutant was impaired for growth in thiosulfate-containing medium. A strain deleted for one subunit of an annotated thiosulfate reductase, $\operatorname{phs} A$ was examined to determine its role in the process of thiosulfate reduction and any connection to Rex. A PhsA transposon insertion mutant grew on thiosulfate, suggesting that another thiosulfate reductase may be present. The growth phenotypes of the PhsA and Rex mutants were clearly different. Transcript expression for genes annotated as coding for proteins responsible for sulfate, sulfite, and thiosulfate reduction were determined. The genes that code for proteins responsible for the initial steps of sulfate reduction, sat and aps $A$ were repressed by Rex, but the genes involved in sulfite or thiosulfate reduction ( $d s r A$ and $\operatorname{phs} A$, respectively) were not. Although Rex has been shown to be a transcriptional repressor for sulfate reducing functions, it does not directly repress genes for thiosulfate reduction. Here, a model for Rex in thiosulfate reduction is proposed, suggesting that Rex controls genes responsible for the flow of electrons between the cytoplasm and the periplasm. 


\section{Section 4. 2. Whole-genome gene fitness of a $\operatorname{Rex}_{\mathrm{DvH}}$ mutant grown by lactate with sulfate respiration}

We sought to test for genes that might be changed in importance for growth of a Rex mutant when compared with the parental strain when grown in lactate/sulfate medium. A transposon mutant fitness profiling assay, Transposon L Liquid Enrichment sequencing (TnLE-seq) was performed (Fels et al. 2013; Korte et al. 2014). This procedure generated random transposon mutations within the Rex mutant and parental strain for comparison. To that end, the two strains were grown in MOLS4 medium until the culture reached OD600 0.4 and then deep sequencing was done to determine the abundance and location of each mutation (Figure 4-1) http://desulfovibriomaps.biochem.missouri.edu/fitness/) (Figure 4-2). Gene fitness is shown by $\log _{2} \mathrm{R}$ format, where $\mathrm{R}$ is the ratio of the number of insertions in a gene in the Rex mutant compared to the insertions in that gene in the parental strain. A positive $\mathrm{R}$ correlates to a fitness advantage and a negative $\mathrm{R}$ to a fitness defect. Of the $>3000$ genes within $\mathrm{DvH}, 638$ were considered to be essential with fitness $<-2.2$, while in the Rex mutant 631 genes were considered essential. For both strains, as expected a gene that coded for an enzyme necessary for normal growth (e.g. translation, replication, sulfate reduction) had a high gene-fitness score, as observed by a mutant strain being less abundant in the population. A selected list of individual genes whose fitness was altered greater than twofold in the Rex mutant or were the focus of this study are presented in Table 4-1. The majority of the genes required for sulfate reduction were determined to be essential, including sat, apsBA, qmo $A B C D$, $d s r A B D$ and $d s r C$. Minor fitness differences were observed on both the chromosome and native plasmid (pDV1). Fitness values for genes within operons typically clustered together. 


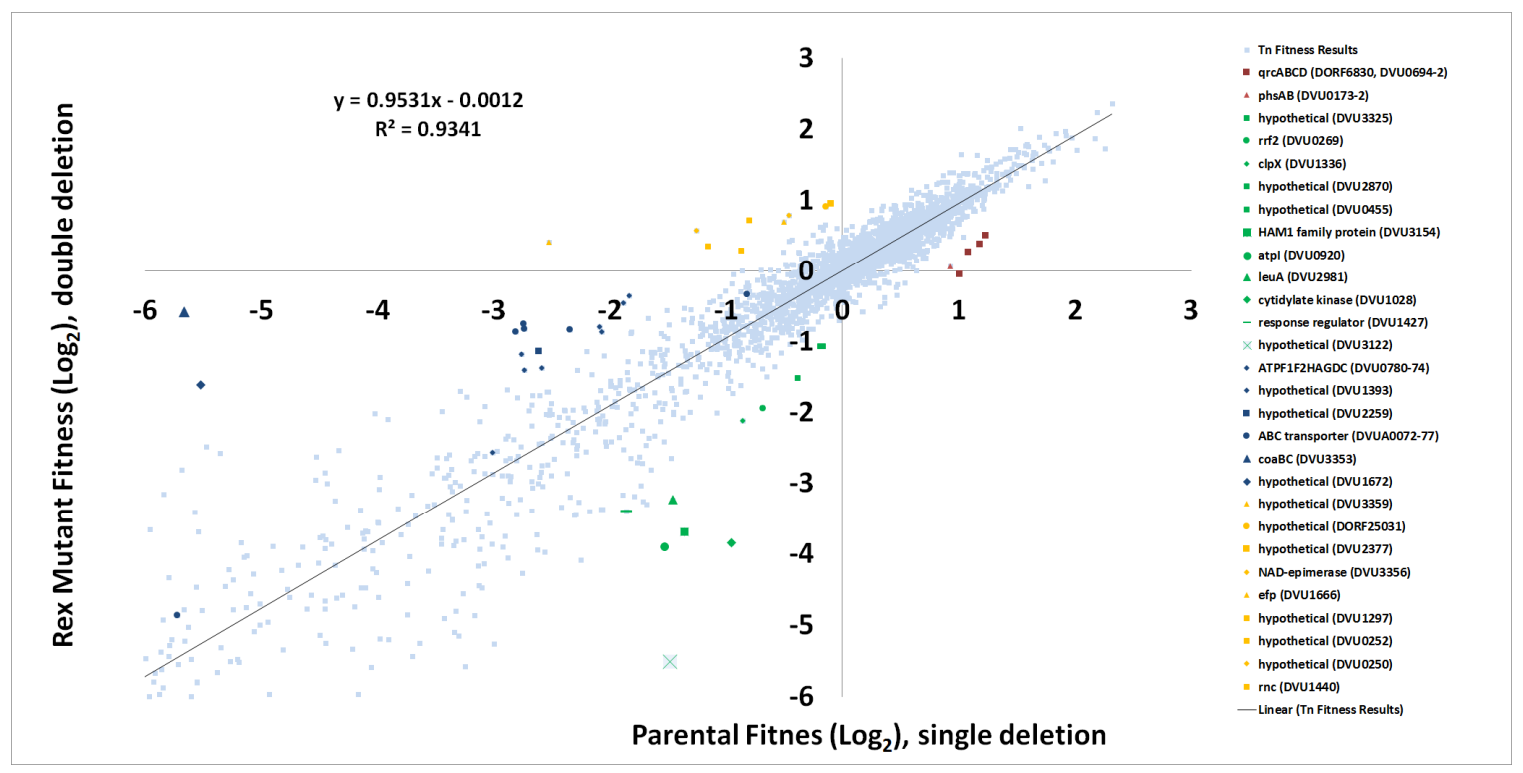

Figure 4-1: Minimal difference in gene fitness between the Rex mutant

and parental strain. Whole-genome gene fitness comparison of parental

DvH (JW710, x-axis) and Rex mutant (JW3319, y-axis) cultures grown in defined lactate respiring medium with sulfate (MOLS4). Data are presented in $\log _{2} \mathrm{R}$ format, where $\mathrm{R}$ is the ratio of the number of insertions in a gene in the Rex mutant compared to the insertions in that gene in the parental strain. A positive $\mathrm{R}$ correlates to a fitness advantage and a negative $\mathrm{R}$ to a fitness defect. Refer to http://desulfovibriomaps.biochem.missouri.edu/mutants/ and Table 4-1 for specific values. Genes different than the linear regression $(y=x)$ are highlighted, color scheme highlights separate quadrants of fitness response between the parental and Rex mutant strains. Red and green (below the line) refer to genes when deleted in the Rex mutant compared to the parental are decreased in fitness (i.e. become more necessary), while yellow and blue (above the line) refer to genes when deleted had improved fitness in the Rex mutant compared with the parental (i.e. become less necessary). 


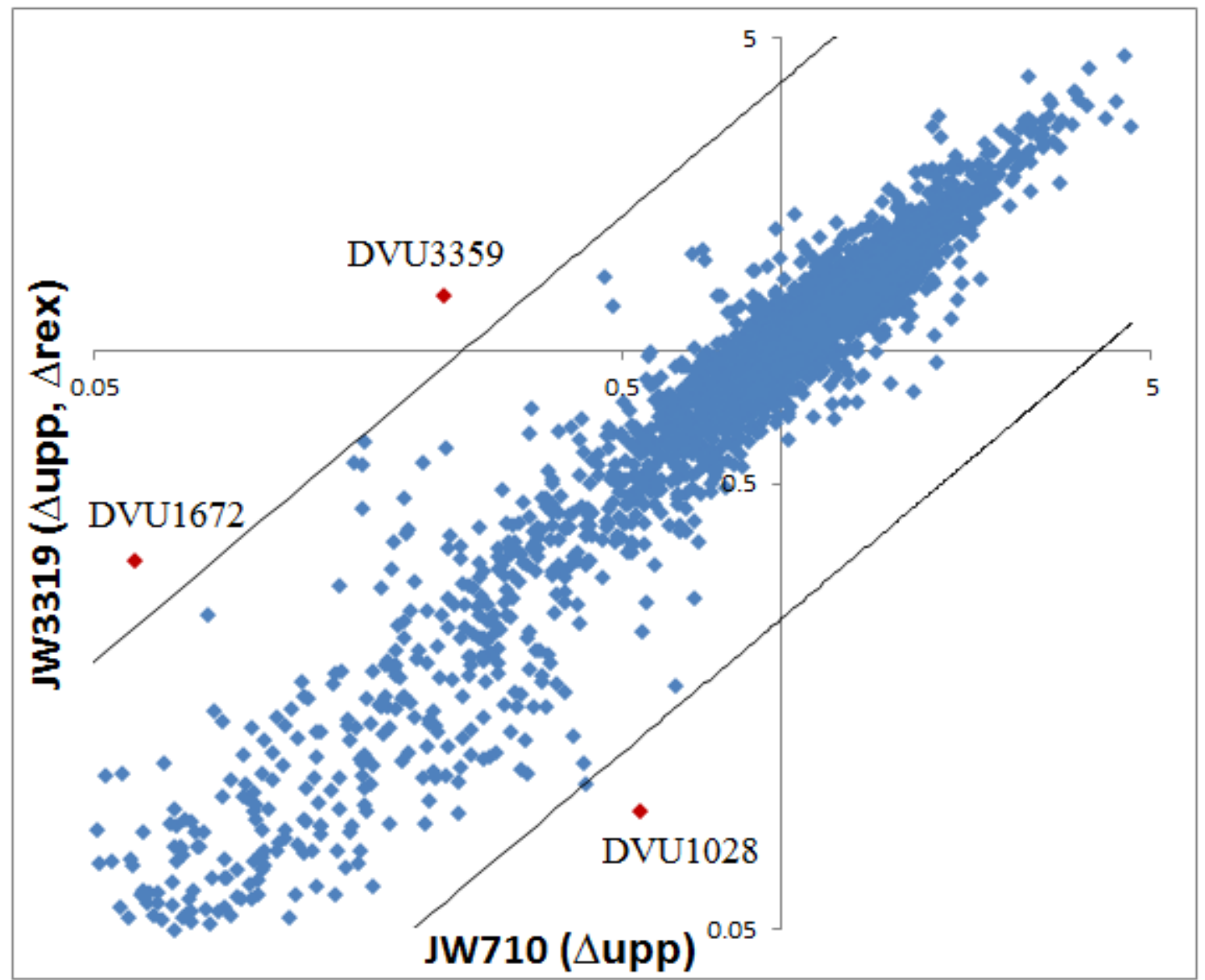

Figure 4-2: Minimal difference in gene fitness. Whole-genome gene fitness comparison of parental DvH (JW710, x-axis) and Rex mutant (JW3319, y-axis) cultures grown in defined lactate respiring medium with sulfate (MOLS4). Axes are shown in standard non-log format. Parallel lines represent $P$ value cutoffs for $P \leq 10^{-5}$. Axes are shown in $\log _{10}$ format. Outliers, in red, are DVU1028, DVU1672 and DVU3359. Image curtousy of Sam Fels. 
Table 4-1: Gene fitness for parental strain and Rex mutant grown by sulfate respiration

\begin{tabular}{|c|c|c|c|c|c|}
\hline \multirow[b]{2}{*}{ DVU\# } & \multirow[b]{2}{*}{ Gene Product Description } & \multirow[b]{2}{*}{$\begin{array}{l}\text { Gene } \\
\text { Name }\end{array}$} & \multicolumn{3}{|c|}{ Fitness $\left(\log _{2}\right)$} \\
\hline & & & Parental & $\begin{array}{c}\text { Rex } \\
\text { mutant }\end{array}$ & $\begin{array}{l}\text { Differ- } \\
\text { ence* }\end{array}$ \\
\hline DVUA00724 & Glycosyl transferase & & -5.7 & -4.9 & 0.8 \\
\hline DVUA0073 & Asparagine synthase & & -2.7 & -0.8 & 1.9 \\
\hline DVUA0074 & Sulfotransferase family protein & & -0.8 & -0.3 & 0.5 \\
\hline DVUA0075 & Radical SAM domain protein & & -2.3 & -0.8 & 1.5 \\
\hline DVUA0076 & ABC transporter, ATP-binding & & -2.7 & -0.8 & 1.9 \\
\hline DVUA0077 & $\mathrm{ABC}$ transporter, permease protein & & -2.8 & -0.9 & 1.9 \\
\hline DVU0774 & ATP synthase, F1 epsilon subunit & $\operatorname{atp} C$ & -3 & -2.6 & 0.4 \\
\hline DVU0775 & ATP synthase, F1 beta subunit & $\operatorname{atpD}$ & -2.8 & -1.2 & 1.6 \\
\hline DVU0776 & ATP synthase, F1 gamma subunit & $\operatorname{atp} G$ & -2.1 & -0.9 & 1.2 \\
\hline DVU0777 & ATP synthase, F1 alpha subunit & $\operatorname{atp} A$ & -1.8 & -0.4 & 1.4 \\
\hline DVU0778 & ATP synthase, F1 delta subunit & $\operatorname{atp} H$ & -2.6 & -1.4 & 1.2 \\
\hline DVU0779 & ATP synthase F0, B subunit & $\operatorname{atp} F 2$ & -2.7 & -1.4 & 1.3 \\
\hline DVU0780 & ATP synthase F0, B subunit & $\operatorname{atp} F 1$ & -1.9 & -0.5 & 1.4 \\
\hline DVU0172 & Thiosulfate reductase & $p h s B$ & 0.9 & 0.1 & -0.8 \\
\hline DVU0173 T & Thiosulfate reductase & phs $A$ & 0.5 & -0.3 & -0.8 \\
\hline DVU0269 & Transcriptional regulator, $\operatorname{Rrf} 2$ & $\operatorname{rrf2}$ & -0.7 & -2.0 & -1.3 \\
\hline DVU0402 & Dissimilatory sulfite reductase & $d s r A$ & -10.8 & -9.6 & 1.2 \\
\hline DVU0403 & Dissimilatory sulfite reductase & $d s r B$ & N.D. & -8.4 & N.A. \\
\hline DVU0404 & Dissimilatory sulfite reductase & $d s r D$ & N.D. & -6.9 & N.A. \\
\hline DVU0531 & High-molecular weight cytochrome c & $b m c F$ & 0.3 & 0.4 & 0.1 \\
\hline DVU0532 & High-molecular weight protein & hmcE & 0.6 & 0.1 & -0.5 \\
\hline DVU0533 & High-molecular weight protein & $h m c D$ & 0.3 & 0.3 & 0.0 \\
\hline DVU0534 & High-molecular weight protein & $b m c C$ & 0.5 & 0.2 & -0.3 \\
\hline DVU0535 & High-molecular weight protein & $b m c B$ & 0.2 & -0.1 & -0.3 \\
\hline DVU0536 & High-molecular weight protein & $b m c A$ & 0.6 & 0.4 & -0.2 \\
\hline DVU06924 & Quinone reductase & $\operatorname{qrcD}$ & 1.0 & -0.1 & -1.1 \\
\hline DVU0693 & Quinone reductase & $\operatorname{qrc} C$ & 1.2 & 0.4 & -0.8 \\
\hline DVU0694 & Quinone reductase & $\operatorname{qrcB}$ & 1.1 & 0.3 & -0.8 \\
\hline DORF6830 & Quinone reductase & $\operatorname{qrc} A$ & 1.2 & 0.5 & -0.7 \\
\hline DVU0846 & Adenylyl-sulfate reductase, beta subunit & $a p s B$ & N.D. & -7.4 & N.A. \\
\hline DVU0847 & Adenylyl-sulfate reductase, alpha subunit & $a p s A$ & -7.4 & -8.2 & -0.8 \\
\hline DVU0848 & $\begin{array}{l}\text { Quinone-interacting membrane-bound } \\
\text { oxidoreductase }\end{array}$ & $q m o A$ & -9.8 & -7.6 & 1.6 \\
\hline DVU0849 & $\begin{array}{l}\text { Quinone-interacting membrane-bound } \\
\text { oxidoreductase }\end{array}$ & $q m o B$ & -8.5 & -7.1 & 1.4 \\
\hline DVU0850 & $\begin{array}{l}\text { Quinone-interacting membrane-bound } \\
\text { oxidoreductase }\end{array}$ & $q m o C$ & -7.7 & -7.7 & 0.0 \\
\hline DVU0851 & Hypothetical protein & $q m o D$ & -4.2 & -9.0 & -4.8 \\
\hline
\end{tabular}




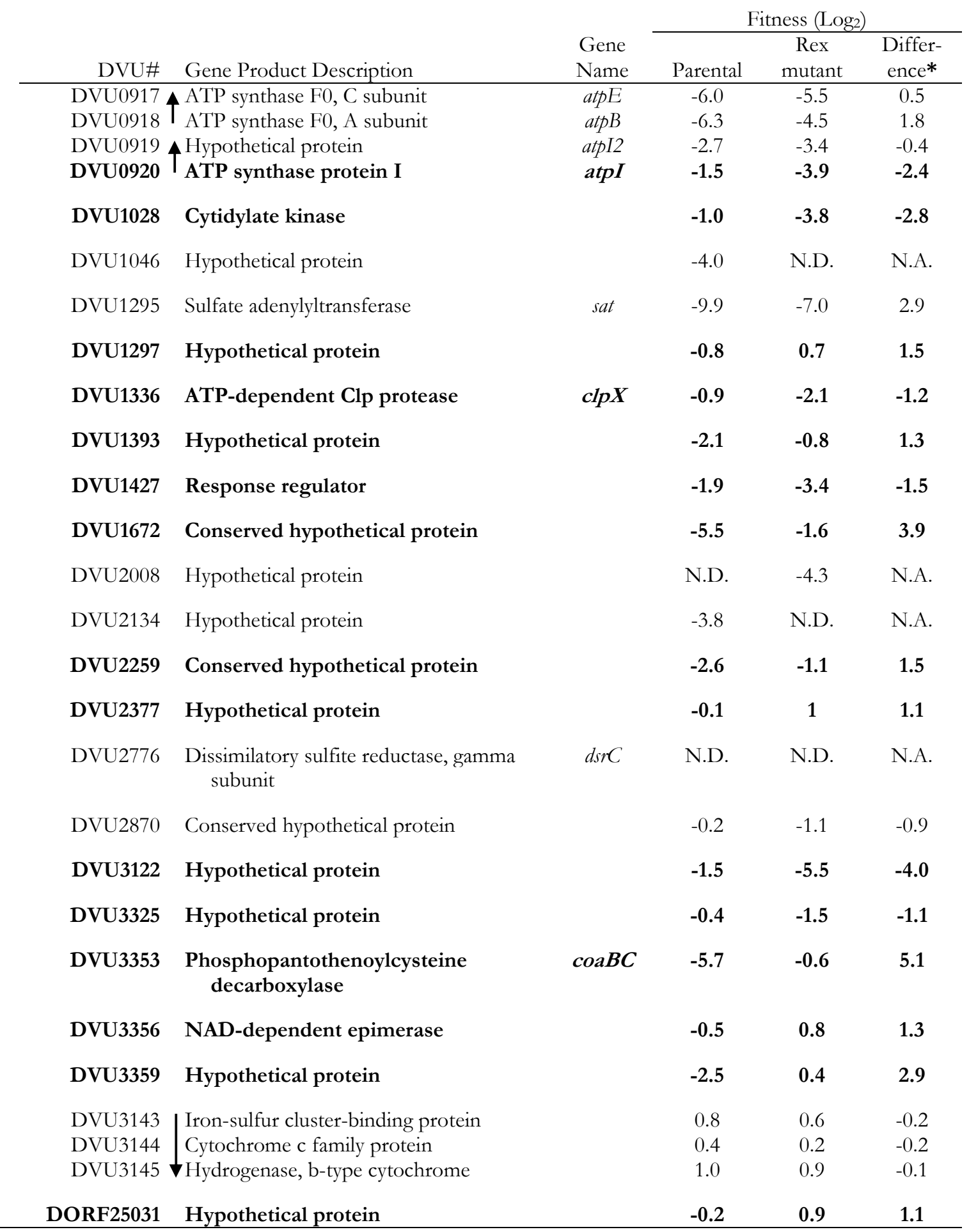

N.D. not detected. N.A. Not applicable. Arrow indicates orientation of operon. Gene fitness is shown by $\log _{2} R$ format, where $\mathrm{R}$ is the ratio of the number of insertions in a gene in the Rex mutant compared to the insertions in that gene in the parental strain. A positive $R$ correlates to a fitness advantage and a negative $\mathrm{R}$ to a fitness defect. *For a gene to be considered different between the Rex mutant and parental strain a fitness difference greater than twofold was required and the gene must be defined as non-essential $\left(\log _{2} R>2.0\right)$. 
Many of the fitness differences observed for genes between the two strains included hypotheticals (Table 4-1). However, genes that were annotated and had decreased fitness (were more important for growth and/or survival) in the Rex mutant compared to the parental strain included: the quinone reductase that is essential for the transfer of electrons from the periplasm to the cytoplasm to drive sulfate reduction ( $q r A B D$, DORF6830DVU0694-2); a putative transcriptional regulator (rrf2, DVU0269) thought to play a role in redox signaling; a response regulator (cheY-like, DVU1427); an ATP-dependent Clp protease (clpX, DVU1336) responsible for protein degradation; and cytidylate kinase (cmk, DVU1028). Those genes that had increased fitness in the Rex mutant compared to the parental strain when deleted included: a putative ABC transporter (DVUA0072-77); the ATP synthase (atpF1F2HAGDC, DVU0780-774); a phosphopantothenoylcysteine decarboxylase (coaBC, DVU3353), and a NAD-dependent epimerase (DVU3356). However, most of these still have a negative fitness-value. The fitness values for genes predicted to be controlled by Rex are shown in Table 4-2. Only two operons, atpF1F2HAGDC and $\operatorname{qr} A B C D$, when deleted in the Rex mutant appeared to increase and decrease the fitness, respectively. 
Table 4-2: Gene fitness for predicted Rex $\operatorname{Dv}_{\mathrm{Dv}}$ regulon

\begin{tabular}{|c|c|c|c|c|c|}
\hline \multirow[b]{2}{*}{ DVU\# } & \multirow[b]{2}{*}{ Gene Product Description } & \multirow[b]{2}{*}{$\begin{array}{l}\text { Gene } \\
\text { Name }\end{array}$} & \multicolumn{3}{|c|}{ Fitness $\left(\log _{2}\right)$} \\
\hline & & & Parental & $\begin{array}{c}\text { Rex } \\
\text { Mutant }\end{array}$ & Difference \\
\hline DVU0402 & Dissimilatory sulfite reductase & $d s r A$ & -10.8 & -9.6 & 1.2 \\
\hline DVU0403 & Dissimilatory sulfite reductase & $d s r B$ & N.D. & -8.4 & N.A. \\
\hline DVU0404 & Dissimilatory sulfite reductase & $d s r D$ & N.D. & -6.9 & N.A. \\
\hline DVU0692个 & Quinone reductase & $q r c D$ & 1.0 & 0.0 & -1.0 \\
\hline DVU0693 & Quinone reductase & $\operatorname{qrc} C$ & 1.2 & 0.4 & -0.8 \\
\hline DVU0694 & Quinone reductase & $\operatorname{qrcB}$ & 1.1 & 0.3 & -0.8 \\
\hline DORF6830 & Quinone reductase & $\operatorname{qrc} A$ & 1.2 & 0.5 & -0.7 \\
\hline DVU0774 $\boldsymbol{4}$ & ATP synthase, F1 epsilon subunit & $\operatorname{atp} C$ & -3.0 & -2.6 & 0.4 \\
\hline DVU0775 & ATP synthase, F1 beta subunit & $\operatorname{atpD}$ & -2.8 & -1.2 & 1.6 \\
\hline DVU0776 & ATP synthase, F1 gamma subunit & $\operatorname{atp} G$ & -2.1 & -0.9 & 1.1 \\
\hline DVU0777 & ATP synthase, F1 alpha subunit & $\operatorname{atp} A$ & -1.8 & -0.4 & 1.4 \\
\hline DVU0778 & ATP synthase, F1 delta subunit & $\operatorname{atp} H$ & -2.6 & -1.4 & 1.2 \\
\hline DVU0779 & ATP synthase F0, B subunit & $\operatorname{atpF2}$ & -2.7 & -1.4 & 1.3 \\
\hline DVU0780 & ATP synthase F0, B subunit & $\operatorname{atpF1}$ & -1.9 & -0.5 & 1.4 \\
\hline DVU0846 & Adenylyl-sulfate reductase & $a p s B$ & N.D. & -7.4 & N.A. \\
\hline DVU0847 $\downarrow$ & Adenylyl-sulfate reductase & $\operatorname{aps} A$ & -7.4 & -8.2 & -0.8 \\
\hline DVU0848 & $\begin{array}{l}\text { Quinone-interacting membrane-bound } \\
\text { oxidoreductase }\end{array}$ & $q m o A$ & -9.8 & -7.6 & 1.6 \\
\hline DVU0849 & $\begin{array}{l}\text { Quinone-interacting membrane-bound } \\
\text { oxidoreductase }\end{array}$ & $q m o B$ & -8.5 & -7.1 & 1.4 \\
\hline DVU0850 & $\begin{array}{l}\text { Quinone-interacting membrane-bound } \\
\text { oxidoreductase }\end{array}$ & $q m o C$ & -7.7 & -7.7 & 0.0 \\
\hline DVU0851 & Hypothetical protein & $q m o D$ & -4.2 & -9.0 & -4.8 \\
\hline \#DVU0916 & AT-rich DNA-binding & rex & -5.3 & -6.5 & -1.2 \\
\hline DVU0917 & ATP synthase F0, C subunit & atpE & -6.0 & -5.5 & 0.5 \\
\hline DVU0918 T & ATP synthase F0, A subunit & $\operatorname{atp} B$ & -6.3 & -4.5 & 1.8 \\
\hline DVU09194 & Hypothetical protein & atpI2 & -2.7 & -3.4 & -0.4 \\
\hline DVU0920T & ATP synthase protein I & atpI & -1.5 & -3.9 & -2.4 \\
\hline DVU1286 & Integral membrane protein & $d s r P$ & -11.0 & -7.4 & 3.6 \\
\hline DVU1287 & Periplasmic (Tat), binds $2[4 \mathrm{Fe}-4 \mathrm{~S}]$ & $d s r O$ & -9.9 & -6.2 & 3.7 \\
\hline DVU1288 & Periplasmic (Sec) triheme cytochrome c & $d s r J$ & -5.7 & -5.2 & 0.5 \\
\hline DVU1289 & Cytoplasmic, binds 2 [4Fe-4S] & $d s r K$ & -7.3 & -7.0 & 0.3 \\
\hline DVU1290 & Inner membrane protein binds 2 heme $b$ & $d s r M$ & -8.8 & -9.6 & -0.8 \\
\hline DVU1291 & Hypothetical protein & & -5.2 & -4.4 & 0.8 \\
\hline DVU1292 & Hypothetical protein & & -1.4 & -1.5 & -0.1 \\
\hline DVU1295 & Sulfate adenylyltransferase & sat & -9.9 & -7.0 & 2.9 \\
\hline DVU1636 & Inorganic pyrophosphatase & $p p a C$ & -7.8 & -4.8 & 3.0 \\
\hline DVU1795 & DNA-binding protein $\mathrm{HU}$ & bup-3 & -0.7 & 0.0 & 0.7 \\
\hline DVU1917 & Periplasmic [NiFeSe] hydrogenase & bys $B$ & -0.2 & 0.0 & 0.2 \\
\hline DVU1918 & Periplasmic $[\mathrm{NiFeSe}]$ hydrogenase & bys $A$ & -0.3 & -0.1 & 0.2 \\
\hline DVU1932 & Adenylate kinase & $a d k$ & -9.2 & -7.0 & 2.2 \\
\hline DVU2058 & HDIG domain protein & null & 0.5 & 0.5 & 0.0 \\
\hline
\end{tabular}


Fitness $\left(\log _{2}\right)$

\begin{tabular}{|c|c|c|c|c|c|}
\hline \multirow[b]{2}{*}{ DVU\# } & \multirow[b]{2}{*}{ Gene Product Description } & \multirow[b]{2}{*}{$\begin{array}{l}\text { Gene } \\
\text { Name }\end{array}$} & \\
\hline & & & Parental & $\begin{array}{c}\text { Rex } \\
\text { Mutant }\end{array}$ & Difference \\
\hline DVU2059 & $\begin{array}{l}\text { Glycosyl transferase, group } 2 \text { family } \\
\text { protein }\end{array}$ & & 0.6 & 0.1 & -0.5 \\
\hline DVU2286 & Hydrogenase & $\operatorname{coo} M$ & -2.4 & -2.0 & 0.4 \\
\hline DVU2287 & Hydrogenase & $\operatorname{cooK}$ & -3.0 & -2.5 & 0.5 \\
\hline DVU2288 & Hydrogenase & $\operatorname{cooL}$ & -3.4 & -3.5 & -0.1 \\
\hline DVU2289 & Hydrogenase & $\operatorname{coo} X$ & -2.7 & -2.2 & 0.5 \\
\hline DVU2290 & Hydrogenase & $\operatorname{coo} U$ & -2.9 & -2.9 & 0.0 \\
\hline DVU2291 & Carbon monoxide-induced hydrogenase & $\mathrm{cooH}$ & -3.4 & -2.9 & 0.5 \\
\hline DVU2292 & $\begin{array}{l}\text { Hydrogenase nickel insertion protein } \\
\text { НypА }\end{array}$ & bур $A$ & -1.8 & -1.7 & 0.1 \\
\hline DVU2293 & Iron-sulfur protein & $\mathrm{cooF}$ & -2.9 & -2.6 & 0.3 \\
\hline DVU2791 & Decaheme cytochrome c & $d h c A$ & 0.5 & 0.6 & 0.1 \\
\hline DVU2792 & NADH:quinone oxidoreductase & $r n f C$ & 0.4 & 0.5 & 0.1 \\
\hline DVU2793 & Electron transport complex protein & $r m f D$ & 0.5 & 0.5 & 0.0 \\
\hline DVU2794 & NADH:quinone oxidoreductase & $\operatorname{rnf} G$ & 0.5 & 0.7 & 0.2 \\
\hline DVU2795 & NADH:quinone oxidoreductase & rnfE & 0.3 & 0.6 & 0.3 \\
\hline DVU2796 & NADH:quinone oxidoreductase & $\operatorname{rnf} A$ & 0.6 & 0.8 & 0.2 \\
\hline DVU2797 & NADH:quinone oxidoreductase & $\operatorname{rnfB}$ & 0.7 & 0.8 & 0.1 \\
\hline DVU2798 & ApbE family protein & & 0.4 & 0.5 & 0.1 \\
\hline
\end{tabular}

Rex regulon predictions from (Novichkov et al. 2012; Ravcheev et al. 2012; Christensen et al. 2014). N.D. not detected. N.A. Not applicable. \#The presence of signal in rex in the Rex mutant is due to "bar-code bleed" (Kircher et al. 2010). Arrow indicates orientation of operon. *For a gene to be considered different between the Rex mutant and parental strain a fitness difference greater than twofold was required and the gene must be defined as nonessential $\left(\log _{2} \mathrm{R}>-2.0\right)$. 


\section{Section 4. 3. The Rex mutant is impaired for growth with thiosulfate as the sole electron acceptor}

A strain deleted for the rex gene has been shown to be minimally affected in growth when cultured by sulfate respiration with lactate in MOLS4 or by pyruvate fermentation in MOYPyr compared to the parental strain (Christensen et al. 2014). Similarly, the gene fitness profile for the Rex mutant while respiring sulfate with lactate was not significantly different from that of the parental strain. However, it was reported that the deletion of rex impairs growth with thiosulfate as the sole electron acceptor (Korte et al. 2014). To further characterize the role of rex, growth rates and extents of the Rex marker-less deletion mutant were determined on various substrates and compared to its parental strain (Figure 4-3) (Table 4-3). The cells were grown by respiration with lactate or pyruvate as the electron donor and sulfate, sulfite, or thiosulfate as the electron acceptor or by pyruvate fermentation. Cells grown by fermentation were expected to differ in their $\mathrm{NADH} / \mathrm{NAD}^{+}$ratio compared with those grown by respiration (Williamson et al. 1967).

Figure 4-3: Deletion of rex, and to an extent $p h s A$, impair growth in thiosulfate. Two separate growth studies of (LEFT) parental DvH (JW710, black) and Rex mutant (JW3319, purple) or (RIGHT) wild-type DvH (DvH, black) and PhsA transposon insertion mutant (GZ8929, yellow) by lactate (60 $\mathrm{mM})$ or pyruvate $(60 \mathrm{mM})$ respiration with sulfate $\left(\mathrm{SO}_{4}{ }^{2-}, 30 \mathrm{mM}\right)$, sulfite $\left(\mathrm{SO}_{3}{ }^{2-}\right.$ , $40 \mathrm{mM}$ ) or thiosulfate $\left(\mathrm{S}_{2} \mathrm{O}_{3}{ }^{2-}, 30 \mathrm{mM}\right.$ ); or pyruvate fermentation (Pyr, 60 $\mathrm{mM}$ ) supplemented with $0.1 \%$ (wt/vol) yeast extract and $0.5 \mathrm{mM}$ cysteine. Error bars represent standard deviation among biological replicates $(n=3)$. 


\section{Rex Mutant}
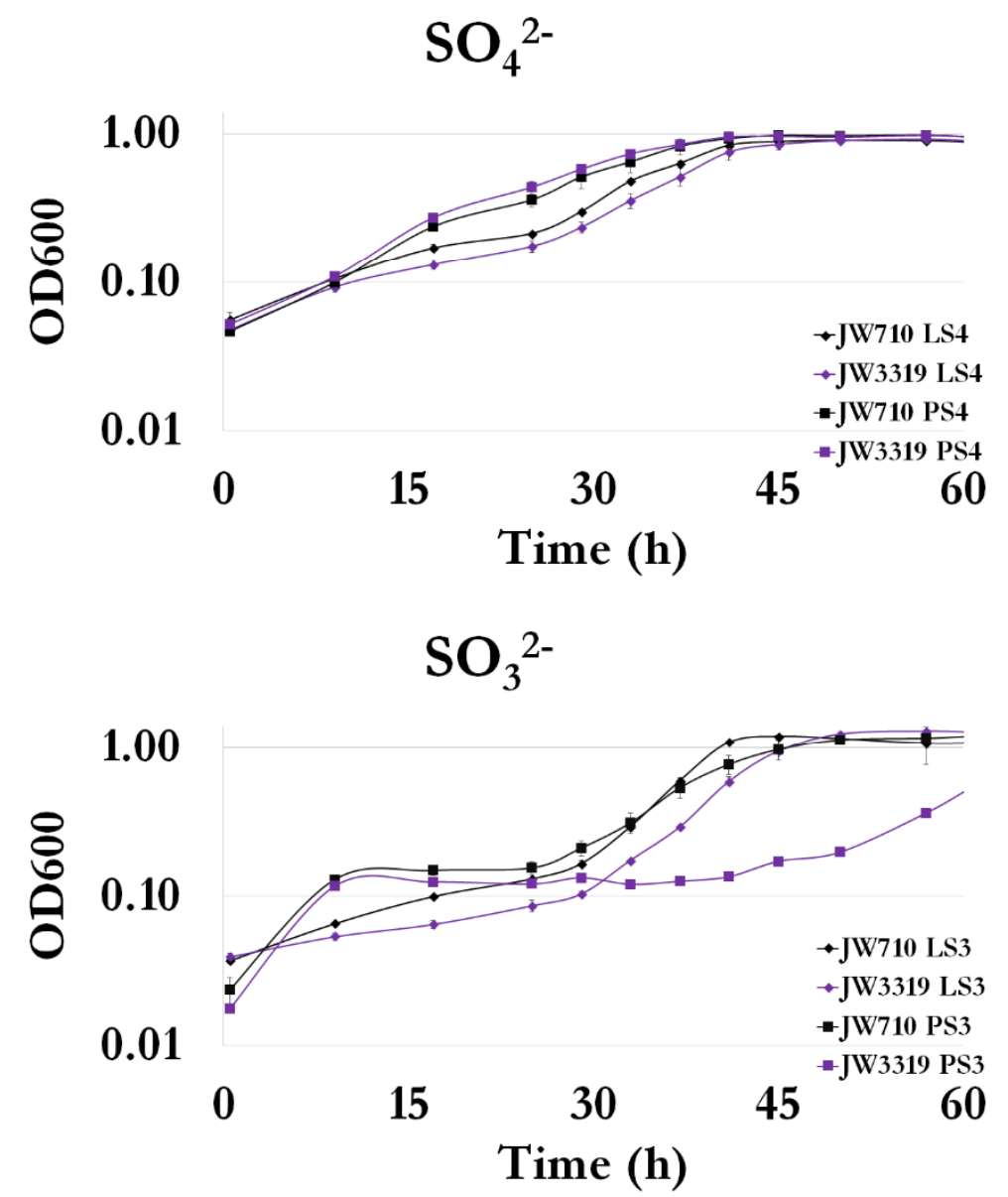

\section{PhsA Mutant}
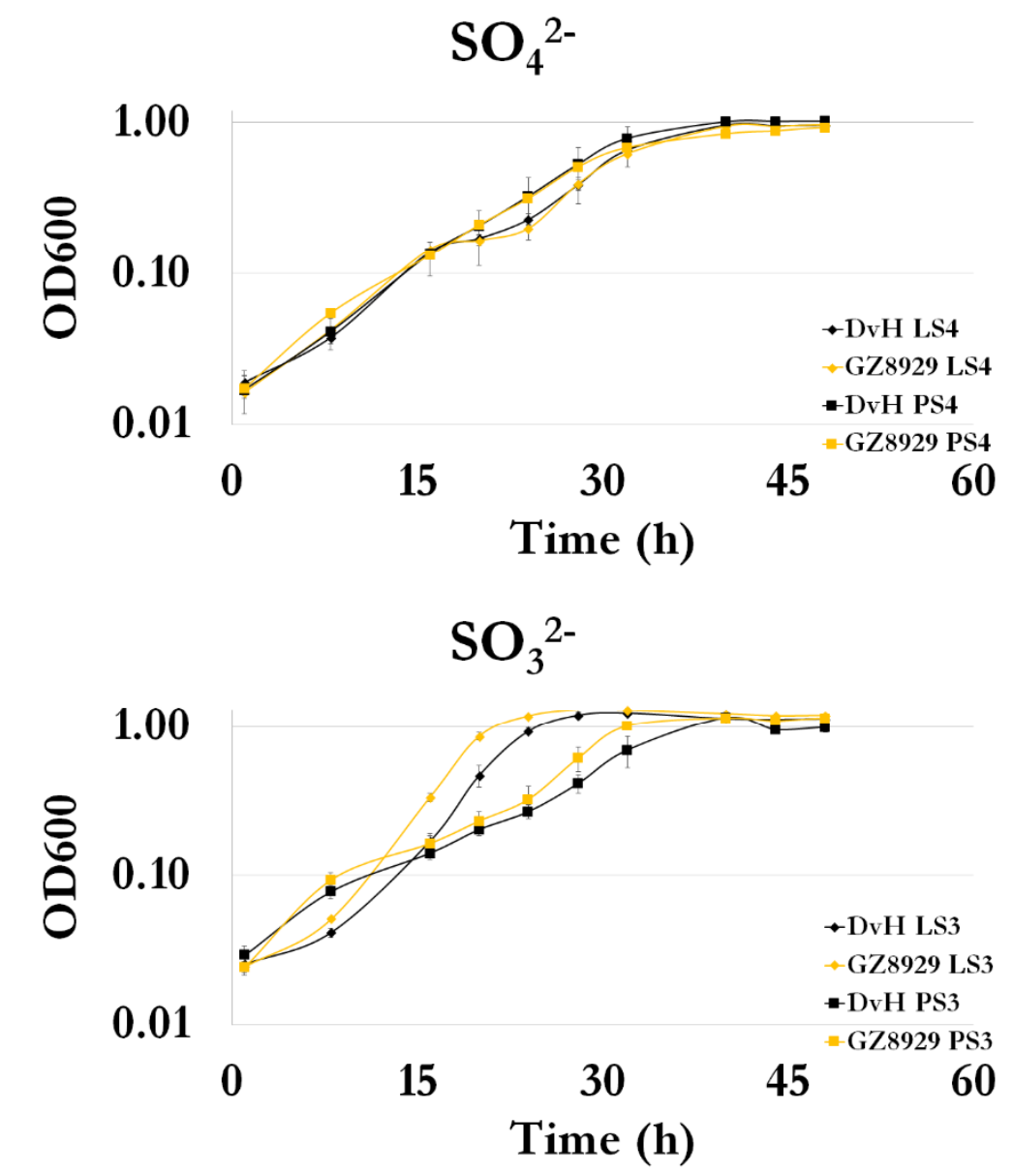


\section{Rex Mutant}
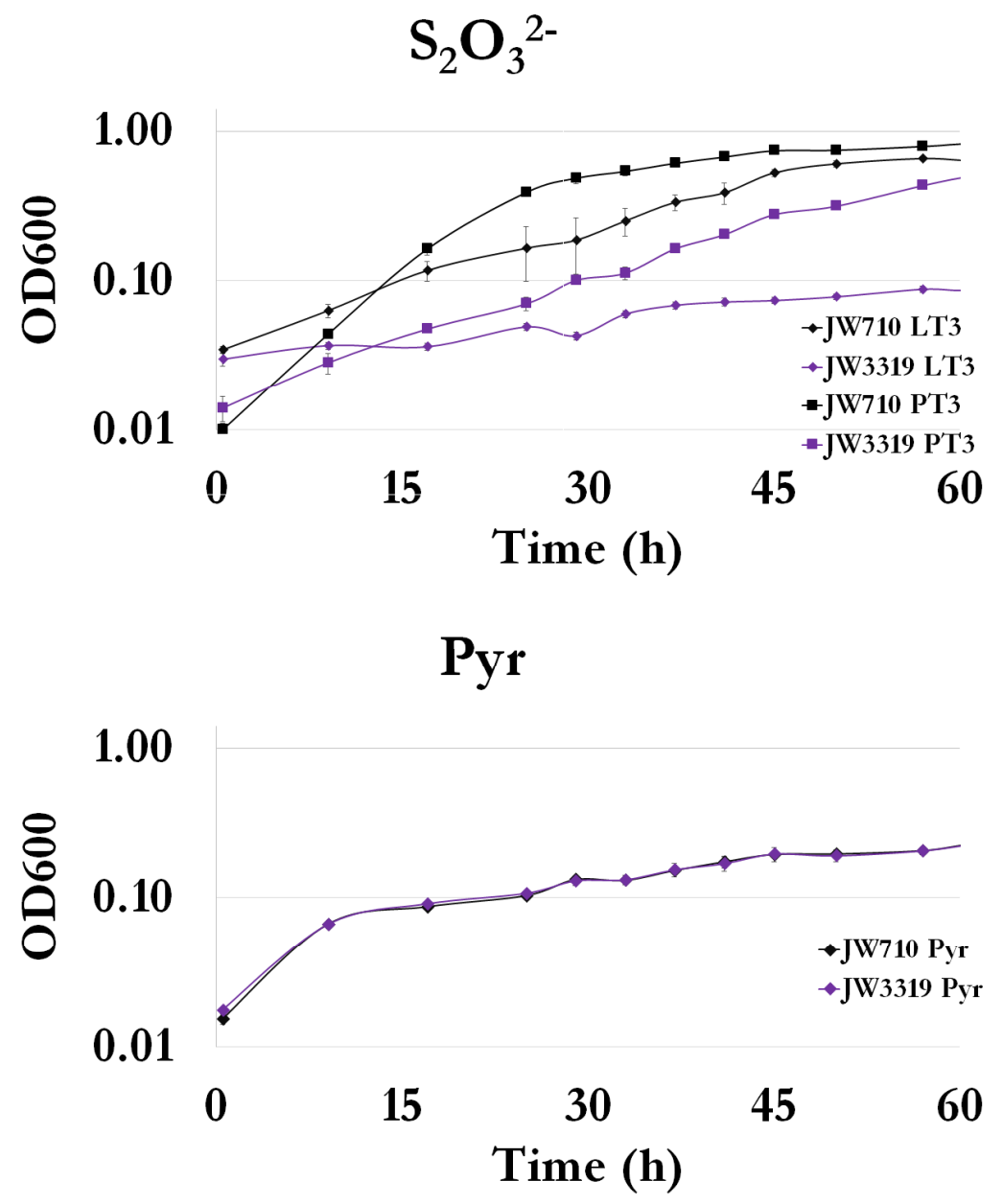

\section{PhsA Mutant}
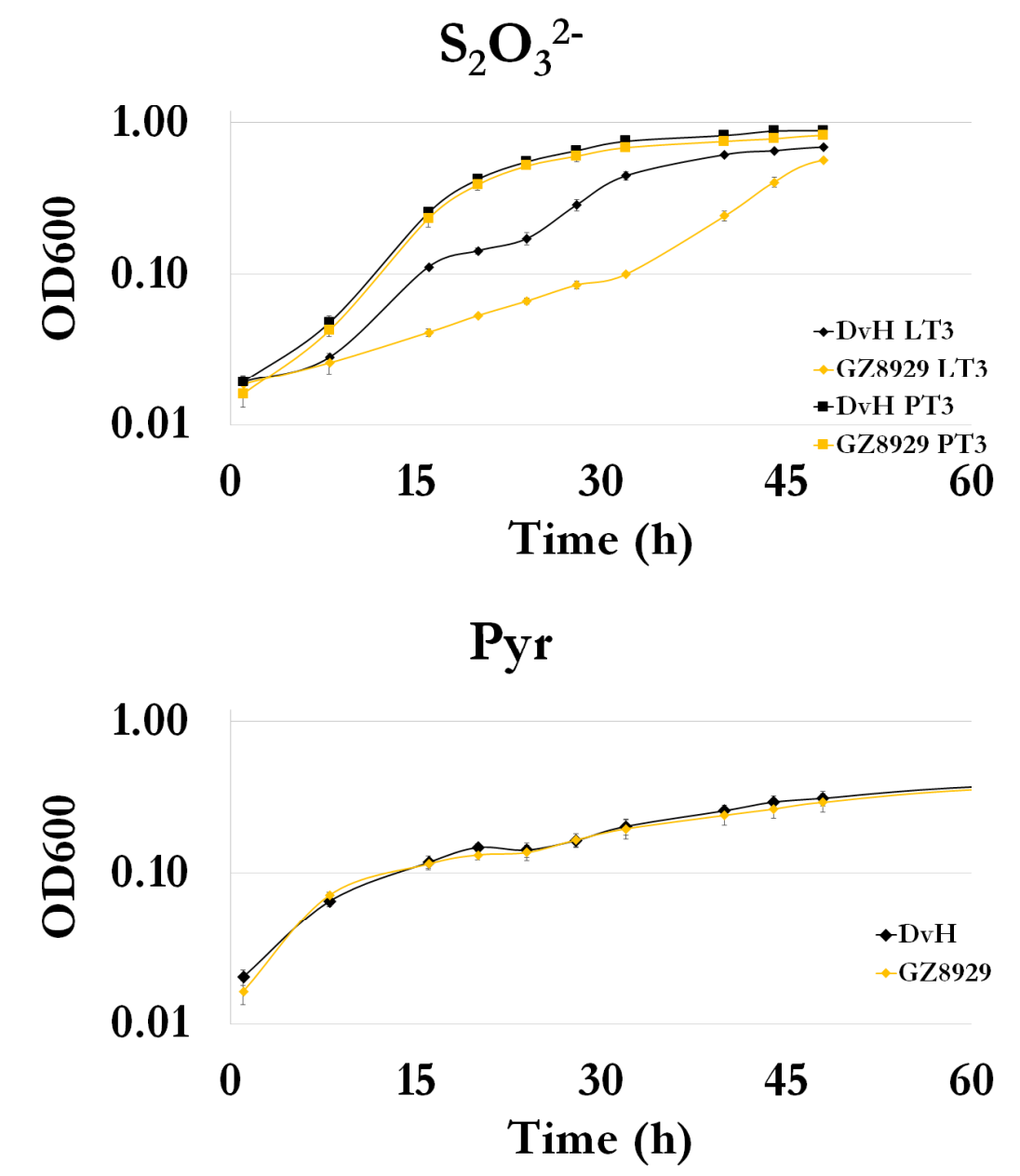
Table 4-3: Sample collection and growth kinetics

\begin{tabular}{|c|c|c|c|c|c|c|}
\hline \multirow[b]{2}{*}{ Name } & \multirow[b]{2}{*}{ Media } & \multicolumn{3}{|c|}{ Growth kinetics ${ }^{\mathrm{a}}$} & \multicolumn{2}{|c|}{ Total Protein ${ }^{\mathrm{b}}$} \\
\hline & & $\begin{array}{c}\text { OD600 } \\
\text { collected }^{\mathrm{c}}\end{array}$ & $\begin{array}{c}\text { Doubling } \\
\text { time }(\mathrm{h})\end{array}$ & OD $600_{\max }$ & $\mu \mathrm{g} / \mathrm{ml}$ & $\begin{array}{l}\mu \mathrm{g} / \mathrm{ml} / \\
\mathrm{OD} 600\end{array}$ \\
\hline JW710 & MOLS4 & $0.29 \pm 0.05$ & $7.1 \pm 0.2$ & $0.91 \pm 0.00$ & $218 \pm 21$ & $240 \pm 22$ \\
\hline Rex mutant & MOLS4 & $0.24 \pm 0.04$ & $8.1 \pm 0.5$ & $0.93 \pm 0.00$ & $203 \pm 12$ & $227 \pm 17$ \\
\hline JW710 & MOLS3 & $0.26 \pm 0.04$ & $4.2 \pm 0.4$ & $1.20 \pm 0.03$ & $294 \pm 12$ & $272 \pm 10$ \\
\hline Rex mutant & MOLS3 & $0.25 \pm 0.09$ & $5.3 \pm 0.4$ & $1.30 \pm 0.03$ & $300 \pm 20$ & $235 \pm 22$ \\
\hline JW710 & MOLT3 & $0.30 \pm 0.04$ & $11.8 \pm 0.4$ & $0.67 \pm 0.02$ & $130 \pm 14$ & $207 \pm 22$ \\
\hline Rex mutant & MOLT3 & $0.09 \pm 0.00$ & $34.7 \pm 13.5$ & $0.10 \pm 0.01$ & $25 \pm 3$ & $245 \pm 31$ \\
\hline JW710 & MOPS4 & $0.30 \pm 0.07$ & $9.1 \pm 1.8$ & $0.98 \pm 0.02$ & $303 \pm 51$ & $317 \pm 53$ \\
\hline Rex mutant & MOPS4 & $0.27 \pm 0.07$ & $8.4 \pm 1.0$ & $0.98 \pm 0.01$ & $253 \pm 11$ & $266 \pm 11$ \\
\hline JW710 & MOPS3 & $0.35 \pm 0.05$ & $6.1 \pm 0.1$ & $1.20 \pm 0.02$ & $346 \pm 7$ & $303 \pm 7$ \\
\hline Rex mutant & MOPS3 & $0.36 \pm 0.07$ & $7.1 \pm 0.0$ & $1.10 \pm 0.03$ & $300 \pm 16$ & $283 \pm 23$ \\
\hline JW710 & MOPT3 & $0.26 \pm 0.03$ & $7.7 \pm 0.9$ & $0.83 \pm 0.01$ & $196 \pm 11$ & $254 \pm 10$ \\
\hline Rex mutant & MOPT3 & $0.34 \pm 0.02$ & $13.0 \pm 0.5$ & $0.75 \pm 0.01$ & $169 \pm 19$ & $231 \pm 24$ \\
\hline JW710 & MOYPyr & $0.16 \pm 0.01$ & $40.8 \pm 1.6$ & $0.31 \pm 0.01$ & $90 \pm 4$ & $295 \pm 7$ \\
\hline Rex mutant & MOYPyr & $0.19 \pm 0.05$ & $40.5 \pm 5.6$ & $0.31 \pm 0.03$ & $93 \pm 2$ & $307 \pm 19$ \\
\hline $\mathrm{DvH}$ & MOLS4 & N.A. & $6.2 \pm 0.1$ & $0.97 \pm 0.03$ & $182 \pm 12$ & $194 \pm 16$ \\
\hline PhsA mutant & MOLS4 & N.A. & $5.2 \pm 0.4$ & $1.00 \pm 0.01$ & $176 \pm 11$ & $190 \pm 13$ \\
\hline $\mathrm{DvH}$ & MOLS3 & N.A. & $5.7 \pm 0.3$ & $1.21 \pm 0.03$ & $236 \pm 12$ & $223 \pm 18$ \\
\hline PhsA mutant & MOLS3 & N.A. & $6.0 \pm 0.0$ & $1.26 \pm 0.03$ & $214 \pm 7$ & $210 \pm 11$ \\
\hline $\mathrm{DvH}$ & MOLT3 & N.A. & $5.8 \pm 0.2$ & $0.69 \pm 0.09$ & $122 \pm 15$ & $175 \pm 10$ \\
\hline PhsA mutant & MOLT3 & N.A. & $5.4 \pm 0.6$ & $0.64 \pm 0.04$ & $110 \pm 8$ & $172 \pm 11$ \\
\hline $\mathrm{DvH}$ & MOPS4 & N.A. & $6.2 \pm 0.4$ & $1.06 \pm 0.04$ & $240 \pm 13$ & $237 \pm 14$ \\
\hline PhsA mutant & MOPS4 & N.A. & $6.6 \pm 1.3$ & $1.00 \pm 0.06$ & $223 \pm 7$ & $220 \pm 6$ \\
\hline $\mathrm{DvH}$ & MOPS3 & N.A. & $7.8 \pm 0.9$ & $1.19 \pm 0.02$ & $241 \pm 11$ & $228 \pm 14$ \\
\hline PhsA mutant & MOPS3 & N.A. & $5.8 \pm 0.3$ & $1.22 \pm 0.02$ & $226 \pm 19$ & $218 \pm 17$ \\
\hline $\mathrm{DvH}$ & MOPT3 & N.A. & $3.3 \pm 0.2$ & $0.92 \pm 0.01$ & $132 \pm 10$ & $150 \pm 12$ \\
\hline PhsA mutant & MOPT3 & N.A. & $3.3 \pm 0.3$ & $0.86 \pm 0.02$ & $125 \pm 9$ & $142 \pm 6$ \\
\hline $\mathrm{DvH}$ & MOYPyr & N.A. & $22.7 \pm 0.2$ & $0.46 \pm 0.02$ & $65 \pm 16$ & $200 \pm 19$ \\
\hline PhsA mutant & MOYPyr & N.A. & $23.9 \pm 1.8$ & $0.46 \pm 0.04$ & $69 \pm 10$ & $196 \pm 11$ \\
\hline Error dete & $\mathrm{d}$ as & $\mathrm{ard} \mathrm{de}$ & f biolo & replicates & $=3) .{ }^{\mathrm{b}} \mathrm{T}$ & $\begin{array}{l}\text { l protein } \\
76 \text { ), error } \\
\text { lected for } \\
\text {, twice as }\end{array}$ \\
\hline
\end{tabular}


These growth studies allowed several properties of $\operatorname{Rex}_{\mathrm{DvH}}$ to be deduced: 1 ) growth for most cultures was observed immediately after subculture (time points $0-\sim 10 \mathrm{~h}$ ), resulting from carryover in the inoculum $(2 \% \mathrm{vol} / \mathrm{vol}) .2)$ The Rex mutant grew similarly to the parental strain in sulfate-containing medium regardless of the electron donor, although the strains grown with pyruvate compared with lactate were different from each other, i.e. lactate-grown cells had a diauxic growth pattern. 3) The Rex mutant appeared to grow at a slower rate and had a longer lag period in sulfite-containing medium compared to the parental strain, regardless of the electron donor. Additionally, the ratio for either strain between the two media were quite similar ( ratio = LS3/PS3 of the doubling times; parental: 4.2/6.1 0.7; Rex mutant: 5.3/7.1 0.7). This was interpreted to mean that sulfite, and not the electron donor, was affecting the growth of the Rex mutant. 4) The Rex mutant in comparison to the parental strain grew at a slower rate when respiring thiosulfate with pyruvate, and did not grow with lactate as the electron donor. The maximum optical density $\left(\mathrm{OD} 600_{\max }\right)$ for the strains grown with thiosulfate was less than with sulfate or sulfite. 5) The Rex mutant grew similarly as the parental strain when fermenting pyruvate.

Examination of the total protein as determined by Bradford assay (Bradford 1976) at the completion of the experiment (i.e. OD $600_{\max }$ ) revealed two results: 1) respiring cultures grown with pyruvate as the electron donor had a higher total protein per OD600 as compared with lactate, and 2) cultures grown by pyruvate fermentation had similar total protein production as cultures respiring sulfate or sulfite with pyruvate, but not thiosulfate (Table 4-3), confirming previous observations for a similar SRB, D. alaskensis G20 (personal communication with Barbara J. Rapp-Giles). Together, these findings support the importance of Rex in energy conversion pertaining to the available electron acceptor, but likely not the electron donor, and that $\operatorname{Rex}_{\mathrm{DvH}}$ is involved in the metabolism of thiosulfate. 


\section{Section 4. 4. PhsA is not the only thiosulfate reductase in}

\section{DvH}

Because growth of the Rex mutant by respiration (lactate or pyruvate) with thiosulfate was

impaired, additional examination of thiosulfate metabolism was necessary. The genome was examined for genes that code for proteins that may have a role in the reduction of thiosulfate. This query identified several candidates, including: thiosulfate reductase (encoded by DVU0173-2, phs $A B$ ) (Aketagawa et al. 1985), dissimilatory sulfite reductase (encoded by DVU0402-4, dsrABD), (Parey et al. 2010) along with DVU2776, $d s r C$ (Venceslau et al. 2014); and a three gene operon (encoded by DVU3143-45) that may code for a second thiosulfate reductase based on protein sequence comparison to PhsABC from Salmonella. enterica. The role of $p h s A$ was examined for its role in the metabolism of thiosulfate reduction.

To begin to determine the role of PhsAB in thiosulfate reduction, a PhsA transposon mutant was grown on several substrates and compared to its parental strain, wild-type DvH (Figure 4-3) (Table 4-3). Examination of the wild-type strain grown on different substrates revealed several findings. 1) $\mathrm{DvH}$ grown by sulfate respiration with lactate or pyruvate grew with similar rates to each other. Again, the strains grown with lactate, but not pyruvate, had a diauxic growth pattern. These findings were similar to what was observed for JW710 presented above. This was interpreted to mean that carryover from the inoculum provided additional substrates that prevented the immediate transition to the defined medium and is a technical, and not biological, phenomenon. 2) The growth of the wild-type strain by sulfite respiration with lactate appeared typical (i.e. minimal lag prior to entering exponential growth phase, doubling time $\sim 5-8 \mathrm{~h}$, and complete growth within $24 \mathrm{~h}$ ); however, when $\mathrm{DvH}$ was grown by sulfite respiration with pyruvate the growth rate for the wild-type strain 
was slower than with lactate. Furthermore, the wild-type strain did not exhibit an extended lag-phase, as was observed for JW710 discussed previously. This may be likely due to the difference between the two strains. Specifically, JW710 is deleted for uracil phosphoribosyltransferase (upp), a pyrimidine scavenging protein, which may be more necessary when sulfite is provide at relatively high concentrations (40 mM). 3) The growth of the wild-type strain when respiring thiosulfate with lactate was diauxic, as was observed with sulfate, although the growth phenotype between the sulfate- and sulfite-grown cultures are different. Furthermore, the wild-type strain grown by thiosulfate respiration with pyruvate did not experience a lag phase and grew robustly. Finally, the $\mathrm{OD} 600_{\max }$, and thus cell number, for the wild-type samples grown with lactate were less for those strains grown with thiosulfate (OD600 0.7) compared to sulfate (OD600 1.0) or to sulfite (OD600 1.2); although when pyruvate was supplied instead of lactate the max OD600 is less distributed, with all being centered around OD600 1.0.

Next, the PhsA mutant was compared to the wild-type DvH (Figure 4-3) (Table 4-3). The phs A::TnRL-27 mutant grew similarly to that of the wild-type DvH strain for all growth conditions tested, except when grown by thiosulfate respiration with lactate. When the PhsA mutant was grown in this manner it appeared to take an additional $15 \mathrm{~h}$ (i.e. $30 \mathrm{~h}$ time-point versus the $15 \mathrm{~h}$ time-point) before entering into exponential phase, although the growth rates for the two strains during exponential growth were similar. Additionally, the OD $600_{\max }$ between the two strains were similar among the media conditions. Overall, the Phs mutant was impaired in growth compared to the wild-type strain only when grown by thiosulfate respiration with lactate. However, the culture was still able to metabolize thiosulfate as seen by similar rates during exponential growth and OD $600_{\max }$. Therefore, additional enzymes capable of utilizing thiosulfate for energy conversion exist in DvH. As expected, the deletion 
of phs $A B$ and rex together in the JW710 parental strain did prevent growth when respiring thiosulfate with lactate (data not shown), as either deletion of $p h s A$ (in $\mathrm{DvH}$ ) or $\operatorname{rex}$ (in JW710) alone had impaired growth. Therefore, a target of Rex, in addition to PhsA, is essential for thiosulfate metabolism.

\section{Section 4. 5. Rex controls, directly or indirectly, the}

\section{expression of sat, apsA and $\operatorname{dsr} C$}

To determine the role of $\operatorname{Rex}_{\mathrm{DvH}}$ as a transcriptional regulator, the rex marker-less deletion and parental strain were grown by respiration or fermentation as described above and transcripts were assessed by qRT-PCR of early-exponential cultures (Table 4-4). Additional transcript analyses are presented in Table 4-5 and Table 4-6. The targets examined were sat, which had previously been shown to be directly regulated by $\operatorname{Rex}_{\mathrm{DvH}}$ (Christensen et al. 2014), and aps $A, d s r A, d s r C, r e x$, and $\operatorname{phs} A$.

Table 4-4: Transcript analysis of Rex mutant in several different media

\begin{tabular}{|c|c|c|c|c|c|c|}
\hline \multirow[b]{2}{*}{ Media } & \multicolumn{6}{|c|}{ Transcript } \\
\hline & sat & $\operatorname{aps} A$ & $d s r A$ & rex & phs $A$ & $d s r C$ \\
\hline MOLS4 & $4.1 \pm 0.9$ & $0.6 \pm 0.1$ & $0.6 \pm 0.2$ & N.D. & $0.5 \pm 0.2$ & $1.2 \pm 0.2$ \\
\hline MOLS3 & $31.7 \pm 4.9$ & $3.1 \pm 0.3$ & $0.9 \pm 0.1$ & N.D. & $0.2 \pm 0.0$ & $0.7 \pm 0.1$ \\
\hline MOLT3 & $23.6 \pm 5.0$ & $4.6 \pm 0.7$ & $0.5 \pm 0.1$ & N.D. & $1.8 \pm 0.3$ & $0.5 \pm 0.2$ \\
\hline MOPS4 & $10.3 \pm 3.6$ & $1.2 \pm 0.3$ & $0.8 \pm 0.3$ & N.D. & $0.5 \pm 0.2$ & $0.8 \pm 0.2$ \\
\hline MOPS3 & $93.7 \pm 12.0$ & $10.1 \pm 1.6$ & $0.7 \pm 0.2$ & N.D. & $1.2 \pm 0.1$ & $0.7 \pm 0.1$ \\
\hline MOPT3 & $49.7 \pm 8.6$ & $4.1 \pm 0.2$ & $0.4 \pm 0.1$ & N.D. & $1.1 \pm 0.1$ & $0.4 \pm 0.1$ \\
\hline MOYPyr & $1.4 \pm 0.2$ & $0.6 \pm 0.1$ & $0.5 \pm 0.1$ & N.D. & $1.5 \pm 0.2$ & $0.5 \pm 0.1$ \\
\hline
\end{tabular}

Error determined as standard error of the mean among triplicates. Blue and yellow highlight refers to those transcripts that have decreased or increased by greater than twofold relative to the parental strain, JW710, respectively. N.D. not detected. 
Table 4-5: Transcript analysis of Rex mutant and parental strain

\begin{tabular}{cccccc}
\hline & & \multicolumn{4}{c}{${\text { Mean } \mathrm{Cq}^{\mathrm{a}}}$} \\
\cline { 3 - 6 } Name & Media & sat & apsA & dsrA & rex \\
\hline JW710 & MOLS4 & $20.42 \pm 0.13$ & $21.70 \pm 0.10$ & $20.26 \pm 0.05$ & $21.90 \pm 0.12$ \\
Rex mutant & MOLS4 & $18.68 \pm 0.21$ & $22.80 \pm 0.18$ & $20.97 \pm 0.25$ & $>40$ \\
\hline JW710 & MOLS3 & $23.06 \pm 0.09$ & $24.58 \pm 0.09$ & $19.95 \pm 0.03$ & $21.90 \pm 0.08$ \\
$\Delta r e x$ & MOLS3 & $18.33 \pm 0.17$ & $22.58 \pm 0.09$ & $19.90 \pm 0.17$ & $>40$ \\
\hline JW710 & MOLT3 & $22.26 \pm 0.12$ & $23.65 \pm 0.10$ & $19.72 \pm 0.30$ & $21.50 \pm 0.21$ \\
Rex mutant & MOLT3 & $20.30 \pm 0.24$ & $23.84 \pm 0.15$ & $22.73 \pm 0.14$ & $>40$ \\
\hline JW710 & MOPS4 & $21.69 \pm 0.17$ & $22.48 \pm 0.13$ & $20.45 \pm 0.27$ & $21.57 \pm 0.25$ \\
Rex mutant & MOPS4 & $18.55 \pm 0.42$ & $22.09 \pm 0.37$ & $20.63 \pm 0.47$ & $>40$ \\
\hline JW710 & MOPS3 & $23.41 \pm 0.15$ & $24.66 \pm 0.04$ & $18.73 \pm 0.12$ & $20.83 \pm 0.13$ \\
Rex mutant & MOPS3 & $18.49 \pm 0.06$ & $22.29 \pm 0.18$ & $20.21 \pm 0.32$ & $>40$ \\
\hline JW710 & MOPT3 & $23.56 \pm 0.17$ & $24.17 \pm 0.09$ & $20.70 \pm 0.10$ & $22.11 \pm 0.19$ \\
Rex mutant & MOPT3 & $18.93 \pm 0.21$ & $22.56 \pm 0.05$ & $22.39 \pm 0.26$ & $>40$ \\
\hline JW710 & MOYPyr & $20.48 \pm 0.45$ & $21.51 \pm 0.38$ & $22.71 \pm 0.46$ & $24.01 \pm 0.43$ \\
Rex mutant & MOYPyr & $21.24 \pm 0.03$ & $23.91 \pm 0.22$ & $24.70 \pm 0.04$ & $>40$ \\
\hline
\end{tabular}

\begin{tabular}{cccccc} 
& & & & \multicolumn{2}{c}{ reference genes } \\
\cline { 5 - 6 } & & phs & dsrC & $r p l S$ & $r p m C$ \\
\hline JW710 & MOLS4 & $27.19 \pm 0.25$ & $17.08 \pm 0.25$ & $19.97 \pm 0.32$ & $22.62 \pm 0.10$ \\
Rex mutant & MOLS4 & $28.07 \pm 0.30$ & $16.96 \pm 0.18$ & $20.59 \pm 0.28$ & $22.30 \pm 0.39$ \\
\hline JW710 & MOLS3 & $25.88 \pm 0.07$ & $16.38 \pm 0.10$ & $20.10 \pm 0.17$ & $21.85 \pm 0.08$ \\
Rex mutant & MOLS3 & $27.81 \pm 0.11$ & $16.77 \pm 0.22$ & $19.96 \pm 0.05$ & $21.41 \pm 0.27$ \\
\hline JW710 & MOLT3 & $26.73 \pm 0.11$ & $15.60 \pm 0.19$ & $20.14 \pm 0.31$ & $22.51 \pm 0.49$ \\
Rex mutant & MOLT3 & $28.13 \pm 0.13$ & $18.85 \pm 0.38$ & $23.55 \pm 0.32$ & $24.53 \pm 0.11$ \\
\hline JW710 & MOPS4 & $26.93 \pm 0.19$ & $16.25 \pm 0.24$ & $19.53 \pm 0.11$ & $22.61 \pm 0.03$ \\
Rex mutant & MOPS4 & $27.71 \pm 0.39$ & $16.50 \pm 0.32$ & $19.42 \pm 0.33$ & $22.50 \pm 0.29$ \\
\hline JW710 & MOPS3 & $27.18 \pm 0.11$ & $15.41 \pm 0.13$ & $18.77 \pm 0.06$ & $20.41 \pm 0.43$ \\
Rex mutant & MOPS3 & $27.92 \pm 0.05$ & $17.21 \pm 0.11$ & $19.77 \pm 0.12$ & $21.91 \pm 0.32$ \\
\hline JW710 & MOPT3 & $27.57 \pm 0.10$ & $15.25 \pm 0.19$ & $20.70 \pm 0.15$ & $23.14 \pm 0.11$ \\
Rex mutant & MOPT3 & $27.95 \pm 0.08$ & $17.23 \pm 0.24$ & $20.97 \pm 0.07$ & $23.99 \pm 0.10$ \\
\hline JW710 & MOYPyr & $28.55 \pm 0.55$ & $18.71 \pm 0.43$ & $24.17 \pm 0.43$ & $24.65 \pm 0.57$ \\
Rex mutant & MOYPyr & $29.23 \pm 0.16$ & $20.82 \pm 0.01$ & $25.64 \pm 0.34$ & $26.14 \pm 0.15$ \\
\hline
\end{tabular}

${ }^{a}$ Critical threshold $(\mathrm{Cq})$ for specified gene, with technical error determined as standard error of the mean $(n=3)$. 
Table 4-6: Transcript analysis of parental strain, JW710, in several different media Transcript

\begin{tabular}{ccccccc} 
Media & sat & aps $A$ & $d s r A$ & rex & phs $A$ & $d s r C$ \\
\hline MOLS4 & $1.0 \pm 0.1$ & $1.0 \pm 0.1$ & $1.0 \pm 0.4$ & $1.0 \pm 0.1$ & $1.0 \pm 0.2$ & $1.0 \pm 0.2$ \\
MOLS3 & $0.1 \pm 0.0$ & $0.1 \pm 0.0$ & $1.0 \pm 0.1$ & $0.8 \pm 0.1$ & $2.2 \pm 0.2$ & $1.3 \pm 0.1$ \\
MOLT3 & $0.3 \pm 0.1$ & $0.3 \pm 0.1$ & $1.5 \pm 0.5$ & $1.4 \pm 0.3$ & $1.5 \pm 0.3$ & $2.7 \pm 0.6$ \\
MOPS4 & $0.3 \pm 0.0$ & $0.5 \pm 0.0$ & $0.8 \pm 0.2$ & $1.1 \pm 0.2$ & $1.0 \pm 0.2$ & $1.5 \pm 0.2$ \\
MOPS3 & $0.0 \pm 0.0$ & $0.1 \pm 0.0$ & $1.1 \pm 0.2$ & $0.8 \pm 0.1$ & $0.3 \pm 0.0$ & $1.0 \pm 0.1$ \\
MOPT3 & $0.1 \pm 0.0$ & $0.3 \pm 0.0$ & $1.0 \pm 0.1$ & $1.2 \pm 0.2$ & $1.0 \pm 0.1$ & $4.8 \pm 0.6$ \\
MOYPyr & $6.4 \pm 2.6$ & $7.6 \pm 2.5$ & $1.0 \pm 0.4$ & $1.3 \pm 0.5$ & $2.4 \pm 1.1$ & $2.3 \pm 0.8$ \\
\hline
\end{tabular}

Error determined as standard error of the mean. Blue and yellow highlight refers to those transcripts that have decreased or increased by at least twofold, respectively

Examination of the transcript expression of the specified genes of the parental strain revealed three general patterns (Table 4-6), whereby a twofold change was the cutoff requirement for a condition to be considered significantly different by analysis by qRT-PCR. Transcript abundance was normalized for each gene to the parental strain, JW710, grown by sulfate respiration with lactate. These data indicated a few trends: 1) The expression of sat and aps $A$ was decreased when respiring sulfite or thiosulfate with lactate regardless of the electron donor, confirming a previous study (Christensen et al. 2014). Additionally, JW710 cells respiring sulfate with pyruvate also had decreased expression. Furthermore, these genes increased in expression when JW710 was fermenting pyruvate. 2) The expression of $d s r A$, rex, and $p h s A$, remained unchanged across all media conditions tested. Although $p h s A$ transcript did appear to change in expression based on the fold-change differences for several media conditions, further examination of the critical threshold $(\mathrm{Cq})$ revealed that this gene is expressed at very low levels, as evident by a high $\mathrm{Cq}(\mathrm{Cq}>26)$ compared with the other genes assayed (Cq 15-25) in this study (Table 4-5). Therefore, $p h s A$ is most likely 
unchanged across all media conditions tested, or at least is present in low abundance in comparison to other sulfate reduction genes. 3) The expression of $d s r C$ increased in thiosulfate-containing medium, regardless of the electron donor, and while fermenting pyruvate.

The transcript expression results for each of the genes were compared between the Rex mutant and parental strain (Table 4-4). Examination of the expression data of the Rex mutant revealed unique responses for each of the genes assayed. As expected, rex was undetected in the strain deleted for rex. The expression of sat was increased in all respiring cultures compared with the parental strain. Additionally, sat was minimally increased when growing fermentatively but was already increased fourfold from the parental strain while respiring sulfate, consistent with a previous study (Christensen et al. 2014). The expression for aps $A$ increased when respiring sulfite and thiosulfate with lactate or pyruvate when compared with the parental strain grown by sulfate respiration with lactate, but was unchanged with sulfate, regardless of the electron donor, and was also unchanged when grown fermentatively. The expression of $d s r A$ and $p h s A$ were unaffected by the deletion of rex for the conditions examined in this study. Lastly, the expression of $d s r C$ appeared to decrease in the Rex mutant, or at least not increase, for all media, and was only significantly decreased for media that conferred a slowed rate of respiration within the cytoplasm (i.e. thiosulfate-containing or pyruvate fermentation media). Overall, the expression for sat, aps $A$, and $d s r C$, but not $d s r A$ nor $p h s A$, were controlled (directly or indirectly) by Rex $\operatorname{DvHH}_{\text {during }}$ growth on different substrates. Specifically, sat and aps $A$ were shown to be repressed, while $d s \uparrow C$ was activated by Rex $x_{\text {DvH. }}$ For several of these conditions protein abundance, of the encoding genes, had been assayed and determined to correlate reasonably well to the transcript data (Table 4-7). 
Table 4-7: Protein analysis of parental strain and Rex mutant in several different

\begin{tabular}{|c|c|c|c|c|c|c|c|c|}
\hline \multirow[b]{3}{*}{ Strain } & \multirow[b]{3}{*}{ Media } & \multirow[b]{3}{*}{$\begin{array}{l}\text { OD600 } \\
\text { collected }^{a}\end{array}$} & \multicolumn{6}{|c|}{ Protein $^{\mathrm{b}}$} \\
\hline & & & \multicolumn{2}{|c|}{ Sat } & \multicolumn{2}{|c|}{ ApsA } & \multicolumn{2}{|c|}{ DsrA } \\
\hline & & & $\begin{array}{c}\text { Peak } \\
\text { Area } \\
\text { (Avg.) } \\
\end{array}$ & $\begin{array}{c}\text { Peak } \\
\text { Area } \\
\text { (STD) }\end{array}$ & $\begin{array}{c}\text { Peak } \\
\text { Area } \\
\text { (Avg.) }\end{array}$ & $\begin{array}{c}\text { Peak } \\
\text { Area } \\
\text { (STD) } \\
\end{array}$ & $\begin{array}{c}\text { Peak } \\
\text { Area } \\
\text { (Avg.) }\end{array}$ & $\begin{array}{c}\text { Peak } \\
\text { Area } \\
\text { (STD) }\end{array}$ \\
\hline Parental & MOLS4 & $0.26 \pm 0.07$ & 125,602 & 45,644 & 141,372 & 54,081 & 53,401 & 17,584 \\
\hline Rex mutant & MOLS4 & $0.22 \pm 0.04$ & 474,204 & 24,911 & 106,628 & 7,287 & 44,401 & 1,580 \\
\hline Parental & MOLS3 & $0.25 \pm 0.07$ & 19,140 & 1,686 & 12,532 & 240 & 91,475 & 7,926 \\
\hline Rex mutant & MOLS3 & $0.24 \pm 0.04$ & 540,059 & 79,382 & 81,488 & 11,894 & 56,246 & 10,777 \\
\hline Parental & MOLT3 & N.A. & N.A. & N.A. & N.A. & N.A. & N.A. & N.A. \\
\hline Rex mutant & MOLT3 & N.A. & N.A. & N.A. & N.A. & N.A. & N.A. & N.A. \\
\hline Parental & MOPS4 & $0.22 \pm 0.06$ & 32,022 & 16,538 & 46,806 & 29,379 & 29,001 & 24,463 \\
\hline Rex mutant & MOPS4 & $0.25 \pm 0.01$ & 368,808 & 14,558 & 94,105 & 10,649 & 27,573 & 1,867 \\
\hline Parental & MOPS3 & N.A. & N.A. & N.A. & N.A. & N.A. & N.A. & N.A. \\
\hline Rex mutant & MOPS3 & N.A. & N.A. & N.A. & N.A. & N.A. & N.A. & N.A. \\
\hline Parental & MOPT3 & N.A. & N.A. & N.A. & N.A. & N.A. & N.A. & N.A. \\
\hline Rex mutant & MOPT3 & N.A. & N.A. & N.A. & N.A. & N.A. & N.A. & N.A. \\
\hline Parental & MOYPyr & $0.13 \pm 0.00$ & 173,296 & 80,451 & 96,077 & 31,332 & 48,925 & 28,500 \\
\hline \multirow[t]{2}{*}{ Rex mutant } & MOYPyr & $0.12 \pm 0.00$ & 469,933 & 375,822 & 52,569 & 36,988 & 35,319 & 29,186 \\
\hline & & & \multicolumn{2}{|c|}{ Rex } & \multicolumn{2}{|c|}{ DsrC } & \multicolumn{2}{|c|}{ RnfC } \\
\hline \multirow[b]{2}{*}{ Strain } & \multirow[b]{2}{*}{ Media } & \multirow[b]{2}{*}{$\begin{array}{l}\text { OD600 } \\
\text { collected }^{a}\end{array}$} & Peak & Peak & Peak & Peak & Peak & Peak \\
\hline & & & Area & Area & Area & Area & Area & Area \\
\hline Parental & MOLS4 & $0.26 \pm 0.07$ & $\frac{(\text { (Avg.) }}{1,776}$ & $\frac{(\mathrm{STD})}{611}$ & $\begin{array}{l}\text { (Avg.) } \\
78,178\end{array}$ & $\frac{(\mathrm{STD})}{29,978}$ & $\frac{(\text { Avg.) }}{2,465}$ & $\frac{(\mathrm{S1D})}{704}$ \\
\hline Rex mutant & MOLS4 & $0.22 \pm 0.04$ & 46 & 40 & 38,534 & 2,090 & 743 & 22 \\
\hline Parental & MOLS3 & $0.25 \pm 0.07$ & 1,581 & 298 & 97,123 & 651 & 3,213 & 223 \\
\hline Rex mutant & MOLS3 & $0.24 \pm 0.04$ & 53 & 27 & 49,066 & 6,630 & 288 & 30 \\
\hline Parental & MOLT3 & N.A. & N.A. & N.A. & N.A. & N.A. & N.A. & N.A. \\
\hline Rex mutant & MOLT3 & N.A. & N.A. & N.A. & N.A. & N.A. & N.A. & N.A. \\
\hline Parental & MOPS4 & $0.22 \pm 0.06$ & 752 & 525 & 49,387 & 37,637 & 1,757 & 1,329 \\
\hline Rex mutant & MOPS4 & $0.25 \pm 0.01$ & 35 & 22 & 34,630 & 2,291 & 584 & 62 \\
\hline Parental & MOPS3 & N.A. & N.A. & N.A. & N.A. & N.A. & N.A. & N.A. \\
\hline Rex mutant & MOPS3 & N.A. & N.A. & N.A. & N.A. & N.A. & N.A. & N.A. \\
\hline Parental & MOPT3 & N.A. & N.A. & N.A. & N.A. & N.A. & N.A. & N.A. \\
\hline Rex mutant & MOPT3 & N.A. & N.A. & N.A. & N.A. & N.A. & N.A. & N.A. \\
\hline Parental & MOYPyr & $0.13 \pm 0.00$ & 1,226 & 589 & 40,428 & 24,207 & 443 & 243 \\
\hline Rex mutant & MOYPyr & $0.12 \pm 0.00$ & 30 & 14 & 22,747 & 15,441 & 269 & 250 \\
\hline
\end{tabular}

${ }^{a}$ Error determined as standard deviation of biological replicates $(n=3)$. PhsA was not determined. 'brotein determined by selected-reaction monitoring, as described previously by Redding-Johanson et al. (2011). N.A. not assessed. STD standard deviation. 


\section{Section 4. 6. Discussion}

In $\mathrm{DvH}$, many of the genes that code for proteins responsible for dissimilatory sulfate reduction are known, and $\operatorname{Rex}_{\mathrm{DvH}}$ is predicted to regulate several of these genes (Ravcheev et al. 2012). We previously characterized $\operatorname{Rex}_{\mathrm{DvH}}$ for its role as a repressor of sat (Christensen et al. 2014). Here we confirm that Rex $\mathrm{D}_{\mathrm{DvH}}$ controls early steps in the process of sulfate reduction, an observation that was recently reported for Rex in a closely related sulfate reducer, D. alaskensis G20 (Kuehl et al. 2014). Specifically, Rex altered the expression of genes that code for proteins responsible for the activation and reduction of sulfate to sulfite (i.e. sat and aps $A$ ). Furthermore, the strain deleted for rex was impaired for growth in thiosulfate-containing medium.

To gain insight into what genes were necessary for sulfate reduction and how Rex may be involved, a whole-genome gene fitness study was performed on a parental DvH strain grown by sulfate respiration with lactate and compared to a strain deleted for rex (Table 4-1). Interestingly, the fitness value for only a small number of genes was different between the two strains and the differences observed were minor (typically less than twofold), which is in contrast to previous fitness studies that examined DvH under different stresses (e.g. minimal vs rich media (Fels et al. 2013) or nitrate stress (Korte et al. 2014)). This was interpreted to mean that the deletion of rex was not detrimental to growth by sulfate respiration with lactate. This result was expected as the deletion of rex had been shown to increase the expression of sat (Christensen et al. 2014) and presumably the capacity to reduce sulfate.

Further examination of the gene fitness results revealed that the quinone reductase operon ( $\operatorname{qr} A B C D)$ had decreased fitness (i.e. became more negative) in the Rex mutant compared with the parental strain; although, these genes were considered to be non-essential 
regardless of the strain (Table 4-1). QrcABCD is important for the proper metabolism of sulfate reduction with lactate in SRB as the deletion of $\operatorname{qr} A B C D$ appeared to be delayed for growth and have slightly lower growth rates compared to the parental strain (data not shown). Specifically, QrcABCD transfers electrons from the periplasm to the cytoplasm for sulfate reduction with the quinone-interacting membrane-bound oxidoreductase (QmoABC) as an intermediate (Keller et al. 2014) (Figure 4-4). However, electrons also feed into QmoABC via lactate oxidation through lactate dehydrogenase, thereby bypassing QrcABCD and allowing for sulfate reduction to proceed (Keller et al. 2014). For SRB, the deletion of rex increased expression of $q r A B C D$ along with many of the genes that code for proteins responsible for the activation of sulfate to APS and the reduction of APS to sulfite, including sat, qmo $A B C D$, and apsBA. (Christensen et al. 2014; Kuehl et al. 2014). Therefore, when either of the genes in the $\operatorname{qr} A B C D$ operon are also deleted in the Rex mutant strain the electrons are unable to be transferred from the periplasm to APS reductase. However, electrons can still feed into sulfate reduction via lactate oxidation. The reason for the qrCABCD deletion being more detrimental to the Rex mutant as compared to the parental strain is most likely due to the upregulation of sat, which would squander the ATP. Consequently, by increasing the sulfate activation enzyme, Sat, which is reversible, but not the enzyme responsible for transferring electrons generated within the periplasm to APS reduction, the cell is unable to reduce APS and instead APS converts back to sulfate and consumes an ATP molecule. 

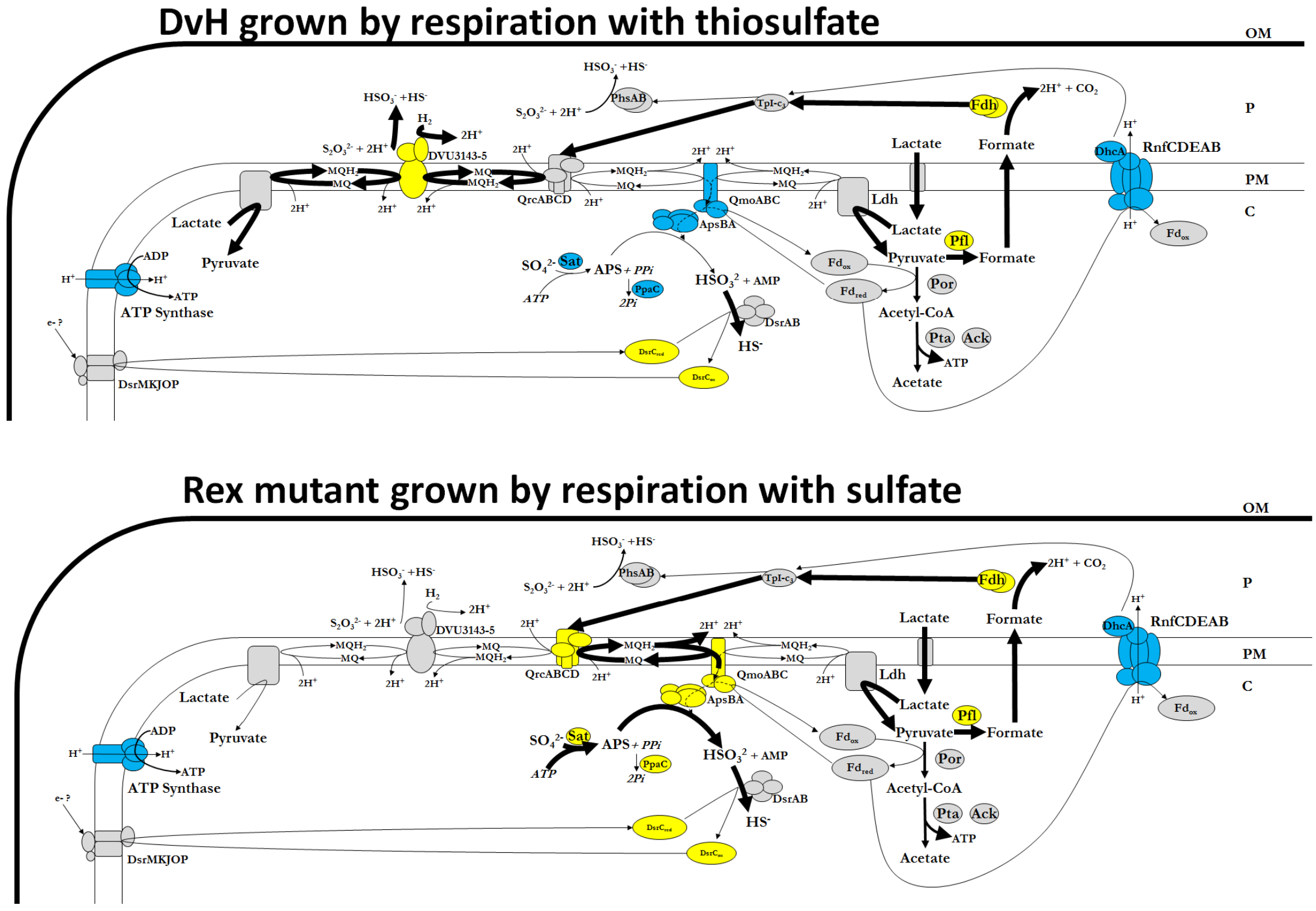
Figure 4-4: Sulfate reduction in DvH. Gene expression results for (TOP) DvH grown by thiosulfate respiration with lactate as compared to sulfate respiration with lactate presented in a new way from www.microbesonline.org (Dehal et al. 2010) and (BOTTOM) Rex mutant grown by respiration with sulfate as compared to parental strain (Kuehl et al. 2014). Thickness of arrows denote the presumed flux as determined by gene expression. Changes in gene expression by at least twofold between DvH grown by thiosulfate respiration compared to sulfate respiration with lactate are color coded: yellow (increase), blue (decrease), grey (unchanged). TpI-c 3 , cytochrome c 3 ; QrcABCD, Quinone reductase complex; MQ, menaquinone; QmoABC, quinone-interacting membrane-bound oxidoreductase; ApsBA, adenylylsulfate reductase; DsrMKJOP, membrane portion of dissimilatory sulfite reductase; DsrAB and DsrC, dissimilatory sulfite reductase; Sat, sulfate adenylyltransferase; Ldh, lactate dehydrogenase; Por, pyruvate oxidoreductase; Pta, phosphate acetyltransferase; Ack, acetate kinase; Fd, ferredoxin; PhsAB, thiosulfate reductase; DhcA, decaheme cytochrome c; RrfCDEAB, NADH:quinone oxidoreductase; $\mathrm{SO}_{4}{ }^{2-}$, sulfate; APS, adenosine phosphosulfate; $\mathrm{SO}_{3}{ }^{2-}$, sulfite; $\mathrm{S}^{2-}$, sulfide; $\mathrm{S}_{2} \mathrm{O}_{3}{ }^{2-}$, thiosulfate; $\mathrm{OM}$, outer membrane; $\mathrm{P}$, periplasmic space; $\mathrm{PM}$, periplasmic membrane; C, cytoplasm. Thiosulfate reductase is predicted to function within the periplasm in $\mathrm{DvH}$ as it does for Archaeoglobus fulgidus (Hocking et al. 2014) and S. enterica (Stoffels et al. 2012), although no transmembrane component is currently known to be associated with PhsAB in $\mathrm{DvH}$. 
In contrast to the $\operatorname{qr} A B C D$ operon, the deletion of any gene within the ATP synthase operon, atpF1F2HAGDC, increased the fitness (i.e. became more positive) in the Rex mutant compared to the parental strain, except atp $C$, although several fitness values were close to the cutoff of being considered essential (i.e. $\left.\log _{2}=-1.7-2.2\right)$ (Table 4-2). The atp $E B$ operon, which encode $F_{o}$ subunits $A$ and $C$ however, were still essential. Furthermore, transcript analyses of atpF1F2HAGDC, as performed by microarray, for $\mathrm{DvH}$ grown by sulfite or thiosulfate respiration with lactate were decreased in expression compared to when respiring sulfate with lactate (Dehal et al. 2010) (Table 4-8). The decreased gene expression while respiring most substrates was interpreted to mean that this operon is only essential at high concentrations when respiring sulfate with lactate. The deletion of rex in SRB (Table 4-4) has shown that genes that code for proteins necessary for the reduction of sulfate to sulfite and the synthesis of ATP (e.g. sat, $p p a C$, adk, atpF1F2HAGDC) are increased in expression. We interpret these findings to mean that genes that code for proteins responsible for ATP synthesis are overexpressed in the Rex mutant and that their increased abundance may disrupt sulfate reduction. 
Table 4-8: DvH Transcript analysis as determined by Microarray

\begin{tabular}{|c|c|c|c|c|c|c|c|}
\hline \multirow[b]{3}{*}{ DVU } & \multirow[b]{3}{*}{ name } & & \multicolumn{5}{|c|}{ Experiment } \\
\hline & & & 389 & 1301 & 388 & 1737 & 7241 \\
\hline & & transcript ${ }^{*}$ & LS3 & LT3 & PS4 & PT3 & Pyr \\
\hline 0172 & $p h s B$ & -13.63 & -0.7 & -1.7 & 0.1 & -0.5 & -0.6 \\
\hline 0173 & $p h s A$ & -13.90 & -0.3 & -0.6 & 0.0 & -0.4 & N.A \\
\hline 0384 & $f l r$ & -13.15 & -0.4 & -0.7 & -0.6 & -0.4 & 0.7 \\
\hline 0402 & $d s r A$ & -9.33 & -0.8 & -1.3 & 0.2 & 0.2 & 0.9 \\
\hline 0403 & $d s r B$ & -9.46 & -0.9 & -1.2 & -0.1 & -0.7 & 0.9 \\
\hline 0404 & $d s r D$ & -8.76 & 0.3 & 0.0 & 0.7 & 0.7 & 0.1 \\
\hline 0414 & tme & -13.70 & -0.8 & -2.0 & 0.4 & -0.9 & 0.4 \\
\hline 0429 & $e c h F$ & -14.95 & -0.5 & -0.3 & 0.4 & 0.9 & 1.7 \\
\hline 0430 & $e c h E$ & -9.46 & N.A. & N.A. & N.A. & N.A. & N.A. \\
\hline 0431 & $e c h D$ & -14.79 & -0.5 & -1.7 & 0.5 & 0.6 & 2.0 \\
\hline 0432 & $\operatorname{ech} C$ & -14.48 & 0.1 & -1.8 & 0.7 & 0.3 & 2.0 \\
\hline 0433 & $\operatorname{ech} B$ & -14.98 & -0.5 & -1.1 & 0.4 & 0.4 & 2.4 \\
\hline 0434 & $\operatorname{ech} A$ & -13.99 & -0.6 & -0.5 & 0.2 & -0.3 & \\
\hline 0531 & $b m c F$ & -15.55 & 0.1 & 0.8 & 0.6 & 1.2 & 3.6 \\
\hline 0532 & hmcE & -15.19 & -0.2 & -0.2 & 0.3 & 0.5 & 3.1 \\
\hline 0533 & $b m c D$ & -15.62 & -0.5 & -0.6 & 0.2 & 0.3 & 2.4 \\
\hline 0534 & bmcC & -14.72 & -0.3 & -1.5 & 0.4 & -0.4 & N.A. \\
\hline 0535 & $b m c B$ & -14.86 & -0.2 & 1.1 & 0.4 & 1.4 & N.A. \\
\hline 0536 & $b m c A$ & -15.09 & 0.1 & 0.7 & 0.4 & 1.9 & N.A. \\
\hline 0587 & $f d h A$ & -13.36 & N.A. & N.A. & N.A. & N.A. & N.A. \\
\hline 0588 & $f d h B$ & -13.92 & 1.1 & 0.5 & 1.6 & 1.8 & -0.7 \\
\hline 0600 & $l d h$ & -14.97 & 0.0 & -0.3 & 0.7 & -0.3 & -0.3 \\
\hline 0687 & aor-2 & -14.21 & -0.5 & -0.4 & -0.1 & -1.4 & N.A. \\
\hline 0692 & $\operatorname{qrcD}$ & -12.54 & -0.7 & -0.9 & -0.7 & -0.9 & 1.1 \\
\hline 0693 & $\operatorname{qrc} C$ & -12.55 & -0.8 & -1.5 & -0.9 & -0.3 & 2.1 \\
\hline 0694 & $\operatorname{qrcB}$ & -12.59 & -1.2 & -2.2 & -1.2 & -0.1 & 1.0 \\
\hline DORF6830 & $\operatorname{qrc} A$ & N.A. & N.A. & N.A. & N.A. & N.A. & N.A. \\
\hline 0774 & $\operatorname{atp} C$ & -11.90 & -0.9 & -0.5 & -0.8 & -1.2 & 0.2 \\
\hline 0775 & $\operatorname{atpD}$ & -12.14 & -1.5 & -1.9 & -1.2 & -1.1 & 0.3 \\
\hline 0776 & $\operatorname{atp} G$ & -11.58 & -1.3 & -2.3 & -0.8 & -1.2 & 0.9 \\
\hline 0777 & $\operatorname{atp} A$ & -11.33 & -1.7 & -3.1 & -1.5 & -1.0 & 0.8 \\
\hline 0778 & atpH & -12.57 & -1.2 & -2.0 & -0.9 & -0.8 & 0.9 \\
\hline 0779 & atpF1 & -12.11 & -1.4 & -2.2 & -1.0 & -0.8 & 0.4 \\
\hline 0780 & atpF1 & -10.61 & -0.1 & -0.5 & -0.7 & -0.8 & 0.3 \\
\hline 0819 & $i s f-1$ & -13.09 & -1.8 & -1.2 & -0.5 & -0.3 & 1.3 \\
\hline 0846 & $a p s B$ & -8.75 & -1.4 & -1.6 & -0.4 & -1.4 & 1.5 \\
\hline 0847 & $\operatorname{aps} A$ & -8.93 & -2.0 & -2.4 & -1.0 & -1.6 & 1.2 \\
\hline 0848 & $q m o A$ & -11.01 & -1.4 & -2.3 & -1.3 & -3.3 & 0.5 \\
\hline 0849 & $q m o B$ & -12.03 & -1.6 & -1.8 & -0.8 & -2.1 & 1.0 \\
\hline 0850 & $q m o C$ & -12.82 & -1.8 & -2.7 & -0.9 & -1.0 & 1.3 \\
\hline 0851 & $q m o D$ & -12.10 & -0.6 & -0.6 & -0.6 & -1.4 & 1.1 \\
\hline
\end{tabular}




\begin{tabular}{|c|c|c|c|c|c|c|c|}
\hline DVU & name & transcript ${ }^{*}$ & LS3 & LT3 & PS4 & PT3 & Pyr \\
\hline 1165 & $n d h$ & -14.97 & -0.3 & -0.3 & 0.2 & -0.2 & -0.1 \\
\hline 1179 & aor & -10.61 & -0.6 & -0.6 & -0.2 & -0.7 & N.A. \\
\hline 1286 & $d s r P$ & -11.59 & N.A. & N.A. & N.A. & N.A. & 0.9 \\
\hline 1287 & $d s r O$ & -12.22 & -0.8 & -1.5 & -0.3 & 0.3 & 1.1 \\
\hline 1288 & $d s r J$ & -12.23 & N.A. & N.A. & N.A. & N.A. & 1.0 \\
\hline 1289 & $d s r K$ & -12.61 & -1.1 & -1.9 & -0.7 & -0.2 & 1.3 \\
\hline 1290 & $d s r M$ & -12.28 & N.A. & N.A. & N.A. & N.A. & N.A. \\
\hline 1295 & sat & -9.21 & -1.4 & -2.0 & -2.2 & -2.5 & 0.4 \\
\hline 1636 & $p p a C$ & -11.13 & -0.6 & -1.2 & -1.0 & -1.1 & 1.1 \\
\hline 1769 & byd $A$ & -13.71 & -0.7 & -0.5 & -0.4 & 0.1 & 0.6 \\
\hline 1770 & bydB & -13.94 & -0.3 & -0.6 & -0.3 & 0.1 & N.A. \\
\hline 1917 & bys $B$ & -11.77 & -1.0 & -2.0 & -0.4 & -0.5 & -0.1 \\
\hline 1918 & bys $A$ & -11.22 & N.A. & N.A. & N.A. & N.A. & N.A. \\
\hline 1921 & bynB-1 & -12.91 & -1.2 & -2.0 & -0.5 & -2.2 & 1.1 \\
\hline 1922 & byn $A-1$ & -13.26 & -1.5 & -2.3 & -0.6 & -2.4 & 1.0 \\
\hline 1932 & $a d k$ & -9.25 & -1.0 & -0.3 & -1.2 & -1.6 & 0.4 \\
\hline 1974 & ndh-likee & -13.43 & 0.7 & 1.0 & 1.2 & -0.2 & N.A. \\
\hline 2110 & $b 2975$ & -14.50 & 0.8 & 0.0 & -1.3 & -2.3 & N.A. \\
\hline 2271 & pflA & -15.41 & -0.2 & 0.2 & -0.4 & 0.9 & 0.9 \\
\hline 2272 & $p f l D$ & -15.33 & -1.0 & -0.6 & -1.2 & 0.6 & N.A. \\
\hline 2285 & & -13.52 & 0.1 & -0.9 & -1.0 & -0.1 & -0.2 \\
\hline 2286 & $\operatorname{coo} M$ & -12.35 & -0.4 & -1.4 & -1.5 & -1.6 & -0.2 \\
\hline 2287 & $\operatorname{cooK}$ & -12.13 & N.A. & N.A. & N.A. & N.A. & -0.3 \\
\hline 2288 & cooL & -11.96 & -0.2 & -1.8 & -1.6 & -1.5 & -0.4 \\
\hline 2289 & $\cos X$ & -11.92 & -0.6 & -1.6 & -1.5 & -0.9 & -0.4 \\
\hline 2290 & $\operatorname{coo} U$ & -11.55 & -0.3 & -1.6 & -1.5 & -1.4 & -0.5 \\
\hline 2291 & $\mathrm{cooH}$ & -11.83 & -0.1 & -0.9 & -1.5 & -1.3 & -0.9 \\
\hline 2292 & $\operatorname{coo} A$ & -10.83 & -0.3 & -1.2 & -1.0 & -1.6 & -0.3 \\
\hline 2293 & $\mathrm{cooF}$ & -10.95 & -0.3 & -0.7 & -0.8 & -1.1 & -0.7 \\
\hline 2397 & & -12.50 & 0.1 & -1.7 & 0.5 & 0.0 & 0.4 \\
\hline 2398 & & -12.24 & -1.0 & -2.1 & 0.2 & -0.6 & 0.5 \\
\hline 2399 & & -11.82 & -1.2 & -1.9 & -0.5 & -1.0 & 0.3 \\
\hline 2400 & & -11.50 & -0.9 & -1.7 & -0.5 & -1.1 & 1.1 \\
\hline 2401 & & -12.08 & -0.6 & -1.7 & 0.0 & -0.6 & 0.2 \\
\hline 2402 & $b d r A$ & -12.04 & -1.2 & 1.7 & -0.1 & -1.3 & 0.6 \\
\hline 2403 & $b d r B$ & -11.51 & -1.1 & -1.8 & -0.2 & -1.2 & -0.4 \\
\hline 2404 & $b d r C$ & -11.17 & -0.9 & -1.7 & 0.2 & -1.2 & -0.6 \\
\hline 2405 & $a d h$ & -9.08 & -0.4 & -1.3 & 0.8 & 0.6 & N.A. \\
\hline 2451 & & -12.96 & 0.0 & -0.5 & -4.0 & -2.4 & -0.7 \\
\hline 2481 & $c f d B$ & -13.00 & -0.3 & -1.2 & 0.2 & -0.9 & 0.0 \\
\hline 2482 & $c f d A$ & -13.65 & N.A. & N.A. & N.A. & N.A. & N.A. \\
\hline DVU & name & transcript ${ }^{*}$ & LS3 & LT3 & PS4 & PT3 & Pyr \\
\hline
\end{tabular}




\begin{tabular}{|c|c|c|c|c|c|c|c|}
\hline 2525 & bynB-2 & -14.61 & -1.2 & -0.8 & -0.5 & -0.5 & 1.4 \\
\hline 2526 & byn $A-2$ & -14.09 & -1.0 & -0.1 & -0.4 & -0.8 & 0.6 \\
\hline 2673 & $g / p D$ & -13.32 & -0.1 & -0.5 & -0.2 & 0.3 & 0.5 \\
\hline 2674 & $s d b B$ & -13.92 & -0.2 & -1.4 & 0.0 & -0.1 & 0.1 \\
\hline 2683 & & -13.18 & -0.9 & -0.3 & -1.4 & -0.1 & -0.7 \\
\hline 2776 & $d s r C$ & -10.26 & 0.1 & -0.4 & -0.2 & -0.3 & N.A. \\
\hline 2783 & & -13.49 & 0.8 & 0.4 & -0.5 & -1.4 & -0.6 \\
\hline 2784 & IldD & -13.58 & 0.5 & -0.2 & -0.6 & -1.2 & -0.9 \\
\hline 2791 & $d h c A$ & -12.62 & 0.2 & -0.5 & -0.3 & -0.9 & 0.7 \\
\hline 2792 & $r n f C$ & -13.44 & -0.8 & -1.8 & -0.2 & -0.9 & 1.0 \\
\hline 2793 & $r m f D$ & -14.08 & -0.3 & -1.7 & 0.0 & -0.6 & 0.9 \\
\hline 2794 & $\operatorname{rnf} G$ & -13.70 & -0.5 & -1.2 & -0.5 & -0.7 & 0.8 \\
\hline 2795 & $r n f R$ & -14.68 & -0.6 & -1.7 & -0.5 & -0.8 & 0.4 \\
\hline 2769 & $\operatorname{rnf} A$ & -14.40 & -0.5 & -1.4 & -0.3 & -0.2 & 0.6 \\
\hline 2797 & $\operatorname{rnfB}$ & -13.60 & -0.3 & -0.9 & 0.0 & -0.5 & 1.2 \\
\hline 2798 & $a p b E$ & -13.29 & 0.0 & -0.2 & 0.3 & -0.3 & 0.6 \\
\hline 2809 & $f d h c_{3}$ & -15.16 & 0.0 & 0.2 & 0.2 & 1.2 & -1.5 \\
\hline 2810 & $f d h E$ & -15.35 & -0.2 & 0.3 & 0.0 & 0.7 & -1.2 \\
\hline 2811 & $f d h B$ & -15.60 & 0.2 & 0.7 & 0.2 & 0.7 & -2.0 \\
\hline 2812 & $f d h A$ & -15.40 & N.A. & N.A. & N.A. & N.A. & -2.7 \\
\hline 3025 & por & -10.82 & -0.8 & -2.1 & -1.4 & -1.3 & 0.5 \\
\hline 3026 & lldP & -12.91 & -1.4 & -1.9 & -2.9 & -2.3 & 0.2 \\
\hline 3027 & $g l c D$ & -11.66 & -1.3 & -2.4 & -1.4 & -1.4 & 0.8 \\
\hline 3028 & $g l p C$ & -12.21 & -1.2 & -1.4 & -1.4 & -1.6 & -0.3 \\
\hline 3029 & pta & -11.85 & -0.9 & -2.9 & -1.3 & -1.7 & -0.1 \\
\hline 3030 & ack $A$ & -11.86 & -1.1 & -2.7 & -1.8 & -1.3 & 0.3 \\
\hline 3031 & pta-like & -11.76 & -0.9 & -2.6 & -1.9 & -2.2 & 0.0 \\
\hline 3032 & & -12.13 & -1.6 & -2.5 & -1.4 & -1.2 & 0.4 \\
\hline 3033 & & -12.18 & -1.4 & -2.1 & -1.1 & -0.7 & N.A. \\
\hline 3035 & & -13.35 & -0.4 & -0.7 & -0.3 & -1.1 & 0.0 \\
\hline 3143 & $\not h s B$ & -15.98 & -1.2 & -0.4 & -0.7 & 2.9 & 2.2 \\
\hline 3144 & phs $A$ & -15.38 & -0.7 & -1.4 & 0.4 & 3.4 & 1.2 \\
\hline 3145 & $\mathrm{PhsC}$ & -15.40 & 0.1 & 0.1 & 0.1 & 2.7 & 1.2 \\
\hline 3171 & TpI-c3 & & -0.3 & -1.3 & 0.10 & -1.4 & 1.3 \\
\hline 3212 & nox & -12.97 & -1.6 & -1.3 & 0.1 & -0.5 & 0.2 \\
\hline 3261 & frdC & -14.00 & 0.3 & 0.1 & -0.5 & -0.6 & -0.6 \\
\hline 3262 & $\operatorname{frd} A$ & -14.60 & -0.2 & -1.0 & -0.4 & -0.6 & -0.5 \\
\hline 3263 & $f r d B$ & -14.26 & 0.3 & -0.5 & -0.3 & -0.6 & 0.3 \\
\hline 3284 & $b 2975$ & -15.33 & 0.3 & 1.9 & -0.4 & 1.1 & -0.3 \\
\hline
\end{tabular}

\#Microarray experiments previously performed on DvH cultures www.microbesonline.org (Dehal et al. 2010). Each sample was normalized to the culture grown in defined lactate-sulfate medium (LS4D) and presented in $\log _{2}$ format. Experiment number: 389, 60 mM Lactate with $40 \mathrm{mM}$ sulfite; 1301, $60 \mathrm{mM}$ lactate with $30 \mathrm{mM}$ thiosulfate; $388,120 \mathrm{mM}$ pyruvate with 30 $\mathrm{mM}$ sulfate; 1737, $120 \mathrm{mM}$ pyruvate with $30 \mathrm{mM}$ thiosulfate; and $7241,120 \mathrm{mM}$ pyruvate (fermentation). Blue and yellow highlight refers to those transcripts that have decreased or 
increased by greater than twofold, respectively.* Microarray study (Wall et al. 2008) for DvH grown by sulfate respiration with lactate in LS4D medium, $\log _{2} \mathrm{R}$ format, average $=13.7$. mRNA transcript is normalized to gDNA. N.A. Not applicable.

Because the Rex mutant strain is able to grow when any gene in the atpF1F2HAGDC operon was also deleted, and these genes were considered to be nonessential based on fitness scores (at least in the Rex mutant), other mechanisms for ATP generation must be compensating for the loss of ATP synthase in these strains. For D. alaskensis G20, a strain deleted for rex has been shown to have increased expression in the genes needed for formate production and metabolism (e.g. $p f l$ and $f d h A B E-$ - 3 ), as well as hydrogen production at the periplasm linked to proton translocation (e.g. echABCDEF), and a high molecular weight membrane-bound electron carrier predicted to transfer electrons across the membrane (e.g. hmc $A B C D E F$ ) (Kuehl et al. 2014). Interestingly, genes that code for proteins responsible for substrate level phosphorylation via pyruvate oxidation to acetate (e.g. por, pta, and ack) remained unchanged. Genes that code for proteins responsible for other processes were decreased in expression, including periplasmic hydrogenase (e.g. bys); and the membrane-bound Rnf complex $(d h c A-m f C D E A B)$ that is associated with proton translocation and electron flow as well as $\mathrm{NAD}^{+}$reduction. These findings were interpreted to mean that formate production (via pyruvate formate lyase, $\mathrm{Pfl}$ ) and not acetyl-CoA production (via pyruvate oxidoreductase, Por) from pyruvate allowed the electrons to move directly to the periplasm (as formate) to be transferred to TpI-c 3 and subsequently to QrcABCD for the reduction of sulfate. Two protons are also released when formate is oxidized which contribute to the proton gradient and subsequently to ATP synthesis. Therefore, in the strain deleted for rex, other mechanisms for ATP generation increased which may compensate and increase gene fitness when atpF1F2HAGDC is deleted in the Rex mutant compared with the parental strain. 
Rex proteins have been shown to respond to changes in the redox state of the cell through the $\mathrm{NADH} / \mathrm{NAD}^{+}$ratio, and adjust gene expression in order to maintain this ratio (Gyan et al. 2006). Typically, Rex proteins repress genes that code for proteins that oxidize NADH. For example, when cells are grown by respiration, Rex represses such genes as lactate dehydrogenase (ldh) and acetaldehyde dehydrogenase (adhE2) as observed in Clostridium acetobuylicum (Wietzke et al. 2012) and Staphylococcus aureus (Pagels et al. 2010), and NADH dehydrogenase (nuo or $n d h$ ) as shown in Thermotoga maritima (Ravcheev et al. 2012), Streptomyces coelicolor A3(2) (Brekasis et al. 2003), and Bacillus subtilis (Gyan et al. 2006). NADH is presumed to increase when respiration is slowed or halted (Williamson et al. 1967). As expected, genes that code for proteins responsible for NADH oxidation were increased under fermentation conditions, as observed for adbE, $a d b I$ and $l d h$ in Staphylococcus aureus (Pagels et al. 2010). Therefore, it was concluded that Rex represses genes that code for proteins that oxidize $\mathrm{NADH}$, and this repression is removed upon increasing $\mathrm{NADH}$ concentration.

In SRB, slowed respiration rates would occur when a terminal electron acceptor (i.e. sulfate, sulfite or thiosulfate) is not readily available, and the relative concentration of NADH is presumed to increase when fermenting pyruvate (Meyer et al. 2014). Therefore, we hypothesized that when a cell lacked a terminal electron acceptor, the cell would increase expression for genes that code for proteins responsible for oxidizing NADH as discussed above. Examination of the transcript expression results for DvH grown by pyruvate fermentation revealed genes that code for proteins responsible for sulfate reduction were increased, including: qrc $A B C D$, qmo $A B C D$, aps $B A$, sat, $p p a C$, adk, but not enzymes responsible for the oxidation of NADH (e.g. nox and $n d h$,), although $d h c A-\operatorname{mfCDGR} A B \operatorname{did}$ appear to increase slightly (Table 4-8) (Dehal et al. 2010) (Table 4-6). Interestingly, the genes 
predicted to be regulated by Rex in SRB typically code for proteins in sulfate reduction (Ravcheev et al. 2012). One of these, DhcA-RnfCDEAB, is known to play a role in NADH oxidation. The encoding operon has also been experimentally confirmed to be activated and not repressed by Rex in D. alaskensis G20 by microarray (Kuehl et al. 2014). Therefore, in SRB, it appeared that Rex increased respiration processes, potentially to scavenge nutrients from the environment, as opposed to oxidizing NADH to maintain steady-state $\mathrm{NAD}^{+}$ concentrations. To elaborate on this hypothesis, the overexpression of sat in $\mathrm{DvH}$ had shown increased sulfate uptake for a culture starved for a terminal electron acceptor, and conversely the deletion of sat decreased sulfate uptake (personal communication with Grant Zane). The deletion of rex also had increased sulfate uptake. As sat is de-repressed during fermentation by Rex, as well as increased in expression when rex is deleted, it is reasonable to presume that Rex may control sulfate uptake through the expression of sat. The exact mechanism for how Sat increases sulfate uptake is not currently known.

To understand better the role that Rex may have on controlling sulfate reduction, a strain deleted for rex was grown on different substrates and compared to a parental strain (Table 4-2). As Rex has been shown to respond to the $\mathrm{NADH} / \mathrm{NAD}^{+}$ratio, the deletion of rex should prevent the cell from responding to changes in this ratio. In fact, the deletion of Rex should resemble the state at which Rex is de-repressed, which would be similar to when starved for an electron donor, i.e. pyruvate fermentation. Under these circumstances, proteins involved in sulfate reduction are presumed to be increased as the expression of the genes that code for these proteins is increased. The deletion of rex did not affect growth by respiration with sulfate nor by pyruvate fermentation compared to the parental strain. However, the deletion of rex did appear to delay growth when sulfite was provided as the electron donor. Nevertheless, the rates between the Rex mutant and parental strain were 
similar. The parental strain, JW710, is deleted for uracil phosphoribosyltransferase (upp), a pyrimidine salvaging enzyme. To our knowledge, the deletion of this gene is not known to be a stress for the cell. Examination of the wild-type strain, $\mathrm{DvH}$, in this study did reveal that the rates were similar to JW710. However, the wild-type strain did not experience an initial lag that had been observed for JW710. Furthermore, high concentrations of sulfite is a known stress for DvH (Badziong et al. 1978; Pereira et al. 2008), and the concentration used in this study (40 mM) may have exceeded the toxic levels, and only once sulfite levels had decreased (i.e. detoxified), or cell numbers were increased, was sulfite efficiently coupled to energy conversion for growth. Therefore, the deletion of $u p p$, in addition to the sulfite toxicity, may be the reason for the longer lag prior to exponential growth for the parental strain and even more so with the Rex mutant than the wild-type DvH strain. Finally, the deletion of rex decreased the rate of growth when respiring thiosulfate, regardless of the electron donor. Therefore, from growth experiments, the deletion of rex appeared to disrupt the metabolism of thiosulfate, but not any other electron acceptor. Additionally, the provided electron donor, lactate or pyruvate, did not appear to affect the Rex mutant compared to the parental strain.

If the role of Rex was specific to respiration versus fermentation, as previously alluded to, then there should not have been as drastic (or any) difference in growth between the parental and Rex mutant strain when grown by respiration with sulfate-, sulfite-, or thiosulfate-containing media, unless there was something different between the reduction of sulfate and sulfite and the metabolism of thiosulfate. Interestingly, sulfate and sulfite are reduced within the cytoplasm, whereas thiosulfate is presumed to be reduced in the periplasm (Stoffels et al. 2012). Bacteria have been shown to metabolize thiosulfate by at least two distinct processes. Firstly, thiosulfate can be reduced to sulfite and sulfide by a two- 
electron reduction reaction, as was shown in vitro for a $16 \mathrm{kDa}$ enzyme in $\mathrm{DvH}$ (Findley et al. 1970; Haschke et al. 1971). Or secondly, it could be disproportionated to sulfate and sulfide in the presence of water (Ravot et al. 1995); however, no enzyme capable of the latter process has been identified in DvH (Finster et al. 1998; Heidelberg et al. 2004). The deletion of qmo $A B C$ is impaired for growth only on sulfate, and not sulfite or thiosulfate, which was interpreted to mean that thiosulfate is converted to sulfite and not sulfate in DvH (Zane et al. 2010). The hypothesis was presented that Rex $x_{\text {DvH }}$ may be necessary for altering the electron flow between cytoplasmically and periplamically located reduction processes and not specifically to increasing sulfate reduction rates.

To begin to address how the electrons are transferred to a thiosulfate reductase located within the peirplasm (or periplsmic membrane) in $\mathrm{DvH}$, and how Rex may disrupt this transfer, we first looked for genes that increased in expression in the presence of thiosulfate as compared to sulfate (Dehal et al. 2010) (Table 4-8). In these studies, genes that were increased in expression included: two of the three formate dehydrogenases $(f d h A B$ and $\left.f d h A B E_{3}\right)$, pyruvate formate lyase ( $\left.p f{ }^{\prime}\right)$, a high-molecular weight cytochrome c complex that is located within the periplasmic membrane $(h m c A B C D E F)$, a putative lactate permease (DVU3284) and DVU3143-5. In the transcript studies presented here (Table 4-6), $d s r C$ was also shown to increase in thiosulfate-containing medium. Interestingly, genes that code for proteins responsible for early steps in sulfate reduction (e.g. sat, aps $B A$, qmo $A B C D$, and $p p a C$ ) and substrate-level phosphorylation (e.g. por, pta and ack) decreased in expression. These findings were interpreted to mean that the cell was switching away from sulfate reduction (although genes that code for proteins responsible for sulfite reduction [e.g. $d s r A B D$ ] remained unchanged) and instead to thiosulfate reduction by a currently unknown mechanism when thiosulfate is the sole electron acceptor. 
Next, the expression of sulfate reduction genes was considered in the Rex mutant, in order to determine whether the electrons may be forced internally (i.e. away from thiosulfate reduction processes) in the Rex mutant strain as compared to the parental strain. The deletion of rex increased expression for those genes that code for proteins associated with the early steps in sulfate reduction, specifically qmo $A B C D$ (Kuehl et al. 2014) and sat (Table 4-4). Interestingly, these were the same genes that were decreased in the parental strain when grown with thiosulfate. Therefore, the deletion of rex most likely disrupted the proper electron flow for thiosulfate reduction to proceed. Specifically, the Rex mutant resembled a sulfate-starved state so gene expression is altered to increase sulfate reduction, which was inhibitory when thiosulfate was the sole electron acceptor.

To begin to determine the mechanism for thiosulfate reduction in $\mathrm{DvH}$ and how Rex disrupts this mechanism, thiosulfate metabolism was considered further. The reduction of thiosulfate is endergonic and therefore, must be coupled to a more exergonic process in order to convert energy (Stoffels et al. 2012). One possible mechanism would be to link the reduction of thiosulfate to the proton motive force, as was shown for PhsABC from $S$. enterica (Stoffels et al. 2012). This enzyme is similar to the more familiar formate dehydrogenase (FhnGHI) from E. coli (Jormakka et al. 2002), except, for PhsABC the proton motive force is used to drive thiosulfate reduction instead of adding to the proton gradient. Because of this, thiosulfate reduction is presumed to occur at the periplasm in $\mathrm{DvH}$, and linked to the periplasmic membrane with the electrons being supplied by the menaquinone pool (Stoffels et al. 2012; Hocking et al. 2014).

In $\mathrm{DvH}$, the genes annotated as $p h s A B$ have high protein identity with PhsAB as expected (PhsA: 32\% identity, BLAST probability 3e-112, 94\% coverage; PhsB $72 \%$ identity, BLAST probability 3e-29, 43\% coverage); however, $p h s C$ has not been annotated in DvH. 
Additionally, a much smaller enzyme, purified from cell extract, was shown to reduce thiosulfate, but required a hydrogenase and methyl viologen as an electron carrier to facilitate thiosulfate reduction in vitro (Haschke et al. 1971). Therefore, the specific membrane component $(\mathrm{PhsC})$ is not currently known for $\mathrm{DvH}$. The role of PhsAB was examined for its role in the reduction of thiosulfate and its connection to Rex.

Interestingly, $p h s A B$ is thought to be controlled by Rex in two Desulfovibrionales, $D$. alaskensis G20 and D. vulgaris str. Miyazaki F, but not DvH, based on an identified Rexbinding site upstream of the encoding operons (Ravcheev et al. 2012). However, transcript studies examining a Rex mutant in D. alaskensis G20 (Kuehl et al. 2014) and DvH grown by sulfate respiration with lactate compared to the parental strain showed no change (cutoff being twofold) in expression of $p h s A B$, which was interpreted here to mean that Rex does not control $p h s A B$ expression. A transposon mutant for $p h s A$ was grown on different substrates (Figure 4-3). As predicted, the PhsA mutant was impaired for growth when respiring thiosulfate with lactate as compared to the wild-type DvH strain, although this strain was unaffected when pyruvate was added instead of lactate,. The deletion of $p h s A \operatorname{did}$ not prevent growth on thiosulfate and $p h s A B$ transcript is much lower in concentration ( $\sim 15-100$-fold less) than the genes that code for proteins responsible for sulfate reduction processes (Table 4-8). This was interpreted to mean that additional thiosulfate reductases are encoded in the genome, and the observed differences between the Rex mutant and parental strain grown by respiration with thiosulfate were a growth effect and not due to the regulation of $p h s A B$ by Rex.

Further examination of the genome of $\mathrm{DvH}$ revealed another possible thiosulfate reductase, DVU3143-5, which did increase in expression when DvH was respiring thiosulfate with pyruvate (Table 4-8). DVU3143 is annotated as an iron-sulfur cluster 
binding protein that resembles PhsB (30\% identity, BLAST probability 3e-16, 35\%

coverage). DVU3144 is annotated as a cytochrome c family protein that poorly resembles PhsA. And DVU3145 is annotated as a b-type hydrogenase (periplasmic) that resembles PhsC (34\% identity, BLAST probability 1e-21, 96\% coverage). Additionally, DVU3143 and DVU3144 appeared to have a periplasmic membrane localization signal as would be predicted for these two components (SignalP 4.1 server, data not shown) (Petersen et al. 2011). Therefore, we predict DVU3143-5 to be a periplasmic-facing membrane-bound thiosulfate reductase that is linked to the proton motive force. Characterization of DVU3143-5 is currently in progress.

In Desulfovibrio gigas, flavoredoxin (Flr) is required to couple the oxidation of hydrogen to thiosulfate reduction within the cytoplasm, and the expression of flr was shown by northern blot to increase in the presence of sulfite or thiosulfate, but not sulfate (Broco et al. 2005). Because a Flr mutant was impaired for growth on sulfite as well as thiosulfate, thiosulfate was concluded to be a product of sulfite reduction, giving credence to sulfite reduction by intermediates. Specifically, trithionate and thiosulfate were formed, each occurring by a two-electron transfer reaction (Kobayashi et al. 1969; Broco et al. 2005). Furthermore, the presence of sulfite was shown to inhibit the reduction of trithionate or thiosulfate although the mechanism for this inhibition is unknown. Once sulfite is depleted from the culture trithionate or thiosulfate were reduced as observed by depletion of these intermediates and formation of sulfide (Broco et al. 2005). In DvH, flr did not increase in expression regardless of the electron acceptor (Dehal et al. 2010), and Rex did not appear to control the expression of flr in D. alaskensis G20 (Kuehl et al. 2014). Additionally, the Rex $x_{\text {DvH }}$ mutant resembles an electron starved state which should be similar to the build-up of thiosulfate within the cytoplasm and be similar to a Flr mutant. However, a Flr mutant in 
DvH did not appear to be impaired for growth in sulfate, sulfite, or thiosulfate as compared to the parental strain (data not shown). This was interpreted to mean that the role Flr plays in DvH may be different from what was observed in D. gigas.

From these findings we present a new model for thiosulfate metabolism in DvH (Figure 4-4) and the role of Rex in thiosulfate reduction. In this model, DVU3143-5 is the thiosulfate reductase that is coupled to the proton motive force, although PhsAB is still functioning in the periplasm to generate sulfite for subsequent reduction in the cytoplasm. DVU3143-5 is presumed to receive electrons from the menaquinone pool, most likely supplied by QrcABCD and is in competition with QmoABC that supplies electrons to APS reductase for sulfate reduction. To substantiate this, the deletion of $\operatorname{qr} A B C D$ impairs growth on thiosulfate, suggesting its role in thiosulfate reduction (data not shown). However, the QrcABCD mutant after an extended lag is able to grow, although at a slower rate, which was interpreted to mean that electrons may be supplied to DVU3143-5 from an alternate source as well. Ldh was proposed to provide electrons to DVU3143-5 in a similar manner as it does to QmoABC. Furthermore, DVU3145 was predicted to resemble a hydrogenase, which may allow for electrons to be transferred directly to this complex from hydrogen. Interestingly, during thiosulfate reduction, $p f l$ and $f d h A B$ increase in expression, which was interpreted to mean that formate is produced in greater quantity in the cytoplasm from pyruvate. Subsequently formate would be transported to the periplasm, where it is oxidized to protons and $\mathrm{CO}_{2}$, with the electrons being transferred to TpI- $\mathrm{c}_{3}$. This would allow for an electron circuit that is shorter and more direct, and avoid transferring electrons to ferredoxin which is associated with sulfate-reduction processes within the cytoplasm. Additionally, the hydrogenases in the periplasm are also decreased in the presence of thiosulfate, which may allow for DVU3143-5 to oxidize the hydrogen instead. Also, TpIc3 may be more likely to 
target QrcABCD at the periplasmic membrane with the other hydrogenases being decreased in abundance. Therefore, in a strain deleted for rex, we hypthesize that the increased expression of qmo $A B C$ may funnel the electrons towards sulfate reduction and away from DVU3143-5, limiting thiosulfate reduction. Thus, the role of Rex was determined to maintain steady-state sulfate reduction levels, and when sulfate reduction is slowed, i.e. an increase in $\mathrm{NADH} / \mathrm{NAD}^{+}$ratio, Rex de-represses genes that code for proteins involved with funneling the electrons to sulfate reduction processes, which may also include scavenging mechanisms for sulfate, in order to restore $\mathrm{NADH} / \mathrm{NAD}^{+}$levels. Consequently, as thiosulfate reduction does not occur within the cytoplasm the Rex mutant was impaired for growth when respiring thiosulfate regardless of the electron donor. 
Chapter 5: $\quad$ Supplementary projects and discussion 


\section{Section 5.1. Overview}

In this section, additional projects not directly discussed in earlier chapters are provided. This chapter includes: requirement of yeast extract in fermenting cultures, northern blot analysis of aps $A$, Rex mutant sensitivity to molybdate, examination of additional regulators of sulfate reduction (e.g. HcpR and LysX), and additional details of several protocols (qPCR, RACE, NADH quantification).

\section{Section 5. 2. Yeast extract required for DvH to ferment pyruvate efficiently}

DvH does not grow appreciably in pyruvate fermenting medium as observed spectrophotometrically by a low optical density $(\mathrm{OD} 600<0.1)$. This low yield poses a challenge for several of the analyses to be performed in this study, where cell number and growth are essential. In our laboratory, addition of yeast extract at $0.1 \%$ (wt/vol) and/or cysteine $(0.5 \mathrm{mM})$ to the media have been shown to assist in the growth of DvH by fermentation. Because the work planned was focused on respiration and fermentation processes specific to the sulfate reduction pathway, it was essential to make sure that any differences being observed were not related to the addition of yeast extract or cysteine to the fermenting medium. Therefore, JW710 (the parental strain used to construct any of the MED strains) was grown fermentatively with pyruvate, with yeast extract, with cysteine, or with all components, in triplicate. After 73 hours cultures were assessed for protein and sat expression (Figure 5-1). JW710 grew to a higher OD600 max $_{\text {and }}$ and higher total protein content when both yeast extract and cysteine were added. The addition of both yeast extract and cysteine allowed for increased cell density and protein, while the addition of either one alone had slightly increased or no affect at all, respectively. Furthermore, when sat transcript is 
normalized to total protein the abundance of sat is consistent across all samples. Therefore, addition of yeast extract at $1 \% \mathrm{wt} / \mathrm{vol}$ and cysteine at $0.5 \mathrm{mM}$ should not interfere with the studies planned.
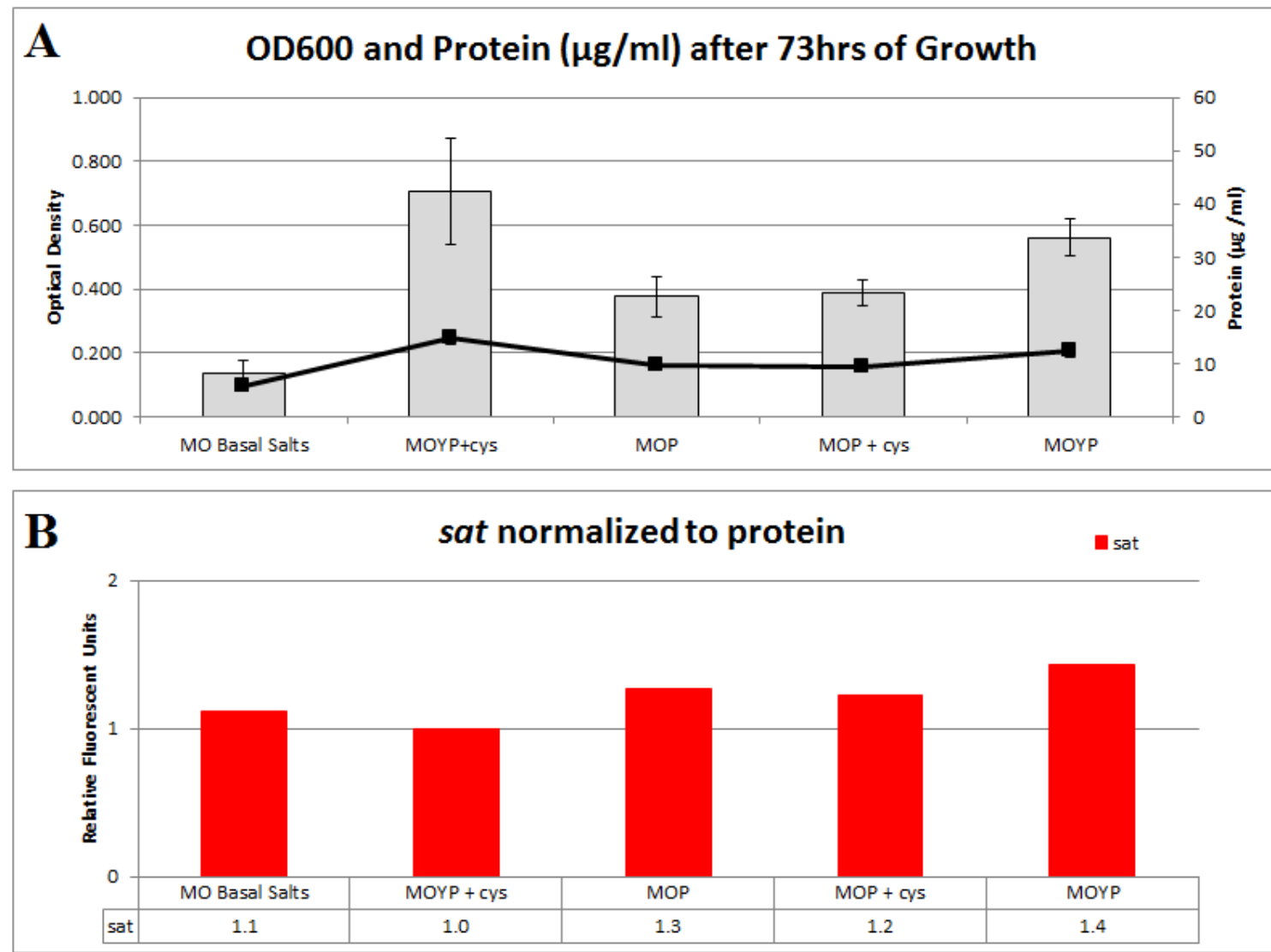

Figure 5-1: Pyruvate fermentation control experiment. JW710 was grown fermentatively for 73 hours in MO basal salts supplemented with either Pyruvate (P), Yeast Extract (Y), Cysteine (cys) or all three. A) Optical density after 73 hours was determined and samples were collected and assayed for total protein. B) sat transcript was determined and normalized to protein, and then normalized to JW710 MOYP + cys. 


\section{Section 5. 3. Northern blot analysis}

Initial examination of transcript analysis for $a p s B$ was determined by northern blot.

DvH was grown and mRNA isolated according to protocols discussed in the methods section (Zane et al. 2010). The cells were cultured in lactate (60 mM) respiring medium with either sulfate $(30 \mathrm{mM})$ or sulfite $(20 \mathrm{mM})$ as the terminal electron acceptor. Approximately $5 \mu \mathrm{g}$ of total RNA was electrophoresed (90 min at $75 \mathrm{~V}$ ) through a $1.5 \%$ (wt/vol) agarose gel followed by transfer to a positively charged membrane (Figure 5-2). apsB $A$ operon was determined to be expressed at a higher level when $\mathrm{DvH}$ was grown in sulfate compared with sulfite as the terminal electron acceptor confirming previous studies (Zane et al. 2010).

$\operatorname{aps} B A(\sim 2500$ bases $)$

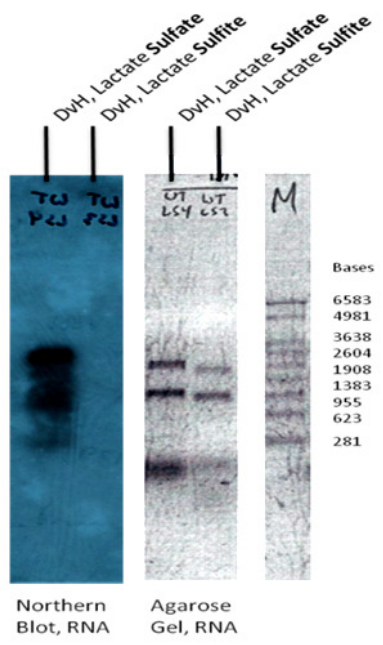

Figure 5-2: Northern blot analysis of aps $A$. DvH strain probed for aps $A$-containing transcript. Determination of aps $A$ expression in RNA samples isolated from DvH grown by sulfate respiration with lactate or sulfite as terminal electron acceptor. RNA was probed with a DNA fragment of apsB. X-ray film (LEFT), denaturing agarose gel (Middle) are shown. 


\section{Section 5. 4. Rex mutant is sensitive to molybdate}

SRB, including DvH, are sensitive to high concentrations of molybdate, although trace amounts are required for growth (Biswas et al. 2009). Molybdate has a similar structure as sulfate and competes with sulfate for the active site of Sat. However, the complex formed by Sat with molybdate is unstable and an ATP molecule would be squandered. Because of the competition between sulfate and molybdate for Sat the cell cannot sustain growth (Peck 1959; 1962). Since a deletion in rex was predicted to increase sat expression, and therefore Sat, it was assumed that this strain should be sensitive to molybdate. The Rex marker-exchange deletion was assayed for molybdate sensitivity, and as expected the Rex mutant was inhibited for growth compared with the parental strain on sub-lethal concentrations of molybdate (Figure 5-3). The interpretation of these results was that energy was consumed but an electron acceptor was not generated.

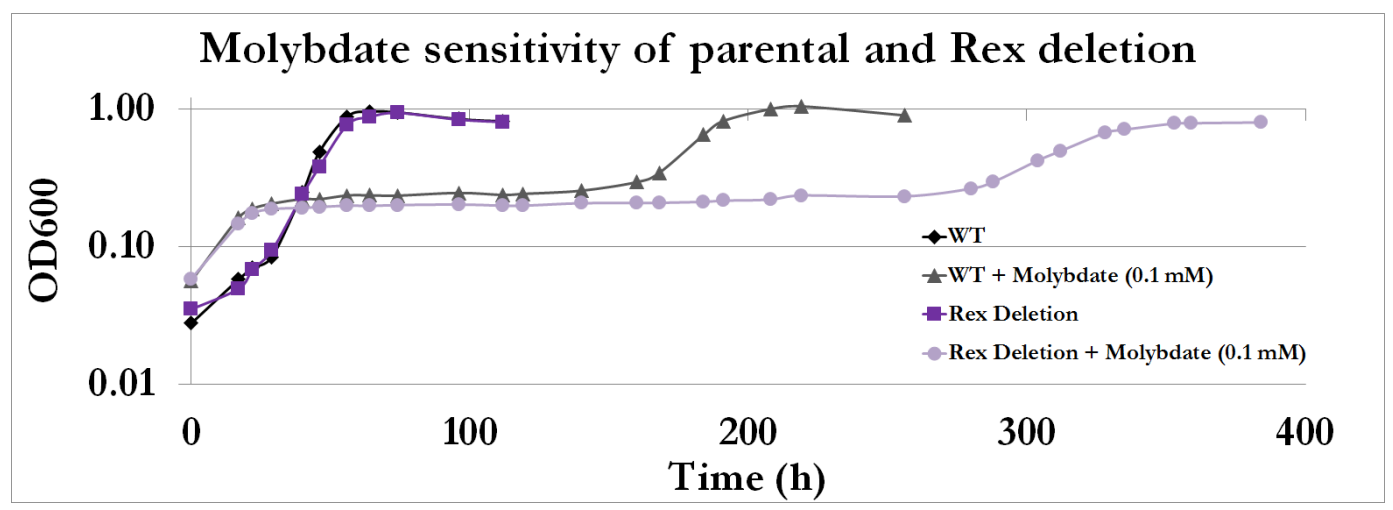

Figure 5-3: Rex mutant is more sensitive to molybdate than its parental strain. Parental and Rex mutant grown in defined MOLS4 $(\mathrm{n}=3)$. Addition of Molybdate to a final concentration of 0 and $0.1 \mathrm{mM}$. 


\section{Section 5. 5. Other regulators predicted to control sulfate}

\section{reduction}

\section{Section 5. 5. 1. Overview}

From the work performed on $\operatorname{Rex}_{\mathrm{DvH}}$, there are certainly other components involved in regulating the sulfate reduction pathway, several of which have been postulated (Table 5-1) (Rodionov et al. 2004; Turkarslan et al. 2013; Christensen et al. 2014). The majority of these regulators are likely to control gene expression through their interaction with DNA.

Table 5-1: Potential regulators of sulfate reduction

\begin{tabular}{|c|l|c|c|}
\hline DVU & \multicolumn{1}{|c|}{ Name } & Strain identifier & $\begin{array}{c}\text { Gene Fitness* } \\
\left(\log _{2}\right)\end{array}$ \\
\hline DVU0057 & Transcriptional regulator, TetR family & GZ7888 & 0.86 \\
\hline DVU0744 & Sigma-54 dependent transcriptional regulator & GZ12635 & -0.05 \\
\hline DVU0916 & Rex, transcriptional regulator & JW3311, JW3319 & -2.86 \\
\hline DVU1142 & Transcriptional regulator, putative & GZ4443 & 0.44 \\
\hline DVU2275 & Sigma-54 dependent transcriptional regulator & GZ5190 & -0.04 \\
\hline DVU2547 & Transcriptional regulator, putative, HcpR family & JW9011 & 0.47 \\
\hline DVU2567 & Regulator for lysine biosynthesis and transport & GZ0481 & 0.28 \\
\hline DVU2690 & Hypothetical protein & GZ1801 & 0.56 \\
\hline DVU2799 & Transcriptional regulator, MarR family & GZ12386 & -0.71 \\
\hline DVU2802 & Transcriptional regulator, GntR family & GZ12985 & 0.92 \\
\hline
\end{tabular}

*Gene fitness values from DvH_MOLS4 experiment

Gene fitness across the genome for DvH grown on minimal medium containing lactate and sulfate has been determined (Fels et al. 2013). Examination of gene fitness for these predicted regulators revealed that the interruption of an individual gene correlated to a minor fitness advantage (positive) or defect (negative) compared to the parental strain (Table 5-1). The contribution of these potential regulators is less significant than what had been observed for rex, with a fitness defect $\left(\log _{2}=-2.86\right)$. Additionally, in the DvH transposon library rex was not obtained but all of the potential regulators mentioned above were.

Therefore, if these regulators are involved with the regulation of sulfate reduction they have minor roles or are compensated elsewhere. 


\section{Section 5. 5. 2. Transcript expression results for potential regulators}

Expression of sulfate reduction genes is known to decrease when sulfite is provided as the terminal electron acceptor as compared with sulfate (Wall et al. 2008; Zane et al. 2010). The mechanism for this is unknown. Recently, several regulators have been predicted to control sulfate reduction (Table 5-1), potentially by altering the expression of $s a t$ and aps $A$. It was interpreted that the change in abundance of the encoded proteins would alter the flux of electrons through this metabolic pathway (i.e. more transcript and so more protein would increase sulfate reduction processes). To determine the role of these potential regulators, qRT-PCR was performed on parental DvH cells, on a marker-exchange deletion of $h c p R$, and on individual transposon insertion mutants of the regulators mentioned above, in defined lactate $(60 \mathrm{mM})$ medium with either sulfate $(30 \mathrm{mM})$ or sulfite $(20 \mathrm{mM})$ as the terminal electron acceptor (Table 5-2) (Table 5-3). Expression of sat, aps $A$ and $d s r A$ was determined. No observable difference greater than twofold was found among the majority of the strains, for the conditions assayed. Although, the hypothetical gene DVU2690 and sigma-54 transcription regulator DVU0744 did appear to have a slight loss of regulation control (i.e. sat did not decrease to the same degree as the parental strain with sulfite compared to sulfate). The annotated transcription regulators DVU0744, DVU2802 and DVU2275 and their role in sulfate reduction regulation are examined elsewhere (Turkarslan et al. 2013) (Serdar Turkarslan, personal communication). Overall, this preliminary study would suggest that several of these candidates seem likely to control gene expression and should be pursued in greater detail for their role in sulfate reduction regulation. 
Table 5-2: Sample collection and transcript analysis of potential regulators

\begin{tabular}{|c|c|c|c|c|c|c|c|c|}
\hline \multirow[b]{2}{*}{ Strain } & \multirow[b]{2}{*}{ Name } & \multirow[b]{2}{*}{ Media } & \multirow[b]{2}{*}{$\begin{array}{c}\text { OD600 } \\
\text { collected }\end{array}$} & \multicolumn{5}{|c|}{ Mean $\mathrm{Cq}^{\mathrm{a}}$} \\
\hline & & & & rpls & $r p m C$ & sat & $\operatorname{aps} A$ & $d s r A$ \\
\hline \multirow{2}{*}{$\mathrm{DvH}^{*}$} & \multirow{2}{*}{ wild-type } & LS4 & 0.455 & $22.62 \pm 0.03$ & $23.99 \pm 0.02$ & $22.73 \pm 0.02$ & $23.62 \pm 0.01$ & $21.68 \pm 0.02$ \\
\hline & & LS3 & 0.414 & $20.88 \pm 0.06$ & $23.61 \pm 0.03$ & $22.65 \pm 0.06$ & $24.71 \pm 0.06$ & $22.40 \pm 0.03$ \\
\hline \multirow{2}{*}{ GZ0481" } & \multirow{2}{*}{ bys $X$} & LS4 & 0.249 & $21.85 \pm 0.00$ & $24.44 \pm 0.03$ & $23.51 \pm 0.02$ & $24.57 \pm 0.00$ & $22.22 \pm 0.02$ \\
\hline & & LS3 & 0.228 & $20.03 \pm 0.05$ & $23.08 \pm 0.04$ & $24.65 \pm 0.01$ & $26.48 \pm 0.00$ & $21.11 \pm 0.05$ \\
\hline \multirow{2}{*}{ GZ12635* } & \multirow{2}{*}{ DVU0744 } & LS4 & 0.320 & $21.89 \pm 0.01$ & $23.76 \pm 0.02$ & $22.83 \pm 0.03$ & $23.32 \pm 0.01$ & $21.67 \pm 0.03$ \\
\hline & & LS3 & 0.421 & $20.93 \pm 0.02$ & $23.92 \pm 0.07$ & $22.95 \pm 0.04$ & $24.61 \pm 0.01$ & $22.05 \pm 0.01$ \\
\hline \multirow{2}{*}{ GZ7888* } & \multirow{2}{*}{ DVU0057 } & LS4 & 0.425 & $22.19 \pm 0.03$ & $23.91 \pm 0.03$ & $22.64 \pm 0.05$ & $23.28 \pm 0.05$ & $21.74 \pm 0.03$ \\
\hline & & LS3 & 0.420 & $20.72 \pm 0.02$ & $23.38 \pm 0.03$ & $23.74 \pm 0.05$ & $25.43 \pm 0.04$ & $21.69 \pm 0.06$ \\
\hline GZ5190* & DVU2275 & LS4 & 0.380 & $21.43 \pm 0.05$ & $23.67 \pm 0.03$ & $22.21 \pm 0.05$ & $23.05 \pm 0.01$ & $21.19 \pm 0.02$ \\
\hline \multirow{2}{*}{ GZ12985* } & \multirow{2}{*}{ DUV2802 } & LS4 & 0.435 & $21.22 \pm 0.01$ & $22.84 \pm 0.04$ & $21.87 \pm 0.03$ & $22.50 \pm 0.01$ & $20.93 \pm 0.01$ \\
\hline & & LS3 & 0.451 & $20.64 \pm 0.05$ & $22.75 \pm 0.01$ & $22.43 \pm 0.05$ & $24.55 \pm 0.01$ & $20.64 \pm 0.01$ \\
\hline \multirow{2}{*}{ GZ4443* } & \multirow{2}{*}{ DVU1142 } & LS4 & 0.410 & $21.86 \pm 0.02$ & $23.61 \pm 0.01$ & $22.63 \pm 0.01$ & $23.42 \pm 0.03$ & $21.63 \pm 0.02$ \\
\hline & & LS3 & 0.495 & $20.88 \pm 0.02$ & $22.79 \pm 0.04$ & $22.76 \pm 0.02$ & $25.10 \pm 0.02$ & $20.98 \pm 0.02$ \\
\hline \multirow{2}{*}{ JW710"\# } & \multirow{2}{*}{ parental } & LS4 & 0.257 & $20.49 \pm 0.02$ & $23.70 \pm 0.01$ & $21.62 \pm 0.01$ & $22.72 \pm 0.03$ & $21.84 \pm 0.04$ \\
\hline & & LS3 & 0.243 & $19.31 \pm 0.06$ & $22.11 \pm 0.03$ & $24.46 \pm 0.04$ & $25.48 \pm 0.02$ & $20.16 \pm 0.03$ \\
\hline \multirow{2}{*}{ JW9011 } & \multirow{2}{*}{$\Delta h c p R$} & LS4 & 0.236 & $21.96 \pm 0.03$ & $24.03 \pm 0.02$ & $22.74 \pm 0.00$ & $23.90 \pm 0.01$ & $22.29 \pm 0.04$ \\
\hline & & LS3 & 0.261 & $19.78 \pm 0.05$ & $22.90 \pm 0.05$ & $25.54 \pm 0.01$ & $26.66 \pm 0.02$ & $21.03 \pm 0.02$ \\
\hline
\end{tabular}

Refer to Table 5-3 for descriptive legend. 
Table 5-3: Transcript analysis of potential regulators

\begin{tabular}{|c|c|c|c|c|}
\hline \multirow[b]{2}{*}{ Name } & \multirow[b]{2}{*}{ Media } & \multicolumn{3}{|c|}{ Transcript $^{a}$} \\
\hline & & sat & $\operatorname{aps} A$ & $d s r A$ \\
\hline \multirow{2}{*}{${ }^{*}$ wild-type } & LS4 & $1.00 \pm 0.02$ & $1.00 \pm 0.01$ & $1.00 \pm 0.01$ \\
\hline & LS3 & $0.52 \pm 0.02$ & $0.24 \pm 0.01$ & $0.60 \pm 0.01$ \\
\hline \multirow{2}{*}{${ }^{\#}$ lys $X$ transposon } & LS4 & $1.00 \pm 0.02$ & $1.00 \pm 0.01$ & $1.00 \pm 0.01$ \\
\hline & LS3 & $0.16 \pm 0.00$ & $0.10 \pm 0.00$ & $0.71 \pm 0.03$ \\
\hline \multirow{2}{*}{${ }^{*}$ DVU2690 transposon } & LS4 & $1.00 \pm 0.02$ & $1.00 \pm 0.01$ & $1.00 \pm 0.04$ \\
\hline & LS3 & $0.78 \pm 0.02$ & $0.43 \pm 0.01$ & $0.69 \pm 0.01$ \\
\hline \multirow{2}{*}{ *DVU0744 transposon } & LS4 & $1.00 \pm 0.02$ & $1.00 \pm 0.01$ & $1.00 \pm 0.03$ \\
\hline & LS3 & $0.71 \pm 0.03$ & $0.32 \pm 0.01$ & $0.59 \pm 0.01$ \\
\hline \multirow{2}{*}{ *DVU0057transposon } & LS4 & $1.00 \pm 0.04$ & $1.00 \pm 0.04$ & $1.00 \pm 0.02$ \\
\hline & LS3 & $0.24 \pm 0.01$ & $0.12 \pm 0.00$ & $0.53 \pm 0.02$ \\
\hline \multirow{2}{*}{ *DVU2275 transposon } & LS4 & $1.00 \pm 0.04$ & $1.00 \pm 0.02$ & $1.00 \pm 0.03$ \\
\hline & LS3 & $0.51 \pm 0.01$ & $0.27 \pm 0.01$ & $0.50 \pm 0.01$ \\
\hline \multirow{2}{*}{${ }^{*}$ DVU2799 transposon } & LS4 & $1.00 \pm 0.02$ & $1.00 \pm 0.02$ & $1.00 \pm 0.02$ \\
\hline & LS3 & $0.45 \pm 0.01$ & $0.23 \pm 0.01$ & $0.52 \pm 0.01$ \\
\hline \multirow{2}{*}{ *DUV2802 transposon } & LS4 & $1.00 \pm 0.03$ & $1.00 \pm 0.02$ & $1.00 \pm 0.02$ \\
\hline & LS3 & $0.54 \pm 0.02$ & $0.20 \pm 0.00$ & $0.61 \pm 0.01$ \\
\hline \multirow{2}{*}{${ }^{*}$ DVU1142 transposon } & LS4 & $1.00 \pm 0.01$ & $1.00 \pm 0.02$ & $1.00 \pm 0.02$ \\
\hline & LS3 & $0.50 \pm 0.01$ & $0.18 \pm 0.00$ & $0.86 \pm 0.02$ \\
\hline \multirow{2}{*}{${ }^{\#}$ parental } & LS4 & $1.00 \pm 0.01$ & $1.00 \pm 0.02$ & $1.00 \pm 0.03$ \\
\hline & LS3 & $0.06 \pm 0.00$ & $0.07 \pm 0.00$ & $1.18 \pm 0.03$ \\
\hline \multirow{2}{*}{${ }^{\#} \Delta h c p R$} & LS4 & $1.00 \pm 0.01$ & $1.00 \pm 0.01$ & $1.00 \pm 0.03$ \\
\hline & LS3 & $0.05 \pm 0.00$ & $0.06 \pm 0.00$ & $0.75 \pm 0.02$ \\
\hline
\end{tabular}

These results include two experiments that were performed at two separate times. *denotes experiment 1 , while \# denotes experiment 2 . OD600 was monitored through growth and samples were collected for analysis at mid-exponential (experiment 1) or at early-exponential (experiment 2). Approximately $100 \mathrm{ng}$ of TURBO ${ }^{\mathrm{TM}}$ DNasetreated RNA was converted to cDNA and $1 \mu \mathrm{L}$ of cDNA (5 ng of RNA) was used per qRT-PCR. Critical threshold $(\mathrm{Cq})$ is presented for all genes tested, including two reference genes, $r p l S$ and $r p m C$. For experiment 1, the efficiency for each gene was determined: $r p l S=95.2 \%, r p m C=91.2 \%, s a t=100.5 \%$, aps $A=95.0 \%$ and $d s r A=$ $98.6 \%$; and for experiment 2: $r p l S=94.7 \%, r p m C=89.6 \%$, sat $=100.3 \%$, aps $A=$ $92.0 \%$ and $d s r A=89.0 \%$. ${ }^{a}$ Critical threshold $(\mathrm{Cq})$ for specified gene, with technical error determined as standard error of the mean $(n=3)$. 


\section{Section 5. 5. 3. qPCR primer design strategy}

For every gene analyzed, two optimization steps were taken for primer design to ensure quality of transcript data. First, three forward and three reverse primers were designed with specified parameters (i.e. $\mathrm{T}_{\mathrm{m}}$ and length). Then, each combination (e.g. Forward primer 1 with Reverse primer 1), for a total of nine, were used to amplify the target from 10 ng of gDNA template. The quality of each primer set was then determined (Figure 5-4) and two with reasonable amplification curves, including no amplification of the no-template controls (NTC) and having a single peak in the melt curve, are then used to determine efficiency (Figure 5-5). The efficiency of a primer set is determined by amplifying a dilution series of template DNA (or RNA, in the form of cDNA), typically starting from a $100 \mathrm{ng} / \mu \mathrm{l} \mathrm{stock}$, and solving for the curve generated from plotting Ct versus concentration. Acceptable primer sets for a 10-fold dilution series would have a slope $(\mathrm{m})$ of $\sim-3.4, \mathrm{R}^{2}>0.990$ and efficiency $80-110 \%$. For each gene the one primer set determined to fulfill these requirements was used. A list for all optimized perimer sets is provided (Table 7-5). 


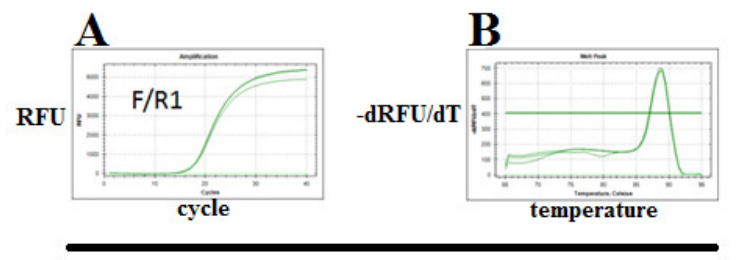

"GOOD"
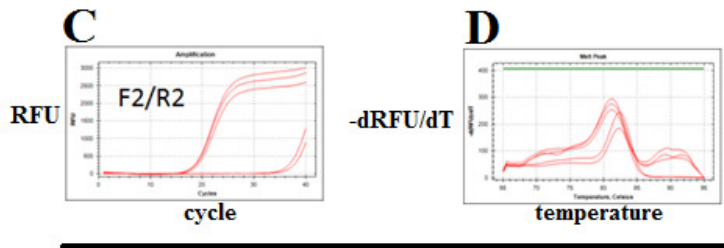

"BAD"

Figure 5-4: Primer optimization results of a "good" and "bad" primer set. Graphs A and C are amplification curves (RFU vs Cycle) and graphs B and $\mathrm{D}$ are melt curves (dRFU/dT vs temperature) of two separate primers sets (F/R1 vs F2/R2), of the nine that were performed, that have been amplified from the same gDNA template. In the "bad" example, fluorescence was detected in the no template control (NTC) in graph C, and in the melt curve (D) multiple peaks (and shouldered curves) were seen as a result of the NTC amplification (i.e. primer dimers).

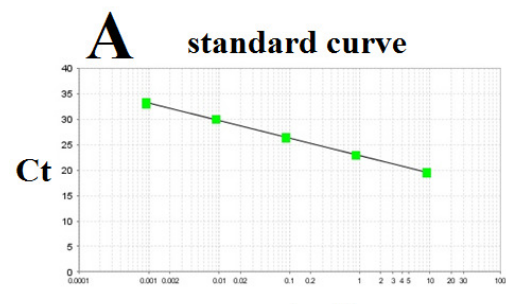

concentration

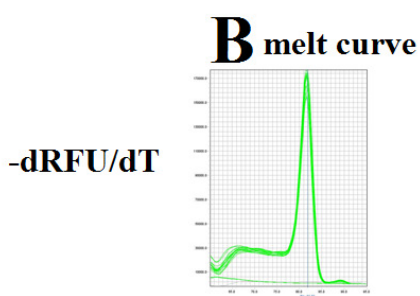

temperature

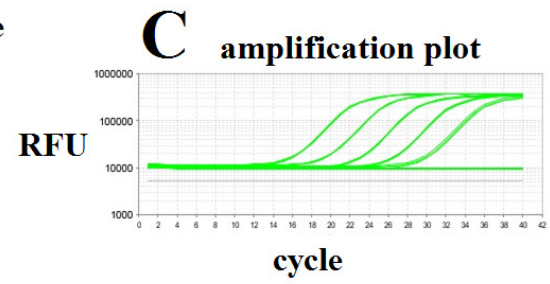

Figure 5-5: Primer efficiency determination for aps $\boldsymbol{A}$. gDNA used as template for standard curve (100 ng-10 pg, 10-fold dilution). A) Standard curve plot for efficiency determination (Ct vs template concentration); B) melt curve (dRFU/dT vs temperature); and C) Amplification Plot (Semi-log) (RFU vs Cycle). 


\section{Section 5. 6. Additional RACE protocol and discussion}

\section{Section 5. 6. 1. Overview}

In the case that the strategy described in the methods section did not work (Section 7.9)

(Figure 7-6), i.e. poor sequence reads or no single fragment, a new approach was developed.

The modifications to the protocol included modified primers that contained overhangs for cloning purposes via SLIC (Li et al. 2007), and additional components for stabilization of the desired product throughought many of the steps. As a proof of principle for the cloning procedure the TSS for sat was first determined for DvH grown by sulfate respiration with lactate. Indeed, the results for the TSS of sat confirmed previous results.

\section{Section 5. 6.2. Modifications to the TSS protocol}

RNA was isolated as described elsewhere. A 5- $\mu \mathrm{l}$ aliquot (20 ng/ $\mu \mathrm{l})$ of DNase-treated RNA was reverse transcribed to single stranded cDNA via iScript ${ }^{\mathrm{TM}}$ Select cDNA synthesis kit (Bio-Rad) with primer DVU1295-sat-GSP1, degraded with RNase A/T1 Mix and RNaseH (Thermo Fischer Scientific) according to manufacturers protocol. With terminal deoxynucleotidyl transferase (Thermo Fisher Scientific), an adenosine tail was added to the cDNA, followed by generation of the second DNA strand with iScript ${ }^{\mathrm{TM}}$ and primer RACE$2^{\text {nd }}$ Strand. dsDNA was purified, diluted 1:1000 and amplified by PCR to generate DNA fragment PCR1, with primers: DVU1295-GSP2 and AUAP. Thirty cycles of amplification were carried out $\left(30 \mathrm{sec}\right.$ at $94^{\circ} \mathrm{C}, 30 \mathrm{sec}$ at $58^{\circ} \mathrm{C}, 1 \mathrm{~min}$ at $\left.72^{\circ} \mathrm{C}\right)$ followed by a final extension for 5 min at $72^{\circ} \mathrm{C}$ with Taq DNA Polymerase (NEB). From this point on in the protocol the modifications were made. An additional PCR (PCR2) followed, which was the same protocol as PCR1, but with 1:1000 diluted PCR1 as template and nested primers (AUAP-cloning and DVU1295-GSP4-cloning). Following standard PCR protocols, an additional fragment 
(backbone fragment, "SpUC", 1885 bp) was amplified from pMO719 with primers SpecRpUC-F and SpecRpUC-R. This fragment included the spectinomycin resistance marker and pUC ori. The PCR2 fragments containing overhangs to the "SpUC" backbone fragment and "SpUC" backbone fragment were T4-treated and cloned via a standard SLIC protocol. A 5-, 50-, and 500- $\mu$ l aliquot of transformed cells were plated in LC-medium containing spectinomycin. Colony forming units were counted and PCR screened with primers pMO719-XbaI-dn and pBG1-2199-F for inserted segments (>200 bps). For correctly screened colonies, plasmid was purified, sequenced and mapped to the genome. Additional measures were taken, including addition of ectoine $(0.5 \mathrm{M})$ to the cDNA generation step or betaine $(1 \mathrm{M})$ or DMSO $(5 \% \mathrm{vol} / \mathrm{vol})$ throughout several of the PCR amplification steps (Shi et al. 2006), use of a more thermo-stable enzyme in the cDNA generation step (ThermoScript-RT), and modified PCR protocols (e.g. touchdown-PCR (Don et al. 1991)), which had previously been shown to improve these in vitro processes. An additional modification to this procedure, although not tested, could have been the use of a modified base, 7-deaza-2'-dGTP, which has been shown to alleviate some of the difficulties in polymerizing GC-rich regions (Hurt et al. 2012). 
Table 5-4: Primer list for RACE experiments (cloning method)

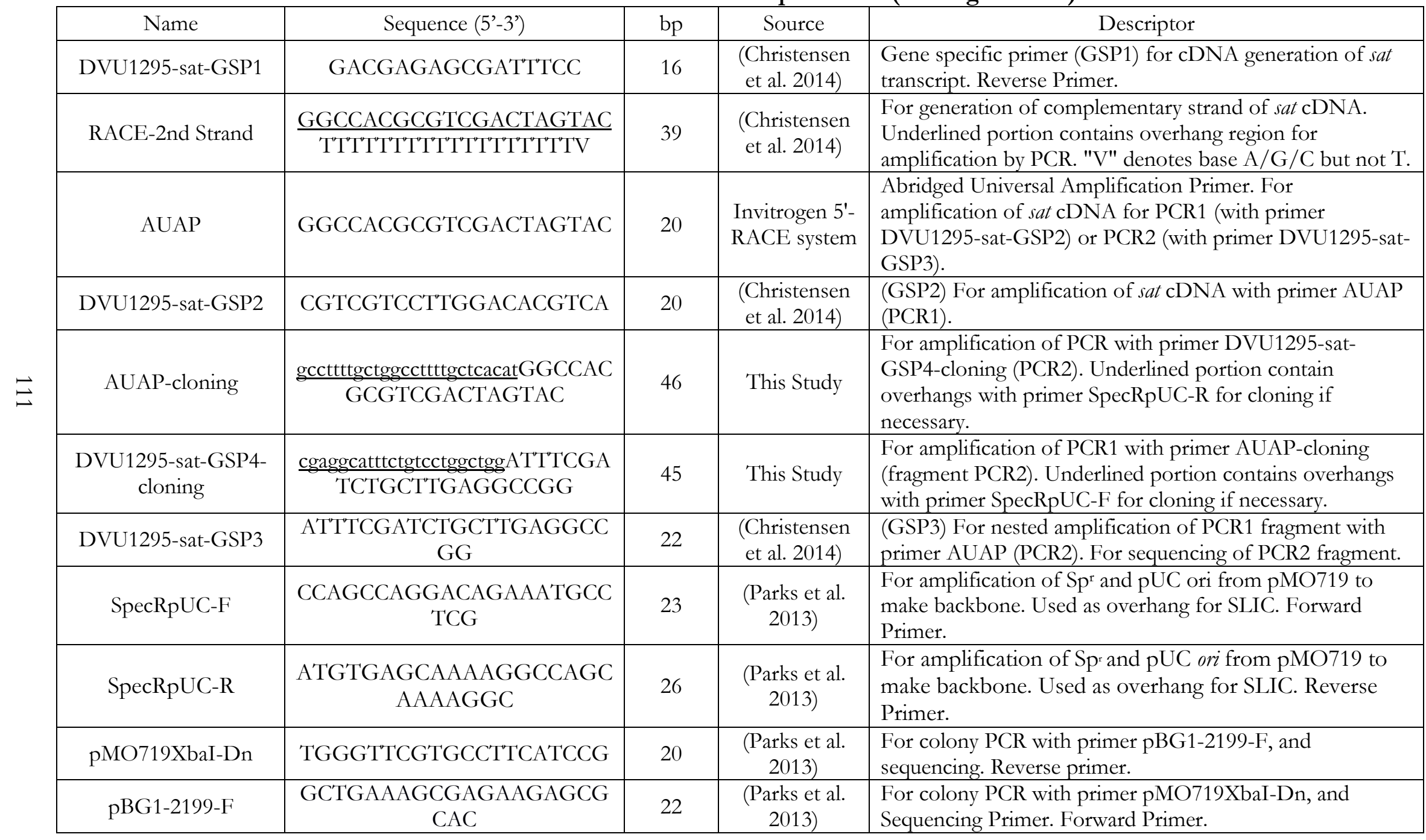




\section{Section 5. 7. NADH quantification discussion}

The specific method for NADH quantification is described elsewhere (Section 7.11), this section is designated for the discussion of the assay. After an initial attempt (data not shown), in which NADH was undetected, there was concern that the reduced form was not recovered due to initial purification procedures. In particular, the protocol called for a freeze/thaw cycle whereby an extended period of time would elapse and be exposed to oxygen. To determine whether NADH was being oxidized within the time-frame of the experiment, purified NADH $(1 \mathrm{mM})$ was added to several steps along the process and assayed for both $\mathrm{NAD}^{+}$and $\mathrm{NAD}_{\text {total }}$ in order to determine when NADH was "lost". As expected, the added NADH was only recovered in the "correct" reduced form when it was added after the desalting column step (Figure 5-6). Most likely, proteins capable of oxidizing NADH had been separated from the sample after the column purification. Therefore, this method would not allow determination of the ratio of the pyridine nucleotides from a cell as all $\mathrm{NADH}$ is converted to its oxidized form, $\mathrm{NAD}^{+}$, during the purification process. Additionally, to determine that the media used in the studies planned would not affect the signal, standards for $\mathrm{NAD}^{+}$and $\mathrm{NAD}_{\text {total }}$ at several concentrations were determined in solutions containing media components, including: MO basal salts, sulfide $(0.5 \mathrm{mM})$, cysteine $(0.5 \mathrm{mM})$, and thioglycolate $(1.2 \mathrm{mM})$. The results revealed that that sulfide and cysteine artificially increased the signal, while MO basal salts decreased the signal and thioglycolate did not affect the assay (Figure 5-7). Therefore, additional steps would be required to properly assess pyridine nucleotide concentrations. Specifically, a different analysis altogether, and not the colorimetric assay used in this study, would be required to obtain the desired results. Therefore, 
an assay that included mass spectrometry analysis (Sporty et al. 2008), tools our laboratory does not have immediate access too, may be necessary to determine $\mathrm{NAD}^{+}$and NADH conentrations.

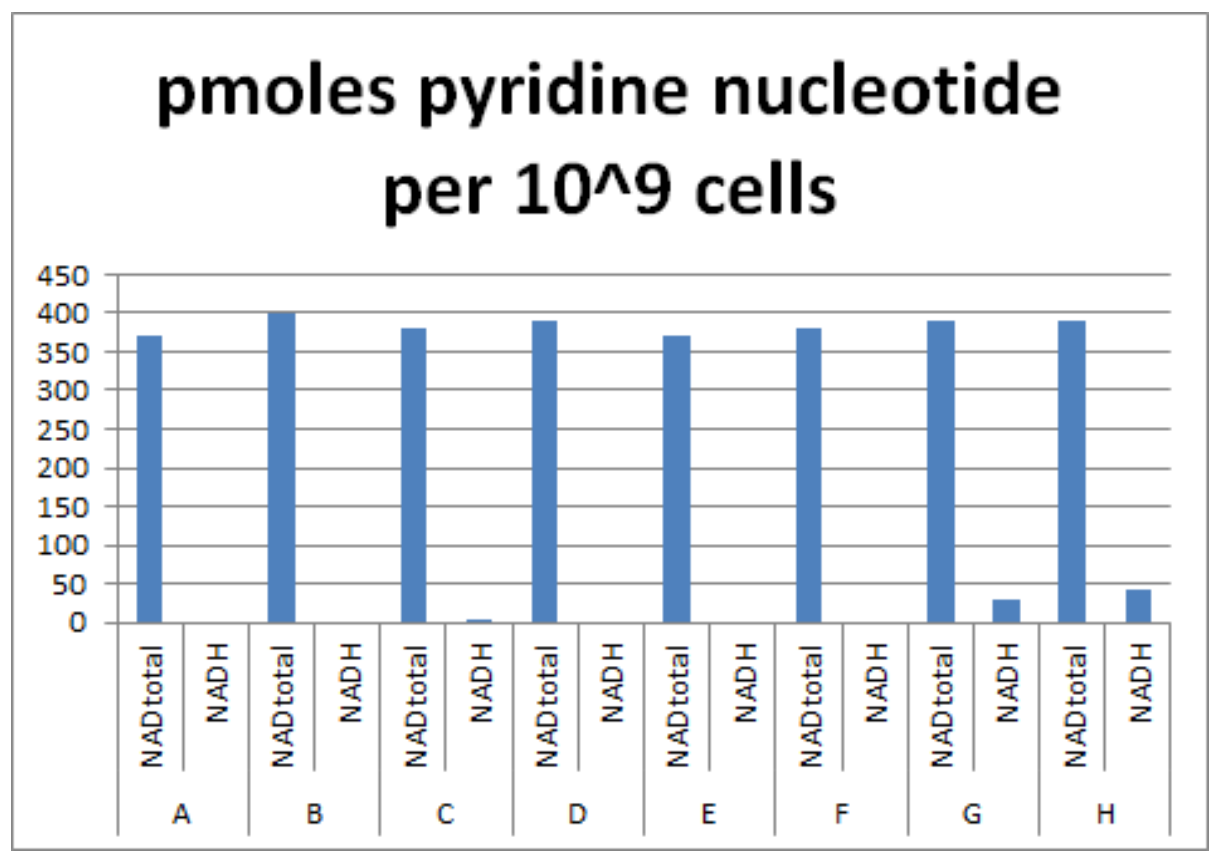

Figure 5-6: Pyridine quantification determination. Eight samples were run in parallel (A-H). NADH $(1 \mathrm{mM})$ was added to each of the samples along the purification process, except sample A (control). Sample $G$ is addition of NADH right after being passed through desalting column. Sample $H$ is sample $G$ that had been incubated an additional 12 hours on the benchtop. 


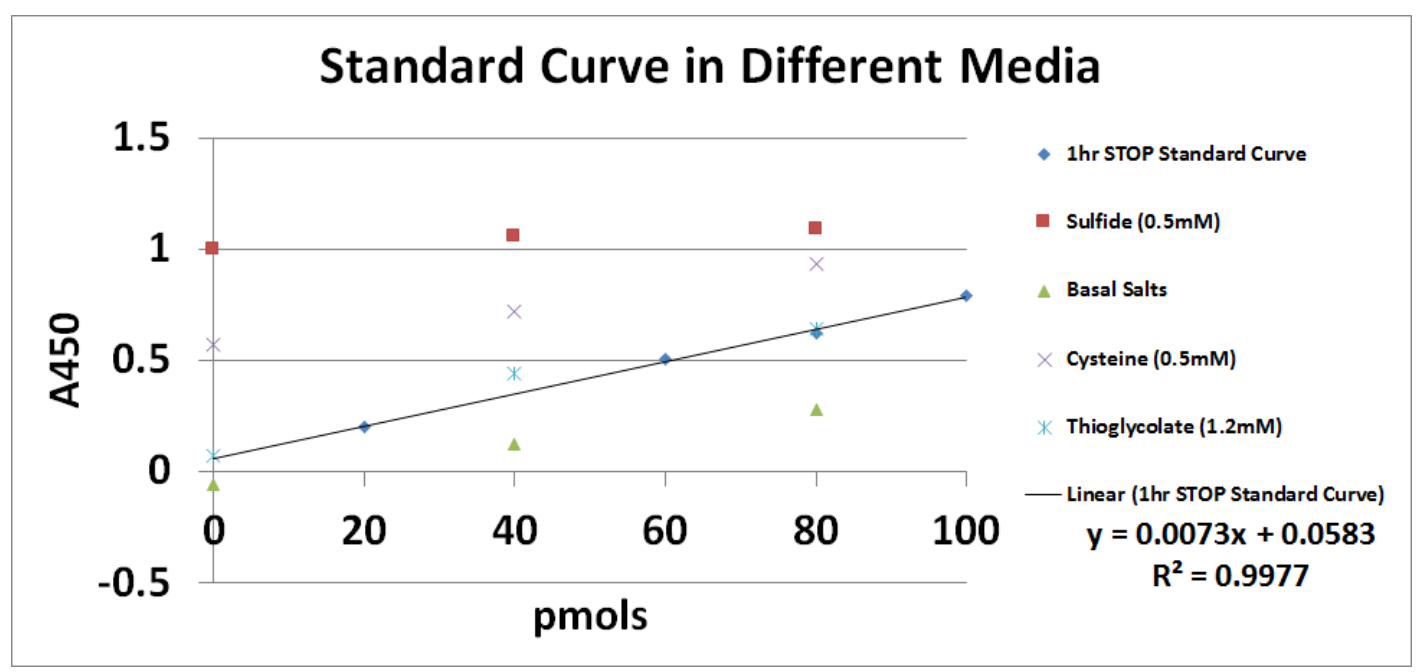

Figure 5-7: Media components disrupt pyridine nucleotide assay. Sulfide $(0.5 \mathrm{mM})$, cysteine $(0.5 \mathrm{mM})$, thioglycolate $(1.2 \mathrm{mM})$ and MO basal salts were added to standard curves mixtures and assayed for $\mathrm{A}_{450}$. The line represents the standard curve as determined by pure $\mathrm{NAD}^{+}$without additional amenities. 
Chapter 6: General discussion and concluding remarks 


\section{Section 6.1. Overview}

Bacteria have developed machinery to couple oxidative processes to energy conversion through the reduction of different terminal electron acceptors. Additionally, bacteria can undergo substrate-level phosphorylation when a terminal electron acceptor is limited. For SRM, sulfate reduction is coupled to the oxidation of many different substrates, including: $\mathrm{H}_{2}$, lactate, pyruvate, or formate. In many SRM, sulfate is not the only terminal electron acceptor that can be used. For example, the reduction of sulfite or thiosulfate is sufficient enough to support respiration (Muyzer et al. 2008). However, it is presumed that when thiosulfate is used for energy conversion, its reduction is coupled to the proton motive force as the reduction of thiosulfate is an endergonic reaction (Stoffels et al. 2012).

A strict anaerobe such as $\mathrm{DvH}$ can sustain growth in sulfate-rich and sulfate-limited environments because of its ability to use many different substrates for its respiration, explaining why $\mathrm{DvH}$ is nearly ubiquitous in nature. As $\mathrm{DvH}$ can maintain sustainable growth in many differing environments, a regulatory network that determines which substrates, and in what order, to use from the surrounding environment is critical (Barton et al. 2007). One regulator, Rex, has recently been identified to sense the rise in $\mathrm{NADH} / \mathrm{NAD}^{+}$ratio caused by limitations in the electron acceptor (i.e. sulfate, sulfite or thiosulfate) as a result of metabolism being slowed or halted (Brekasis et al. 2003). Rex binds to a promoter region upstream of genes responsible for energy conversion processes during respiration (i.e. sulfate reduction); however, Rex dissociates when NADH levels rise, a consequence of slowed metabolism, which consequently increases expression for those genes that code for sulfate reduction processes (McLaughlin et al. 2010).

The work presented here confirmed these traits for Rex $\mathrm{x}_{\mathrm{DvH}}$ on sat by in vitro assays (Figure 6-1). We also proposed that $\mathrm{Re}_{\mathrm{DvH}}$ controlled the electron flow internally towards 
sulfate reduction processes. The reasoning for this was due to a couple of conclusions determined in this study. First, Rex repressed genes that code for proteins that function in early sulfate reduction processes centered at the periplasmic membrane and within the cytoplasm (e.g. qmo $A B C D$ and $s a t)$. Secondly, as sulfate reduction rates are slowed (i.e. limited for a terminal electron acceptor) the $\mathrm{NADH} / \mathrm{NAD}^{+}$ratio was presumed to increase, which de-repressed Rex and lead to increased expression of these targets. Thridly, the Rex mutant is impaired for growth when thiosulfate is the sole electron acceptor. Thiosulfate is presumed to be reduced in the peirplasm (and not the cytoplasm) coupled to the proton motive force, which is different in location from where sulfate and sulfite are reduced.

\section{$\underline{\text { Respiration (Repression) }}$}

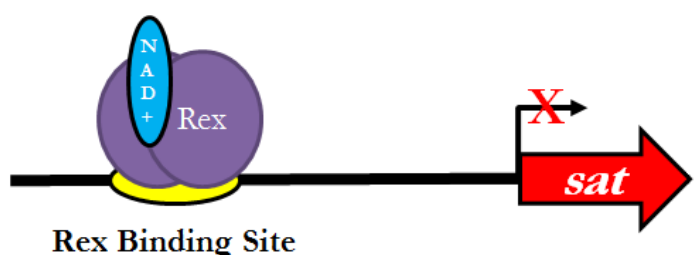

Fermentation (de-Repression)
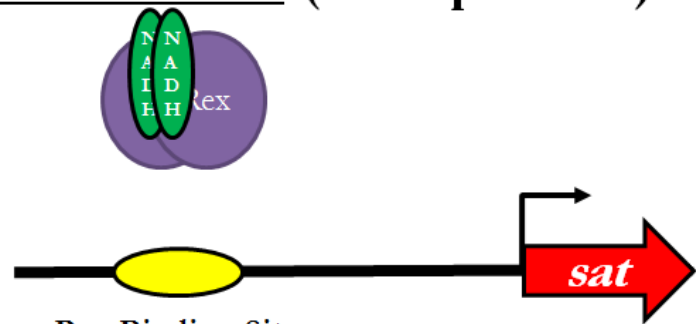

Rex Binding Site

Figure 6-1: Mechanism of $\operatorname{Rex}_{\mathrm{DvH}}$ in sulfate reduction. (LEFT) Respiration, Rex bound to $\mathrm{NAD}^{+}$, which allowed it to bind to the promoter region and repressed the downstream gene. (RIGHT) Fermentation, Rex bound to NADH, which caused a conformational change preventing Rex from binding to the promoter region, and therefore, allowed expression of the downstream gene. 
Additional terminal electron sensors previously studied in bacteria include ArcAB (Iuchi et al. 1988) and ResDE (Sun et al. 1996); however, these regulators are not found in DvH and seem to be specific to oxygen. The study presented here focused on a relatively new redox sensor, Rex, and specifically examined how Rex controlled the expression of several genes that code for proteins involved throughout the complete reduction of sulfate to sulfide. To that end, a Rex mutant and parenatal strain were grown by respiration or fermentation and analyzed for rates and extents of growth and transcript abundance of select sulfate reduction genes.

\section{Section 6. 2. Role of $\operatorname{Rex}_{\mathrm{DvH}}$}

The amino acid sequence for the predicted Rex regulator was modelled to several transcription factors, to NADH-dependent enzymes, and to Rex proteins. From these studies it was concluded that this putative protein was likely to be a Rex homolog, and not some other transcription factor. In this work, all the hallmark traits for Rex proteins were confirmed for the Rex homolog in DvH, $\operatorname{Rex}_{\mathrm{DvH}}$, including: protein-DNA interaction with the Rex-binding motif, de-repression by NADH and Rex targets are genes that code for proteins responsible for energy conversion processes.

Typically, Rex targets include many genes that code for proteins responsible for the oxidation of $\mathrm{NADH}$ to $\mathrm{NAD}^{+}$, allowing for the pyridine nucleotide pool to be reestablished and for respiration to proceed in a forward direction (Gyan et al. 2006). Targets repressed by Rex in other systems included: lactate dehydrogenase (ldh) and acetaldehyde dehydrogenase (adhE2) as observed in Clostridium acetobuylicum (Wietzke et al. 2012) and Staphylococcus aureus (Pagels et al. 2010); and NADH dehydrogenase (nuo or $n d b)$ as shown in Thermotoga maritima (Ravcheev et al. 2012), Streptomyces coelicolor A3(2) (Brekasis et al. 2003), and Bacillus subtilis (Gyan et al. 2006). In DvH, this would include NAD respiratory dehydrogenase (encoded by 
$n d h$ ), two separate pyridine dinucleotide-disulfide oxidoreductases (encoded by nox and DVU1974, respectively), and L-lactate dehydrogenase (encoded by ldh). However, upon examination of the targets predicted to be controlled by $\operatorname{Rex}_{\mathrm{DvH}}$, none of those mentioned above were included (Ravcheev et al. 2012). From a transcriptomic study (of a closely related SRB) that compared a Rex mutant to a parental strain grown by sulfate respiration with lactate, Rex did not appear to control any of the targets capable of oxidizing NADH mentioned above (Kuehl et al. 2014). In fact, the only protein known to be associated with coupling NADH oxidation to proton pumping that has been predicted to be controlled by $\operatorname{Rex}_{\mathrm{DvH}}$ is the $\mathrm{Rnf}$ complex, $d h c A-r n f C D G E A B-a p b E$ (Ravcheev et al. 2012). Interestingly, the expression for $m f C$ (the first gene in the functioning operon) appeared to be activated and not repressed by Rex in D. alaskensis G20 (Kuehl et al. 2014) and was confirmed in DvH by the work presented here. Therefore, the mechanism (or targets) by which Rex controls NADH levels in SRM may be different from the systems that have previously been characterized. Specifically, as we proposed here, Rex $x_{\mathrm{DvH}}$ appears to alter the electron flow between reductive processes within the cytoplasm and periplasm. The presence of Rex therefore maintains steady-state levels of sulfate reduction. Only when this process is slowed, as the case during pyruvate fermentation, did Rex increase expression of those genes that code for proteins responsible for the initial activation and reduction of sulfate to sulfite. The role of Rex in DvH may be to indirectly oxidize NADH by increasing the rate of sulfate reduction processes. This mechanism for Rex appears to be different from other systems that increase genes that directly function in oxidizing NADH (Brekasis et al. 2003; Sickmier et al. 2005).

Examination of gene fitness across the genome for Rex mutant and parental strain grown by sulfate respiration with lactate did not reveal many significant differences. Of the 
more than 3000 protein coding genes, only a few were shown to be altered in fitness greater than twofold between the two strains, and those genes considered to be essential in the Rex mutant were overlapping with those identified in the parental strain. This is quite different from previous studies that examined DvH under different stresses (e.g. nitrate (Korte et al. 2014)). Therefore, under these conditions (sulfate respiration with lactate) $\operatorname{Rex}_{\mathrm{DvH}}$ did not appear to be significant. These results were not a surprise as the deletion of rex in DvH was predicted to facilitate sulfate reduction.

Further examination of the Rex mutant by growth kinetic studies revealed that the deletion of rex impaired growth in thiosulfate-containing medium (with lactate or pyruvate as electron donor) compared with the parental strain. Reduction of thiosulfate was thought to occur by thiosulfate reductase, PhsAB (Aketagawa et al. 1985) within the periplasm. In this study we also proposed DVU3143-5 as a potential thiosulfate reductase in the periplasmic membrane that is linked to the proton motive force as was characterized for PhsABC in $S$. enterica (Stoffels et al. 2012). Rex $x_{\mathrm{DvH}}$ was not predicted to control either of these targets in $\mathrm{DvH}$, and transcript analysis performed in this study for $p h s A$ expression concluded this finding as well. Therefore, the role of either of these thiosulfate reductases was more likely to be controlled by growth conditions or $\operatorname{Rex}_{\mathrm{DvH}}$ indirectly. Further examination of DVU3143-5 is currently underway. From these findings, a model for thiosulfate reduction in DvH was proposed, which concluded that electrons were funneled through the menaquinone pool, possibly supplied by QrcABCD, and reduced thiosulfate while consuming the proton motive force at the periplasm. Additionally, the deletion of rex disrupted the flow of electrons to thiosulfate reductase, and instead forced the electrons to the cytoplasm for sulfate reduction, through overexpression of qmo $A B C$ and sat. Therefore, 
the deletion of rex would limit thiosulfate reduction and thus limit growth when thiosulfate was the sole terminal electron acceptor, as was observed in this study.

The transcript studies presented in this work, although not inclusive for all Rex $\mathrm{x}_{\mathrm{DvH}}$ targets, revealed that Rex ${ }_{\mathrm{DvH}}$ was most effective at modulating those genes that code for proteins responsible for early steps in the complete reduction of sulfate, although $\operatorname{Rex}_{\mathrm{DvH}}$ was predicted to control many genes. Rex $\operatorname{DvH}_{\mathrm{D}}$ repressed the expression of $s a t, a p s A, p p a C$, and adk; but not $d s r A$, nor $p h s A$. Interestingly, $d s r C$, not predicted to be controlled by Rex ${ }_{\text {DvH }}$, appeared to be activated by Rex $\mathrm{D}_{\mathrm{DvH}}$. The specifics to how Rex $\mathrm{x}_{\mathrm{DvH}}$ more strongly controls expression of some genes and not others is likely to be based on the position of $\operatorname{Rex}_{\mathrm{DvH}}$ binding site relative to the downstream start codon, additional binding-sites and thus regulators (and their signal molecule(s)) that act within the promoter, and the conservation (i.e. strength) of the Rex $x_{\mathrm{DvH}}$-binding site. However, initial examination of these sequences did not reveal any correlation between strongly and weakly controlled targets for Rex $\mathrm{D}_{\mathrm{DvH}}$. For example, sat and $d s r A$ (a strongly and weakly controlled target of $\operatorname{Rex}_{\mathrm{DvH}}$, respectively) have similar Rex ${ }_{\text {DvH- }}$ binding motifs and position relative to the downstream start codon, similar in vitro calculated dissociation constants, but significantly different transcript expression responses in a strain deleted for rex compared to a parental strain. Therefore, the complete mechanism for Rex $x_{\mathrm{DvH}}$ activity has only begun to be determined, with additional studies required for complete characterization for the role of $\operatorname{Rex}_{\mathrm{DvH}}$ and its role in the regulation of sulfate reduction. Overall, from these studies it was clear that Rex $x_{\text {vvH }}$ was important for facilitating sulfate reduction within the cytoplasm, either by increasing sulfate uptake into the cell or by increasing gene expression for those genes that code for proteins responsible for the early steps in sulfate reduction. 


\section{Section 6.3. Future plans...where to next?}

From this work it is clear that $\operatorname{Rex}_{\mathrm{DvH}}$ is involved in sulfate reduction, and specifically repressing genes (e.g. sat) that code for proteins responsible for early steps in the sulfate reduction pathway. It would also appear that $\operatorname{Rex}_{\mathrm{DvH}}$ acts as a molecular switch that alters the flow of electrons for reductive processes between the cytoplasm and periplasm (or periplasmic membrane). Rex $\mathrm{DvH}_{\mathrm{Du}}$ has been confirmed in vitro to act with predicted $\operatorname{Rex}_{\mathrm{DvH}^{-}}$ binding sites and the affinity of $\operatorname{Rex}_{\mathrm{DvH}}$ for these sites have been determined. Additional experiments could be performed to determine all targets of Rex $\mathrm{x}_{\mathrm{DvH}}$ within the genome and not just those examined in this study. For example, Chromatin Immunoprecipitation (ChIP) would accomplish this goal and has been done successfully for Rex in S. coelicolor (StrainDamerell 2010).

In regard to the Rex ${ }_{\mathrm{DvH}}-$ binding site, the specific contribution of each base within the consensus is not known. Several studies have been performed to begin to address this question for other organisms (Brekasis et al. 2003; Gyan et al. 2006), and was examined for $\mathrm{DvH}$ in this study. Therefore, a more thorough examination of each Rex $\mathrm{DvH}_{\mathrm{v}}$-binding site would be required to understand better how the sequence affects binding and, therefore, how $\operatorname{Rex}_{\mathrm{DvH}}$ regulates gene transcription. The necessary analyses would include adapting the protocols performed on sat in this study to each of the promoters regulated by $\operatorname{Rex}_{\mathrm{DvH}}$. Addition of single base deletion or substitution within the Rex $\mathrm{DvH}_{\mathrm{H}}$-binding site may also be informative.

In addition to the specific sequence, other factors play a role in gene regulation, including (Rojo 1999; Lee et al. 2012): 1) distance between translation start codon and regulator binding site, 2) additional regulators and therefore external signals (stress), 3) order of addition of the components required to activate or repress transcription, 4) DNA 
accessibility (i.e. supercoiling), 5) RNAP holoenzyme availability and subunits (i.e. $\sigma$-factor used) and 6) stability of the macromolecules (i.e. protein or mRNA).

To begin to understand some of these factors additional in vitro studies were undertaken. For example, to determine the TSS for sat, 5'-RACE was conducted on mRNA isolated from $\mathrm{DvH}$ grown by sulfate respiration with lactate and by fermentation with pyruvate. A more high-throughput analysis, e.g. 5'-RNA-sequencing, had been performed previously on DvH grown by sulfate respiration (Price et al. 2011). Fom the work presented in this study, the TSS was dependent on the medium that the cells were actively growing. Complementary studies, i.e. EMSA, transcript and protein expression studies, of genes that code for proteins involved in sulfate reduction processes not studied here would be insightful and would further our understanding of gene regulation. The Rex mutant did not affect the TSS, a result that was unexpected; therefore, a similar analysis on the Rex mutant would also be informative.

The in vitro DNA-binding assays performed here have determined that Rex $\mathrm{x}_{\mathrm{DvH}}$ likely plays a role in the regulation of over 50 genes. However, expression studies determining the in vivo role of $\operatorname{Rex}_{\mathrm{DvH}}$ has only been determined for a handful of these genes, including sat, $a p s A$, and $d s r A$. Therefore, a more exhaustive analysis of the remaining genes would further characterize the role of $\operatorname{Rex}_{\mathrm{DvH}}$ in sulfate respiration processes. A more high-throughput approach, including microarray of the Rex mutant in comparison to the parental strain, would facilitate this remaining question. A similar approach was performed on the closely related Desulfovibionales, D. alaskensis G20, and several targets not predicted to be controlled by Rex were identified, as well as confirming previous predictions (Kuehl et al. 2014). DvH and D. alaskensis G20 share $\sim 50 \%$ of their genome and a few of the targets for Rex are not shared between the strains (e.g. $p h s B A$, and $\cos M K L X U H A F$ ). Therefore, it would be 
reasonable to postulate that Rex may control targets in a similar manner (i.e.

repress/activate), but the overall response to the environment may be achieved differently and related to how each strain has adapted to their environmental niches.

Finally, the Rex mutant was shown to be impaired for growth when grown by thiosulfate respiration with lactate or pyruvate compared to its parental strain. This phenomenon has not been observed for Rex, although most studies to-date have not been on SRM, except in D. alaskensis G20 that only examined growth by sulfate respiration with lactate (Kuehl et al. 2014). In DvH, PhsAB (Findley et al. 1970) and DsrAB (Parey et al. 2010) have been shown to reduce thiosulfate, but in this work Rex $x_{\operatorname{DvH}}$ was shown to not control the expression of the encoding genes. Further examination and characterization of DVU3143-5 would further our understanding of the metabolism of thiosulfate reduction in DvH. Furthermore, additional work is needed to fully characterize the regulators that signal cellular nutrient and energy status that are integrated at the level of control of sulfate reduction. The work presented here was part of this effort to further characterize the process of sulfate reduction. 


\section{Chapter 7: $\quad$ Materials and methods}

This chapter includes the methods for the construction of the Rex marker-exchange deletion that was published by Frontiers in Microbiology, April 2014 I doi: 10.3389/fmicb.2014.00153. It is presented here with minor revisions.

This chapter includes the methods for the in vitro and in vivo characterization of Rex $\mathrm{Dv}_{\mathrm{Du}}$ that will be published by Journal of Bacteriology, January 2015 I doi: 10.1128/JB.02083-14. It is presented here with minor revisions.

Additionally, this chapter includes methods that are currently in prep, for submission to Applied and Environmental Microbiology.

RexDvH alters the electron flow between internal and external reduction processes in Desulfovibrio vulgaris Hildenborough.

G. A. Christensen ${ }^{1,2}$, S. R. Fels ${ }^{2,3}$, G.M. Zane ${ }^{1,2}$, Younkin A, D ${ }^{1,2}$, M.M.L. Sousa ${ }^{4,5}$, C.J. Petzold $^{2,6}$, A.P. Arkin ${ }^{2,6}$, J. D. Wall ${ }^{1,2}$

${ }^{1}$ Department of Biochemistry, University of Missouri, Columbia MO, USA

${ }^{2}$ Ecosystems and Networks Integrated with Genes and Molecular Assemblies, Berkeley CA, USA

${ }^{3}$ Department of Molecular Microbiology and Immunology, University of Missouri, Columbia MO, USA

${ }^{4}$ Department of Cancer Research and Molecular Medicine, Norwegian University of Science and Technology (NTNU), N-7489 Trondheim, Norway

${ }^{5}$ The Proteomics and Metabolomics Core Facility (PROMEC) at NTNU and the Central Norway Regional Health Authority, Trondheim, Norway

${ }^{6}$ Physical Biosciences Division, Lawrence Berkeley National Laboratory, Berkeley, CA, USA 


\section{Section 7.1. Protein sequence modelling}

The protein sequences for Rex for T. aquaticus, S. coelicolor, B. subtilis, and DvH were obtained from NCBI (Thompson et al. 1994) and protein identity calculated with BioEdit (Hall 1999). DVU0916 protein sequence was modelled by SWISS-MODEL (Arnold et al. 2006; Guex et al. 2009; Kiefer et al. 2009; Biasini et al. 2014). This database includes $>385,000$ chains encompassing 68493 unique sequences. The sequence was first automated via sequence alignment to the database for the "best-fit" model. This was followed by alignment to two solved Rex structures (pdb:3IKT and $1 \mathrm{XCB}$ ) as well as additional targets discussed elsewhere. For the alignment mode, DVU0916 protein sequence was first aligned to Rex from T. aquaticus with LAlign (Huang et al. 1991) (parameters: matrix file BL50 (15/-5), gapopen/ext: 14/-4 E(limit) 0.05)) resulting in a 45.8\% identity, 201Aa overlap, BLAST probability 5.5e-47. This template-target alignment was then used to model DVU0916 to pdb 3IKT chains A and B (2.26 ̊́ crystal structure of a Rex-family repressor/DNA/NAD ${ }^{+}$ complex from T. aquaticus, (McLaughlin et al. 2010)), and pdb 1XCB chains A, B, C, D, E, F

and G $(2.90 \AA ̊$ crystal structure of a Rex-family repressor/NADH complex from T. aquaticus (Sickmier et al. 2005)). Visual Molecular Dynamics (VMD) (Humphrey et al. 1996) and PV (protein viewer) were used to visualize the threaded structure. 


\section{Section 7. 2. Basic supplies and protocols}

\section{Section 7. 2. 1. Antibiotics and inhibitors}

Inhibitors for DvH strains were as follows: Geneticin ${ }^{\circledR}(\mathrm{G} 418,400 \mu \mathrm{g} / \mathrm{ml})$, spectinomycin (Sp, $100 \mu \mathrm{g} / \mathrm{ml})$, kanamycin (Km, $50 \mu \mathrm{g} / \mathrm{ml}$ ), ampicillin (Ap, $100 \mu \mathrm{g} / \mathrm{ml}$ ), 5-fluorouracil (5FU, $40 \mu \mathrm{g} / \mathrm{ml}$ ) or molybdate (Mo, $1 \mathrm{M}$ stock) obtained from Thermo Fisher Scientific (Waltham, MA), Sigma-Aldrich (St. Louis, MO), or Gold Biotechnologies ${ }^{\circledR}$ (St. Louis, MO).

\section{Section 7. 2. 2. Primers/Oligonucleotides}

Oligonucleotides necessary for these studies were purchased from Integrated DNA Technologies (IDT, Coralville, IA). The lyophilized pellet was rehydrated to a final stock concentration of $100 \mu \mathrm{M}$ and stored at $-20^{\circ} \mathrm{C}$ until use. Primers are kept at $4^{\circ} \mathrm{C}$ if they are to be used frequently within the week. Primer concentrations and quality (ratio $A_{260} / A_{280}$ ) were calculated from Nanodrop ND-1000 spectrophotometric readings (Thermo Fisher Scientific) after initial rehydration. Primers necessary for a given project are listed in separate tables in their specified section.

\section{Section 7.2.3. Sequence analysis}

Sequence analysis was performed by University of Missouri DNA Core Facility. For each DNA sample to be sequence, a $16 \mu \mathrm{l}$ reaction volume was provided. This included $\sim 1000 \mathrm{ng}$ DNA template (50-500 ng PCR or plasmid DNA), 20 pmol specified primer. For templates that were GC-rich or failed the first read, 5\% (vol/vol) DMSO was added per their suggestion. 


\section{Section 7. 2. 4. Culturing techniques for DvH}

The growth of all cultures was measured in Balch tubes by optical density (OD600 nm) with a Spectronic ${ }^{\text {TM }}$ GENESYS ${ }^{\text {TM }} 20$ spectrophotometer (Thermo Fisher Scientific). E. coli strains were grown at $37^{\circ} \mathrm{C}$ aerobically in $5 \mathrm{ml} \mathrm{LC}$ medium containing per liter $(10 \mathrm{~g}$ tryptone, $5 \mathrm{~g}$ sodium chloride, and $5 \mathrm{~g}$ yeast extract), $\mathrm{pH}$ 7.0. DvH strains were started from freezer stocks that contained $10 \%(\mathrm{vol} / \mathrm{vol})$ glycerol in growth medium and frozen at $-80^{\circ} \mathrm{C}$. Growth medium was generally MO medium (Zane et al. 2010) supplemented with $0.1 \%$ (wt/vol) yeast extract and $60 \mathrm{mM}$ lactate with $30 \mathrm{mM}$ sulfate (MOYLS4). The medium was reduced with $1.2 \mathrm{mM}$ sodium thioglycolate and $\mathrm{pH}$ was adjusted to $\mathrm{pH} 7.2$ with $12 \mathrm{M} \mathrm{HCl}$. DvH cultures were grown in an anaerobic chamber (Coy Laboratory Product, Inc., Grass Lake, MI) overnight until stationary phase (OD600 >1). The atmosphere of the chamber was approximately $95 \% \mathrm{~N}_{2}$ and $5 \% \mathrm{H}_{2}$. A $2 \%$ (vol/vol) inoculum was used to start $5 \mathrm{ml}$ cultures in defined medium $(\mathrm{MO})$ containing lactate $(60 \mathrm{mM}, \mathrm{L})$ or pyruvate $(60 \mathrm{mM}, \mathrm{P})$ as the electron donor and sulfate $(30 \mathrm{mM}, \mathrm{S} 4)$, sulfite $(40 \mathrm{mM}, \mathrm{S} 3)$ or thiosulfate $(30 \mathrm{mM}, \mathrm{T} 3)$ as the electron acceptor or MOYPyr (60 mM pyruvate, supplemented with $0.1 \%$ (wt/vol) yeast extract and $0.5 \mathrm{mM}$ cysteine). For pyruvate-fermenting cultures, cysteine was provided as a sulfur source and reductant. DvH did not grow on yeast extract alone in the absence of pyruvate. Validation of this is shown in Chapter 5.

\section{Section 7. 2. 5. Electroporation}

Standard electroporation protocols were followed with an ECM 630 electroporator (BTX) (Keller et al. 2011), with parameters: $1500 \mathrm{~V} 250 \Omega$ and 25uF. Typically, 300-500 ng of template DNA was electroporated into $50 \mu \mathrm{l}$ of a $50 \mathrm{ml}$ culture that had been washed with TE buffer and resuspended into 300-500 $\mu \mathrm{l}$ (depending on density of the culture and the 
number of samples to be electroporated). A 1-ml aliquot of rich medium (MOYLS4) is added to the samples and let recover overnight at $34^{\circ} \mathrm{C}$ within an anaerobic chamber. The next day the cells are plated (typically $10 \mu \mathrm{l}, 100 \mu \mathrm{l}$ and $880 \mu \mathrm{l}$ ) on the desired media.

\section{Section 7. 2.6. DNA purification}

Genomic DNA (Wizard® Genomic DNA Purification Kit, Promega, Madison WI) and plasmid (GeneJET plasmid kit, Thermo Fisher Scientific) were purified according to manufacturer's protocol from a 1.5-ml aliquot of an overnight culture (OD600>1). DNA fragments (via PCR or primer annealing) were purified according to manufacturer's protocol (Wizard® SV Gel and PCR Clean-Up System [Promega]). DNA concentrations and quality (ratio A260/A280) were calculated from Nanodrop ND-1000 spectrophotometric readings (Thermo Fisher Scientific).

\section{Section 7. 2. 7. RNA purification}

Samples to be analyzed for RNA content were cultured as described in the culturing DvH section. At the specified optical density, 4-ml samples were spun down anaerobically at $34{ }^{\circ} \mathrm{C}$ (10 min at $5600 \times \mathrm{g}$ ) and resuspended in $1 \mathrm{ml}$ TRI Reagent ${ }^{\circledR}$ (Sigma-Aldrich). RNA was purified based on phenol extraction and ethanol precipitation methods previously described (Chomczynski et al. 1987; 2006). RNA quality was assessed visually in an agarose denaturing gel and an A260/A280 ratio >1.8 was required (Figure 7-1). RNA samples were treated with TURBO $^{\text {TM }}$ DNase (Life Technologies, Carlsbad CA) according to the manufacturer's protocol and confirmed by PCR to be free of gDNA (Figure 7-1). 
A

B

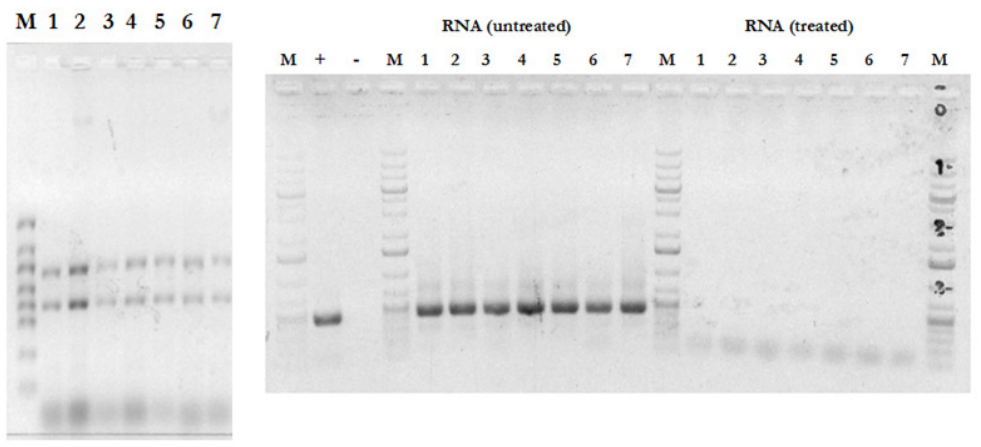

Figure 7-1: RNA quality assurance. A) RNA gel. Seven RNA samples after ethanol precipitation are electrophoresed on a $1.5 \%$ (wt/vol) denaturing agarose gel. The marker is RiboRuler High Range (Thermo Fisher Scientific). B) DNA contamination screen of seven samples after RNA isolation protocol. M) $1 \mathrm{~kb}$ plus ladder (Fermentas), +/- with or without gDNA DvH (+/controls). RNA (untreated): RNA samples after initial RNA purification. RNA (treated): Isolated RNA samples after DNase Treatment. Samples properly treated no longer generate a band ( $500 \mathrm{bp})$.

\section{Section 7. 2. 8. Polymerase Chain Reaction (PCR)}

All DNA to be amplified by PCR followed standard protocols. For typical reactions (e.g. for screening purposes) either Taq DNA polymerase (NEB) or Bullseye Taq DNA polymerase (MIDSCI, St Louis, MO) were used. When accuracy was essential (e.g. cloning) Herculase ${ }^{\circledR}$ II Fusion DNA polymerase (Agilent, Santa Clara, CA) was used. Reaction mixes were set up according to the manufacturer's protocol, with the annealing temperature set for $1-5^{\circ} \mathrm{C}$ less than the $T_{m}$ of the primers. If multiple bands or no product was obtained after 30 cycles, the experiment was set-up a second time with 5\% (vol/vol) DMSO added. 


\section{Section 7.3. Strains and plasmids}

All strains in this study are listed in Table 7-1 and plasmids in Table 7-2. Strains designated with JW33XX were those I personally constructed. Additional strains were constructed by the Wall laboratory, specifically Grant Zane, or were purchased as annotated. Plasmids designated with pMO33X were those I personally constructed. Primers necessary for strain generation are listed in Table 7-3. Plasmids are confirmed by Sanger sequencing by DNA Core Facility at University of Missouri.

The general strategy used to generate a desired deletion strain is described in great detail elsewhere (Keller et al. 2009; Keller et al. 2011; Keller et al. 2011). In brief, a plasmid is constructed by the process of Sequence Ligation Independent Cloning (SLIC)(Li et al. 2007), which contained two flanking homologous regions to the sequence to be deleted.

Additionally, this plasmid has a selectable and counter-selectable marker for isolation purposes. This sequence-confirmed plasmid is transformed into the designated DvH strain and selected on the appropriate antibiotics followed by confirming the strain by Southern blot (Zane et al. 2010). 
Table 7-1: Strains

\begin{tabular}{|c|c|c|}
\hline Strain & Genotype or relevant characteristics ${ }^{a}$ & $\begin{array}{l}\text { Source or } \\
\text { reference }\end{array}$ \\
\hline \multicolumn{3}{|l|}{ Strain: } \\
\hline \multicolumn{3}{|l|}{ E. coli } \\
\hline $\begin{array}{r}\alpha \text {-Select (Silver } \\
\text { Efficiency) }\end{array}$ & $\begin{array}{l}\text { deoR endA1 recA1 relA1 gyrA96 hsdR17 }\left(\mathrm{r}_{\mathrm{k}}{ }^{-}, \mathrm{m}_{\mathrm{k}}{ }^{+}\right) \text {supE44 thi-1 } \Delta(\text { lacZYA- } \\
\text { argFV169) } 980 \mathrm{dl} \text { acZ } \Delta \mathrm{M}^{-} 5 \mathrm{~F}^{-}\end{array}$ & Bioline \\
\hline $\begin{array}{l}\text { BL21 (DE3) } \\
\text { competent cells }\end{array}$ & E. coli $\mathrm{B} \mathrm{F}^{-} d c m$ ompT $h s d S\left(\mathrm{r}_{\mathrm{B}^{-}} \mathrm{m}_{\mathrm{B}^{-}}\right) \operatorname{gal} \lambda(\mathrm{DE} 3)$ & Agilent \\
\hline \multicolumn{3}{|c|}{ Desulfovibrio vulgaris Hildenborough } \\
\hline ATCC29579 & Wild-type D. vulgaris Hildenborough; 5-FUs & ATCC \\
\hline JW710 & $\begin{array}{l}\text { WT } \Delta u p p ; 5-\mathrm{FU} \text { ( used as "WT" control for DvH growth kinetics in this } \\
\text { study; parent strain for deletions; retains pDV1 present in WT) }\end{array}$ & $\begin{array}{l}\text { (Keller et al. } \\
\text { 2009) }\end{array}$ \\
\hline JW3311 & JW710 $\Delta$ DVU0916::(npt upp); Kmr; 5-FUs ( $\Delta$ rex marker exchange) & $\begin{array}{l}\text { (Korte et al. } \\
\text { 2014) }\end{array}$ \\
\hline JW3317 & $\begin{array}{l}\text { JW710 } \Delta \text { DVU0916-0915::(npt upp); Km } ; 5-\mathrm{FU}^{\mathrm{s}}(\Delta \text { rex and } \Delta D V U 0915 \text { marker } \\
\text { exchange) }\end{array}$ & This study \\
\hline JW3319 & JW710 $\Delta$ DVU0916; Kms; 5-FUr ( $\Delta$ rex marker-less deletion) & This study \\
\hline JW9011 & JW710 $\Delta \mathrm{DVU} 2547::(n p t$ upp); Kmr; 5-FUs ( $\Delta h c p \mathrm{R}$ marker exchange) & $\begin{array}{l}\text { (Zhou et al. } \\
\text { 2012) }\end{array}$ \\
\hline JW9293 & 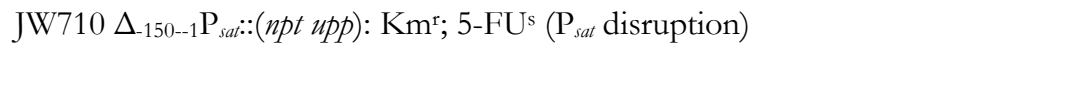 & $\begin{array}{l}\text { (Christensen } \\
\text { et al. 2014) }\end{array}$ \\
\hline JW9312 & JW710; Kms, 5-FUr (sat promoter restored) & $\begin{array}{l}\text { (Christensen } \\
\text { et al. 2014) }\end{array}$ \\
\hline JW9314 & JW9293 G-147A Psat; Kms; 5-FUr (G -147 A) & $\begin{array}{l}\text { (Christensen } \\
\text { et al. 2014) }\end{array}$ \\
\hline JW9316 & JW9293 GTA-147--145ACG $\mathrm{P}_{\text {sat }} ; \mathrm{Km}^{\mathrm{s}} ;$ 5-FUr (IR1) & $\begin{array}{l}\text { (Christensen } \\
\text { et al. 2014) }\end{array}$ \\
\hline JW9318 & JW9293 CAC-136-134TGT Psat; Kms; 5-FU (IR2) & $\begin{array}{l}\text { (Christensen } \\
\text { et al. 2014) }\end{array}$ \\
\hline JW9320 & 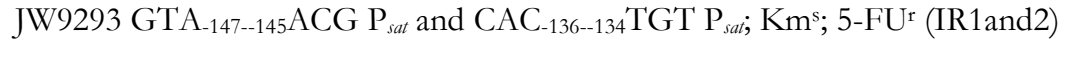 & $\begin{array}{l}\text { (Christensen } \\
\text { et al. 2014) }\end{array}$ \\
\hline JW9011 & JW710 $\Delta \mathrm{DVU} 2547::(n p t$ upp); Kmr; 5-FUs ( $\Delta h c p \mathrm{R}$ marker exchange) & $\begin{array}{l}\text { (Zhou et al. } \\
\text { 2012) }\end{array}$ \\
\hline GZ0481 & $\begin{array}{l}\text { Genome position } 2680507:: \operatorname{Tn} 5-\mathrm{RL} 27 \text {; insertion } 273 \text { bp from predicted AUG } \\
\text { start codon within DVU2567; } \mathrm{Km}^{\mathrm{r}} \text { (LysX mutant) }\end{array}$ & $\begin{array}{c}\text { Wall } \\
\text { Laboratory }\end{array}$ \\
\hline GZ1801 & $\begin{array}{l}\text { Genome position } 2804495:: T n 5-R L 27 \text {; insertion } 178 \text { bp from predicted AUG } \\
\text { start codon within DVU0269; Km (DVU2690 mutant) }\end{array}$ & $\begin{array}{c}\text { Wall } \\
\text { Laboratory }\end{array}$ \\
\hline GZ4443 & $\begin{array}{l}\text { Genome position 1238583::Tn5-RL27; insertion } 52 \text { bp from predicted AUG } \\
\text { start codon within DVU1142; } \mathrm{Km}^{\mathrm{r}} \text { (DVU1142 mutant) }\end{array}$ & $\begin{array}{l}\text { Wall } \\
\text { Laboratory }\end{array}$ \\
\hline GZ5190 & $\begin{array}{l}\text { Genome position 2364109::Tn5-RL27; insertion } 118 \text { bp from predicted AUG } \\
\text { start codon within DVU5190; } \mathrm{Km}^{\mathrm{r}} \text { (RocR mutant) }\end{array}$ & $\begin{array}{l}\text { Wall } \\
\text { Laboratory }\end{array}$ \\
\hline GZ7888 & $\begin{array}{l}\text { Genome position 64424::Tn5-RL27; insertion } 535 \text { bp from predicted AUG } \\
\text { start codon within DVU0057; } \mathrm{Km}^{\mathrm{r}} \text { (DVU0057 mutant) }\end{array}$ & $\begin{array}{l}\text { Wall } \\
\text { Laboratory }\end{array}$ \\
\hline GZ8929 & $\begin{array}{l}\text { Genome position 218225::Tn5-RL27; insertion } 366 \text { bp from predicted AUG } \\
\text { start codon within phsA: } \mathrm{Kmr} \text { (phsA mutant) }\end{array}$ & $\begin{array}{l}\text { Wall } \\
\text { Laboratory }\end{array}$ \\
\hline GZ12386 & $\begin{array}{l}\text { Genome position 2900951::Tn5-RL27; insertion } 102 \text { bp from predicted AUG } \\
\text { start codon within DVU2799; } \mathrm{Km}^{\mathrm{r}} \text { (DVU2799 mutant) }\end{array}$ & $\begin{array}{l}\text { Wall } \\
\text { Laboratory }\end{array}$ \\
\hline GZ12635 & $\begin{array}{l}\text { Genome position 832548::Tn5-RL27; insertion } 80 \text { bp from predicted AUG } \\
\text { start codon within DVU0744; Km (DVU0744 mutant) }\end{array}$ & $\begin{array}{l}\text { Wall } \\
\text { Laboratory }\end{array}$ \\
\hline GZ12985 & $\begin{array}{l}\text { Genome position 2905014::Tn5-RL27; insertion } 75 \text { bp from predicted AUG } \\
\text { start codon within DVU2802; } \mathrm{Km}^{\mathrm{r}} \text { (DVU2802 mutant) }\end{array}$ & $\begin{array}{c}\text { Wall } \\
\text { Laboratory }\end{array}$ \\
\hline
\end{tabular}

"Km, kanamycin; Sp, spectinomycin; Ap, ampicillin; 5-FU, 5-fluorouracil; superscript " $r$ " or "s", resistance or sensitivity 
Table 7-2: Plasmids

\begin{tabular}{|c|c|c|}
\hline Plasmid & Genotype or relevant characteristics ${ }^{a}$ & $\begin{array}{l}\text { Source or } \\
\text { reference }\end{array}$ \\
\hline pET14b & 6XHis-tag fusion protein vector with $\mathrm{T} 7$ promoter & Novagen \\
\hline pMO719 & $\begin{array}{l}\mathrm{pCR} 8 / \mathrm{GW} / \mathrm{TOPO} \text { containing SRB replicon (pBG1); } \mathrm{Sp} \mathrm{p}^{\mathrm{r}} \text {; source of } \\
\mathrm{Sp}^{\mathrm{r}} \text { and } \mathrm{pUC} \text { ori fragment; for marker exchange suicide plasmid } \\
\text { construction }\end{array}$ & $\begin{array}{l}\text { (Keller et al. } \\
\text { 2009) }\end{array}$ \\
\hline pMO746 & $\begin{array}{l}\text { Source of } u p p \text { in artificial operon with } n p t \text { and } A p^{\mathrm{r}}-\mathrm{pUC} \text { ori; } \mathrm{P}_{n p t}-n p t- \\
u p p ; \mathrm{Km}^{\mathrm{r}} ; 5-\mathrm{FU} \text {; for marker exchange suicide plasmid } \\
\text { construction }\end{array}$ & $\begin{array}{l}\text { (Parks et al. } \\
\text { 2013) }\end{array}$ \\
\hline pMO3311 & $\begin{array}{l}\mathrm{Sp}^{\mathrm{r}} \text { and pUC ori from pMO719 plus } 1630 \text { bp upstream and } 1590 \mathrm{bp} \\
\text { downstream DNA regions from DVU0916 (rex) flanking the } \\
\text { artificial operon of } \mathrm{P}_{n p t}-n p t-u p p \text { from pMO746; marker exchange } \\
\text { deletion of rex } \mathrm{Sp}^{\mathrm{r}} ; \mathrm{Km}^{\mathrm{r}}\end{array}$ & $\begin{array}{l}\text { (Korte et al. } \\
\text { 2014) }\end{array}$ \\
\hline pMO3312 & $\begin{array}{l}\text { pET14b plus rex (without start codon, } 642 \text { bp); Ap }{ }^{\mathrm{r}} \text {; for Rex } \\
\text { expression in BL21 (DE3) competent cells. }\end{array}$ & $\begin{array}{l}\text { (Christensen et } \\
\text { al. 2014) }\end{array}$ \\
\hline pMO3313 & pMO9075 with DVU0916 (rex) constitutively expressed from $\mathrm{P}_{n p t}$ & $\begin{array}{l}\text { (Korte et al. } \\
\text { 2014) }\end{array}$ \\
\hline pMO3316 & $\begin{array}{l}\text { pMO9075 with DVU0916 (rex) and DVU0915 constitutively } \\
\text { expressed from } \mathrm{P}_{n p t}\end{array}$ & This study \\
\hline pMO3317 & $\begin{array}{l}\mathrm{Sp}^{\mathrm{r}} \text { and pUC ori from pMO719 plus } 1630 \mathrm{bp} \text { upstream of rex and } \\
669 \mathrm{bp} \text { downstream of DVU0915 DNA regions flanking the } \\
\text { artificial operon of } \mathrm{P}_{n p t}-n p t-u p p \text { from pMO746;marker exchange } \\
\text { deletion of rex and DVU0915 } \mathrm{Sp}^{\mathrm{r}} ; \mathrm{Km}^{\mathrm{r}}\end{array}$ & This study \\
\hline pMO3319 & $\begin{array}{l}\mathrm{Sp}^{\mathrm{r}} \text { and } \mathrm{pUC} \text { ori from pMO719 plus } 1630 \mathrm{bp} \text { upstream and } 1590 \mathrm{bp} \\
\text { downstream DNA region from DVU0916 (rex); marker-less } \\
\text { deletion plasmid; Sp }\end{array}$ & This study \\
\hline pMO9075 & $\begin{array}{l}\text { pMO719 containing } \mathrm{P}_{n p t} \text { for constitutive expression of } \\
\text { complementation constructs; pBG1 stable SRB replicon; } \mathrm{Sp}^{\mathrm{r}}\end{array}$ & $\begin{array}{l}\text { (Keller et al. } \\
\text { 2011) }\end{array}$ \\
\hline pMO9292 & $\begin{array}{l}\mathrm{Sp}^{\mathrm{r}} \text { and } \mathrm{pUC} \text { ori from pMO719 plus } 383 \mathrm{bp} \text { upstream and } 319 \mathrm{bps} \\
\text { downstream DNA regions from } \mathrm{P}_{\text {sat }}(-150--1) \text { flanking the artificial } \\
\text { operon of } \mathrm{P}_{n p t}-n p t-u p p \text { from pMO746; for marker exchange } \\
\text { mutagenesis; } \mathrm{Sp}^{\mathrm{r}} \text { and } \mathrm{Km}^{\mathrm{r}}\end{array}$ & $\begin{array}{l}\text { (Christensen et } \\
\text { al. 2014) }\end{array}$ \\
\hline pMO9311 & $\begin{array}{l}\mathrm{Sp}^{\mathrm{r}} \text { and } \mathrm{pUC} \text { ori from pMO719 plus } 403 \text { bp upstream and } 467 \mathrm{bp} \\
\text { downstream DNA regions from } \mathrm{P}_{\text {sat }}(-150) \text {; wild-type sequence; } \\
\mathrm{Sp}^{\mathrm{r}} \text {; for site directed mutagenesis }\end{array}$ & $\begin{array}{l}\text { (Christensen et } \\
\text { al. 2014) }\end{array}$ \\
\hline pMO9313 & $\begin{array}{l}\mathrm{Sp}^{\mathrm{r}} \text { and } \mathrm{pUC} \text { ori from pMO719 plus } 403 \text { bp upstream and } 467 \mathrm{bp} \\
\text { downstream DNA regions from } \mathrm{P}_{\text {sat }}(-150) ; \mathrm{G}_{-147} \mathrm{~A} \text { Psat; Sp } \mathrm{p}^{\mathrm{r}} \text {; site } \\
\text { directed mutagenesis }\end{array}$ & $\begin{array}{l}\text { (Christensen et } \\
\text { al. 2014) }\end{array}$ \\
\hline pMO9315 & $\begin{array}{l}\mathrm{Sp}^{\mathrm{r}} \text { and } \mathrm{pUC} \text { ori from pMO719 plus } 403 \mathrm{bp} \text { upstream and } 467 \mathrm{bp} \\
\text { downstream DNA regions from } \mathrm{P}_{\text {sat }}(-150) \text {; GTA-147--145 } \mathrm{ACG} \mathrm{P}_{\text {sat; }} \\
\mathrm{Sp}^{\mathrm{r}} \text {; for site directed mutagenesis }\end{array}$ & $\begin{array}{l}\text { (Christensen et } \\
\text { al. 2014) }\end{array}$ \\
\hline pMO9317 & $\begin{array}{l}\mathrm{Sp}^{\mathrm{r}} \text { and } \mathrm{pUC} \text { ori from pMO719 plus } 403 \mathrm{bp} \text { upstream and } 467 \mathrm{bp} \\
\text { downstream DNA regions from } \mathrm{P}_{\text {sat }}(-150) \text {; CAC-136-134TGT } \mathrm{P}_{\text {sat; }} \text {; } \\
\mathrm{Sp}^{\mathrm{r}} \text {; for site directed mutagenesis }\end{array}$ & $\begin{array}{l}\text { (Christensen et } \\
\text { al. 2014) }\end{array}$ \\
\hline pMO9319 & $\begin{array}{l}\mathrm{Sp}^{\mathrm{r}} \text { and } \mathrm{pUC} \text { ori from pMO719 plus } 403 \text { bp upstream and } 467 \mathrm{bp} \\
\text { downstream DNA regions from } \mathrm{P}_{\text {sat }}(-150) \text {; GTA-147--145 ACG } \mathrm{P}_{\text {sat }} \\
\text { and CAC-136-134TGT } \mathrm{P}_{\text {sat }} ; \mathrm{Sp}^{\mathrm{r}} \text {; for site directed mutagenesis }\end{array}$ & $\begin{array}{l}\text { (Christensen et } \\
\text { al. 2014) }\end{array}$ \\
\hline pRL27 & $\begin{array}{l}\left.\text { Tn5-RL27 ( } \mathrm{Km}^{\mathrm{r}} \text {-or R6 } \mathrm{K}\right) \text { delivery vector; for transposon } \\
\text { mutagenesis of DvH strains }\end{array}$ & $\begin{array}{l}\text { (Larsen et al. } \\
\text { 2002) }\end{array}$ \\
\hline
\end{tabular}




\section{Section 7. 3.1. Marker-exchange deletion of $r e x$}

A marker-exchange deletion (MED) of rex (JW3311) was constructed. In brief, a plasmid (designated pMO3311) was constructed by the process of SLIC (Li et al. 2007; Keller et al. 2009; Korte et al. 2014). pMO3311 included 1630 bases upstream of the putative AUG start codon and 1590 bases downstream of rex from DvH amplified from gDNA; 1885 bases from pMO719 that included the spectinomycin resistance marker and $\mathrm{pUC}$ ori amplified from pMO719; and a kanamycin resistance cassette coupled with a counter-selectable marker (designated as KanRupp) amplified from pMO746. This plasmid was electroporated into the $\triangle$ upp strain, JW710, with an ECM 630 electroporator (BTX) following common protocols with parameters: $1500 \mathrm{~V}, 250 \Omega$ and $25 \mathrm{uF}$ (Keller et al. 2011). The cells were recovered in 1 $\mathrm{ml}$ MOYLS4 (supplemented with $1 \mathrm{mM}$ sulfite) overnight at $34^{\circ} \mathrm{C}$ in a $1.5 \mathrm{ml}$ microfuge tube. Recovered cells were plated on MOYLS4 + G418 and incubated for 5 days. Individual colony forming units (cfus) were replica screened on MOYLS4, MOYLS4+sp, and MOYLS4+G418. Three cfus with the correct phenotype $\left(\mathrm{G} 418^{\mathrm{r}}, \mathrm{Sp}^{\mathrm{s}}\right)$ were toothpicked into $5 \mathrm{ml}$ cultures (MOYLS4+G418) and incubated overnight at $34^{\circ} \mathrm{C} . \mathrm{gDNA}$ was isolated from each culture and a southern blot (Brown 1993; Zane et al. 2010) was performed with restriction enzyme BglI (NEB). BgII-digested genomic DNAs were probed with a PCR fragment upstream of the rex gene. A DNA band of 4129 bps showed hybridization in the wild-type sample, compared with a fragment of 2645 bps for a correctly constructed MED in JW3311 (Figure 7-2). Rex mutant was further confirmed by sensitivity to molybdate (Figure 5-3). A correctly identified construct, MED strain for rex, was designated JW3311. Freezer stocks were made and contain 10\% (vol/vol) glycerol. 
A $\quad$ B

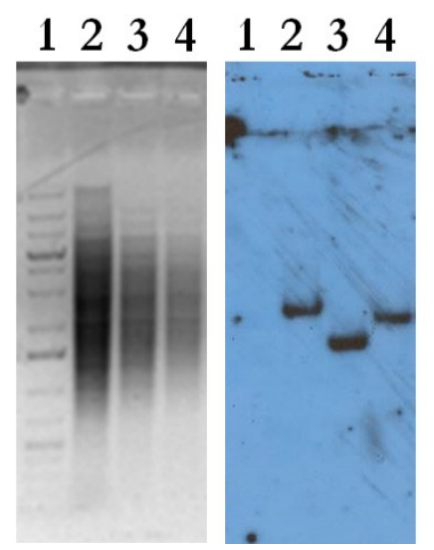

Figure 7-2: Rex mutants confirmed by Southern blot. A) DNA agarose gel of BgII-digested gDNA from parental (JW710), rex marker-exchange deletion (MED, JW3311) and rex marker-less deletion (MLD, JW3319) strains. Lanes: 1: ladder; 2, JW710; 3 rex MED; and 4, rex MLD. B) X-ray film of gDNA digested with BgII, and probed for an $\sim 1 \mathrm{~kb}$ fragment. Expected sizes are: JW710 4129 bp, JW3311 2488 bp and JW3319 3484 bp.

\section{Section 7. 3. 2. Marker-less deletion of rex}

A marker-less in frame deletion of rex (JW3319) was constructed. In brief, a plasmid (designated pMO3319) was constructed by the process of sequence and ligation independent cloning (Li et al. 2007; Keller et al. 2009; Korte et al. 2014). pMO3319 included 1630 bases upstream of the putative AUG start codon and 1590 bases downstream of rex from $\mathrm{DvH}$, and 1885 bases from pMO719 that included the spectinomycin resistance marker and pUC ori. This plasmid was electroporated into the rex marker exchange strain, JW3311 (Korte et al. 2014). The cells were recovered overnight in $1 \mathrm{ml}$ MOYLS4 and plated for single colonies in molten MOYLS4 +5 -FU for growth at $34^{\circ} \mathrm{C}$. After five days, colony forming units (cfus) were counted and screened by replica plating for $5-\mathrm{FU}^{\mathrm{r}}, \mathrm{Km}^{\mathrm{s}}$ and $\mathrm{Sp}^{\mathrm{s}}$ phenotypes. Five cfus 
from the MOYLS4 plate with the correct phenotype were toothpicked into $5 \mathrm{ml}$ MOYLS4 and incubated overnight at $34^{\circ} \mathrm{C}$. gDNA was purified and the deletion was confirmed by Southern blot (Figure 7-2) (Zane et al. 2010) and freezer stocks to 10\% (vol/vol) glycerol were made.

One final mutant, JW3317, was also constructed by the same protocol. This strain was a marker-less deletion of rex through the downstream gene, DVU0915. To generate this strain, a plasmid, pMO3317, was constructed to include the same upstream region as described above but included 669 bps of a homologous region downstream of DVU0915. This plasmid was then transformed into the marker-exchange strain, JW3311, and the normal strain construction protocol was followed.

\section{Section 7.3.3. Complementation strategy}

The strategy for complementation was to transform a plasmid containing the gene of interest back into the specified gene deleted strain (Zane et al. 2010). The gene of interest would be downstream of a constitutive promoter and, therefore, would be expressed most likely at a higher than normal level. Each plasmid was constructed by the process of SLIC (Li et al. 2007), generating the backbone from pMO9075 with primers pMO9075-SLIC-F and pMO9075-SLIC-R3. The sequence confirmed plasmid was electroporated into the desired DvH strain according to standard protocol and selected for spectinomycin resistance. Plasmid was then purified from the DvH strain and passed back through E.coli for amplification purposes and the sequence was confirmed.

\section{Section 7. 3. 4. Sat promoter mutants}

The protocol used to generate the strain lacking the sat promoter $\left(\Delta \mathrm{P}_{\text {sat }}\right)$, JW9293 conferring $\mathrm{Km}^{\mathrm{r}}$ and 5-FU', has previously been described (Korte et al. 2014). Briefly, 150 bp upstream 
of sat (-150 to -1$)$ was replaced with a cassette conferring resistance to kanamycin and a counter-selectable marker (upp, the gene encoding uracil phosphoribosyltransferase that confers 5-fluorouracil sensitivity to the 5-FU ${ }^{\mathrm{r}}$ parental strain). To accomplish the cassette insertion, a delivery plasmid (pMO9292) was constructed that contained the selectable cassette flanked by chromosomal regions from either side of the promoter sequence to be deleted. This plasmid was electroporated into the parental strain, JW710, and selected for kanamycin resistance and screened for 5FU sensitivity. The successful recombinant was designated as JW9293. For the construction of the subsequent $\mathrm{P}_{\text {sat }}$ mutants with alterations

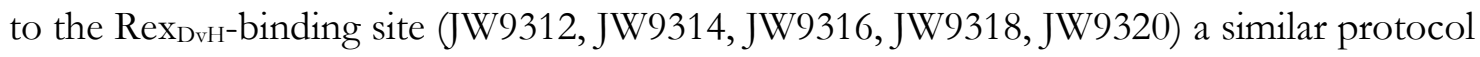
as (Parks et al. 2013) was followed. JW9293 was transformed with non-replicating plasmids with individual site-specific mutations in the $150 \mathrm{bp}$ fragment flanked by DNA homologous to that on either side of the integrated cassette. These $\mathrm{P}_{\text {sat }}$ mutant or restored strains were selected as $5-\mathrm{FU}^{\mathrm{r}}$ and screened for kanamycin sensitivity. The accuracy of the constructs was confirmed by sequencing both strands of a PCR amplified product across the mutation site. The deletion strain and promoter mutation strains that prevented sat transcription were expected not to grow with sulfate as electron acceptor and therefore $20 \mathrm{mM}$ sulfite was used in medium to recover these promoter mutations.

\section{Section 7. 3. 5. Rex protein expression cell-line}

A T7 promoter, amino terminal histidine tagged, rex expression plasmid (designated pMO3312) was constructed in E. coli. by the process of SLIC (Li et al. 2007) (Figure 7-3) from rex and the vector pET-14b. Primers used are listed in Table 7-3. The polymerase used for PCR was Herculase II Fusion DNA Polymerase. In brief, rex, without the translational start codon (ATG) was amplified by PCR from DvH gDNA (primers: DVU0916-expcloneF and DVU0916-expclone-R, 642 bp not including the additional overhangs added for 
cloning purposes). The majority of the pET-14b vector (not including 45 bases of the multicloning site) was amplified by PCR in two fragments. The first fragment contained the T7 promoter, ribosomal binding site, a 6x-histidine-tag, and a thrombin cut-site (primers: pET14b-1 and pET14b-22, 2387 bp), while the second fragment included the T7 terminator and an ampicillin resistance cassette (primers: pET14b-3 and pET14b-4, $2486 \mathrm{bp}$ ). The plasmid, pMO3312 (5268 bp), was sequenced across the insert with primers pET14b-seq-F and pET14b-seq-R and $1 \mathrm{ml}$ freezer stocks (10\% vol/vol glycerol) were made. A detailed description of protein expression and purification is described in a following section.

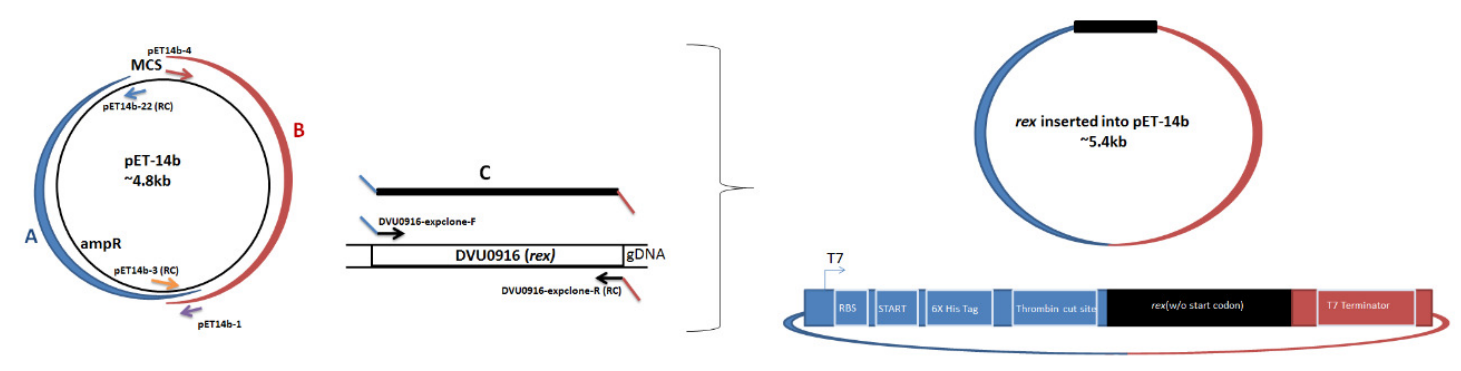

Figure 7-3: construction of pMO3312 (Rex expression plasmid). pMO3312 was generated by the process of SLIC (Li et al. 2007). Three fragments (A, B, C) were PCR amplified, A and B from pET14b, while C (containing the rex gene (without the start codon)) was amplified from genomic DNA. Primers for amplification of each fragment are shown as arrows. Overhanging regions are color coded to match where they align. Multicloning site (MCS); AmpR referes to antibiotic resistance marker for ampicillin; RC, reverse complement primer. The figure to the right is the constructed plasmid, pMO3312, color coded based on the three fragments used to generated the construct, with the T7 promoter identified. 
Table 7-3: Primer list for strains and plasmids generation

\begin{tabular}{|c|c|c|c|c|}
\hline Primer Name & Sequence & Length & Source & Descriptor \\
\hline \multicolumn{5}{|c|}{ General Primers } \\
\hline SpecRpUC-up & $\begin{array}{l}\text { GGGAAACGCCTGGTATCTTTATAG } \\
\text { TCCT }\end{array}$ & 28 & $\begin{array}{l}\text { (Parks et al. } \\
\text { 2013) }\end{array}$ & $\begin{array}{l}\text { For colony PCR, screen of and sequencing upstream region of deletion strains. } \\
\text { Forward primer. }\end{array}$ \\
\hline pMO719XbaI-Dn & TGGGTTCGTGCCTTCATCCG & 20 & $\begin{array}{l}\text { (Parks et al. } \\
\text { 2013) }\end{array}$ & $\begin{array}{l}\text { For colony PCR, screen of and sequencing upstream region of deletion strains. } \\
\text { Reverse primer. }\end{array}$ \\
\hline Kan-int-Fwd-rev-comp & СTCATCCTGTCTCTTGATCAGATCT & 25 & $\begin{array}{l}\text { (Parks et al. } \\
\text { 2013) }\end{array}$ & Sequencing Primer. Forward Primer. \\
\hline DvH-Upp gene Cterm-out & GCTGAAGCGCATCGTGGACAA & 21 & $\begin{array}{l}\text { (Parks et al. } \\
\text { 2013) }\end{array}$ & Sequencing Primer. Reverse Primer. \\
\hline pBG1-2199-F & GCTGAAAGCGAGAAGAGCGCAC & 22 & $\begin{array}{l}\text { (Parks et al. } \\
2013)\end{array}$ & Sequencing Primer. Forward Primer. \\
\hline SpecRpUC-F & CCAGCCAGGACAGAAATGCCTCG & 23 & $\begin{array}{l}\text { (Parks et al. } \\
\text { 2013) }\end{array}$ & $\begin{array}{l}\text { For amplification of } \mathrm{Sp}^{\mathrm{r}} \text { and pUC ori from pMO719 to make MED plasmid. } \\
\text { Used as overhang for SLIC. Forward Primer. }\end{array}$ \\
\hline SpecRpUC-R & $\begin{array}{l}\text { ATGTGAGCAAAAGGCCAGCAAAAG } \\
\text { GC }\end{array}$ & 26 & $\begin{array}{l}\text { (Parks et al. } \\
2013)\end{array}$ & $\begin{array}{l}\text { For amplification of } \mathrm{Sp}^{\mathrm{r}} \text { and } \mathrm{pUC} \text { ori from pMO719 to make MED plasmid. } \\
\text { Used as overhang for SLIC. Reverse Primer. }\end{array}$ \\
\hline KanPromNterm & CCGGAATTGCCAGCTGGG & 18 & $\begin{array}{l}\text { (Parks et al. } \\
\text { 2013) }\end{array}$ & $\begin{array}{l}\text { For amplification of } \mathrm{Km}^{\mathrm{r}} \text { from pMO719 to make MED plasmid. Used as } \\
\text { overhang for SLIC. Forward Primer. }\end{array}$ \\
\hline UPPCTerm & $\begin{array}{l}\text { CTTACTTGGTGCCGAATATCTTGTC } \\
\text { GC } \\
\end{array}$ & 27 & $\begin{array}{l}\text { (Parks et al. } \\
2013)\end{array}$ & $\begin{array}{l}\text { For amplification of } \mathrm{Km}^{\mathrm{r}} \text { from pMO719 to make MED plasmid. Used as } \\
\text { overhang for SLIC. reverse }\end{array}$ \\
\hline \multicolumn{5}{|c|}{ Protein Expression Strain } \\
\hline pET14b-1 & tcgecacctctgacttgagc & 20 & $\begin{array}{l}\text { (Christensen } \\
\text { et al. 2014) }\end{array}$ & $\begin{array}{l}\text { For amplification of a portion of pET14b with primer pET14b-22 to make } \\
\text { pMO3312. Forward Primer. }\end{array}$ \\
\hline pET14b-22 & catatggctgccgcgcggcaccagg & 25 & $\begin{array}{l}\text { (Christensen } \\
\text { et al. 2014) }\end{array}$ & $\begin{array}{l}\text { For amplification of a portion of pET14b with primer pET14b-1 to make } \\
\text { pMO3312. Reverse Primer. }\end{array}$ \\
\hline pET14b-3 & tcaggggataacgcaggaaagaacatg & 27 & $\begin{array}{l}\text { (Christensen } \\
\text { et al. 2014) }\end{array}$ & $\begin{array}{l}\text { For amplification of a portion of pET14b with primer pET14b-4 to make } \\
\text { pMO3312. Reverse Primer. }\end{array}$ \\
\hline pET14b-4 & getgctgccaccgctgagcaataa & 24 & $\begin{array}{l}\text { (Christensen } \\
\text { et al. 2014) }\end{array}$ & $\begin{array}{l}\text { For amplification of a portion of pET14b with primer pET14b-3 to make } \\
\text { pMO3312. Forward Primer. }\end{array}$ \\
\hline DVU0916-expclone-F & $\frac{\text { cctggtgccgcgcggcagccatatgACCAACATC }}{\text { AAAAGCGAACACATCCC }}$ & 51 & $\begin{array}{l}\text { (Christensen } \\
\text { et al. 2014) }\end{array}$ & $\begin{array}{l}\text { For amplification of DVU0916 (not including start codon) from gDNA with } \\
\text { primer DVU0916-expclone-R. Underlined portion used as overhang for SLIC } \\
\text { with fragment A (primer pET14b-22). Forward Primer. }\end{array}$ \\
\hline DVU0916-expclone-R & $\frac{\text { ttattgctcagcggtggcagcagcCTATTTGTTGC }}{\text { GCGAGAACGTGATGT }}$ & 50 & $\begin{array}{l}\text { (Christensen } \\
\text { et al. 2014) }\end{array}$ & $\begin{array}{l}\text { For amplification of DVU0916 (stop codon) from gDNA with primer } \\
\text { DVU0916-expclone-F. Underlined portion used as overhang for SLIC with } \\
\text { fragment B (primer pET14b-4). Reverse Primer. }\end{array}$ \\
\hline pET14b-seq-F & aagtggcgagcccgatcttc & 20 & $\begin{array}{l}\text { (Christensen } \\
\text { et al. 2014) }\end{array}$ & Sequencing primer located within pET14b in direction of insert. Forward. \\
\hline
\end{tabular}




\begin{tabular}{|c|c|c|c|c|}
\hline pET14b-seq-R & ctgctcgcttcgctacttgga & 21 & $\begin{array}{l}\text { (Christensen } \\
\text { et al. 2014) }\end{array}$ & Sequencing primer located within pET14b in direction of insert. Reverse \\
\hline \multicolumn{5}{|c|}{ Rex Marker-Exchange Deletions (MED) } \\
\hline DVU0916-1 & $\begin{array}{c}\text { GCCTTTTGCTGGCCTTTTGCTCACA } \\
\text { TGATGCTGAGAAGTTCGGTCCGAA } \\
\text { G }\end{array}$ & 50 & $\begin{array}{l}\text { (Korte et al. } \\
\text { 2014) }\end{array}$ & $\begin{array}{l}\text { For amplification of DVU0916 upstream region from gDNA with DVU0916-2 } \\
\text { primer to make pMO3311. Underlined portion used as overhang for SLIC } \\
\text { with Spr,pUC ori fragment (SpecRpUC-R). Amplification of Southern probe } \\
\text { for confirmation of DVU0916 deletion. Forward Primer. }\end{array}$ \\
\hline DVU0916-2 & $\begin{array}{l}\text { GCGACAAGATATTCGGCACCAAGT } \\
\text { AAGCGTTCGTTAACTTCACTTTTTG } \\
\text { CAATGCAC }\end{array}$ & 57 & $\begin{array}{l}\text { (Korte et al. } \\
\text { 2014) }\end{array}$ & $\begin{array}{l}\text { For amplification of DVU0916 upstream from gDNA with DVU0916-1 primer } \\
\text { to make pMO3311. Underlined portion used as overhang for SLIC with Kmr, } \\
\text { upp fragment (UppCterm). Amplification of Southern probe for confirmation } \\
\text { of DVU0916 deletion. Reverse Primer. }\end{array}$ \\
\hline DVU0916-3 & $\begin{array}{l}\text { GCGCCCCAGCTGGCAATTCCGG } \\
\text { CTGGAGCGTGAACGCCTCC }\end{array}$ & 42 & $\begin{array}{l}\text { (Korte et al. } \\
\text { 2014) }\end{array}$ & $\begin{array}{l}\text { For amplification of DVU0916 downstream from gDNA with DVU0916-4 to } \\
\text { make pMO3311. Underlined portion used as overhang for SLIC with Kmr, } \\
\text { upp fragment (KanPromNterm). Forward Primer. }\end{array}$ \\
\hline DVU0916-4 & $\begin{array}{c}\text { GTCGAGGCATTTCTGTCCTGGCTG } \\
\text { GGATTTCATGGGCCCCGATGTATT } \\
\text { GG }\end{array}$ & 50 & $\begin{array}{l}\text { (Korte et al. } \\
\text { 2014) }\end{array}$ & $\begin{array}{l}\text { For amplification of DVU0916 upstream region from gDNA with DVU0916-3 } \\
\text { primer to make pMO3311. Underlined portion used as overhang for SLIC } \\
\text { with Spr,pUC ori fragment (SpecRpUC-F). Reverse Primer. }\end{array}$ \\
\hline DVU0916-UP-int-F & CCTACGGCCAACGTCAACACCAAC & 23 & $\begin{array}{l}\text { (Korte et al. } \\
\text { 2014) }\end{array}$ & $\begin{array}{l}\text { Sequencing primer to confirm upstream region of deletion cassette of } \\
\text { pMO3311. Forward Primer. }\end{array}$ \\
\hline DVU0916-UP-int-R & GTTGGTGTTGACGTTGGCCGTAGG & 24 & $\begin{array}{l}\text { (Korte et al. } \\
\text { 2014) }\end{array}$ & $\begin{array}{l}\text { Sequencing primer to confirm upstream region of deletion cassette of } \\
\text { pMO3311. Reverse Primer. }\end{array}$ \\
\hline DVU0916-DWN-int-F & $\begin{array}{l}\text { GGATAGCGTGACATTCCCGGACGT } \\
\text { G }\end{array}$ & 25 & $\begin{array}{l}\text { (Korte et al. } \\
\text { 2014) }\end{array}$ & $\begin{array}{l}\text { Sequencing primer to confirm downstream region of deletion cassette of } \\
\text { pMO3311. Forward Primer. }\end{array}$ \\
\hline DVU0916-DWN-int-R & $\begin{array}{c}\text { CACGTCCGGGAATGTCACGCTATC } \\
\text { C }\end{array}$ & 25 & $\begin{array}{l}\text { (Korte et al. } \\
\text { 2014) }\end{array}$ & $\begin{array}{l}\text { Sequencing primer to confirm downstream region of deletion cassette of } \\
\text { pMO3311. Reverse Primer. }\end{array}$ \\
\hline \multicolumn{5}{|c|}{ Rex Marker-Less Deletions (MLD) } \\
\hline DVU0916-UP-MLD-R & $\begin{array}{l}\text { GGAGGCGTTCACGCTCCAGCGTTC } \\
\text { GTTAACTTCACTTTTTGCAATGCAC }\end{array}$ & 49 & This study & $\begin{array}{l}\text { For amplification of upstream fragment of DVU0916 with primer DVU0916-1 } \\
\text { to make pMO3319. Reverse Primer. }\end{array}$ \\
\hline DVU0916-DWN-MLD-F & $\begin{array}{c}\text { GTGCATTGCAAAAAGTGAAGTTAA } \\
\text { CGAACGCTGGAGCGTGAACGCCTC } \\
\text { C }\end{array}$ & 49 & This study & $\begin{array}{l}\text { For amplification of downstream fragment of DVU0916 with primer } \\
\text { DVU0916-4 to make pMO3319. Forward Primer. }\end{array}$ \\
\hline \multicolumn{5}{|c|}{ Complement } \\
\hline SLIC-DVU0916-comp-F & $\begin{array}{l}\text { AGGTTGGGAAGCCCTGCAATGCAG } \\
\text { TCCCAGGAGGTACCATATGACCAA } \\
\text { CATCAAAAGCGAACACATCC }\end{array}$ & 68 & $\begin{array}{l}\text { (Korte et al. } \\
\text { 2014) }\end{array}$ & $\begin{array}{l}\text { For amplification of DVU0916 to make pMO3313 complementation construct. } \\
\text { Underlined portion used as overhang for SLIC assembly with pMO9075 } \\
\text { fragment. Forward. }\end{array}$ \\
\hline SLIC-DVU0916-comp-R & $\begin{array}{c}\text { GATCGTGATCCCCTGCGCCATCAG } \\
\text { ATCCTTGCTATTTGTTGCGCGAGA } \\
\text { ACGTGATGTT } \\
\end{array}$ & 58 & $\begin{array}{l}\text { (Korte et al. } \\
\text { 2014) }\end{array}$ & $\begin{array}{l}\text { For amplification of DVU0916 to make pMO3313 complementation construct. } \\
\text { Underlined portion used as overhang for SLIC assembly with pMO9075 } \\
\text { fragment. reverse }\end{array}$ \\
\hline
\end{tabular}




\begin{tabular}{|c|c|c|c|c|}
\hline pMO9075-SLIC-F & CAAGGATCTGATGGCGCAGGG & 22 & $\begin{array}{c}\text { (Korte et al. } \\
\text { 2014) }\end{array}$ & $\begin{array}{l}\text { For amplification of pMO } 9075 \text { fragment for SLIC to make pMO3313 } \\
\text { complementation construct. forward }\end{array}$ \\
\hline pMO9075-SLIC-R3 & $\begin{array}{c}\text { CTGGGACTGCATTGCAGGGCTTCC } \\
\text { CAACCT } \\
\end{array}$ & 21 & $\begin{array}{c}\text { (Korte et al. } \\
2014)\end{array}$ & $\begin{array}{l}\text { For amplification of pMO9075 fragment for SLIC to make pMO3313 } \\
\text { complementation construct. reverse }\end{array}$ \\
\hline \multicolumn{5}{|c|}{ Sat Promoter Mutant strains } \\
\hline DVU1295-dProm-upF & $\begin{array}{l}\text { gccttttgctggccttttgctcacatCCTACCCGGC } \\
\text { AACGATGCG }\end{array}$ & 45 & $\begin{array}{l}\text { (Christensen } \\
\text { et al. 2014) }\end{array}$ & $\begin{array}{l}\text { For amplification of upstream fragment of DVU1295 with primer DVU1295- } \\
\text { dProm-upR to make pMO9292. DvH gDNA as template. Underlined portion } \\
\text { used as overhang for SLIC with fragment backbone (primer SpecRpUC-R). } \\
\text { Forward Primer. }\end{array}$ \\
\hline DVU1295-dProm-upR & $\begin{array}{l}\text { GCGACAAGATATTCGGCACCAAGT } \\
\underline{\text { AAGATGTCAAGCGGCAAAGCGCG }}\end{array}$ & 47 & $\begin{array}{l}\text { (Christensen } \\
\text { et al. 2014) }\end{array}$ & $\begin{array}{l}\text { For amplification of upstream fragment of DVU1295 with primer DVU1295- } \\
\text { dProm-upF to make pMO9292. DvH gDNA as template. Underlined portion } \\
\text { used as overhang for SLIC with fragment kanRUPP (primer UPPCTerm). } \\
\text { Reverse Primer. }\end{array}$ \\
\hline DVU1295-dProm-dnF & $\frac{\text { GCGCCCCAGCTGGCAATTCCGGAT }}{\text { GTCCAAGCTGGTTCCCGCTC }}$ & 44 & $\begin{array}{l}\text { (Christensen } \\
\text { et al. 2014) }\end{array}$ & $\begin{array}{l}\text { For amplification of downstream fragment (start codon of DVU1295) with } \\
\text { primer DVU1295-dProm-dnR to make pMO9292. DvH gDNA as template. } \\
\text { Underlined portion used as overhang for SLIC with fragment KanRUPP } \\
\text { (primer KanPromNTerm). Forward Primer. }\end{array}$ \\
\hline DVU1295-dProm-dnR & $\begin{array}{c}\text { cgaggcatttctgtcctggctGGACGAGAGCGA } \\
\text { TTTCCTGACC }\end{array}$ & 43 & $\begin{array}{l}\text { (Christensen } \\
\text { et al. 2014) }\end{array}$ & $\begin{array}{l}\text { For amplification of downstream fragment with primer DVU1295-dProm-DnF } \\
\text { to make pMO9292. DvH gDNA as template. Underlined portion used as } \\
\text { overhang for SLIC with fragment backbone (primerSpecRpUC-F). Reverse } \\
\text { Primer. }\end{array}$ \\
\hline DVU1295-upF & $\begin{array}{c}\text { ACCGTCGTACATGAGTCGGTTGAT } \\
\text { G }\end{array}$ & 25 & $\begin{array}{l}\text { (Christensen } \\
\text { et al. 2014) }\end{array}$ & $\begin{array}{l}\text { For amplification of sat promoter for confirmation of point mutations with } \\
\text { primer DVU1295-upR. Forward Primer. }\end{array}$ \\
\hline DVU1295-upR & $\begin{array}{c}\text { ACCTTACATCCTCCAGATGCGTGAT } \\
\text { G }\end{array}$ & 26 & $\begin{array}{l}\text { (Christensen } \\
\text { et al. 2014) }\end{array}$ & $\begin{array}{l}\text { For amplification of sat promoter for confirmation of point mutations with } \\
\text { primer DVU1295-upF. Reverse Primer. }\end{array}$ \\
\hline sat-ROP-R & $\frac{\text { CTTGTGAAAAAATTTACAAAatgtcaag }}{\text { cggcaaagcgc }}$ & 39 & $\begin{array}{l}\text { (Christensen } \\
\text { et al. 2014) }\end{array}$ & $\begin{array}{l}\text { For amplification of upstream fragment of DVU1295 with primer DVU1295- } \\
\text { dProm-upF to make pMO9311. Underlined portion used as overhang for } \\
\text { SLIC with fragment downstream (primer sat-ROP-F). Reverse Primer. }\end{array}$ \\
\hline sat-ROP-F & $\frac{\text { TTTGTAAATTTTTTCACAAGacggaatca }}{\text { acgcgacgec }}$ & 39 & $\begin{array}{l}\text { (Christensen } \\
\text { et al. 2014) }\end{array}$ & $\begin{array}{l}\text { For amplification of downstream fragment of DVU1295 with primer } \\
\text { DVU1295-dProm-dnR to make pMO9311. Underlined portion used as } \\
\text { overhang for SLIC with fragment upstream (primer sat-ROP-R). Forward } \\
\text { Primer }\end{array}$ \\
\hline sat-ROP-GtoA-R & $\frac{\text { CTTGTGAAAAAATTTATAAAatgtcaag }}{\text { cggcaaagcgc }}$ & 39 & $\begin{array}{l}\text { (Christensen } \\
\text { et al. 2014) }\end{array}$ & $\begin{array}{l}\text { For amplification of upstream fragment of DVU1295 with primer DVU1295- } \\
\text { dProm-upF to make pMO9313. Underlined portion used as overhang for } \\
\text { SLIC with fragment downstream (primer sat-ROP-GtoA-F). Reverse Primer }\end{array}$ \\
\hline sat-ROP-GtoA-F & $\frac{\text { TTTATAAATTTTTTCACAAGacggaatca }}{\text { acgcgacgcc }}$ & 39 & $\begin{array}{l}\text { (Christensen } \\
\text { et al. 2014) }\end{array}$ & $\begin{array}{l}\text { For amplification of downstream fragment of DVU1295 with primer } \\
\text { DVU1295-dProm-dnR to make pMO9313. Underlined portion used as } \\
\text { overhang for SLIC with fragment upstream (primer sat-ROP-GtoA-R). } \\
\text { Forward Primer }\end{array}$ \\
\hline
\end{tabular}




\begin{tabular}{|c|c|c|c|c|}
\hline sat-ROP-IR1-R & $\frac{\text { CTTGTGAAAAAATTCGTAAAatgtcaag }}{\text { cggcaaagcgc }}$ & 39 & $\begin{array}{l}\text { (Christensen } \\
\text { et al. 2014) }\end{array}$ & $\begin{array}{l}\text { For amplification of upstream fragment of DVU1295 with primer DVU1295- } \\
\text { dProm-upF to make pMO9315. Underlined portion used as overhang for } \\
\text { SLIC with fragment downstream (primer sat-ROP-IR1-F). Reverse Primer }\end{array}$ \\
\hline sat-ROP-IR1-F & $\frac{\text { TTTACGAATTTTTTCACAAGacggaatca }}{\text { acgcgacgcc }}$ & 39 & $\begin{array}{l}\text { (Christensen } \\
\text { et al. 2014) }\end{array}$ & $\begin{array}{l}\text { For amplification of downstream fragment of DVU1295 with primer } \\
\text { DVU1295-dProm-dnR to make pMO9315. Underlined portion used as } \\
\text { overhang for SLIC with fragment upstream (primer sat-ROP-IR1-R). Forward } \\
\text { Primer }\end{array}$ \\
\hline sat-ROP-IR2-R & $\frac{\text { CTTACAAAAAAATTTACAAAatgtcaagc }}{\text { ggcaaagcgc }}$ & 39 & $\begin{array}{l}\text { (Christensen } \\
\text { et al. 2014) }\end{array}$ & $\begin{array}{l}\text { For amplification of upstream fragment of DVU1295 with primer DVU1295- } \\
\text { dProm-upF to make pMO9317. Underlined portion used as overhang for } \\
\text { SLIC with fragment downstream (primer sat-ROP-IR2-F). Reverse Primer }\end{array}$ \\
\hline sat-ROP-IR2-F & $\frac{\text { TTTGTAAATTTTTTTGTAAGacggaatc }}{\text { aacgcgacgcc }}$ & 39 & $\begin{array}{l}\text { (Christensen } \\
\text { et al. 2014) }\end{array}$ & $\begin{array}{l}\text { For amplification of downstream fragment of DVU1295 with primer } \\
\text { DVU1295-dProm-dnR to make pMO9317. Underlined portion used as } \\
\text { overhang for SLIC with fragment upstream (primer sat-ROP-IR2-R). Forward } \\
\text { Primer }\end{array}$ \\
\hline sat-ROP-IR1and2-R & $\frac{\text { CTTACAAAAAAATTCGTAAAatgtcaag }}{\text { cggcaaagcgc }}$ & 39 & $\begin{array}{l}\text { (Christensen } \\
\text { et al. 2014) }\end{array}$ & $\begin{array}{l}\text { For amplification of upstream fragment of DVU1295 with primer DVU1295- } \\
\text { dProm-upF to make pMO9319. Underlined portion used as overhang for } \\
\text { SLIC with fragment downstream (primer sat-ROP-IR1and2-F). Reverse } \\
\text { Primer }\end{array}$ \\
\hline sat-ROP-IR1 and2-F & $\frac{\text { TTTACGAATTTTTTTGTAAGacggaatc }}{\text { aacgcgacgcc }}$ & 39 & $\begin{array}{l}\text { (Christensen } \\
\text { et al. 2014) }\end{array}$ & $\begin{array}{l}\text { For amplification of downstream fragment of DVU1295 with primer } \\
\text { DVU1295-dProm-dnR to make pMO9319. Underlined portion used as } \\
\text { overhang for SLIC with fragment upstream (primer sat-ROP-IR1and2-R). } \\
\text { Forward Primer }\end{array}$ \\
\hline
\end{tabular}




\section{Section 7.4. $\quad \operatorname{Rex}_{\mathrm{DvH}}$ protein purification}

To obtain Rex $\mathrm{DvH}_{\mathrm{v}}$, a 6XHis tag was added cloning rex into pET-14b (Novagen, Madison, WI) (Figure 7-3) and transformed into BL21(DE3) competent cells (Agilent). After induction with isopropyl-beta-D-thiogalactopyranoside (Gold Biotechnologies ${ }^{\circledR}$ ) the protein was purified by the His60 Ni Gravity Column Purification Kit (Clontech, Mountain View, CA). In detail, 50 ng of pMO3312 was transformed, according to the manufacturer's protocol, plated on LC + ampicillin $(100 \mu \mathrm{g} / \mathrm{ml})$ and incubated overnight at $37^{\circ} \mathrm{C}$. Individual isolates were PCR screened for plasmid (primers: pET14b-seq-F and pET14b-seq-R) and one correctly identified isolate was grown in $10 \mathrm{ml} \mathrm{LC}+$ ampicillin $(100 \mu \mathrm{g} / \mathrm{ml})$ overnight at $37^{\circ} \mathrm{C}$ and confirmed for the correct sequence. From the overnight culture, $5 \mathrm{ml}$ was transferred to $95 \mathrm{ml} \mathrm{LC} \mathrm{(no} \mathrm{antibiotics)} \mathrm{and} \mathrm{incubated} \mathrm{at} 26^{\circ} \mathrm{C}$ with shaking for 2.5 hours. Half of the culture $(50 \mathrm{ml})$ was removed and frozen $\left(-20^{\circ} \mathrm{C}\right)$ to be used as a non-induced (NI) control. IPTG (Gold Biotechnologies ${ }^{\circledR}$ ) at $1 \mathrm{mM}$ final concentration was added to the remaining $50 \mathrm{ml}$ and then incubated at $26^{\circ} \mathrm{C}$ with shaking for an additional 2.5 hours, induced sample (I). Next, $45 \mathrm{ml}$ of NI and I culture were spun down $\left(4^{\circ} \mathrm{C}, 4150 \mathrm{rpm}, 12\right.$ min) and the pellet was stored on ice. His-Rex protein was purified with the His60 Ni Gravity Column Purification Kit according to the manufacturer's protocol. The cell pellets were resuspended in xTractor Buffer (2 ml per $100 \mathrm{mg}$ ) and supplemented with TURBO ${ }^{\mathrm{TM}}$ DNase (2 U/100 mg pellet; Ambion), 1 mM phenylmethanesulfonyl fluoride (PMSF, $0.2 \mathrm{M}$ stock in isopronanol) (Sigma-Aldrich)) and a protease inhibitor (Pierce Halt ${ }^{\mathrm{TM}}$ Protease Inhibitor Cocktail, EDTA-Free) (Thermo Fisher Scientific). The samples were incubated on ice for $15 \mathrm{~min}$, mixing occasionally and then centrifuged for $20 \mathrm{~min}$ at $10,000 \mathrm{xg}$ at $4^{\circ} \mathrm{C}$. The supernatant was collected and stored on ice until sample preparation. While the samples were being centrifuged two columns were equilibrated with 10 bed volumes $(1 \mathrm{ml}=1 \mathrm{bed}$ 
volume) of His60 Ni Equilibration Buffer. Protein sample (4 ml) was added to the column, resuspended with the resin and rotated for $1 \mathrm{hr}$ at $4^{\circ} \mathrm{C}$. The columns were allowed to settle, rinsed with $10 \mathrm{ml}$ His60 Ni Equilibration Buffer, followed by $10 \mathrm{ml} \mathrm{His60} \mathrm{Wash} \mathrm{Buffer,} \mathrm{and}$ eluted with $10 \mathrm{ml}$ His60 Ni Elution Buffer, collecting 1-ml fractions and analyzed for protein (Noble et al. 2009) (Figure 7-4). Samples with the greatest concentration were pooled together. The pooled sample was passed through a PD-10 desalting column (GE Healthcare Bio-Sciences, Piscataway, NJ) according to the manufacturer's protocol and analyzed by Bradford (Noble et al. 2009) or Qubit® Protein Assay Kit. Several $100 \mu$ l aliquots were made and stored at $-20^{\circ} \mathrm{C}$. An SDS-PAGE was performed on the purified protein to ensure purity of the Rex $\mathrm{DvvH}_{\mathrm{vH}}$ monomer $(\sim 25 \mathrm{kDa}$ ) (Figure $7-4)$. Each sample was mixed with an equal volume of $2 \mathrm{X}$ SDS loading dye and loaded to a 4-20\% wt/vol Tris-Glycine-SDS polyacrylamide gel (Bio-Rad), and electrophoresed at $200 \mathrm{~V}$ (50 mAmps) for $30 \mathrm{~min}$ in 1X Tris-Glycine-SDS Buffer (Bio-Rad). The gel was then stained for $1 \mathrm{hr}$ with $25 \mathrm{ml}$ coomassie blue (per 2 liters: 2 g coomassie BB G-250, $140 \mathrm{ml}$ acetic acid, $1000 \mathrm{ml}$ methanol, $860 \mathrm{ml}$ water, filter sterilized) and destained (140 $\mathrm{ml}$ acetic acid, $400 \mathrm{ml}$ methanol, $60 \mathrm{ml}$ glycerol, $1400 \mathrm{ml}$ water) overnight. The presence of NADH bound to Rex $\operatorname{DvH}_{\mathrm{DH}}$ was checked by measuring $A_{340}$ to ensure purity of the protein from additional co-factors (Figure 7-5). The protocol by Brekasis and Paget (Brekasis et al. 2003) was used for DNA

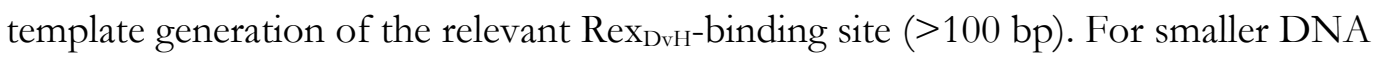
fragments (40 bp), reverse complemented primers were annealed following previous protocols (Sigma-Aldrich). These sequences were 5'-end labeled with T4 Polynucleotide Kinase (Promega) and $\left[\gamma^{32} \mathrm{P}\right]$ ATP (Perkin Elmer, Waltham MA) according to the manufacturer's protocol. The unlabeled nucleotides were removed by QIAquick Nucleotide Removal Kit (Qiagen, Valencia CA). 

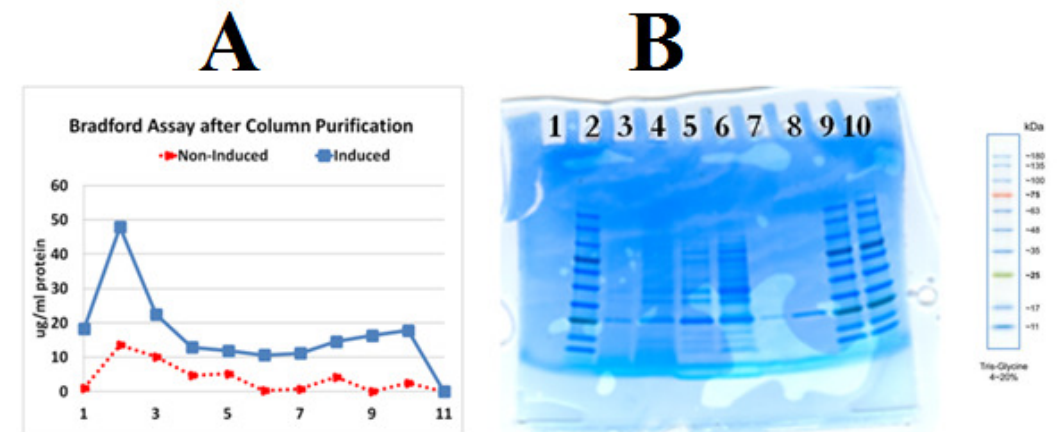

Figure 7-4: Protein purification of 6XHis-Rex (Rex $\left.\mathbf{D}_{\mathrm{DvH}}\right)$. A) Elution of protein samples during protein purification in 1-ml fractions. Protein concentration $(\mu \mathrm{g} / \mathrm{ml})$ is plotted versus fraction. Fractions 1-3 were pooled together and passed over a desalting column. B) SDS-PAGE, stained with Coomassie Brilliant Blue G-250, of the purification process for 6XHis-Rex. Non-Induced (NI). Induced (I). Lanes as follows: (1) Empty, (2) Ladder, (3) Whole cell extract (NI), (4) Whole cell extract (I), (5) Lysed cell extract (NI), (6) Lysed cell extract (I), (7) After column purification (NI), (8) After column purification (I), (9) Ladder, (10) Ladder. Protein ladder is BLUEstain ${ }^{\mathrm{TM}} 3$ (Gold Biotechnologies $($ ) $)$ Expected size of Rex monomer is $\sim 25 \mathrm{kDa}$.

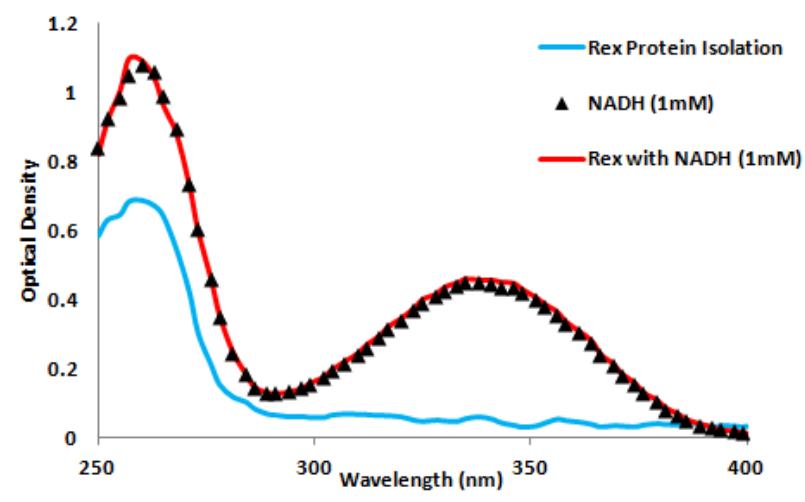

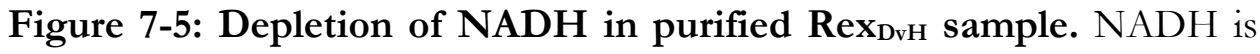
detected at $\mathrm{A}_{340}$. Purified His-Rex $\mathrm{x}_{\mathrm{DvH}}$ protein (blue) is absent of $\mathrm{A}_{340}$ peak and therefore, does not contain NADH. 


\section{Section 7.5. Rex operon determination by RT-PCR}

Samples to be used for Reverse-Transcriptase PCR (RT-PCR) were initially collected from

DNase-treated RNA samples that were stored at $-80^{\circ} \mathrm{C}$. A $5 \mu \mathrm{l}$ aliquot $(20 \mathrm{ng} / \mu \mathrm{l})$ of DNasetreated RNA was reverse transcribed to single stranded cDNA via iScript ${ }^{\mathrm{TM}} \mathrm{cDNA}$ synthesis kit (Bio-Rad, Hercules, CA). The amplification protocol is as follows: in a $20 \mu \mathrm{l}$ reaction, $13.5 \mu \mathrm{l}$ water, $1 \mu \mathrm{l}$ template (water, gDNA, mRNA, or cDNA), $2 \mu \mathrm{l} 10 \mathrm{X}$ Taq Polymerase Buffer, $1 \mu \mathrm{l}$ dNTP mix (each dNTP at $1.5 \mathrm{mM}$ ), $1 \mu$ l each of specified Forward and Reverse primers (Table 7-4) and $0.5 \mu \mathrm{l}$ Taq DNA Polymerase (NEB). In a thermocycler, 50 cycles of amplification were carried out $\left(20 \mathrm{sec}\right.$ at $94^{\circ} \mathrm{C}, 20 \mathrm{sec}$ at $57^{\circ} \mathrm{C}$, and $90 \mathrm{sec}$ at $\left.72^{\circ} \mathrm{C}\right)$ followed by a final extension for $5 \mathrm{~min}$ at $72^{\circ} \mathrm{C}$. A $10-\mu \mathrm{l}$ aliquot of each product was electrophoresed (50 min at $110 \mathrm{~V}$ ) on a $0.8 \% \mathrm{wt} / \mathrm{vol}$ agarose gel and analyzed for a single product.

Table 7-4: Primer list for Real-Time PCR (RT-PCR)

\begin{tabular}{|c|c|c|c|c|}
\hline Name & Sequence $\left(5^{\prime}-3^{\prime}\right)$ & length & Source & Descriptor \\
\hline DVU0847-3F & $\begin{array}{c}\text { TGCTCGGTTCGCA } \\
\text { CTCCG }\end{array}$ & 18 & $\begin{array}{l}\text { (Zane et al. } \\
\text { 2010) }\end{array}$ & $\begin{array}{l}\text { For amplification of aps } A \text { with primer } \\
\text { DVU0847-4R for DNA contamination } \\
\text { screen of RNA sample and RT-PCR. } \\
\text { Forward primer. }\end{array}$ \\
\hline DVU0847-4R & $\begin{array}{l}\text { GGTGTAGTAGGTA } \\
\text { CCCACGCCA }\end{array}$ & 22 & $\begin{array}{l}\text { (Zane et al. } \\
\text { 2010) }\end{array}$ & $\begin{array}{l}\text { For amplification of aps } A \text { with primer } \\
\text { DVU0847-3F for DNA contamination } \\
\text { screen and RT-PCR. Reverse primer. }\end{array}$ \\
\hline DVU0917-int-F & $\begin{array}{c}\text { CTCTGGGTCTCACC } \\
\text { TGCCTC }\end{array}$ & 20 & This study & $\begin{array}{l}\text { For amplification of DVU0917 (atpE) with } \\
\text { primer DVU0917-int-R for analysis by RT- } \\
\text { PCR. Template is from DvH gDNA, } \\
\text { mRNA, and cDNA. Forward primer }\end{array}$ \\
\hline DVU0917-int-R & $\begin{array}{l}\text { GGTTGGCGAACAG } \\
\text { GAGGATC }\end{array}$ & 20 & This study & $\begin{array}{l}\text { For amplification of DVU0917 (atpE) with } \\
\text { primer DVU0917-int-F for analysis by RT- } \\
\text { PCR. Template is from DvH gDNA, } \\
\text { mRNA, and cDNA. Reverse primer. }\end{array}$ \\
\hline DVU0916-int-F & $\begin{array}{l}\text { CTACTACGTCAAGA } \\
\text { GTCTCATCGAATCC }\end{array}$ & 29 & This study & $\begin{array}{l}\text { For amplification of DVU0916 (rex) with } \\
\text { primer DVU0916-int-R for analysis by RT- } \\
\text { PCR. Template is from DvH gDNA, } \\
\text { mRNA, and cDNA. Forward primer. }\end{array}$ \\
\hline DVU0916-int-R & $\begin{array}{l}\text { CGTAGAGATGGTG } \\
\text { GAAGAAGTCC }\end{array}$ & 23 & This study & $\begin{array}{l}\text { For amplification of DVU0916 (rex) with } \\
\text { primer DVU0916-int-F for analysis by RT- } \\
\text { PCR. Template is from DvH gDNA, } \\
\text { mRNA, and cDNA. Reverse primer. }\end{array}$ \\
\hline DVU0915-int-F & $\begin{array}{l}\text { GCTTCGTGCTTCTG } \\
\text { ACCGATTTC }\end{array}$ & 23 & This study & $\begin{array}{l}\text { For amplification of DVU0915 with primer } \\
\text { DVU0915-int-R for analysis by RT-PCR. } \\
\text { Template is from DvH gDNA, mRNA, } \\
\text { and cDNA. Forward primer. }\end{array}$ \\
\hline DVU0915-int-R & $\begin{array}{l}\text { GACATGAAGGATG } \\
\text { CGGGCTAC }\end{array}$ & 21 & This study & $\begin{array}{l}\text { For amplification of DVU0915 with primer } \\
\text { DVU0915-int-F for analysis by RT-PCR. } \\
\text { Template is from DvH gDNA, mRNA, } \\
\text { and cDNA. Reverse primer. }\end{array}$ \\
\hline
\end{tabular}




\section{Section 7.6. mRNA expression quantification by qRT-PCR}

Initial transcript expression of aps $B A$ was determined by northern blot as described by Zane et al. (2010). Refer to Chapter 5 for discussion. Subsequent analyses for all samples and conditions were performed by qRT-PCR as discussed in the following section.

\section{Section 7. 6. 1. Primer design, protocol and analysis}

Primers to be used for qRT-PCR were manually designed to be 18-30 bp in length, have a GC-content $\sim 50 \%, \mathrm{~T}_{\mathrm{m}}=58-60^{\circ} \mathrm{C}$, amplify a $\sim 100-150 \mathrm{bp}$ region at the $3^{\prime}$-end of the transcript for each gene (Table 7-5). Additional discussion is covered in Chapter 5. qPCRs were set up according to the manufacturer's protocol with SsoFast ${ }^{\mathrm{TM}}$ EvaGreen ${ }^{\circledR}$ Supermix (Bio-Rad) on a CFX96 ${ }^{\mathrm{TM}}$ and analyzed with CFX Manager ${ }^{\mathrm{TM}}$ (version 3.1, Bio-Rad), with the curve fit to regression (Pfaffl 2001; Vandesompele et al. 2002; Hellemans et al. 2007). The amplification protocol was initiated with a 3 min pre-incubation at $95^{\circ} \mathrm{C}$ and processed through 40 cycles of denaturation $\left(30 \mathrm{sec}\right.$ at $\left.95^{\circ} \mathrm{C}\right)$ and annealing/extension $\left(30\right.$ sec at $\left.65^{\circ} \mathrm{C}\right)$. The fluorescent signal was acquired at the end of each annealing/extension step. The melt curve protocol included annealing at the annealing/extension temperature $\left(65^{\circ} \mathrm{C}\right)$ and melting at a ramp rate of $0.5^{\circ} \mathrm{C} / 5 \sec$ up to $95^{\circ} \mathrm{C}$, with the fluorescent signal acquired continuously during the melt curve. Additional discussion is provided in the appendix. 
Table 7-5: Primers list for quantitative Real-Time PCR (qRT-PCR)

\begin{tabular}{|c|c|c|c|c|}
\hline Name & Sequence (5'-3’) & bp & Source & Gene \\
\hline DVU0173-qPCR-F1 & CGACCAGGACCTCATGCC & 18 & This study & \multirow{2}{*}{$\operatorname{phs} A$} \\
\hline DVU0173-qPCR-R & СTACCTGACCCCTGCTTTGC & 20 & This study & \\
\hline DVU0402-qPCR-F1 & CAGAAGCTGCTCGAAGTGACCGAAAT & 26 & (Christensen et al. 2014) & \multirow{2}{*}{$d s r A$} \\
\hline DVU0402-qPCR-R1 & ATCTCAGGTGTCTCTTGCGGTATTCC & 26 & (Christensen et al. 2014) & \\
\hline DVU0414-qPCR-F & GCGTGTCATCGAGTGGGTC & 19 & This study & \multirow{2}{*}{ tme } \\
\hline DVU0414-qPCR-R1 & CGTAGCTGTCGATGAAGGCC & 20 & This study & \\
\hline DVU0835-qPCR-F & TGTCTTCCCCСTGCACTCG & 19 & (Christensen et al. 2014) & \multirow{2}{*}{$r p l S$} \\
\hline DVU0835-qPCR-R1 & CTTGATGCGGGCAGCCTTAC & 20 & (Christensen et al. 2014) & \\
\hline DVU0847-qPCR-F3 & ACTCCAAGTGGAAGTGCTTCGTGAA & 25 & (Christensen et al. 2014) & \multirow{2}{*}{$\operatorname{aps} A$} \\
\hline DVU0847-qPCR-R3 & CTATTCGGGGATGATCTGGTAGTAGG & 26 & (Christensen et al. 2014) & \\
\hline DVU0848-qPCR-F2 & CGTTCGATGTACCTGTCGATGAAGAC & 26 & This study & \multirow{2}{*}{$q m o A$} \\
\hline DVU0848-qPCR-R1 & GCAGAGCGCATGACATCAAGG & 21 & This study & \\
\hline DVU0851-qPCR-F2 & GAAATCGAAAGCAGCGTGAAGGACAT & 26 & This study & \multirow{2}{*}{$q m o D$} \\
\hline DVU0851-qPCR-R2 & ACGCCACCGTCGGAAGA & 17 & This study & \\
\hline DVU0915-qPCR-F2 & GCTTTTATGCGGAAGTCAGGGATACTG & 27 & This study & \multirow{2}{*}{$\begin{array}{c}\text { DVU0 } \\
915\end{array}$} \\
\hline DVU0915-qPCR-R1 & CGCTATCCCCCGTGTGC & 17 & This study & \\
\hline DVU0916-qPCR-F1 & GGCATCAAGGGCATTCTCAACTAC & 24 & (Christensen et al. 2014) & \multirow{2}{*}{ rex } \\
\hline DVU0916-qPCR-R2 & CTATTTGTTGCGCGAGAACGTGAT & 24 & (Christensen et al. 2014) & \\
\hline DVU1290-qPCR-F2 & CGCAACCTTCCGAACAACTCC & 21 & This study & \multirow{2}{*}{$d s r M$} \\
\hline DVU1290-qPCR-R & TTACTCGGCAGCAGCTTCGT & 20 & This study & \\
\hline DVU1295-qPCR-F2 & GCAAGGCCCTTTCCGAAGG & 19 & (Christensen et al. 2014) & \multirow{2}{*}{ sat } \\
\hline DVU1295-qPCR-R & TTACATCACAGAGCCGGAAGCG & 22 & (Christensen et al. 2014) & \\
\hline DVU1311-qPCR-F & AACTGTTCAATCTGCGCTTCCGT & 23 & (Christensen et al. 2014) & \multirow{2}{*}{$r p m C$} \\
\hline DVU1311-qPCR-R & СТАTTCСTTTTCCTTCAGAATGGTCTGAATC & 32 & (Christensen et al. 2014) & \\
\hline DVU1636-qPCR-F1 & GTTCCTGCTGCTTACCGACATC & 22 & This study & \multirow{2}{*}{$p p a C$} \\
\hline DVU1636-qPCR-R1 & GCTTCTTGCGGCTCATCACG & 20 & This study & \\
\hline DVU1932-qPCR-F & CCACGACATCTACTACAACACCG & 23 & This study & \multirow{2}{*}{$a d k$} \\
\hline DVU1932-qPCR-R & CTAGGAAAGCTGTGCGAGGAG & 21 & This study & \\
\hline DVU2776-qPCR-F & AACGGTATCGCTCCGATGGTTC & 22 & This study & \multirow{2}{*}{$d s r C$} \\
\hline DVU2776-qPCR-R2 & AGGCCGGCCATCTTGCA & 17 & This study & \\
\hline DVU2791-qPCR-F2 & CAGTGCAAGCTGCCAGACC & 19 & This study & \multirow{2}{*}{$d b c A$} \\
\hline DVU2791-qPCR-R & GGCCCTTGCCСTCСТTCT & 18 & This study & \\
\hline DVU2792-qPCR-F1 & GCGGACTCTGCGGGTAC & 17 & This study & \multirow{2}{*}{$m f C$} \\
\hline DVU2792-qPCR-R & CGAGCTTCTGCTTCGAGAGACG & 22 & This study & \\
\hline DVU3262-qPCR-F & TCGCCACATGGCAGGAG & 17 & This study & \multirow{2}{*}{$f d r A$} \\
\hline DVU3262-qPCR-R2 & AGACGGCCTCCTCGGAAG & 18 & This study & \\
\hline DVU3264-qPCR-F & CCATCAACGCTCTCGGCATC & 20 & This study & \multirow{2}{*}{$\begin{array}{c}\text { DVU3 } \\
264\end{array}$} \\
\hline DVU3264-qPCR-R & TCAGAGCGTCACСTCCСC & 18 & This study & \\
\hline DVU3360-qPCR-F & GTCAGCGGGACAGAACAGAAG & 21 & This study & \multirow{2}{*}{ parB } \\
\hline DVU3360-qPCR-R & TTAGTGGTTGGAAAGCCCAAGGC & 23 & This study & \\
\hline DORF6830-qPCR-F1 & GAAGATGTGGCAGTGCGAGC & 20 & This study & \multirow{2}{*}{$\operatorname{qrc} A$} \\
\hline DORF6830-qPCR-R & TTACTTGTGGCACACGAAGCAG & 22 & This study & \\
\hline
\end{tabular}




\section{Section 7. 6. 2. Reference gene determination}

Genes to be used as internal (reference) controls were $r p l S$ (DVU0835) and $r p m C$

(DVU1311) (Zhou et al. 2010) because they are expressed at similar levels to the genes to be assessed in this study and their expression levels were not found to change during exposure to environmental stresses (http://www.microbesonline.org/). To validate these reference genes, the strategy by Hellemans and coworkers was implemented (Hellemans et al. 2007) and the genes were shown to have minimal transcriptional differences among the strains, mutants and conditions tested (Mean Coefficient of Variance $(\mathrm{CV}<0.25)$ and Mean $\mathrm{M}<0.5)$ (data not shown).

\section{Section 7. 6.3. Sample preparation for transcript analysis}

Samples to be used for quantitative Reverse Transcriptase PCR (qRT-PCR) were initially collected from DNase-treated RNA samples that were stored at $-80^{\circ} \mathrm{C}$. Purity of RNA from DNA is confirmed by a PCR screen with primers DVU0847-3F and DVU0847-4R (Figure 7-1). A $5 \mu \mathrm{l}$ aliquot $(20 \mathrm{ng} / \mu \mathrm{l})$ of DNase-treated RNA was reverse transcribed to single stranded cDNA via iScript ${ }^{\mathrm{TM}}$ cDNA synthesis kit (Bio-Rad, Hercules, CA). For each gene to be analyzed, a standard curve (6 logs, serial dilution from $100 \mathrm{ng} / \mu \mathrm{l}$ stock cDNA) was performed to calculate efficiency. For each transcript the relative abundance was normalized to the reference gene transcripts $(r p l S$ and $r p m C)$ in the specified sample. 


\section{Section 7. 7. in vitro protein-DNA interaction studies}

$\operatorname{Re}_{\mathrm{DvH}}$ protein was isolated as described previously in Section 7. 4.

\section{Section 7. 7. 1. Electrophoretic Mobility Shift Assay (EMSA)}

EMSAs were performed according to Ravcheev et al. (Ravcheev et al. 2012). Primers used for generating dsDNA fragment are listed in Table 7-6. In brief, dsDNA fragments $(0.1 \mathrm{nM})$ were incubated with $\operatorname{Rex}_{\mathrm{DvH}}$ at specified concentrations $(0-2000 \mathrm{nM})$ in a final volume of 30 $\mu$ l. The binding buffer contained $20 \mathrm{mM}$ Tris- $\mathrm{HCl} \mathrm{pH} 8.0,10 \%$ vol/vol glycerol, $1 \mathrm{mM}$ $\mathrm{MgCl}_{2}$ and $40 \mathrm{mM} \mathrm{KCl}$. Samples were incubated at $37^{\circ} \mathrm{C}$ for $25 \mathrm{~min}$, placed on ice for $2 \mathrm{~min}$ and then separated $\left(90 \mathrm{~V}, 70 \mathrm{~min}, 4^{\circ} \mathrm{C}\right)$ on a $5 \%$ (wt/vol) native Tris-Borate-EDTA (TBE) polyacrylamide gel that was preincubated $\left(200 \mathrm{~V}, 30\right.$ min at $\left.4^{\circ} \mathrm{C}\right)$ in $0.5 \mathrm{X}$ TBE buffer (BioRad). In the cases when pyridine nucleotides were examined, desired concentrations were added after the initial incubation and were incubated for an additional $10 \mathrm{~min}$ at $37^{\circ} \mathrm{C}$. The gel was removed from the apparatus, wrapped in plastic wrap and exposed to a Kodak Imaging Screen K (Bio-Rad) typically 15-60 min in a sealed cassette, followed by imaging with Personal Molecular Imager ${ }^{\mathrm{TM}}$ (PMI) (Bio-Rad). 
Table 7-6: Primers list for Electrophoretic Mobility Shift Assay (EMSA)

\begin{tabular}{|c|c|c|c|c|}
\hline Name & Sequence $\left(5^{\prime}-3^{\prime}\right)$ & bp & Source & Descriptor \\
\hline $\begin{array}{c}\text { DVU1295-UP-F- } \\
\text { EMSA }\end{array}$ & AGCGAAATGTGCCCGCCA & 18 & $\begin{array}{l}\text { (Christensen et } \\
\text { al. 2014) }\end{array}$ & $\begin{array}{l}\text { For amplification of promoter sequence of sat with primer DVU1295-200-R- } \\
\text { EMSA (fragment ALL, or promoter mutation) or DVU1295-UP-R-EMSA } \\
\text { (fragment UP). Forward primer. }\end{array}$ \\
\hline $\begin{array}{c}\text { DVU1295-200-R- } \\
\text { EMSA }\end{array}$ & АССТTACATCCTCCAGATGCGTGA & 24 & $\begin{array}{c}\text { (Christensen et } \\
\text { al. 2014) }\end{array}$ & $\begin{array}{l}\text { For amplification of promoter sequence of sat with primer DVU1295-UP-F- } \\
\text { EMSA (fragment ALL or promoter mutation) or DVU1295-DWN-F- } \\
\text { EMSA (fragment DOWN). Reverse primer }\end{array}$ \\
\hline $\begin{array}{l}\text { DVU1295-UP-R- } \\
\text { EMSA }\end{array}$ & ATGTCAAGCGGCAAAGCGCG & 20 & $\begin{array}{l}\text { (Christensen et } \\
\text { al. 2014) }\end{array}$ & $\begin{array}{l}\text { For amplification of promoter sequence of sat with primer DVU1295-UP-F- } \\
\text { EMSA (fragment UP). Reverse primer. }\end{array}$ \\
\hline $\begin{array}{l}\text { DVU1295-DWN-F- } \\
\text { EMSA }\end{array}$ & ACGGAATCAACGCGACGCCA & 20 & $\begin{array}{l}\text { (Christensen et } \\
\text { al. 2014) }\end{array}$ & $\begin{array}{l}\text { For amplification of promoter sequence of sat with primer DVU1295-200-R- } \\
\text { EMSA (fragment DOWN). Forward primer. }\end{array}$ \\
\hline $\begin{array}{l}\text { DVU1295-RegPLUS- } \\
\text { F-EMSA }\end{array}$ & $\begin{array}{l}\text { CGCTTGACATTTTGTAAAATTTT'TTC } \\
\text { ACAAGACGGAATCAA }\end{array}$ & 40 & $\begin{array}{l}\text { (Christensen et } \\
\text { al. 2014) }\end{array}$ & $\begin{array}{l}\text { Rex binding site upstream of sat. Annealed with DVU1295-RegPLUS-R-EMSA } \\
\text { (fragment RegPLUS). Forward primer }\end{array}$ \\
\hline $\begin{array}{l}\text { DVU1295-RegPLUS- } \\
\text { R-EMSA }\end{array}$ & $\begin{array}{l}\text { TTGATTCCGTCTTGTGAAAAAATT } \\
\text { TACAAAATGTCAAGCG }\end{array}$ & 40 & $\begin{array}{l}\text { (Christensen et } \\
\text { al. 2014) }\end{array}$ & $\begin{array}{l}\text { Rex binding site upstream of sat. Annealed with DVU1295-RegPLUS-F-EMSA } \\
\text { (fragment RegPLUS). Reverse primer }\end{array}$ \\
\hline $\begin{array}{l}\text { DVU1295-RegPLUS- } \\
\text { GtoA-F }\end{array}$ & $\begin{array}{l}\text { CGCTTGACATTTTATAAATTTTTTC } \\
\text { ACAAGACGGAATCAA }\end{array}$ & 40 & $\begin{array}{l}\text { (Christensen et } \\
\text { al. 2014) }\end{array}$ & $\begin{array}{l}\text { For mutant GtoA Rex binding site mutation upstream of sat. Annealed with } \\
\text { DVU1295-RegPLUS-GtoA-R. Forward primer. }\end{array}$ \\
\hline $\begin{array}{l}\text { DVU1295-RegPLUS- } \\
\text { GtoA-R }\end{array}$ & $\begin{array}{l}\text { TTGATTCCGTCTTGTGAAAAAATT } \\
\text { TATAAAATGTCAAGCG } \\
\end{array}$ & 40 & $\begin{array}{l}\text { (Christensen et } \\
\text { al. 2014) }\end{array}$ & $\begin{array}{l}\text { For mutant GtoA Rex binding site mutation upstream of sat. Annealed with } \\
\text { DVU1295-RegPLUS-GtoA-F. Reverse primer. }\end{array}$ \\
\hline $\begin{array}{l}\text { DVU1295-RegPLUS- } \\
\text { IR1-F }\end{array}$ & $\begin{array}{l}\text { CGCTTGACATTTTACGAATT'TTTTC } \\
\text { ACAAGACGGAATCAA }\end{array}$ & 40 & $\begin{array}{l}\text { (Christensen et } \\
\text { al. 2014) }\end{array}$ & $\begin{array}{l}\text { For mutant IR1 Rex binding site mutation upstream of sat. Annealed with } \\
\text { DVU1295-RegPLUS-IR1-R. Forward primer. }\end{array}$ \\
\hline $\begin{array}{l}\text { DVU1295-RegPLUS- } \\
\text { IR1-R }\end{array}$ & $\begin{array}{l}\text { TTGATTCCGTCTTGTGAAAAAATT } \\
\text { CGTAAAATGTCAAGCG }\end{array}$ & 40 & $\begin{array}{l}\text { (Christensen et } \\
\text { al. 2014) }\end{array}$ & $\begin{array}{l}\text { For mutant IR1 Rex binding site mutation upstream of sat. Annealed with } \\
\text { DVU1295-RegPLUS-IR1-F. Reverse primer. }\end{array}$ \\
\hline $\begin{array}{l}\text { DVU1295-RegPLUS- } \\
\text { IR2-F }\end{array}$ & $\begin{array}{l}\text { CGCT'TGACATT'TTGTAAATTTTT'TTT } \\
\text { GTAAGACGGAATCAA }\end{array}$ & 40 & $\begin{array}{l}\text { (Christensen et } \\
\text { al. 2014) }\end{array}$ & $\begin{array}{l}\text { For mutant IR2 Rex binding site mutation upstream of sat. Annealed with } \\
\text { DVU1295-RegPLUS-IR2-R. Forward primer. }\end{array}$ \\
\hline $\begin{array}{l}\text { DVU1295-RegPLUS- } \\
\text { IR2-R }\end{array}$ & $\begin{array}{l}\text { TTGATTCCGTCTTACAAAAAAATT } \\
\text { TACAAAATGTCAAGCG }\end{array}$ & 40 & $\begin{array}{l}\text { (Christensen et } \\
\text { al. 2014) }\end{array}$ & $\begin{array}{l}\text { For mutant IR2 Rex binding site mutation upstream of sat. Annealed with } \\
\text { DVU1295-RegPLUS-IR2-F. Reverse primer. }\end{array}$ \\
\hline $\begin{array}{l}\text { DVU1295-RegPLUS- } \\
\text { IR1and2-F }\end{array}$ & $\begin{array}{l}\text { CGCTTGACATTTTACGAATTT'TTTT'T } \\
\text { GTAAGACGGAATCAA }\end{array}$ & 40 & $\begin{array}{l}\text { (Christensen et } \\
\text { al. 2014) }\end{array}$ & $\begin{array}{l}\text { For mutant IR1and2 Rex binding site mutation upstream of sat. Annealed with } \\
\text { DVU1295-RegPLUS-IR1and2-R. Forward primer. }\end{array}$ \\
\hline $\begin{array}{l}\text { DVU1295-RegPLUS- } \\
\text { IR1and2-R }\end{array}$ & $\begin{array}{l}\text { TTGATTCCGTCTTACAAAAAAATT } \\
\text { CGTAAAATGTCAAGCG }\end{array}$ & 40 & $\begin{array}{l}\text { (Christensen et } \\
\text { al. 2014) }\end{array}$ & $\begin{array}{l}\text { For mutant IR1and2 Rex binding site mutation upstream of sat. Annealed with } \\
\text { DVU1295-RegPLUS-IR1and2-F. Reverse primer. }\end{array}$ \\
\hline
\end{tabular}




\section{Section 7. 7. 2. Fluorescent Polarization Assay (FPA)}

Fluorescent polarization was performed as described (Novichkov et al. 2014) by Dr. Dmitry Rodionov. Fluorescently labeled DNA was designed (Table 7-7) and supplied along with $\operatorname{Rex}_{\mathrm{DvH}}$ to his laboratory for his contribution to this work. The binding assay was performed in a 96-well black plate (VWR, Radnor PA) with $1 \mathrm{nM}$ fluorescently labeled (6-FAM ${ }^{\mathrm{TM}}$ ) oligonucleotides. Different concentrations of protein (10, 25, 50, 100, 250, 500 and 1000 $\mathrm{nM}$ ) were incubated with $1 \mathrm{nM}$ labeled oligonucleotides in $100-\mu$ l reaction mixture in the binding buffer: $20 \mathrm{mM}$ Tris- $\mathrm{HCl}, \mathrm{pH} 7.5 ; 100 \mathrm{mM} \mathrm{NaCl} ; 0.3 \mathrm{mg} / \mathrm{ml}$ of BSA; and $1 \mu \mathrm{g}$ of herring sperm DNA. The fluorescence reading was taken on a Beckman multimode plate reader (DTX 880) with excitation and emission filters at 495 and $520 \mathrm{~nm}$. The background fluorescence from buffer was subtracted and the Fluorescence Polarization values were defined as follows.

$$
\mathrm{P}_{(\mathrm{mp})=}{\underline{\mathrm{I}_{(\text {parallel })}-\left((\mathrm{G} \text {-factor }) * \mathrm{I}_{(\text {perpendicular })}\right)}}^{\mathrm{I}_{(\text {parallel })}+\left((\mathrm{G} \text {-factor }) * \mathrm{I}_{(\text {perpendicular })}\right)} * 1000
$$

I (parallel) and I (perpendicular) are the fluorescence intensity in the parallel and perpendicular orientation respective to the orientation of the excitation polarizer. The Gfactor is an experimental correction for the polarization bias of the detection system (Titolo et al. 2003). 
Table 7-7: Primer List for Fluorescent Polarization Assay (FPA)

\begin{tabular}{|c|c|c|c|c|}
\hline Name & Sequence $\left(5^{\prime}-3^{\prime}\right)$ & $\mathrm{bp}$ & Source & Descriptor \\
\hline DVU0694-3FAM-F & $\begin{array}{l}\text { gggggTTCGTGAAATATTTCAC } \\
\text { CTTggggt }\end{array}$ & 30 & $\begin{array}{l}\text { (Christensen et } \\
\text { al. 2014) }\end{array}$ & $\begin{array}{l}\text { Rex binding site upstream of DVU0694 (qrcB). Annealed } \\
\text { with DVU0694-R. Contains 3-FAM. Forward Primer. }\end{array}$ \\
\hline DVU0694-R & $\begin{array}{l}\text { accccAAGGTGAAATATTTCAC } \\
\text { GAAccccc }\end{array}$ & 30 & $\begin{array}{l}\text { (Christensen et } \\
\text { al. 2014) }\end{array}$ & $\begin{array}{l}\text { Rex binding site upstream of DVU0694 ( } q r c B) \text {. Annealed } \\
\text { with DVU0694-3FAM-F. Reverse Primer. }\end{array}$ \\
\hline DVU0780-3FAM-F & $\begin{array}{l}\text { gggggT'TTGAGCTTTAATTCAC } \\
\text { AACggggt }\end{array}$ & 30 & $\begin{array}{l}\text { (Christensen et } \\
\text { al. 2014) }\end{array}$ & $\begin{array}{l}\text { Rex binding site upstream of DVU0780 (atpF1). Annealed } \\
\text { with DVU0780-R. Contains 3-FAM. Forward Primer. }\end{array}$ \\
\hline DVU0780-R & $\begin{array}{l}\text { accccGTTGTGAATTAAAGCTC } \\
\text { AAAссссс }\end{array}$ & 30 & $\begin{array}{l}\text { (Christensen et } \\
\text { al. 2014) }\end{array}$ & $\begin{array}{l}\text { Rex binding site upstream of DVU00780 (atpF1). Annealed } \\
\text { with DVU0780-3FAM-F. Reverse Primer. }\end{array}$ \\
\hline DVU0402-3FAM-F & $\begin{array}{l}\text { gggggTTTGTCCAAAAAATCAC } \\
\text { GAGggggt }\end{array}$ & 30 & $\begin{array}{l}\text { (Christensen et } \\
\text { al. 2014) }\end{array}$ & $\begin{array}{l}\text { Rex binding site upstream of DVU0402 }(d s r A) \text {. Annealed } \\
\text { with DVU0402-R. Contains 3-FAM. Forward Primer. }\end{array}$ \\
\hline DVU0402-R & $\begin{array}{l}\text { accccCTCGTGATTTTTTGGAC } \\
\text { AAAссссс }\end{array}$ & 30 & $\begin{array}{l}\text { (Christensen et } \\
\text { al. 2014) }\end{array}$ & $\begin{array}{l}\text { Rex binding site upstream of DVU0402 }(d s r A) \text {. Annealed } \\
\text { with DVU0402-3FAM-F. Reverse Primer. }\end{array}$ \\
\hline DVU1636-3FAM-F & $\begin{array}{l}\text { gggggATTGTGCTATTTGGCAC } \\
\text { AAAggggt }\end{array}$ & 30 & $\begin{array}{l}\text { (Christensen et } \\
\text { al. 2014) }\end{array}$ & $\begin{array}{l}\text { Rex binding site upstream of DVU1636 }(p p a C) \text {. Annealed } \\
\text { with DVU1636-R. Contains 3-FAM. Forward Primer. }\end{array}$ \\
\hline DVU1636-R & $\begin{array}{c}\text { accccTTTGTGCCAAATAGCAC } \\
\text { AATссссс }\end{array}$ & 30 & $\begin{array}{l}\text { (Christensen et } \\
\text { al. 2014) }\end{array}$ & $\begin{array}{l}\text { Rex binding site upstream of DVU1636 }(p p a C) \text {. Annealed } \\
\text { with DVU1636-3FAM-F. Reverse Primer. }\end{array}$ \\
\hline DVU1917-3FAM-F & $\begin{array}{l}\text { gggggCGAGCTATATATTTCAC } \\
\text { AAAggggt }\end{array}$ & 30 & $\begin{array}{l}\text { (Christensen et } \\
\text { al. 2014) }\end{array}$ & $\begin{array}{l}\text { Rex binding site upstream of DVU1917 (bysB). Annealed } \\
\text { with DVU1917-R. Contains 3-FAM. Forward Primer. }\end{array}$ \\
\hline DVU1917-R & $\begin{array}{c}\text { accccTTTGTGAAATATATAGC } \\
\text { TCGсcсcс }\end{array}$ & 30 & $\begin{array}{l}\text { (Christensen et } \\
\text { al. 2014) }\end{array}$ & $\begin{array}{l}\text { Rex binding site upstream of DVU1917 (bysB). Annealed } \\
\text { with DVU1917-3FAM-F. Reverse Primer. }\end{array}$ \\
\hline DVU1295-3FAM-F & $\begin{array}{c}\text { gggggTTTGTAAATTTTTTCAC } \\
\text { AAGggggt }\end{array}$ & 30 & $\begin{array}{l}\text { (Christensen et } \\
\text { al. 2014) }\end{array}$ & $\begin{array}{l}\text { Rex binding site upstream of DVU1295 (sat). Annealed } \\
\text { with DVU1295-R. Contains 3-FAM. Forward Primer. }\end{array}$ \\
\hline DVU1295-R & $\begin{array}{c}\text { accccCTTGTGAAAAAATTTAC } \\
\text { AAAсcссс }\end{array}$ & 30 & $\begin{array}{l}\text { (Christensen et } \\
\text { al. 2014) }\end{array}$ & $\begin{array}{l}\text { Rex binding site upstream of DVU1295 (sat). Annealed } \\
\text { with DVU1295-3FAM-F. Reverse Primer. }\end{array}$ \\
\hline DVU0920-3FAM-F & $\begin{array}{c}\text { gggggCTTGTGAACGATTGCAC } \\
\text { GAAggggt }\end{array}$ & 30 & $\begin{array}{l}\text { (Christensen et } \\
\text { al. 2014) }\end{array}$ & $\begin{array}{l}\text { Rex binding site upstream of DVU0920 (atpI). Annealed } \\
\text { with DVU0920-R. Contains 3-FAM. Forward Primer. }\end{array}$ \\
\hline DVU0920-R & $\begin{array}{c}\text { accccTTCGTGCAATCGTTCAC } \\
\text { AAGccсcс }\end{array}$ & 30 & $\begin{array}{l}\text { (Christensen et } \\
\text { al. 2014) }\end{array}$ & $\begin{array}{l}\text { Rex binding site upstream of DVU0920 (atpI). Annealed } \\
\text { with DVU0920-3FAM-F. Reverse Primer. }\end{array}$ \\
\hline DVU0846-3FAM-F & $\begin{array}{c}\text { gggggATTGTTAATTCCATCAC } \\
\text { AAGggggt }\end{array}$ & 30 & $\begin{array}{l}\text { (Christensen et } \\
\text { al. 2014) }\end{array}$ & $\begin{array}{l}\text { Rex binding site upstream of DVU0846 (apsB). Annealed } \\
\text { with DVU0846-R. Contains 3-FAM. Forward Primer. }\end{array}$ \\
\hline
\end{tabular}




\begin{tabular}{|c|c|c|c|c|}
\hline Name & Sequence $\left(5^{\prime}-3^{\prime}\right)$ & bp & Source & Descriptor \\
\hline DVU0846-R & $\begin{array}{l}\text { accccCTTGTGATGGAATTAAC } \\
\text { AATссссс }\end{array}$ & 30 & $\begin{array}{l}\text { (Christensen et } \\
\quad \text { al. 2014) }\end{array}$ & $\begin{array}{l}\text { Rex binding site upstream of DVU0846 (apsB). Annealed } \\
\text { with DVU0846-3FAM-F. Reverse Primer. }\end{array}$ \\
\hline DVU2791-3FAM-F & $\begin{array}{c}\text { gggggCTTGTGAAATAATGTTC } \\
\text { TTT'Tggggt }\end{array}$ & 30 & $\begin{array}{l}\text { (Christensen et } \\
\quad \text { al. 2014) }\end{array}$ & $\begin{array}{l}\text { Rex binding site upstream of DVU2791 }(d b c A) \text {. Annealed } \\
\text { with DVU2791-R. Contains 3-FAM. Forward Primer. }\end{array}$ \\
\hline DVU2791-R & $\begin{array}{l}\text { accccAAAGAACATTATTTCAC } \\
\text { AAGccсcс }\end{array}$ & 30 & $\begin{array}{l}\text { (Christensen et } \\
\text { al. 2014) }\end{array}$ & $\begin{array}{l}\text { Rex binding site upstream of DVU2791 }(d b c A) \text {. Annealed } \\
\text { with DVU2791-3FAM-F. Reverse Primer. }\end{array}$ \\
\hline DVU1932-3FAM-F & $\begin{array}{c}\text { gggggCTCGTGAAATTAATGAC } \\
\text { AAGggggt }\end{array}$ & 30 & $\begin{array}{l}\text { (Christensen et } \\
\text { al. 2014) }\end{array}$ & $\begin{array}{l}\text { Rex binding site upstream of DVU1932 (adk). Annealed } \\
\text { with DVU1932-R. Contains 3-FAM. Forward Primer. }\end{array}$ \\
\hline DVU1932-R & $\begin{array}{l}\text { accccCTTGTCATTAATTTCAC } \\
\text { GAGccccc }\end{array}$ & 30 & $\begin{array}{l}\text { (Christensen et } \\
\text { al. 2014) }\end{array}$ & $\begin{array}{l}\text { Rex binding site upstream of DVU1932 (adk). Annealed } \\
\text { with DVU1932-3FAM-F. Reverse Primer. }\end{array}$ \\
\hline DVU2286-3FAM-F & $\begin{array}{l}\text { gggggATTGGGAATCGATTCAC } \\
\text { AAAggggt }\end{array}$ & 30 & $\begin{array}{l}\text { (Christensen et } \\
\text { al. 2014) }\end{array}$ & $\begin{array}{l}\text { Rex binding site upstream of DVU2286 (cooM). Annealed } \\
\text { with DVU2286-R. Contains 3-FAM. Forward Primer. }\end{array}$ \\
\hline DVU2286-R & $\begin{array}{l}\text { accccTTTGTGAATCGATTCCC } \\
\text { AATсcссс } \\
\end{array}$ & 30 & $\begin{array}{c}\text { (Christensen et } \\
\text { al. 2014) }\end{array}$ & $\begin{array}{l}\text { Rex binding site upstream of DVU2286 }(c o o M) \text {. Annealed } \\
\text { with DVU2286-3FAM-F. Reverse Primer. }\end{array}$ \\
\hline DVU1292-3FAM-F & $\begin{array}{l}\text { gggggTATGTGAAAAAAATCAT } \\
\text { TTTggggt }\end{array}$ & 30 & $\begin{array}{l}\text { (Christensen et } \\
\text { al. 2014) }\end{array}$ & $\begin{array}{l}\text { Rex binding site upstream of DVU1292 (hypothetical). } \\
\text { Annealed with DVU1292-R. Contains 3-FAM. Forward. }\end{array}$ \\
\hline DVU1292-R & $\begin{array}{l}\text { accccAAAATGATT'TT'T'TCAC } \\
\text { ATAсcсcс }\end{array}$ & 30 & $\begin{array}{l}\text { (Christensen et } \\
\text { al. 2014) }\end{array}$ & $\begin{array}{l}\text { Rex binding site upstream of DVU1292 (hypothetical). } \\
\text { Annealed with DVU1292-3FAM-F. Reverse Primer. }\end{array}$ \\
\hline FDde2476_L & $\begin{array}{l}\text { cccctcagtTTATCAACTTAGT'TT } \\
\text { GATATagtgaccccct }\end{array}$ & 41 & $\begin{array}{l}\text { (Novichkov } \\
\text { et al. 2014) }\end{array}$ & $\begin{array}{l}\text { Negative control. Random AT-rich sequence. Annealed } \\
\text { with GC_FDde2476_L Contains 3-FAM. Forward } \\
\text { Primer. }\end{array}$ \\
\hline GC_FDde2476_L & $\begin{array}{c}\text { agggggtcactATATCAAACTAAGT } \\
\text { TGATAAactgaggggg } \\
\end{array}$ & 41 & $\begin{array}{l}\text { (Novichkov } \\
\text { et al. 2014) }\end{array}$ & $\begin{array}{l}\text { Negative control. Random AT-rich sequence. Annealed } \\
\text { with FDde2476_L. Reverse Primer. }\end{array}$ \\
\hline
\end{tabular}




\section{Section 7. 8. Select protein abundance determination}

Samples for protein analysis by targeted proteomics (Redding-Johanson et al. 2011) were grown and collected as described for the qRT-PCR analysis. Instead of 5-ml cultures, these samples were $100-\mathrm{ml}$ cultures grown in 250-ml bottles. Preliminary transcript analysis between cultures grown in the two separate containers (tube vs bottle) had similar expression (data not shown). For each sample, $40 \mathrm{ml}$ of the respiring cultures (OD600 $=$ $0.250)$ or $80 \mathrm{ml}$ of the pyruvate-fermenting cultures (OD600 $=0.125)$ were pelleted by centrifugation $\left(\sim 3700 \times \mathrm{g} 4^{\circ} \mathrm{C} 12 \mathrm{~min}\right)$, and the supernatant was decanted. Cells were flash frozen in liquid nitrogen and stored at $-80^{\circ} \mathrm{C}$ until use. Protein extracts were then digested with trypsin $(1 \mu \mathrm{g} / \mu \mathrm{l})$ at $37^{\circ} \mathrm{C}$ overnight. Each sample was analyzed on an Agilent Technologies (Santa Clara, CA) 6460QQQ mass spectrometer operating in multiple-reaction monitoring (MRM) mode coupled to an Agilent 1100 system. Five micrograms of peptides were injected onto a Sigma Ascentis Peptide Express C-18 column (2.1 mm x $50 \mathrm{~mm})$ via an Agilent G1377A autosampler. A 23-min method with a 10-min gradient was used and consisted of a $400 \mu \mathrm{l} / \mathrm{min}$ flow rate, starting with $95 \%$ (all \% in this section are vol/vol) Buffer A (98\% water, $2 \%$ acetonitrile, $0.1 \%$ formic acid) and $5 \%$ Buffer B (98 \% acetonitrile, $2 \%$ water, $0.1 \%$ formic acid) for one min, followed by an increase to $40 \%$ Buffer B over $10 \mathrm{~min}$, followed by a rapid increase to $90 \% \mathrm{~B}$ in $5 \mathrm{~min}$, where it was held for 4 min. The solvent composition was quickly ramped to $5 \% \mathrm{~B}$, where it was subsequently held for 2 min to allow the column to equilibrate for the next run. The peptides eluting from the column were ionized by an Agilent Jet Stream source (Sheath Gas flow: 11 1/min nitrogen, Sheath Gas Temperature: 350 C, Nozzle Voltage: 1000 V, Nebulizing Pressure: 30 psi, Chamber Voltage: 4500 V) operating in positive-ion mode. All selected-reaction monitoring (SRM) methods were designed, analyzed, and processed with Skyline software 
version 1.4 (MacLean et al. 2010). Two or three peptides for each protein were selected and validated from a reference $\mathrm{DvH}$ protein digest for quantification by SRM mass spectrometry. The top 2-4 ionizing tryptic peptides $(2+$ or $3+$ charge states) of 8-20 amino acids in length were selected with the default Agilent 6460 collision energy equations for each target protein. The most intense two or three y-series fragment ions for each peptide were monitored and the sum of their integrated peak areas was given for peptide quantification. Peptide areas for multiple peptides of the same protein were summed to assign relative area abundance to that protein. This information along with the experimentally determined retention times were used to build a scheduled MRM method with a retention time window of 3 min to optimize speed and sensitivity. The error bars represent the standard deviation of three biological replicates. The data were plotted with Spotfire 3.2.1 (TIBCO Software, http://spotfire.tibco.com), a data analysis and visualization package.

\section{Section 7.9. Transcription start site (TSS) of sat determined by $5^{\prime}-\mathrm{RACE}$}

Samples were initially collected from DNase-treated RNA samples. The procedure used was adapted from Scotto-Lavino et al. (Scotto-Lavino et al. 2006). A schematic is shown in Figure 7-6 and primers in Table 7-8. In brief, 100 ng of DNase treated RNA was reverse transcribed to single-stranded cDNA (iScript ${ }^{\mathrm{TM}}$ Select cDNA Synthesis Kit, Bio-Rad) with primer DVU1295-sat-GSP1, degraded with RNase A/T1 Mix and RNaseH (Thermo Fisher Scientific) and the cDNA was purified by column purification. With terminal deoxynucleotidyl transferase (Thermo Fisher Scientific), an adenosine tail was added to the cDNA, followed by generation of the second DNA strand with iScript ${ }^{\mathrm{TM}}$ and primer RACE- 
$2^{\text {nd }}$ Strand. The RACE- ${ }^{\text {nd }}$ Strand primer was adapted from primer AUAP, Invitrogen 5'

RACE System for Rapid Amplification of cDNA Ends Version 2.0, to include additional T residues and a "V" at the 3' position ("V" as A/G/C, but not T) to allow for better anchoring to the modified cDNA. dsDNA was purified, diluted 1:1000 and amplified by PCR to generate DNA fragment PCR1, with primers: DVU1295-GSP2 and AUAP. Thirty cycles of amplification were carried out $\left(30 \mathrm{sec}\right.$ at $94^{\circ} \mathrm{C}, 30 \mathrm{sec}$ at $58^{\circ} \mathrm{C}, 1 \mathrm{~min}$ at $\left.72^{\circ} \mathrm{C}\right)$ followed by a final extension for $5 \mathrm{~min}$ at $72^{\circ} \mathrm{C}$ with Taq DNA Polymerase (NEB). An additional PCR (PCR2) followed, which was the same protocol as PCR1, but with 1:1000 diluted PCR1 as template and nested primers (AUAP and DVU1295-GSP3). Fragments were purified, sequenced, and mapped to the genome. Additional discussion of the complexities of the 5'-RACE experiment is described in Chapter 5.

Table 7-8: Primer List for 5'-Rapid Amplification of cDNA Ends (5'-RACE)

\begin{tabular}{|c|c|c|c|c|}
\hline Name & Sequence (5'-3’) & bp & Source & Descriptor \\
\hline DVU1295-sat-GSP1 & $\begin{array}{l}\text { GACGAGAGCGATT'T } \\
\text { CC }\end{array}$ & 16 & $\begin{array}{l}\text { (Christensen } \\
\text { et al. 2014) }\end{array}$ & $\begin{array}{l}\text { Gene specific primer (GSP1) for } \\
\text { cDNA generation of sat } \\
\text { transcript. Reverse Primer. }\end{array}$ \\
\hline RACE-2nd Strand & $\frac{\frac{\text { GGCCACGCGTCGAC }}{\text { TAGTACTTTTTT'TTT }}}{\text { TTTTTTTTTTV }}$ & 39 & $\begin{array}{l}\text { (Christensen } \\
\text { et al. 2014) }\end{array}$ & $\begin{array}{l}\text { For generation of complementary } \\
\text { strand of sat cDNA. Underlined } \\
\text { portion contains overhang } \\
\text { region for amplification by } \\
\text { PCR. "V" denotes base A/G/C } \\
\text { but not T. }\end{array}$ \\
\hline AUAP & $\begin{array}{l}\text { GGCCACGCGTCGAC } \\
\text { TAGTAC }\end{array}$ & 20 & $\begin{array}{l}\text { Invitrogen } 5^{\prime}- \\
\text { RACE system }\end{array}$ & $\begin{array}{l}\text { Abridged Universal Amplification } \\
\text { Primer. For amplification of sat } \\
\text { cDNA for PCR1 (with primer } \\
\text { DVU1295-sat-GSP2) or PCR2 } \\
\text { (with primer DVU1295-sat- } \\
\text { GSP3). }\end{array}$ \\
\hline DVU1295-sat-GSP2 & $\begin{array}{l}\text { CGTCGTCCTTGGAC } \\
\text { ACGTCA }\end{array}$ & 20 & $\begin{array}{l}\text { (Christensen } \\
\text { et al. 2014) }\end{array}$ & $\begin{array}{l}\text { (GSP2) For amplification of sat } \\
\text { cDNA with primer AUAP } \\
\text { (PCR1). }\end{array}$ \\
\hline DVU1295-sat-GSP3 & $\begin{array}{l}\text { ATTTCGATCTGCTT' } \\
\text { GAGGCCGG }\end{array}$ & 22 & $\begin{array}{l}\text { (Christensen } \\
\text { et al. 2014) }\end{array}$ & $\begin{array}{l}\text { (GSP3) For nested amplification } \\
\text { of PCR1 fragment with primer } \\
\text { AUAP (PCR2). For sequencing } \\
\text { of PCR2 fragment. }\end{array}$ \\
\hline
\end{tabular}




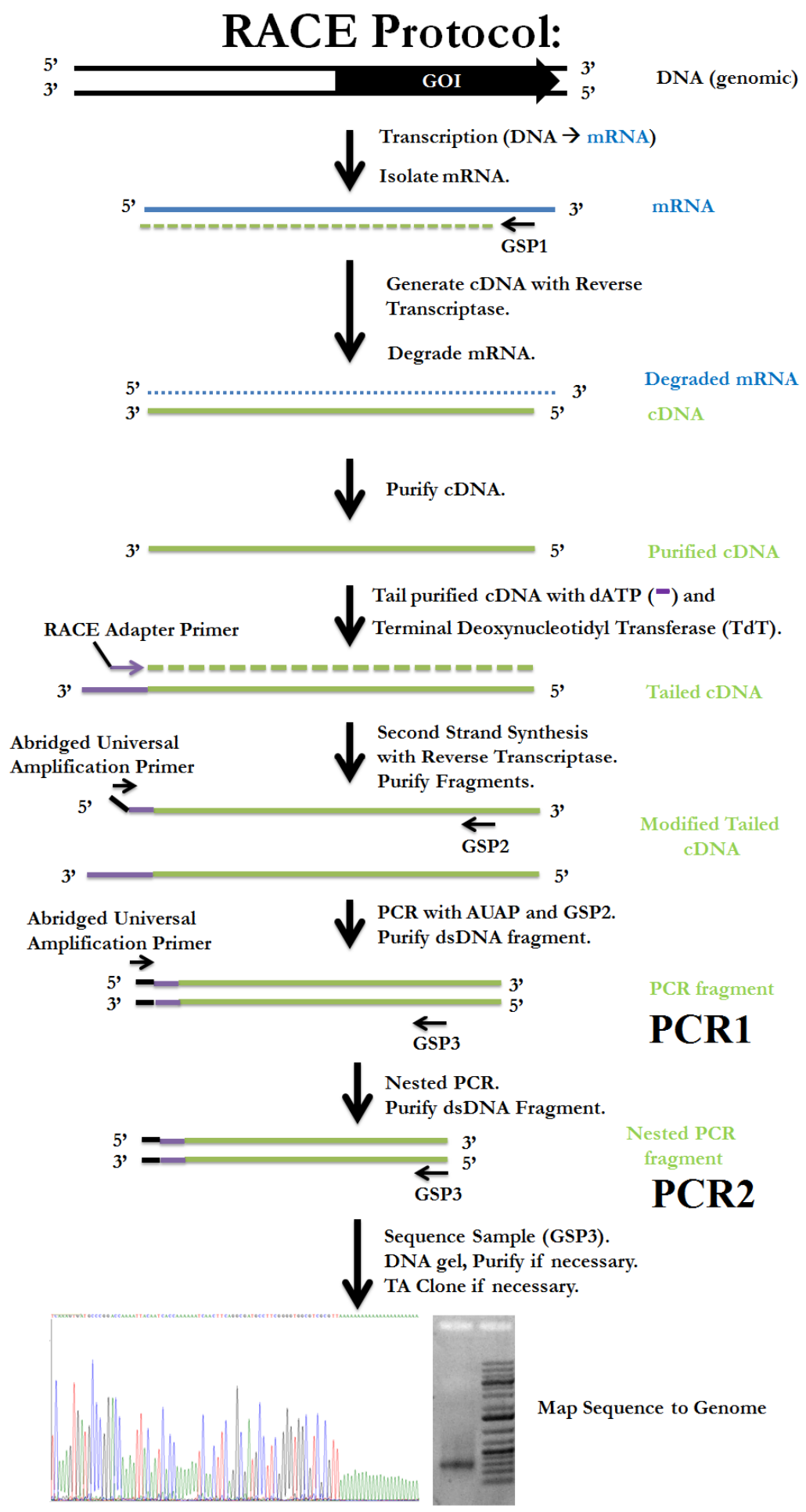

Figure 7-6: Method to determine TSS. Simplified schematic of 5'-Rapid Amplification of cDNA Ends (5'-RACE) for sat. 


\section{Section 7. 10. Whole-genome fitness study by TnLE-seq}

This Rex mutant gene-fitness study presented here for the first time was part of a larger study (Fels et al. 2013; Korte et al. 2014), where five TnLE-seq pools were multiplexed for deep sequencing by the Illumina ${ }^{\mathrm{TM}}$ procedure. Complete data are available at http://desulfovibriomaps.biochem.missouri.edu/fitness/. Gene fitness is shown by $\log _{2} \mathrm{R}$ format, where $\mathrm{R}$ is the ratio of the number of insertions in a gene in the Rex mutant compared to the insertions in that gene in the parental strain. A positive $\mathrm{R}$ correlates to a fitness advantage and a negative $\mathrm{R}$ to a fitness defect. A gene whose fitness was $>-1.7$ was considered non-essential. For those genes with a fitness score between -1.7 and -2.2 , they were classified as "synthetic sick" (Fels et al. 2013). As previously published (Fels et al. 2013), the fitness was only determined for genes with insertions within the 5-85\% region of the coding sequence, as these mutations are most likely to affect the protein function of the gene product. Interestingly, there are sequences for transposon insertions within the rex gene in the strain deleted for rex. This phenomenon was attributed to "barcode bleed," an artifact of assaying multiple pools on the same Illumina ${ }^{\mathrm{TM}}$ HiSeq lane (Kircher et al. 2010).

\section{Section 7.11. $\mathrm{NAD}^{+}$and $\mathrm{NADH}$ determination}

The NADH/NAD ${ }^{+}$quantification kit (Sigma) was used to measure the concentration of pyridine nucleotides. The experiment was followed according to manufacturer's protocol. As a proof of principal experiment, $\alpha$-select silver efficiency E. coli cells were first tested. In brief, $5 \mathrm{ml} \mathrm{LC}$ was inoculated with $50 \mu \mathrm{l}$ cells and incubated $12 \mathrm{hrs}$ while shaking at $37^{\circ} \mathrm{C}$. Cells were washed twice with PBS by centrifugation at $\sim 2,000 \mathrm{xg}$ for $5 \mathrm{~min}$. Cells were extracted in $400 \mu \mathrm{l}$ NADH/NAD Extraction buffer by freeze/thaw methods (20 min dry ice/ $10 \mathrm{~min}$ at room temperature, twice). Samples were then vortexed and centrifuged 
(13,000 x g $10 \mathrm{~min})$ and the supernatant was passed over a PD-10 desalting column (GE Healthcare) and stored on ice. The assay to determine the pyridine concentration is a cycling reaction that converts $\mathrm{NADH}$ to $\mathrm{NAD}^{+}$. Therefore, the supernatant is split into two samples, one to determine $\mathrm{NAD}_{\text {total }}$ and one to determine $\mathrm{NAD}^{+} . \mathrm{NADH}$ is determined by subtracting $\mathrm{NAD}^{+}$from $\mathrm{NAD}_{\text {total }}$ Following the manufacturer's protocol the samples are read (A450) in a 96-well format with a Synergy Mx monochromator-based multi-mode microplate reader (BioTek, Winooski, VT) and the concentration of the pyridine nucleotides are determined by comparing the unknowns to a standard curve. A discussion of the practicality of this experiment is described in Chapter 5. 


\section{References}

Agari, Y., A. Kashihara, S. Yokoyama, S. Kuramitsu and A. Shinkai (2008). "Global gene expression mediated by Thermus thermophilus SdrP, a CRP/FNR family transcriptional regulator." Mol Microbiol 70(1): 60-75.

Aketagawa, J., K. Kobayashi and M. Ishimoto (1985). "Purification and properties of thiosulfate reductase from Desulfovibrio vulgaris, Miyazaki F." J Biochem 97(4): 10251032.

Al-Karadaghi, S., E. S. Cedergren-Zeppezauer and S. Hovmoller (1994). "Refined crystal structure of liver alcohol dehydrogenase-NADH complex at 1.8 A resolution." Acta Crystallogr D Biol Crystallogr 50(Pt 6): 793-807.

Altuvia, S., M. Almiron, G. Huisman, R. Kolter and G. Storz (1994). "The dps promoter is activated by OxyR during growth and by IHF and sigma $\mathrm{S}$ in stationary phase." $\mathrm{Mol}$ Microbiol 13(2): 265-272.

Arnold, K., L. Bordoli, J. Kopp and T. Schwede (2006). "The SWISS-MODEL workspace: a web-based environment for protein structure homology modelling." Bioinformatics 22(2): 195-201.

Badziong, W. and R. K. Thauer (1978). "Growth yields and growth rates of Desulfovibrio vulgaris (Marburg) growing on hydrogen plus sulfate and hydrogen plus thiosulfate as the sole energy sources." Archives of Microbiology 117(2): 209-214.

Baena, S., M. L. Fardeau, M. Labat, B. Ollivier, J. L. Garcia and B. K. Patel (1998). "Desulfovibrio aminophilus sp. nov., a novel amino acid degrading and sulfate reducing bacterium from an anaerobic dairy wastewater lagoon." Systematic and Applied Microbiology 21(4): 498-504.

Barton, L. (1995). Sulfate-reducing bacteria. New York, Plenum Press.

Barton, L. and W. A. Hamilton (2007). Sulphate-reducing bacteria: environmental and engineered systems. Cambridge ; New York, Cambridge University Press.

Bauer, C. E., S. Elsen and T. H. Bird (1999). "Mechanisms for redox control of gene expression." Annual Review of Microbiology 53: 495-523.

Beech, I. B. and J. A. Sunner (2007). "Sulphate-reducing bacteria and their role in corrosion of ferrous materials." Sulphate-Reducing Bacteria: Environmental and Engineered Systems: $459-482$.

Beijerinck, M. W. (1895). Zentbl. Bakt. Parasit Kde abt. 2(1): 1-9, 49-59, 104-114.

Biasini, M., S. Bienert, A. Waterhouse, K. Arnold, G. Studer, T. Schmidt, F. Kiefer, T. G. Cassarino, M. Bertoni, L. Bordoli and T. Schwede (2014). "SWISS-MODEL: 
modelling protein tertiary and quaternary structure using evolutionary information." Nucleic Acids Res 42(Web Server issue): W252-258.

Biswas, K. C., N. A. Woodards, H. Xu and L. L. Barton (2009). "Reduction of molybdate by sulfate-reducing bacteria." Biometals 22(1): 131-139.

Bitoun, J. P., S. Liao, X. Yao, G. G. Xie and Z. T. Wen (2012). "The redox-sensing regulator Rex modulates central carbon metabolism, stress tolerance response and biofilm formation by Streptococcus mutans." PLoS One 7(9): e44766.

Bitoun, J. P., A. H. Nguyen, Y. Fan, R. A. Burne and Z. T. Wen (2011). "Transcriptional repressor Rex is involved in regulation of oxidative stress response and biofilm formation by Streptococcus mutans." FEMS Microbiol Lett 320(2): 110-117.

Bradford, M. M. (1976). "A rapid and sensitive method for the quantitation of microgram quantities of protein utilizing the principle of protein-dye binding." Anal Biochem 72: $248-254$.

Brekasis, D. and M. S. Paget (2003). "A novel sensor of NADH/NAD ${ }^{+}$redox poise in Streptomyces coelicolor A3(2)." Embo Journal 22(18): 4856-4865.

Broco, M., M. Rousset, S. Oliveira and C. Rodrigues-Pousada (2005). "Deletion of flavoredoxin gene in Desulfovibrio gigas reveals its participation in thiosulfate reduction." FEBS Lett 579(21): 4803-4807.

Brown, J. V. Stoyanov, S. P. Kidd and J. L. Hobman (2003). "The MerR family of transcriptional regulators." Fems Microbiology Reviews 27(2-3): 145-163.

Brown, T. (1993). Analysis of DNA sequences by blotting and hybridization. Current protocols in molecular biology. F. M. Ausubel, R. Brent, R. E. Kingstonet al. New York, NY, John Wiley and Sons: 2.9.1-2.9.20.

Burgess, R. R., A. A. Travers, J. J. Dunn and E. K. Bautz (1969). "Factor stimulating transcription by RNA polymerase." Nature 221(5175): 43-46.

Busby, S. and R. H. Ebright (1999). "Transcription activation by catabolite activator protein (CAP)." L Mol Biol 293(2): 199-213.

Bushman, J. B. (2001). Financial Impact of Corrosion on the Economy of the United States. Ohio, USA, Bushman \& Associates, Inc.

Chadsey, M. S., J. E. Karlinsey and K. T. Hughes (1998). "The flagellar anti-sigma factor FlgM actively dissociates Salmonella typhimurium sigma ${ }^{28}$ RNA polymerase holoenzyme." Genes Dev 12(19): 3123-3136.

Chomczynski, P. and N. Sacchi (1987). "Single-step method of RNA isolation by acid guanidinium thiocyanate-phenol-chloroform extraction." Anal Biochem 162(1): 156159. 
Chomczynski, P. and N. Sacchi (2006). "The single-step method of RNA isolation by acid guanidinium thiocyanate-phenol-chloroform extraction: twenty-something years on." Nat Protoc 1(2): 581-585.

Christensen, G. A., G. M. Zane, A. E. Kazakov, Li X, D. A. Rodionov, P. Novichkov, I. Dubchak, A. P. Arkin and J. D. Wall (2015). "Rex (encoded by DVU0916) in Desulfovibrio vulgaris Hildenborough is a repressor of sulfate adenylyl transferase and is regulated by NADH." J. Bacteriol. 197(1):29-39.

Cypionka, H. (2000). "Oxygen respiration by Desulfovibrio species." Annual Review of Microbiology 54: 827-848.

Dahl, C. and C. G. Friedrich (2008). Microbial sulfur metabolism. Berlin ; New York, Springer.

Davis, J. R. (2000). Corrosion : understanding the basics. Materials Park, Ohio, ASM International.

Dehal, P. S., M. P. Joachimiak, M. N. Price, J. T. Bates, J. K. Baumohl, D. Chivian, G. D. Friedland, K. H. Huang, K. Keller, P. S. Novichkov, I. L. Dubchak, E. J. Alm and A. P. Arkin (2010). "MicrobesOnline: an integrated portal for comparative and functional genomics." Nucleic Acids Res 38: D396-D400.

Don, R. H., P. T. Cox, B. J. Wainwright, K. Baker and J. S. Mattick (1991). "'Touchdown' PCR to circumvent spurious priming during gene amplification." Nucleic Acids Res 19(14): 4008.

Du, X. and J. J. Pene (1999). "Identification, cloning and expression of p25, an AT-rich DNA-binding protein from the extreme thermophile, Thermus aquaticus YT-1." Nucleic Acids Res 27(7): 1690-1697.

Ebright, R. H. and S. Busby (1995). "The Escherichia coli RNA polymerase alpha subunit: structure and function." Curr Opin Genet Dev 5(2): 197-203.

Enning, D. and J. Garrelfs (2013). "Corrosion of iron by sulfate-reducing bacteria - new views of an old problem." Appl Environ Microbiol.

Fels, S. R., G. M. Zane, S. M. Blake and J. D. Wall (2013). "Rapid transposon liquid enrichment sequencing (TnLE-seq) for gene fitness evaluation in underdeveloped bacterial systems." Appl Environ Microbiol 79(23): 7510-7517.

Findley, J. E. and J. M. Akagi (1970). "Role of thiosulfate in bisulfite reduction as catalyzed by Desulfovibrio vulgaris." L Bacteriol 103(3): 741-744.

Finnin, M. S., J. R. Donigian and N. P. Pavletich (2001). "Structure of the histone deacetylase SIRT2." Nat Struct Biol 8(7): 621-625. 
Finster, K., W. Liesack and B. Thamdrup (1998). "Elemental sulfur and thiosulfate disproportionation by Desulfocapsa sulfoexigens sp. nov., a new anaerobic bacterium isolated from marine surface sediment." Appl Environ Microbiol 64(1): 119-125.

Fritz, G., A. Roth, A. Schiffer, T. Buchert, G. Bourenkov, H. D. Bartunik, H. Huber, K. O. Stetter, P. M. Kroneck and U. Ermler (2002). "Structure of adenylylsulfate reductase from the hyperthermophilic Archaeoglobus fulgidus at 1.6-A resolution." Proc Natl Acad Sci U S A 99(4): 1836-1841.

Fu, R., J. D. Wall and G. Voordouw (1994). "DcrA, a c-type heme-containing methylaccepting protein from Desulfovibrio vulgaris Hildenborough, senses the oxygen concentration or redox potential of the environment." L Bacteriol 176(2): 344-350.

Goujon, M., H. McWilliam, W. Li, F. Valentin, S. Squizzato, J. Paern and R. Lopez (2010). "A new bioinformatics analysis tools framework at EMBL-EBI." Nucleic Acids Res 38(Web Server issue): W695-699.

Goulhen, F., A. Gloter, F. Guyot and M. Bruschi (2006). "Cr(VI) detoxification by Desulfovibrio vulgaris strain Hildenborough: microbe-metal interactions studies." Appl Microbiol Biotechnol 71(6): 892-897.

Gourse, R. L., W. Ross and T. Gaal (2000). "UPs and downs in bacterial transcription initiation: the role of the alpha subunit of RNA polymerase in promoter recognition." Mol Microbiol 37(4): 687-695.

Greenberg, J. T., P. Monach, J. H. Chou, P. D. Josephy and B. Demple (1990). "Positive control of a global antioxidant defense regulon activated by superoxide-generating agents in Escherichia coli." Proc Natl Acad Sci U S A 87(16): 6181-6185.

Greenwald, J., C. Buhtz, C. Ritter, W. Kwiatkowski, S. Choe, M. L. Maddelein, F. Ness, S. Cescau, A. Soragni, D. Leitz, S. J. Saupe and R. Riek (2010). "The mechanism of prion inhibition by HET-S." Mol Cell 38(6): 889-899.

Grigorova, I. L., N. J. Phleger, V. K. Mutalik and C. A. Gross (2006). "Insights into transcriptional regulation and sigma competition from an equilibrium model of RNA polymerase binding to DNA." Proc Natl Acad Sci U S A 103(14): 5332-5337.

Gruber, T. M. and C. A. Gross (2003). "Multiple sigma subunits and the partitioning of bacterial transcription space." Annual Review of Microbiology 57: 441-466.

Guex, N., M. C. Peitsch and T. Schwede (2009). "Automated comparative protein structure modeling with SWISS-MODEL and Swiss-PdbViewer: a historical perspective." Electrophoresis 30 Suppl 1: S162-173.

Gyan, S., Y. Shiohira, I. Sato, M. Takeuchi and T. Sato (2006). "Regulatory loop between redox sensing of the $\mathrm{NADH} / \mathrm{NAD}(+)$ ratio by $\operatorname{Rex}(\mathrm{YdiH})$ and oxidation of NADH by NADH dehydrogenase Ndh in Bacillus subtilis." L Bacteriol 188(20): 7062-7071. 
Hall, T. A. (1999). BioEdit: a user-friendly biological sequence alignment editor and analysis program for Windows 95/98/NT. Nucleic Acids Symposium Series: 95-98.

Hamilton, W. A. (2003). "Microbially influenced corrosion as a model system for the study of metal microbe interactions: A unifying electron transfer hypothesis." Biofouling 19(1): 65-76.

Haschke, R. H. and L. L. Campbell (1971). "Thiosulfate reductase of Desulfovibrio vulgaris." I Bacteriol 106(2): 603-607.

Hays, G. H. (2010). "Now is the Time." Fall.

Heidelberg, J. F., R. Seshadri, S. A. Haveman, C. L. Hemme, I. T. Paulsen, J. F. Kolonay, J. A. Eisen, N. Ward, B. Methe, L. M. Brinkac, S. C. Daugherty, R. T. Deboy, R. J. Dodson, A. S. Durkin, R. Madupu, W. C. Nelson, S. A. Sullivan, D. Fouts, D. H. Haft, J. Selengut, J. D. Peterson, T. M. Davidsen, N. Zafar, L. W. Zhou, D. Radune, G. Dimitrov, M. Hance, K. Tran, H. Khouri, J. Gill, T. R. Utterback, T. V. Feldblyum, J. D. Wall, G. Voordouw and C. M. Fraser (2004). "The genome sequence of the anaerobic, sulfate-reducing bacterium Desulfovibrio vulgaris Hildenborough." Nat Biotechnol 22(5): 554-559.

Heldwein, E. E. and R. G. Brennan (2001). "Crystal structure of the transcription activator BmrR bound to DNA and a drug." Nature 409(6818): 378-382.

Hellemans, J., G. Mortier, A. De Paepe, F. Speleman and J. Vandesompele (2007). "qBase relative quantification framework and software for management and automated analysis of real-time quantitative PCR data." Genome Biology 8(2): R19.

Hocking, W. P., R. Stokke, I. Roalkvam and I. H. Steen (2014). "Identification of key components in the energy metabolism of the hyperthermophilic sulfate-reducing archaeon Archaeoglobus fulgidus by transcriptome analyses." Front Microbiol 5: 95.

Huang, X. Q. and W. Miller (1991). "A Time-Efficient, Linear-Space Local Similarity Algorithm." Advances in Applied Mathematics 12(3): 337-357.

Humphrey, W., A. Dalke and K. Schulten (1996). "VMD: Visual molecular dynamics." Journal of Molecular Graphics \& Modelling 14(1): 33-38.

Hurt, R. A., Jr., S. D. Brown, M. Podar, A. V. Palumbo and D. A. Elias (2012). "Sequencing intractable DNA to close microbial genomes." PLoS One 7(7): e41295.

Iuchi, S. and E. C. Lin (1988). "arcA (dye), a global regulatory gene in Escherichia coli mediating repression of enzymes in aerobic pathways." Proc Natl Acad Sci U S A 85(6): 1888-1892.

Jorgensen, B. B. (1977). "The sulfur cycle of a coastal marine sediment." Limnology and Oceanography 22: 814-832. 
Jormakka, M., S. Tornroth, B. Byrne and S. Iwata (2002). "Molecular basis of proton motive force generation: structure of formate dehydrogenase-N." Science 295(5561): 18631868.

Keller, K. L., K. S. Bender and J. D. Wall (2009). "Development of a markerless genetic exchange system for Desulfovibrio vulgaris Hildenborough and its use in generating a strain with increased transformation efficiency." Appl Environ Microbiol 75(24): 7682-7691.

Keller, K. L., B. J. Rapp-Giles, E. S. Semkiw, I. Porat, S. D. Brown and J. D. Wall (2014). "New model for electron flow for sulfate reduction in Desulfovibrio alaskensis G20." Appl Environ Microbiol 80(3): 855-868.

Keller, K. L. and J. D. Wall (2011). "Genetics and molecular biology of the electron flow for sulfate respiration in Desulfovibrio." Front Microbiol 2: 135.

Keller, K. L., J. D. Wall and S. Chhabra (2011). "Methods for engineering sulfate reducing bacteria of the genus Desulfovibrio." Methods Enzymol 497: 503-517.

Kiefer, F., K. Arnold, M. Kunzli, L. Bordoli and T. Schwede (2009). "The SWISS-MODEL Repository and associated resources." Nucleic Acids Res 37: D387-D392.

Kikuchi, A., S. Y. Park, H. Miyatake, D. Sun, M. Sato, T. Yoshida and Y. Shiro (2001). "Crystal structure of rat biliverdin reductase." Nat Struct Biol 8(3): 221-225.

Kircher, M. and J. Kelso (2010). "High-throughput DNA sequencing--concepts and limitations." Bioessays 32(6): 524-536.

Kobayashi, K., S. Tachibana and M. Ishimoto (1969). "Intermediary formation of trithionate in sulfite reduction by a sulfate-reducing bacterium." J Biochem 65(1): 155-157.

Korte, H. L., S. R. Fels, G. A. Christensen, M. N. Price, J. V. Kuehl, G. M. Zane, A. M. Deutschbauer, A. P. Arkin and J. D. Wall (2014). "Genetic basis for nitrate resistance in Desulfovibrio strains." Front Microbiol 5(153): 1-12.

Kuehl, J. V., M. N. Price, J. Ray, K. M. Wetmore, Z. Esquivel, A. E. Kazakov, M. Nguyen, R. Kuehn, R. W. Davis, T. C. Hazen, A. P. Arkin and A. Deutschbauer (2014). "Functional genomics with a comprehensive library of transposon mutants for the sulfate-reducing bacterium Desulfovibrio alaskensis G20." MBio 5(3): e01041-01014.

Larsen, R. A., M. M. Wilson, A. M. Guss and W. W. Metcalf (2002). "Genetic analysis of pigment biosynthesis in Xanthobacter autotrophicus Py2 using a new, highly efficient transposon mutagenesis system that is functional in a wide variety of bacteria." Archives of Microbiology 178(3): 193-201.

Larsson, J. T., A. Rogstam and C. von Wachenfeldt (2005). "Coordinated patterns of cytochrome bd and lactate dehydrogenase expression in Bacillus subtilis." Microbiology 151(Pt 10): 3323-3335. 
Lee, S. D. Minchin and S. J. Busby (2012). "Activating transcription in bacteria." Annual Review of Microbiology 66: 125-152.

Lee, W., Z. Lewandowski, P. H. Nielsen and W. A. Hamilton (1995). "Role of SulfateReducing Bacteria in Corrosion of Mild-Steel - a Review." Biofouling 8(3): 165-194.

Li, M. Z. and S. J. Elledge (2007). "Harnessing homologous recombination in vitro to generate recombinant DNA via SLIC." Nature Methods 4(3): 251-256.

Lovley, D. R. (1993). "Anaerobes into heavy metal: Dissimilatory metal reduction in anoxic environments." Trends Ecol Evol 8(6): 213-217.

MacLean, B., D. M. Tomazela, N. Shulman, M. Chambers, G. L. Finney, B. Frewen, R. Kern, D. L. Tabb, D. C. Liebler and M. J. MacCoss (2010). "Skyline: an open source document editor for creating and analyzing targeted proteomics experiments." Bioinformatics 26(7): 966-968.

McLaughlin, K. J., C. M. Strain-Damerell, K. Xie, D. Brekasis, A. S. Soares, M. S. Paget and C. L. Kielkopf (2010). "Structural basis for NADH/NAD ${ }^{+}$redox sensing by a Rex family repressor." Mol Cell 38(4): 563-575.

McWilliam, H., W. Li, M. Uludag, S. Squizzato, Y. M. Park, N. Buso, A. P. Cowley and R. Lopez (2013). "Analysis Tool Web Services from the EMBL-EBI." Nucleic Acids Res 41(Web Server issue): W597-600.

Meyer, B., J. V. Kuehl, M. N. Price, J. Ray, A. M. Deutschbauer, A. P. Arkin and D. A. Stahl (2014). "The energy-conserving electron transfer system used by Desulfovibrio alaskensis strain G20 during pyruvate fermentation involves reduction of endogenously formed fumarate and cytoplasmic and membrane-bound complexes, Hdr-Flox and Rnf." Environmental Microbiology.

Murakami, K. S. and S. A. Darst (2003). "Bacterial RNA polymerases: the wholo story." Curr Opin Struct Biol 13(1): 31-39.

Muyzer, G. and A. J. Stams (2008). "The ecology and biotechnology of sulphate-reducing bacteria." Nat Rev Microbiol 6(6): 441-454.

Nakamura, A., A. Sosa, H. Komori, A. Kita and K. Miki (2007). "Crystal structure of TTHA1657 (AT-rich DNA-binding protein; p25) from Thermus thermophilus HB8 at 2.16 A resolution." Proteins 66(3): 755-759.

Noble, J. E. and M. J. Bailey (2009). "Quantitation of protein." Methods Enzymol 463: 7395.

Novichkov, P. S., T. S. Brettin, E. S. Novichkova, P. S. Dehal, A. P. Arkin, I. Dubchak and D. A. Rodionov (2012). "RegPrecise web services interface: programmatic access to the transcriptional regulatory interactions in bacteria reconstructed by comparative genomics." Nucleic Acids Res 40(W1): W604-W608. 
Novichkov, P. S., X. Li, J. V. Kuehl, A. M. Deutschbauer, A. P. Arkin, M. N. Price and D. A. Rodionov (2014). "Control of methionine metabolism by the SahR transcriptional regulator in Proteobacteria." Environmental Microbiology 16(1): 1-8.

Oliveira, T. F., C. Vonrhein, P. M. Matias, S. S. Venceslau, I. A. C. Pereira and M. Archer (2008). "The Crystal Structure of Desulfovibrio vulgaris Dissimilatory Sulfite Reductase Bound to DsrC Provides Novel Insights into the Mechanism of Sulfate Respiration." Journal of Biological Chemistry 283(49): 34141-34149.

Pagels, M., S. Fuchs, J. Pane-Farre, C. Kohler, L. Menschner, M. Hecker, P. J. McNamarra, M. C. Bauer, C. von Wachenfeldt, M. Liebeke, M. Lalk, G. Sander, C. von Eiff, R. A. Proctor and S. Engelmann (2010). "Redox sensing by a Rex-family repressor is involved in the regulation of anaerobic gene expression in Staphylococcus aureus." $\mathrm{Mol}$ Microbiol 76(5): 1142-1161.

Paget, M. S. and J. D. Helmann (2003). "The sigma ${ }^{70}$ family of sigma factors." Genome Biology 4(1): 203.

Parey, K., E. Warkentin, P. M. Kroneck and U. Ermler (2010). "Reaction cycle of the dissimilatory sulfite reductase from Archaeoglobus fulgidus." Biochemistry 49(41): 89128921.

Parks, J. M., A. Johs, M. Podar, R. Bridou, R. A. Hurt, Jr., S. D. Smith, S. J. Tomanicek, Y. Qian, S. D. Brown, C. C. Brandt, A. V. Palumbo, J. C. Smith, J. D. Wall, D. A. Elias and L. Liang (2013). "The genetic basis for bacterial mercury methylation." Science 339(6125): 1332-1335.

Parshina, S. N., J. Sipma, Y. Nakashimada, A. M. Henstra, H. Smidt, A. M. Lysenko, P. N. Lens, G. Lettinga and A. J. Stams (2005). "Desulfotomaculum carboxydivorans sp. nov., a novel sulfate-reducing bacterium capable of growth at 100\% CO." Int J Syst Evol Microbiol 55(Pt 5): 2159-2165.

Peck and J. LeGall (1994). Methods in Enzymology. San Diego, California, Academic Press, Inc.

Peck, H. D. (1959). "The ATP-Dependent Reduction of Sulfate with Hydrogen in Extracts of Desulfovibrio Desulfuricans." Proc Natl Acad Sci U S A 45(5): 701-708.

Peck, H. D. (1962). "The role of adenosine-5'-phosphosulfate in the reduction of sulfate to sulfite by Desulfovibrio desulfuricans." L Biol Chem 237: 198-203.

Pereira, I. A., A. R. Ramos, F. Grein, M. C. Marques, S. M. da Silva and S. S. Venceslau (2011). "A comparative genomic analysis of energy metabolism in sulfate reducing bacteria and archaea." Front Microbiol 2: 69.

Pereira, P. M., Q. He, F. M. Valente, A. V. Xavier, J. Zhou, I. A. Pereira and R. O. Louro (2008). "Energy metabolism in Desulfovibrio vulgaris Hildenborough: insights from transcriptome analysis." Antonie Van Leeuwenhoek 93(4): 347-362. 
Petersen, T. N., S. Brunak, G. von Heijne and H. Nielsen (2011). "SignalP 4.0: discriminating signal peptides from transmembrane regions." Nature Methods 8(10): 785-786.

Pfaffl, M. W. (2001). "A new mathematical model for relative quantification in real-time RTPCR." Nucleic Acids Res 29(9): e45.

Pfennig, N. and F. Widdel (1982). "The bacteria of the sulphur cycle." Philos Trans R Soc Lond B Biol Sci 298(1093): 433-441.

Price, M. N., A. M. Deutschbauer, J. V. Kuehl, H. C. Liu, H. E. Witkowska and A. P. Arkin (2011). "Evidence-Based Annotation of Transcripts and Proteins in the SulfateReducing Bacterium Desulfovibrio vulgaris Hildenborough." L Bacteriol 193(20): 57165727.

Ravcheev, D. A., X. Li, H. Latif, K. Zengler, S. A. Leyn, Y. D. Korostelev, A. E. Kazakov, P. S. Novichkov, A. L. Osterman and D. A. Rodionov (2012). "Transcriptional regulation of central carbon and energy metabolism in bacteria by redox-responsive repressor Rex." L Bacteriol 194(5): 1145-1157.

Ravot, G., B. Ollivier, M. Magot, B. Patel, J. Crolet, M. Fardeau and J. Garcia (1995). "Thiosulfate reduction, an important physiological feature shared by members of the order thermotogales." Appl Environ Microbiol 61(5): 2053-2055.

Redding-Johanson, A. M., T. S. Batth, R. Chan, R. Krupa, H. L. Szmidt, P. D. Adams, J. D. Keasling, T. S. Lee, A. Mukhopadhyay and C. J. Petzold (2011). "Targeted proteomics for metabolic pathway optimization: application to terpene production." Metab Eng 13(2): 194-203.

Reddy, S. G., G. Scapin and J. S. Blanchard (1996). "Interaction of pyridine nucleotide substrates with Escherichia coli dihydrodipicolinate reductase: thermodynamic and structural analysis of binary complexes." Biochemistry 35(41): 13294-13302.

Rodionov, D. A., I. Dubchak, A. Arkin, E. Alm and M. S. Gelfand (2004). "Reconstruction of regulatory and metabolic pathways in metal-reducing delta-proteobacteria." Genome Biology 5(11).

Rojo, F. (1999). "Repression of transcription initiation in bacteria." I Bacteriol 181(10): 29872991.

Rost, B. (1999). "Twilight zone of protein sequence alignments." Protein Eng 12(2): 85-94.

Roy, S., S. Garges and S. Adhya (1998). "Activation and repression of transcription by differential contact: two sides of a coin." L Biol Chem 273(23): 14059-14062.

Sass, A., H. Rutters, H. Cypionka and H. Sass (2002). "Desulfobulbus mediterraneus sp. nov., a sulfate-reducing bacterium growing on mono- and disaccharides." Archives of Microbiology 177(6): 468-474. 
Sawers, R. G., E. Zehelein and A. Bock (1988). "Two-dimensional gel electrophoretic analysis of Escherichia coli proteins: influence of various anaerobic growth conditions and the fnr gene product on cellular protein composition." Archives of Microbiology 149(3): 240-244.

Schau, M., Y. Chen and F. M. Hulett (2004). "Bacillus subtilis YdiH is a direct negative regulator of the cydABCD operon." L Bacteriol 186(14): 4585-4595.

Schiffer, A., K. Parey, E. Warkentin, K. Diederichs, H. Huber, K. O. Stetter, P. M. Kroneck and U. Ermler (2008). "Structure of the dissimilatory sulfite reductase from the hyperthermophilic archaeon Archaeoglobus fulgidus." L Mol Biol 379(5): 1063-1074.

Schlax, P. J., M. W. Capp and M. T. Record, Jr. (1995). "Inhibition of transcription initiation by lac repressor." L Mol Biol 245(4): 331-350.

Scotto-Lavino, E., G. Du and M. A. Frohman (2006). "5' end cDNA amplification using classic RACE." Nat Protoc 1(6): 2555-2562.

Shi, X. and D. L. Jarvis (2006). "A new rapid amplification of cDNA ends method for extremely guanine plus cytosine-rich genes." Anal Biochem 356(2): 222-228.

Sickmier, E. A., D. Brekasis, S. Paranawithana, J. B. Bonanno, M. S. Paget, S. K. Burley and C. L. Kielkopf (2005). "X-ray structure of a Rex-family repressor/NADH complex insights into the mechanism of redox sensing." Structure 13(1): 43-54.

Sim, M. S., D. T. Wang, G. M. Zane, J. D. Wall, T. Bosak and S. Ono (2013). "Fractionation of sulfur isotopes by Desulfovibrio vulgaris mutants lacking hydrogenases or type I tetraheme cytochrome c 3." Front Microbiol 4: 171.

Sporty, J. L., M. M. Kabir, K. W. Turteltaub, T. Ognibene, S. J. Lin and G. Bench (2008). "Single sample extraction protocol for the quantification of NAD and NADH redox states in Saccharomyces cerevisiae." L Sep Sci 31(18): 3202-3211.

Stoffels, L., M. Krehenbrink, B. C. Berks and G. Unden (2012). "Thiosulfate reduction in Salmonella enterica is driven by the proton motive force." L Bacteriol 194(2): 475-485.

Strain-Damerell, C. M. (2010). Functional analysis of Rex, a sensor of the NADH/NAD+ redox poise in Streptomyce coelicolor. $\mathrm{PhD}$, Sussex.

Summers, A. O. (1992). "Untwist and shout: a heavy metal-responsive transcriptional regulator." L Bacteriol 174(10): 3097-3101.

Sun, G., E. Sharkova, R. Chesnut, S. Birkey, M. F. Duggan, A. Sorokin, P. Pujic, S. D. Ehrlich and F. M. Hulett (1996). "Regulators of aerobic and anaerobic respiration in Bacillus subtilis." L Bacteriol 178(5): 1374-1385.

Thauer, R. K. (1989). Energy metabolism of sulfate reducing bacteria. Autotrophic Bacteria. Schlegal H.G. and B. B. Madison WI, USA, Science Tech Publishers: 397-413. 
Thauer, R. K., K. Jungermann and K. Decker (1977). "Energy Conservation in Chemotrophic Anaerobic Bacteria." Bacteriol. Rev. 41(1): 100-180.

Thauer, R. K., E. Stackebrandt and W. A. Hamilton (2007). "Energy metabolism and phylogenetic diversity of sulphate-reducing bacteria." Sulphate-Reducing Bacteria: Environmental and Engineered Systems: 1-37.

Thompson, J. D., D. G. Higgins and T. J. Gibson (1994). "CLUSTAL W: improving the sensitivity of progressive multiple sequence alignment through sequence weighting, position-specific gap penalties and weight matrix choice." Nucleic Acids Res 22(22): 4673-4680.

Titolo, S., K. Brault, J. Majewski, P. W. White and J. Archambault (2003). "Characterization of the minimal DNA binding domain of the human papillomavirus E1 helicase: Fluorescence anisotropy studies and characterization of a dimerization-defective mutant protein." L Virol 77(9): 5178-5191.

Turkarslan, S., E. J. Wurtmann, W. J. Wu, N. Jiang, J. C. Bare, K. Foley, D. J. Reiss, P. Novichkov and N. S. Baliga (2013). "Network portal: a database for storage, analysis and visualization of biological networks." Nucleic Acids Res.

Ullrich, T. C., M. Blaesse and R. Huber (2001). "Crystal structure of ATP sulfurylase from Sacharomyces cerevisiae, a key enzyme in sulfate activation." Embo Journal 20(3): 316329.

Valentin-Hansen, P., L. Sogaard-Andersen and H. Pedersen (1996). "A flexible partnership: the CytR anti-activator and the cAMP-CRP activator protein, comrades in transcription control." Mol Microbiol 20(3): 461-466.

Vandesompele, J., K. De Preter, F. Pattyn, B. Poppe, N. Van Roy, A. De Paepe and F. Speleman (2002). "Accurate normalization of real-time quantitative RT-PCR data by geometric averaging of multiple internal control genes." Genome Biology 3(7): RESEARCH0034.

Venceslau, S. S., Y. Stockdreher, C. Dahl and I. A. Pereira (2014). "The "bacterial heterodisulfide" DsrC is a key protein in dissimilatory sulfur metabolism." Biochim Biophys Acta 1837(7): 1148-1164.

Vesic, D. and C. J. Kristich (2013). "A Rex Family Transcriptional Repressor Influences H2O2 Accumulation by Enterococcus faecalis." L Bacteriol 195(8): 1815-1824.

Wall, J. D., A. P. Arkin, N. C. Balci and B. Rapp-Giles (2008). "Genetics and Genomics of Sulfate Respiration in Desulfovibrio." Microbial Sulfur Metabolism.

Wall, J. D., A. P. Arkin, N. C. Balci and B. Rapp-Giles (2008). Genetics and Genomics of

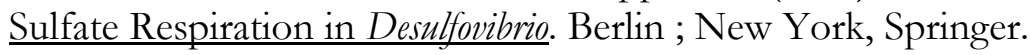

Wall, J. D. and L. R. Krumholz (2006). "Uranium reduction." Annual Review of Microbiology 60: 149-166. 
Wang, E., M. C. Bauer, A. Rogstam, S. Linse, D. T. Logan and C. von Wachenfeldt (2008). "Structure and functional properties of the Bacillus subtilis transcriptional repressor Rex." Mol Microbiol 69(2): 466-478.

Wang, E., T. P. Ikonen, M. Knaapila, D. Svergun, D. T. Logan and C. von Wachenfeldt (2011). "Small-angle X-ray scattering study of a Rex family repressor: conformational response to NADH and NAD ${ }^{+}$binding in solution." L Mol Biol 408(4): 670-683.

Wietzke, M. and H. Bahl (2012). "The redox-sensing protein Rex, a transcriptional regulator of solventogenesis in Clostridium acetobutylicum." Appl Microbiol Biotechnol 96(3): 749-761.

Williamson, D. H., P. Lund and H. A. Krebs (1967). "The redox state of free nicotinamideadenine dinucleotide in the cytoplasm and mitochondria of rat liver." Biochemical Journal 103(2): 514-527.

Wilson, K. P., L. M. Shewchuk, R. G. Brennan, A. J. Otsuka and B. W. Matthews (1992). "Escherichia coli biotin holoenzyme synthetase/bio repressor crystal structure delineates the biotin- and DNA-binding domains." Proc Natl Acad Sci U S A 89(19): 9257-9261.

Wing, H. J., S. M. Williams and S. J. Busby (1995). "Spacing requirements for transcription activation by Escherichia coli FNR protein." L Bacteriol 177(23): 6704-6710.

Xiong, J., D. M. Kurtz, Jr., J. Ai and J. Sanders-Loehr (2000). "A hemerythrin-like domain in a bacterial chemotaxis protein." Biochemistry 39(17): 5117-5125.

Zane, G. M., H. C. B. Yen and J. D. Wall (2010). "Effect of the Deletion of qmo $A B C$ and the Promoter-Distal Gene Encoding a Hypothetical Protein on Sulfate Reduction in Desulfovibrio vulgaris Hildenborough." Appl Environ Microbiol 76(16): 5500-5509.

Zhou, A., Y. I. Chen, G. M. Zane, Z. He, C. L. Hemme, M. P. Joachimiak, J. K. Baumohl, Q. He, M. W. Fields, A. P. Arkin, J. D. Wall, T. C. Hazen and J. Zhou (2012). "Functional characterization of Crp/Fnr-type global transcriptional regulators in Desulfovibrio vulgaris Hildenborough." Appl Environ Microbiol 78(4): 1168-1177.

Zhou, L., Q. E. Lim, G. Wan and H. P. Too (2010). "Normalization with genes encoding ribosomal proteins but not GAPDH provides an accurate quantification of gene expressions in neuronal differentiation of PC12 cells." Bmc Genomics 11: 75. 
Appendix 
Table A-1: Additional transcript data for Rex MED

\begin{tabular}{|c|c|c|c|c|c|c|}
\hline \multirow[b]{3}{*}{ Strain } & \multirow[b]{3}{*}{ Name } & \multirow[b]{3}{*}{ Media } & \multicolumn{4}{|c|}{ Mean $\mathrm{Cq}^{\mathrm{a}}$} \\
\hline & & & \multirow[b]{2}{*}{ sat } & \multirow[b]{2}{*}{ rex } & \multicolumn{2}{|c|}{ reference genes } \\
\hline & & & & & $r p l S$ & $r p m C$ \\
\hline JW710 & Parental & MOLS4 & $21.72 \pm 0.33$ & $22.60 \pm 0.02$ & $21.17 \pm 0.01$ & $23.14 \pm 0.01$ \\
\hline JW3311 & $\Delta r e x$ & MOLS4 & $19.50 \pm 0.31$ & $>35$ & $22.64 \pm 0.01$ & $24.62 \pm 0.00$ \\
\hline JW3311 (pMO3313) & Complement of rex & MOLS4 & $19.42 \pm 0.21$ & $19.32 \pm 0.01$ & $21.76 \pm 0.01$ & $23.42 \pm 0.02$ \\
\hline JW710 & Parental & MOYPyr & $20.88 \pm 0.26$ & $24.05 \pm 0.01$ & $23.56 \pm 0.05$ & $25.10 \pm 0.03$ \\
\hline JW3311 & $\Delta r e x$ & MOYPyr & $20.27 \pm 0.42$ & $>35$ & $23.78 \pm 0.01$ & $25.56 \pm 0.01$ \\
\hline JW3311 (pMO3313) & Complement of rex & MOYPyr & $21.71 \pm 0.13$ & $20.47 \pm 0.01$ & $24.32 \pm 0.00$ & $25.57 \pm 0.02$ \\
\hline
\end{tabular}

OD600 was monitored through growth for kinetic studies and samples were collected for analysis at early-exponential and generation time was determined. Approximately $100 \mathrm{ng}$ of TURBO ${ }^{\mathrm{TM}}$ DNase-treated RNA was converted to cDNA and 1 $\mu \mathrm{L}$ of cDNA ( $5 \mathrm{ng}$ of RNA) was used per qRT-PCR. Critical threshold (Cq) is presented for all genes tested, including two reference genes, $r p l S$ and $r p m C .{ }^{a}$ Critical threshold $(\mathrm{Cq})$ for specified gene, with technical error determined as standard error of the mean $(n=3)$. 
Table A-2: Sample collection and transcript analysis for sat promoter studies

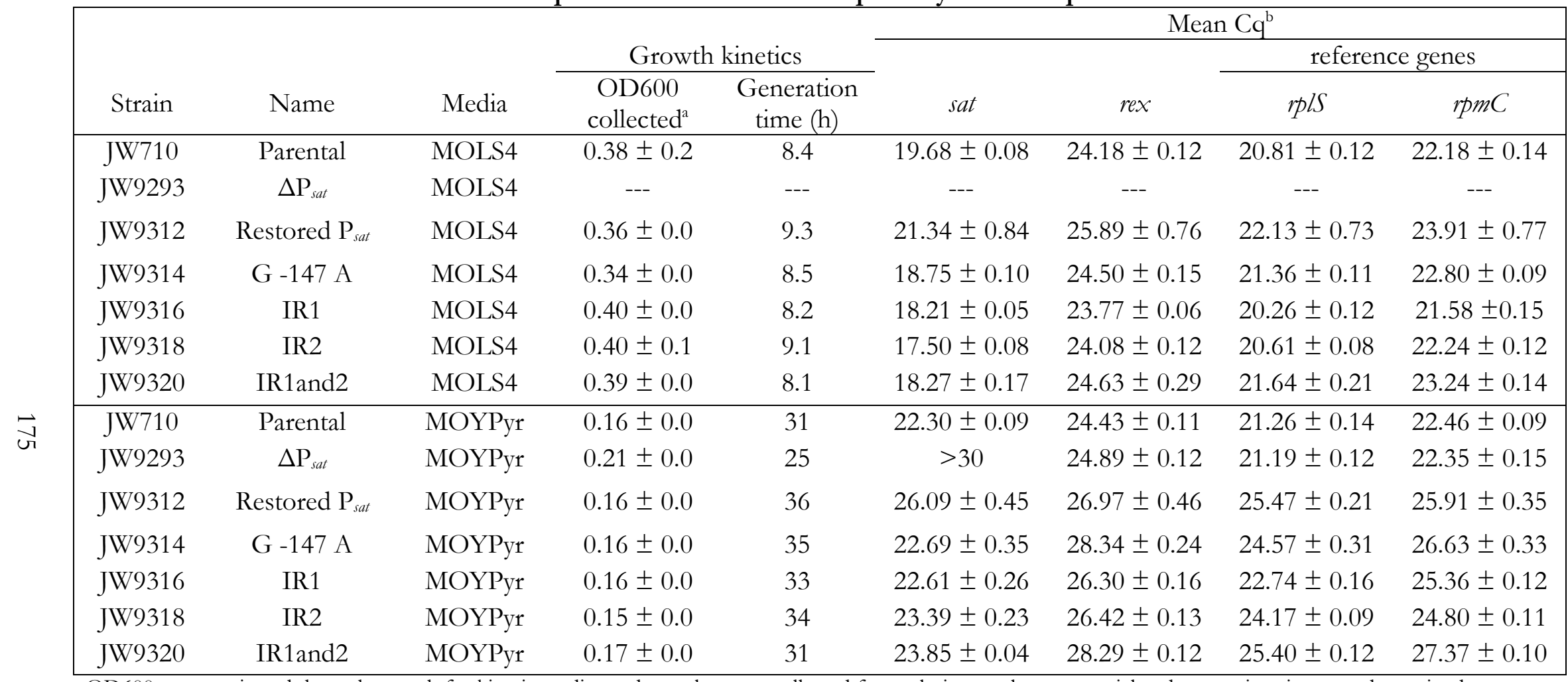

OD600 was monitored through growth for kinetic studies and samples were collected for analysis at early-exponential and generation time was determined. Approximately $100 \mathrm{ng}$ of TURBO ${ }^{\text {TM }}$ DNase treated RNA was converted to cDNA and $1 \mu \mathrm{L}$ of cDNA ( 5 ng of RNA) was used per qRT-PCR. Critical threshold $(\mathrm{Cq})$ is presented for all genes tested, including two reference genes, $r p l S$ and $r p m C$. a error determined as standard deviation $(\mathrm{n}=3)$. $\mathrm{b}$ Critical threshold $(\mathrm{Cq})$ for specified gene, with technical error determined as standard error of the mean $(n=3)$. 


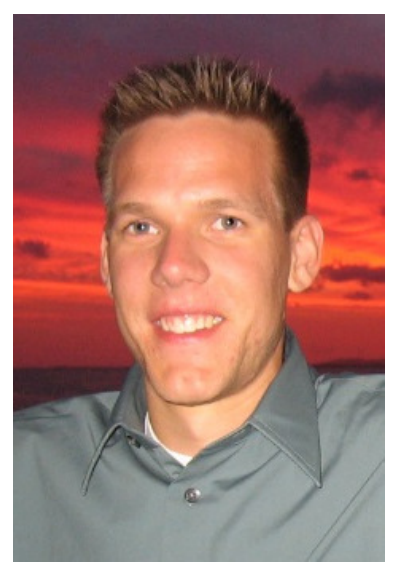

\section{Geoff A. Christensen, Ph.D.}

Geoff Christensen received his undergraduate degree from University of Illinois UrbanaChampaign (2008) and will receive his doctorate from University of Missouri-Columbia (2014), both in Biochemistry. He currently works with Dr. Judy Wall, where he is characterizing the redox repressor Rex and its role in regulating the metabolism of the sulfate reducer Desulfovibrio vulgaris Hildenborough. In pursuit of his degree he developed skills in molecular modeling, in culturing aerobes and anaerobes, in mutagenesis and purification and in analysis of RNA, DNA and protein. Additionally, he adapted qRT-PCR, RACE, and EMSA protocols for use in the Wall laboratory. Geoff has also become a valuable resource for several PIs starting to introduce transcript analysis techniques into their laboratories at Missouri. As a graduate student at Missouri, Geoff has been recognized for his efforts with many awards, including the National Institute of General Medical Sciences (NIGMS) Training Grant, Dr. Charles W. Gehrke Jr. Memorial Scholarship Fund and the Federation of European Microbiological Societies (FEMS) Young Scientist Grant. He has been active in the Biochemistry Graduate Student Organization and was the lead in many successful fundraisers that provided support for recruitment activities and development of collaborative interactions. 